Issued: September 1999

The Development and Application of

Reactive Transport Modeling Techniques to

Study Radionuclide Migration at

Yucca Mountain, NV

Hari Selvi Viswanathan 


\section{DISCLAIMER}

This report was prepared as an account of work sponsored by an agency of the United States Government. Neither the United States Government nor any agency thereof, nor any of their employees, make any warranty, express or implied, or assumes any legal liability or responsibility for the accuracy, completeness, or usefulness of any information, apparatus, product, or process disclosed, or represents that its use would not infringe privately owned rights. Reference herein to any specific commercial product, process, or service by trade name, trademark, manufacturer, or otherwise does not necessarily constitute or imply its endorsement, recommendation, or favoring by the United States Government or any agency thereof. The views and opinions of authors expressed herein do not necessarily state or reflect those of the United States Government or any agency thereof. 


\section{DISCLAIMER}

Portions of this document may be illegible in electronic image products. Images are produced from the best available original document. 


\section{ACKNOWLEDGEMENTS}

I would like to thank my friends and family for all of their support throughout the course of the work presented here. I would especially like to thank my parents for the encouragement that they have provided me during the span of this work and throughout my entire life.

I would also like to thank Professor Albert Valocchi for making this work possible. I have learned a great deal from Professor Valocchi about reactive transport modeling in porous media. $\mathrm{He}$ spent a great deal of time working on this research providing his valuable insight. I feel that he was an ideal advisor who allowed me to work independently but provided guidance when necessary. Dr. Bruce Robinson played an equally important role by sharing his expert knowledge on transport modeling of the Yucca Mountain site. Many of the ideas contained within this document have their roots within Professor Valocchi and Dr. Bruce Robinson.

Thanks to Gilles Bussod I was allowed to work on the Unsaturated Zone Transport Test. This provided me an unique opportunity to apply the models we developed to a field study. I would like to thank George Zyvoloski for allowing me to work under him at Los Alamos National Laboratory (LANL) when I was just a high school student. My credentials were rather mediocre at the time. Working with George at LANL proved to be very interesting and is largely responsible for why I have pursued a scientific career. He also made it possible to LANL to collaborate with the University of Illinois on this research. I would like to thank Professors Tom Johnson, Tim Ellsworth and Charlie Werth for serving on my dissertation committee. They provided me with thorough reviews of this manuscript. Appreciation is also expressed to Caroline Tebes-Stevens "who got me up to speed" on research when I first arrived at the University of Illinois. Finally I would like to thank Dr. Andy Wolfsberg and Dr. Peter Lichtner for helpful discussions during the course of this work.

Financial support for this project is gratefully acknowledged to the Earth and Environmental Sciences Division at Los Alamos National Laboratory. 



\section{TABLE OF CONTENTS}

LIST OF TABLES $\ldots \ldots \ldots \ldots \ldots \ldots \ldots \ldots \ldots \ldots \ldots \ldots \ldots \ldots$, ix

LIST OF FIGURES $\ldots \ldots \ldots \ldots \ldots \ldots \ldots \ldots \ldots \ldots \ldots \ldots \ldots \ldots \ldots \ldots$

1. INTRODUCTION $\ldots \ldots \ldots \ldots \ldots \ldots \ldots \ldots \ldots \ldots \ldots \ldots \ldots \ldots, 1$

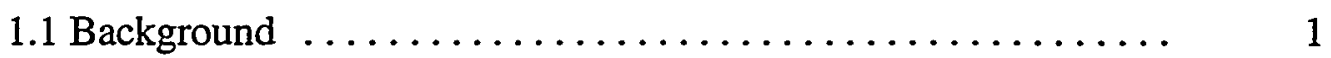

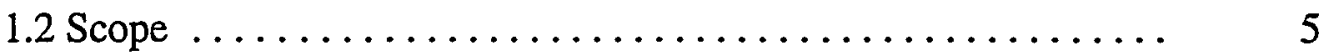

2. DEVELOPMENT OF THE CDR AND RST MODELS ....... 8

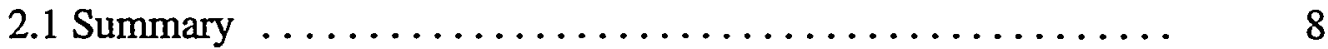

2.2 Development of the CDR Model ................. 9

2.2.1 Formulation of Physical/Chemical System .......... 9

2.2.2 Numerical Solution Techniques ................ 15

2.3 Development of the RST Model .................. 24

2.3.1 Computation of Streamlines ................. 25

2.3.2 Converting Streamlines into 1D Reactive Transport

Simulations ........................... 27

2.3.3 Example of the RST Method for a Nonreactive Tracer .... 31

3. VERIFICATION PROBLEMS FOR CDR $\ldots \ldots \ldots \ldots \ldots \ldots \ldots, 35$

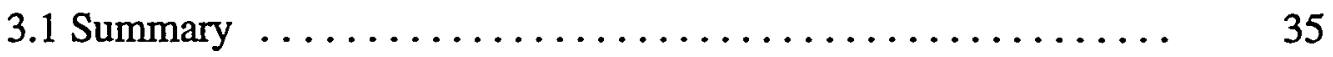

3.2 Example 1: Kinetic Formulation for Reaction Among Aqueous

Components ................................ 36

3.3 Example 2: CoNTA Transport Problem .............. 41

3.4 Example 3: ${ }^{237} \mathrm{~Np}$ Reactive Transport at Yucca Mountain ..... 47

4. VERIFICATION PROBLEMS FOR RST $\ldots \ldots \ldots \ldots \ldots \ldots \ldots, \quad 53$

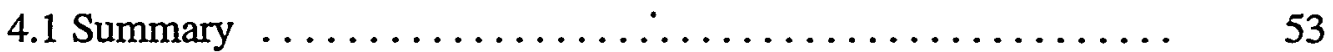

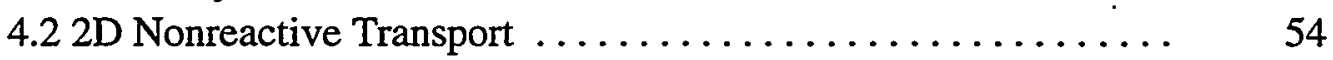

4.2.1 2D Vertical Layer Problem .................. 54

4.2.2 2D Bypass Problem ....................... 59

4.2.3 2D Random Field Problem .................... 64

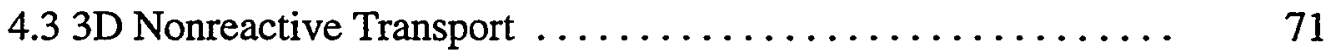

4.3.1 3D Vertical Layer Problem ................... 71

4.3.2 3D Bypass Problem ....................... 74

4.4.3 3D Random Field Problem .................. 76

4.4 2D Transport with Linear Retardation ............... 78

4.4.1 2D Bypass Problem ...................... 78

4.4.2 2D Problem with Random Flow and Retardation ....... 81

4.5 2D Multicomponent Transport Problem with Nonlinear Reactions $\quad 82$

4.5.1 Migration of ${ }^{237} \mathrm{~Np} \ldots \ldots \ldots \ldots \ldots \ldots \ldots \ldots . . . \ldots 2$

vii 
5. CALICO HILLS SIMULATIONS $\ldots \ldots \ldots \ldots \ldots \ldots \ldots \ldots \ldots, 87$

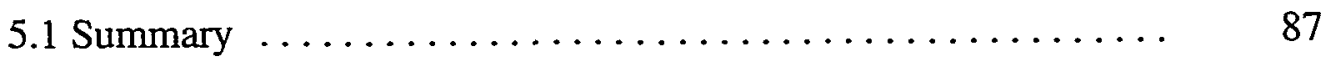

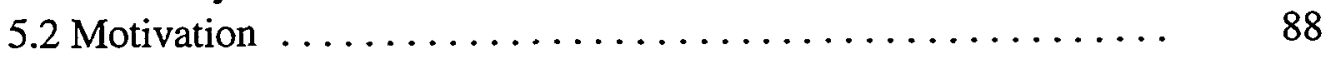

5.3 Implications of Zeolite Threshold Cutoff ............. 89

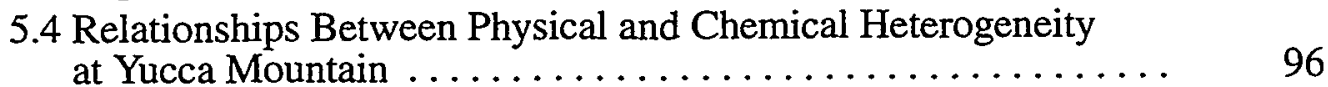

5.4.1 Distributing Zeolitic Abundance .............. 96

5.4.2 Correlation Between Permeability and Zeolitic Abundance $\quad 97$

5.4.3 Correlation Between Permeability and Relative Permeability 97

5.4.4 Correlation Between Permeability and Reactivity ....... 98

5.5 Simulations Utilizing CDR and RST ............... 99

5.5 .1 Summary $\ldots \ldots \ldots \ldots \ldots \ldots \ldots \ldots \ldots \ldots . . . \ldots \ldots$

5.5.2 Nonreactive Transport Simulations (Technecium) $\ldots \ldots . \quad 101$

5.5.3 Neptunium Transport Simulations ............... 107

6. THE UNSATURATED ZONE TRANSPORT TEST ......... 111

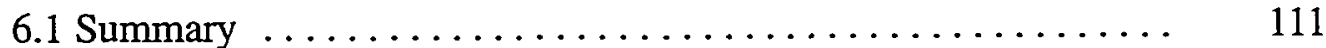

6.2 Background on Test $\ldots \ldots \ldots \ldots \ldots \ldots \ldots \ldots \ldots \ldots \ldots, 112$

6.3 Model Description ......................... 114

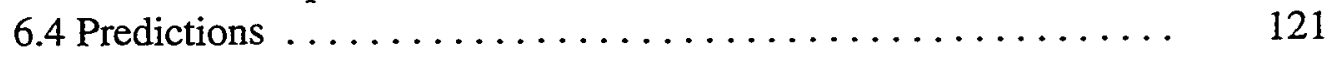

7. CONCLUSIONS $\ldots \ldots \ldots \ldots \ldots \ldots \ldots \ldots \ldots \ldots \ldots \ldots . . . \ldots \ldots$

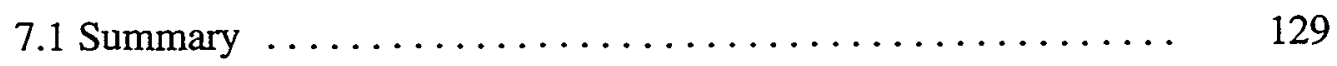

7.2 Selective Coupling ......................... 129

7.3 Reactive Streamtube Model ................... 134

7.4 The Unsaturated Zone Transport Test ............... 137

REFERENCES $\ldots \ldots \ldots \ldots \ldots \ldots \ldots \ldots \ldots \ldots \ldots \ldots \ldots \ldots \ldots$

VITA $\ldots \ldots \ldots \ldots \ldots \ldots \ldots \ldots \ldots \ldots \ldots \ldots \ldots \ldots \ldots \ldots \ldots, 145$ 


\section{LIST OF TABLES}

Table 2-1. Parameter values for RST Example ............ 31

Table 3-1. Computational efficiency for different levels of selective coupling: Example 1 ................. $\quad 39$

Table 3-2. Memory efficiency for a hypothetical 100,000 node simulation for different levels of selective coupling. ..... 41

Table 3-3. Components and concentrations of the background and injected fluid, CoNTA transport problem............. 43

Table 3-4. Equilibrium speciation reactions, CoNTA transport problem. ........................... 43

Table 3-5. Computational efficiency, CoNTA transport problem. ... 46

Table 3-6. Equilibrium speciation reactions, Neptunium transport problem. ............................. 50

Table 3-7. Computational efficiency, Neptunium transport problem. . 52

Table 4-1. Parameter values for $2 \mathrm{D}$ vertical layer problem ....... . $\quad 55$

Table 4-2. Comparison of CPU times between CDR and RST for $2 \mathrm{D}$ vertical layer cases .......................... 56

Table 4-3. Transverse mixing times $\ldots \ldots \ldots \ldots \ldots \ldots \ldots \ldots . .6$

Table 4-4. Parameters for two-dimensional flow bypass problem ... 59

Table 4-5. Test cases for two-dimensional flow bypass problem .... 60

Table 4-6. Comparison of CPU times between CDR and RST for

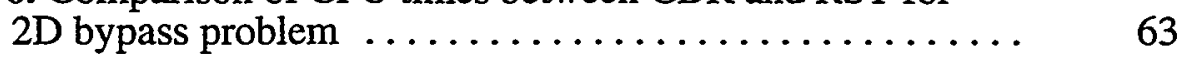

Table 4-7. Parameters for 2D random field problem .......... 64

Table 4-8. Comparison of CPU times between CDR and RST for $2 \mathrm{D}$ bypass problem..................... 67

Table 4-9. Comparison of CPU times between CDR and RST for 3D vertical layer problem $\ldots \ldots \ldots \ldots \ldots \ldots \ldots \ldots \ldots . \quad 73$

Table 4-10. Parameters for three-dimensional flow bypass problem . $\quad 74$

Table 4-11. Parameters for three-dimensional random field problem . 77

Table 4-12. Comparison of CPU times between CDR and RST for 3D vertical layer problem ..................... 77

Table 4-13. Comparison of CPU times between CDR and RST for 2D vertical layer problem with linear sorption .......... 81

Table 4-14. Parameters for $2 \mathrm{D}$ random field problems ......... 82

Table 4-15. Comparison of CPU times between CDR, PTRK, and RST for 2D vertical layer problem with linear sorption .... 82

Table 4-16. Parameters for ${ }^{237} \mathrm{~Np}$ transport problem ........... 84

Table 4-17. Aqueous component concentrations ............ 84

Table 4-18. Immobile component concentrations ........... 85

Table 4-19. Comparison of CPU times between CDR and RST for 2D vertical layer problem with linear sorption ......... 85

Table 5-1. Hydrologic and chemical properties of vitric and zeolitic

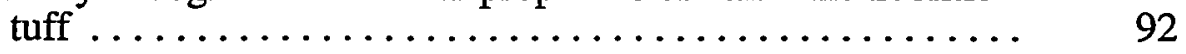

Table 5-2. Parameters used in the layer-bypass simulations ....... 94

Table 5-3. Parameters used in $64 \mathrm{~m}$ by $64 \mathrm{~m}$ Calico Hills simulations 101

Table 5-4. Comparison of CPU times between CDR and RST for low infiltration rate case with nonreactive tracer ........... 102 
Table 5-5. Comparison of CPU times between CDR and RST for high infiltration rate case with nonreactive tracer . . . . . 107

Table 5-6. Parameters for ${ }^{237} \mathrm{~Np}$ simulations .............. 108

Table 5-7. Comparison of CPU times between CDR and RST for ${ }^{237} \mathrm{~Np}$ transport for low infiltration rate case . . . . . . . 108

Table 5-8. Comparison of CPU times between CDR and RST for

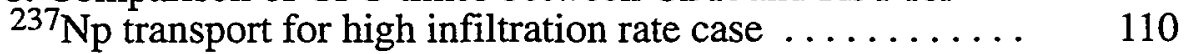

Table 6-1. Property sets for Phase 2 test . . . . . . . . . . 118

Table 6-2. Closest sampling point to the injection planes within each collection borehole $\ldots \ldots \ldots \ldots \ldots \ldots \ldots \ldots \ldots \ldots . \ldots \ldots$

Table 6-3. Comparison of CPU times between CDR and RST for simulation of UZTT $\ldots \ldots \ldots \ldots \ldots \ldots \ldots \ldots \ldots \ldots$ 


\section{LIST OF FIGURES}

Figure 2-1. An example of decomposing the domain into five streamtubes. There is no mixing between the streamtubes but there is complete mixing at the control plane. We obtain a flux-averaged breakthrough curve at the control

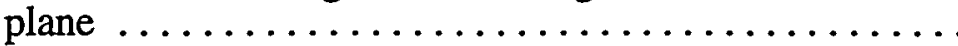

Figure 2-2. Schematic of the edge of cell velocities for a five-point block centered grid (Schafer-Perini and Wilson, 1991). .

Figure 2-3. Solution of the Pollock method providing position within the cell after time t. . ..................

Figure 2-4. Schematic for constructing one-dimensional reactive transport simulations from the information obtained from the Pollock (1988) method... . . . . . . . . . . .

Figure 2-5. Three dimensional test problem. . . . . . . . . . . 32

Figure 2-6. Plot of streamlines. Note that for this problem the 25 streamlines were released directly above the low

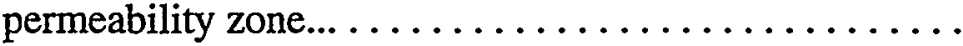

Figure 2-7. Comparison of one-dimensional transport simulations to analytical solutions.. . . . . . . . . . . . . .

Figure 3-1. Schematic of the one-dimensional model domain used for for Example 1.

Figure 3-2. Comparison of FEHM (full circles) and Tebes-Stevens and Valocchi (1998) solution for part A.. ...........

Figure 3-3. Stratigraphy and numerical grid for the Neptunium reactive transport problem (Example 3). a) dipping stratigraphy, including the location of zeolitic horizons; b) numerical grid of full model domain; c) close-up of grid at the edge of the potential repository.. ..........

Figure 3-4. Neptunium breakthrough mass flux at the water table. Curve a) equilibrium sorption reactions; b) slow reaction kinetics for sorption reactions (Case 1); c) rapid reaction kinetics for sorption reactions (Case 2); d) nonreactive tracer with the same repository release rate as a-c... ...

Figure 4-1. Two-dimensional flow with vertical layers. .........

Figure 4-2. Comparison of CDR, PTRK, and RST for transport of nonreactive transport through two layers with differing

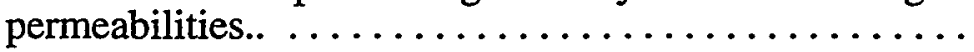

Figure 4-3. Vertical layer problem with different numbers of layers. Each layer is $5 \mathrm{~m}$ wide for 2 layer case, $2.5 \mathrm{~m}$ for 4 layer case, $1.25 \mathrm{~m}$ for 8 layer case, $0.625 \mathrm{~m}$ for 16 layer

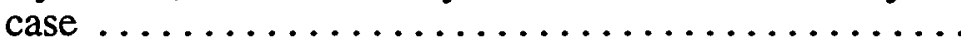

Figure 4-4. CDR model results that illustrate the effect of reducing the length scale for transverse dispersion. Note that the RST result is always equivalent to the 2 layer case since the model neglects transverse dispersion. ........

Figure 4-5. Two-dimensional flow bypass problem.. . . . . . . . . 
Figure 4-6. Twenty streamlines used by RST for two-dimensional

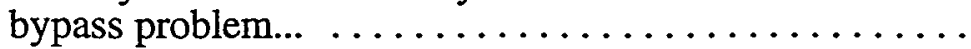

Figure 4-7. Flux averaged breakthrough curves comparing RST (case c), CDR with low transverse dispersion (case d), and PTRK with longitudinal dispersion but no transverse dispersion

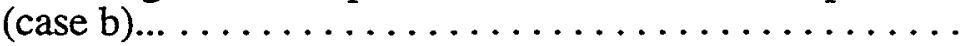

Figure 4-8. Flux averaged breakthrough curves comparing RST (case c), CDR with high transverse dispersion (case e), and PTRK with no dispersion (case a).. ................

Figure 4-9. Twenty streamlines used by RST to simulate nonreactive transport through a log normally distributed field of permeability.. . . . . . . . . . . . . . . .

Figure 4-10. Individual breakthrough curves from each streamline. A flux-weighted average of these breakthrough curves is used to obtain the RST solution. . ............

Figure 4-11. Flux averaged breakthrough curves comparing RST, CDR . and PTRK for nonreactive transport through a lognormally distributed permeability field... ..........

Figure 4-12. The effect of varying the number of streamlines. Flux averaged breakthrough curves of RST using 5, 10, and 20

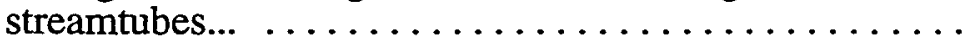

Figure 4-13. RST streamlines for different values of the $\ln (\mathrm{k})$ variance. Blue represents short travel times and red represents long travel times.. ...............

Figure 4-14. Comparison of RST and CDR for a $\ln (\mathrm{k})$ variance

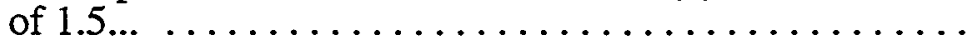

Figure 4-15. Comparison of $R S T$ and $C D R$ for a $\ln (k)$ variance

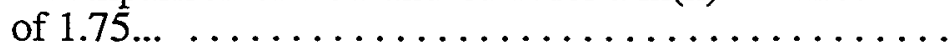

Figure 4-16. Comparison of RST and CDR for a $\ln (\mathrm{k})$ variance

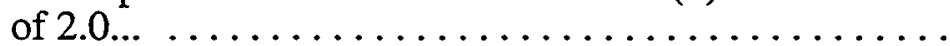

Figure 4-17. Three-dimensional flow problem with vertical layers...

Figure 4-18. Flux averaged breakthrough curves comparing the CDR, PTRK, and RST approaches for three-dimensional flow with vertical layers... . . . . . . . . . . . . . . 73

Figure 4-19. Three-dimensional flow bypass problem... ........ 74

Figure 4-20. Flux averaged breakthrough curves comparing the PTRK and RST methods for three-dimensional bypass problem..

Figure 4-21. Flux averaged breakthrough curves comparing the PTRK and RST methods for the three-dimensional random field problem

Figure 4-22. Flux averaged breakthrough curves comparing RST and CDR for two-dimensional flow bypass problem with linear retardation in the high permeability layer. Twenty streamlines are used for this simulation.. ...........

Figure 4-23. Flux averaged breakthrough curves comparing RST and CDR for two-dimensional flow bypass problem with linear retardation in the high permeability layer. Fifty streamlines are used for this simulation.. . . . . . . .

Figure 4-24. Two-dimensional random field problem with spatially correlated $\mathrm{K}_{\mathrm{d}}$. Twenty streamlines were used with RST. 
Figure 4-25. Comparison of the RST and CDR models for twodimensional ${ }^{237} \mathrm{~Np}$ transport with nonlinear reactions.. . .

Figure 5-1. Antler Ridge cross section (a) hydrologic zones including rock with greater than $10 \%$ zeolitic abundance, (b) hydrologic zones including rock with greater than $20 \%$ zeolitic

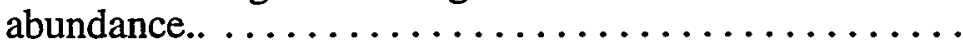

Figure 5-2. Two possible zeolitic geometries at the subgrid scale (10 $\mathrm{m}$ by $10 \mathrm{~m})$.

Figure 5-3. Comparison of the characteristic curves for zeolitic and

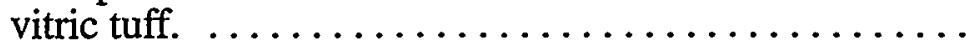

Figure 5-4. Velocity profile for the zeolitic lens case at three different infiltration rates.. . . . . . . . . . . . . . . . . .

Figure 5-5. Comparison of ${ }^{237} \mathrm{~Np}$ breakthrough curves for the layer

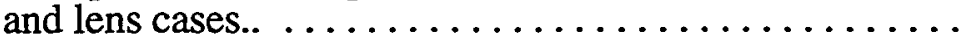

Figure 5-6. Data relating percent clinoptilolite to $\mathrm{K}_{\text {sat }}$ (from Flint, 1996).

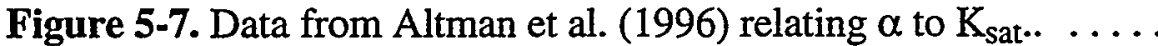

Figure 5-8. Schematic of $64 \mathrm{~m}$ by $64 \mathrm{~m}$ Calico Hills simulations. ...

Figure 5-9. (a) The saturation profile and (b) the velocity profile for the low saturation case. The infiltration rate across the block is approximately $0.1 \mathrm{~mm} / \mathrm{yr} . \ldots \ldots \ldots \ldots \ldots$

Figure 5-10. (a) The flow field is decomposed into eight equal flow streamtubes for the low saturation case. The solid lines represent the edges of the streamtubes and the dashed lines represent the center of the streamtubes, (b) the flux exiting the bottom boundary of the block.

Figure 5-11. (a) The saturation profile and (b) the velocity profile for the high saturation case. The infiltration rate across the block is approximately $4 \mathrm{~mm} / \mathrm{yr}$.

Figure 5-12. (a) The flow field is decomposed into eight equal flow streamtubes for the high saturation case. The solid lines represent the edges of the streamtubes and the dashed lines represent the center of the streamtubes, (b) the flux exiting the bottom boundary of the block. ...........

Figure 5-13. Comparison of the RST and CDR models for $0.1 \mathrm{~mm} / \mathrm{yr}$

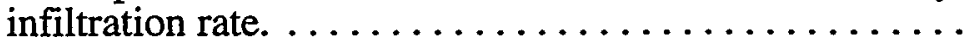

Figure 5-14. Comparison of the RST and CDR models for $4 \mathrm{~mm} / \mathrm{yr}$

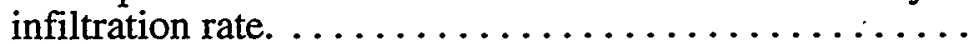

Figure 6-1. Schematic of the Busted Butte Phases. This schematic shows the locations of the different experiment phases and borehole locations. ..................... 112

Figure 6-2. View of Phase 2 injection and collection boreholes .... 114

Figure 6-3. Top view of finite element grid, injection and collection boreholes. ............................ 115

Figure 6-4. The finite element grid with injection boreholes as seen from the Test Alcove. The colored lines represent the location of collection boreholes which are perpendicular to the injection boreholes. . . . . . . . . . . . . . . . . . 
Figure 6-5. The finite element grid with collection boreholes as seen from the Main Adit. The horizontal colored lines represent the location of the injection boreholes which are perpendicular to the collection boreholes. The vertical colored line represents plunging collection boreholes. ...

Figure 6-6. Using ECM and Tptpv2 properties to predict Phase 1B. The nonreactive breakthrough of tracer $28 \mathrm{~cm}$ from the injection pad. (a) saturation breakthrough, (b) concentration breakthrough.

Figure 6-7. Comparison of the actual fluorescein cross section from phase $1 \mathrm{~B}$ mineback and numerical model. For further details see Bussod et al. (1998). . . . . . . . . . . . . . . . .

Figure 6-8. Saturation profile from 10 to $60 \%$ for a given realization: (a) background saturation, (b) saturation profile after 1 year

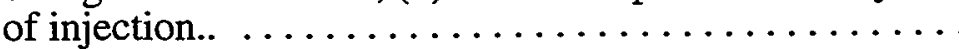

Figure 6-9. The concentration plume after 1 year of nonreactive tracer injection. The green isosurface represents a concentration of 0.5 . The red dots represent the sampling points along the collection boreholes.

Figure 6-10. Streamlines from the injection point to 1 year after injection.

Figure 6-11. Comparison of Phase 2 modeling predictions to actual measured breakthrough times. The lower bound for the predicted time is the $5 \%$ concentration breakthrough and the upper bound is the $50 \%$ concentration breakthrough. Note that the measured breakthrough are from $1 \%$ and $45 \%$ concentration breakthrough. ...............

Figure 6-12. Comparison of measured and predicted breakthrough times as a function of distance from the injection plane. . 


\title{
THE DEVELOPMENT AND APPLICATION OF REACTIVE TRANSPORT MODELING TECHNIQUES TO STUDY RADIONUCLIDE MIGRATION AT YUCCA MOUNTAIN, NV
}

\author{
Hari Selvi Viswanathan
}

Yucca Mountain, Nevada has been chosen as a possible site for the first high level radioactive waste repository in the United States. As part of the site investigation studies, we need to make scientifically rigorous estimations of radionuclide migration in the event of a repository breach. Performance assessment models used to make these estimations are computationally intensive. We have developed two reactive transport modeling techniques to simulate radionuclide transport at Yucca Mountain: 1) the selective coupling approach applied to the convection-dispersion-reaction (CDR) model and 2) a reactive streamtube approach (RST). These models were designed to capture the important processes that influence radionuclide migration while being computationally efficient. The conventional method of modeling reactive transport models is to solve a coupled set of multi-dimensional partial differential equations for the relevant chemical components in the system. We have developed an iterative solution technique, denoted the selective coupling method, that represents a versatile alternative to traditional uncoupled iterative techniques and the fully coupled global implicit method. We show that selective coupling results in computational and memory savings relative to these approaches. We develop RST as an alternative to the CDR method for solving large twoor three-dimensional reactive transport simulations for cases in which one is interested in predicting the flux across a specific control plane. In the RST method, the multidimensional problem is reduced to a series of one-dimensional transport simulations along streamlines. The key assumption with RST is that mixing at the control plane approximates the transverse dispersion between streamlines. We compare the CDR and RST approaches for several scenarios that are relevant to the Yucca Mountain Project. For example, we apply the CDR and RST approaches to model an ongoing field experiment called the Unsaturated Zone Transport Test. 


\section{INTRODUCTION}

\subsection{Background}

Yucca Mountain, Nevada, has been chosen as a possible site for the first high level radioactive waste repository in the United States. As part of the site investigation studies, we need to make scientifically rigorous estimations of radionuclide migration in the event of a repository breach. Numerous coupled processes will affect radionuclide migration, including multicomponent geochemical reactions, heat transport, vadose zone transport and fracture-matrix interactions. Also, the physical and geochemical properties of Yucca Mountain exhibit significant spatial variability that are impossible to characterize precisely. Due to these factors -- process complexity and spatial variability - there will be inherent error and uncertainty in any simulation model of transport at Yucca Mountain. In order to make a proper and accurate assessment of the performance of the potential repository, we need to develop transport simulation models that can capture the complexity of geochemical processes and the detailed spatial heterogeneity of the geological environment. In order to model the chemical and physical heterogeneities in the system, a three-dimensional grid with small grid spacing is often required. In addition, reactive transport simulations often involve the transport of numerous reacting chemical components which results in a system of coupled partial differential equations. Classical reactive modeling approaches are thus very computationally expensive to apply to this problem. In this thesis, we develop, verify and apply new modeling approaches that have enhanced computational efficiency.

Although simulation models for the transport of single chemical components have been widespread in the field of contaminant hydrogeology, multi-component geochemical models are a more recent development. Geochemical modeling takes many forms, including: 1) geochemical modeling without consideration of transport issues; 2) simulations of complex geochemical processes in one dimension; 3 ) contaminant plume migration in complex flow systems without consideration of the complexities of chemical reactions; and 4) models that combine complex 
reactive processes with detailed descriptions of groundwater flow. The recent trend in reactive transport modeling has been to include geochemical complexities such as biological reactions (Rittmann and VanBriesen, 1996), explicit characterization of root zone processes (Suarez and Simunek, 1996), multiphase reactions such as mineral dissolution/precipitation reactions and their feedback on the flow system (Lichtner, 1996), and the influence of heterogeneities (Gelhar, 1993). As the chemical aspects of these models become more complex, there has been considerable focus on numerical formulations that can solve the resulting equations efficiently. Steefel and MacQuarrie (1996) provide a summary of these methods and approaches.

In most reactive transport codes, the transport of chemical species is modeled using the convection-dispersion-reaction (CDR) equation. The method to incorporate the chemistry varies depending on the particular code. In general, the chemistry of the system can be modeled using a kinetic, equilibrium, or mixed kinetic-equilibrium formulation. The first codes that coupled complex chemistry with transport typically assumed that all chemical species in the system were in local equilibrium (e.g. Cederberg et al., 1985; Engesgaard and Kipp, 1992; Rubin, 1983; Yeh and Tripathi, 1989). Papers by Yeh and Tripathi (1989), Liu and Narasimhan (1989), and Lichtner (1996) review many of these models and discuss their approaches. Due to computational limitations and the virtually nonexistent data base of mineral-water reaction rates, the local equilibrium assumption was a necessity. Equilibrium approaches remain popular due to the accessibility of large geochemical databases and sophisticated software packages. Nevertheless, the current trend in reactive transport codes is to include kinetic formulations for certain types of reactions. Experimental evidence of kinetic limitations is shown in: Wood et al. (1990), Brusseau et al. (1989), Nicoud and Schweich (1989), van der Zee et al. (1989), Goltz and Roberts (1986), and Hutzler et al. (1986). In addition, there now exists a considerable body of knowledge of mineral kinetic rate constants (Steefel and Lasaga, 1994). Therefore, kinetic formulations are useful for these water-rock interactions since both kinetic and equilibrium behavior can be modeled with a kinetic formulation (Steefel and Lasaga, 1994). 
Various approaches have been taken to model combined kinetic and equilibrium transport systems. We will refer to these approaches as mixed equilibrium-kinetic formulation. The equations that arise from a mixed equilibrium-kinetic formulations can be cast so that the minimum number of independent unknowns are solved for while still incorporating the appropriate kinetic reactions. For example, Liu and Narasimhan (1989) include kinetic precipitation-dissolution in their otherwise equilibrium transport code. Steefel and Lasaga (1994) and Tebes-Stevens et al. (1998) assume aqueous phase reactions are in local equilibrium but include kinetic models for rock-water interactions. We will utilize a mixed equilibrium-kinetic formulation for the work presented in this thesis.

The coupled partial differential equations that arise in a reactive transport formulation are computationally expensive to solve. There are numerous solution techniques that have been presented in the literature that deal with the problem of how to couple the reaction and transport terms. These techniques can be broken down into three categories: operator splitting, the global implicit method, and sequential iteration approaches (SIA). Each technique has its advantages and disadvantages with respect to both accuracy and computational efficiency. Steefel and MacQuarrie (1996) compare these techniques in greater detail. In the global implicit method, the entire coupled system of chemical components is solved simultaneously. This approach is scientifically sound, since the reactions couple the components together. However, this method often leads to excessive memory requirements and CPU times. In SIA, each component is decoupled from the others resulting in a much smaller system of equations. However, SIA is an iterative scheme that can have trouble converging. Finally, operator splitting is a non-iterative two step process. Because the transport and reaction are formally separated, it is only necessary to solve for the nonreactive transport of one component at a time. However, operator splitting methods can incur mass balance errors if the kinetic reactions rates are fast or if large time steps are taken (Valocchi and Malmstead, 1992).

The selective coupling algorithm developed in this thesis utilizes a solution technique that is a hybridized version of the global implicit and sequential iteration methods presented in the 
literature. The method allows only the strongly coupled components' to be solved together. This approach can result in computational savings relative to the global implicit method by achieving a similar total SIA iteration count to thte global implict method while reducing the CPU time per iteration. More importantly, the memory requirements of the algorithm are controlled by the maximum number of coupled components rather than the number of components in the system. We will compare the performance of our method to the global implicit and sequential iteration methods. We will also apply our model to examples relevant to the Yucca Mountain Project.

Another approach to reduce the computational expense of the transport model is to make some simplifying assumptions to convert the large three-dimensional problem into onedimensional simulations along streamlines. The groundwater literature contains numerous streamtube models for various applications. Simmons et al.(1995) developed a method called the stochastic convective reactive (SCR) transport approach which recasts transport as the behavior of an ensemble of streamtubes with stochastically distributed travel times. Ginn et al. (1995) applied the SCR method to a reactive transport problem with a "canonical" one-dimensional streamtube. Yabusaki et al. (1998) present an advective reactive streamtube (ARS) transport technique that provides accurate solutions of nonlinear multicomponent reactive transport in nonuniform twodimensional velocity fields. They compare their solutions to a CDR model to evaluate the accuracy of the numerical formulation. Their technique includes mixed equilibrium and kinetic complexation and precipitation-dissolution reactions subject to the following assumptions: (1) transport is purely advective (i.e., no explicit diffusion or dispersion), and (2) chemistry is described by a canonical system of reactions that evolves with time and is unaffected by position in space. The models most relevant to our work are by Thiele et al. (1995), Blunt et al. (1996), and Batycky et al. (1996). They have developed streamtube models using the Pollock (1988) analytical method of calculating path lines to associate fluxes with particular streamlines. Their computationally efficient methods rely on decoupling the physics of the flow from the geologic heterogeneity. The influence of heterogeneity is captured with three-dimensional streamlines, whereas the physics of the flow is captured in the appropriate $1 \mathrm{D}$ solution and is mapped along 
each streamline. Their methods have been applied to model oil recovery and have compared well to conventional three-dimensional finite difference codes and field measurements. The major advantage of the method is the ability to model sharp fronts with a fast and accurate technique. We develop an approach similar to Batycky et al. (1996) but utilize streamlines to model multicomponent reactive transport. We want to simulate problems in which there is longitudinal dispersion along each streamline and chemical heterogeneities. To the author's knowledge other streamline/streamtube methods have not been developed for these cases.

We develop a reactive streamtube model (RST) as an alternative to solving large two- or three-dimensional reactive transport simulations with the CDR method. In three-dimensional simulations, the CDR method can result in either numerical dispersion errors or large CPU times due to the fine grid spacing and small time steps required to obtain an accurate solution. With RST, we are replacing one computationally intensive two- or three-dimensional CDR simulation with numerous computationally efficient one-dimensional CDR simulations. The key assumption of RST is the neglect of transverse dispersion between streamtubes. RST is a promising method for many of the problems that we will study because we are often interested in calculating the average flux across a specific control plane. For example, we may be interested in calculating the average flux leaving the unsaturated zone of Yucca Mountain. Although mixing between streamtubes is neglected in the model, we argue that the apparent dispersion caused by complete mixing at the control plane is much greater than transverse dispersion between streamtubes. Another reason the RST method is appropriate for the simulations of interest here is that we can usually assume a steady state flow field, therefore, the streamlines/streamtubes do not change as a function of time in these simulations.

\subsection{Scope}

In summary, we develop two reactive transport modeling techniques to simulate radionuclide transport at Yucca Mountain: 1) the selective coupling approach applied to the 
convection-dispersion-reaction (CDR) model and 2) a reactive streamtube approach (RST). The overall goal of the present study is to demonstrate numerical techniques that can be used to solve the mixed equilibrium-kinetic transport problem in large, complex two- and threedimensional domains; the techniques must be flexible, with wide applicability to a range of reactive transport systems. Therefore, both cpu time and memory usage must be handled efficiently. The techniques are developed in an existing finite element heat and mass transfer code called FEHM (Zyvoloski et al., 1997). FEHM is a three-dimensional heat and mass transport code simulator that can represent complex structures (e.g. faults and stratigraphy) and model non-linear processes such as vadose flow and heat flow. The Yucca Mountain Project Review, an external review committee appointed by the Department of Energy, selected three unsaturated zone codes for further development and application (Reeves et al., 1994): FEHM and TRACR3D of Los Alamos National Laboratory, and TOUGH of Lawrence Berkeley Laboratory. Our modifications to FEHM therefore make it possible to model multicomponent reactive transport for the large two- and threedimensional problem domains required for site scale performance assessment of Yucca Mountain.

We will study the migration of radionuclides through the Calico Hills unit at Yucca Mountain, a major barrier to radionuclide migration in the unsaturated zone. This unit is considered to be a major barrier since matrix flow (as opposed to fracture flow expected in many units) is expected in major portions of the unit and it contains reactive minerals which act to retard many radionuclides. An ongoing field experiment, the Unsaturated Zone Transport Test (UZTT), has been monitoring the migration of radionuclide analogs through the Calico Hills. We will apply the models we develop to the UZTT.

We have chosen to study the radionuclide ${ }^{237} \mathrm{~Np}$ because of its long half life $\left(2.14 \times 10^{6}\right.$ years), relatively high solubility under oxidizing conditions, low dose limits, and predicted low sorption. Performance assessment models have identified ${ }^{237} \mathrm{~Np}$ as a radionuclide of concern for 
the proposed repository (Wilson et al., 1994). ${ }^{237} \mathrm{~Np}$ participates in numerous chemical reactions that affect its migration through the Calico Hills. The dissolution of ${ }^{237} \mathrm{~Np}$ from the waste canister controls the amount of ${ }^{237} \mathrm{~Np}$ released into the aqueous phase. ${ }^{237} \mathrm{~Np}$ in the aqueous phase forms numerous aqueous complexes some of which sorb to the zeolitic tuffs that are present in the Calico Hills. Based on the geochemical conditions the zeolites can act to retard ${ }^{237} \mathrm{~Np}$ significantly (Viswanathan et al., 1998). The effect of zeolitization on ${ }^{237} \mathrm{~Np}$ migration is another process that we will investigate. Highly zeolitized areas will retard ${ }^{237} \mathrm{~Np}$ but these regions are also less permeable.

In chapter 2, we will develop the CDR and RST models. Chapter 3 contains verification problems for the CDR model. Chapter 4 contains verification problems for the RST model. These chapters also contain some benchmarking examples of the models to test their computationally efficiency. Chapter 5 focuses on radionuclide transport through the Calico Hills unit. We utilize both the CDR model and the RST model for these calculations. Finally, Chapter 6 contains a model of the UZTT. We utilize both the CDR and RST models to make predictions of when reactive and conservative tracers will breakthrough at specific locations in the test block. These predictions are compared to the most recent test results. The predictions utilize existing Yucca Mountain databases and provide a means of checking model abstractions and the current databases. 


\section{DEVELOPMENT OF THE CDR AND RST MODELS}

\subsection{Summary}

In this chapter we develop the CDR (Convection-Dispersion-Reaction) and RST (Reactive Streamtube) models. In section 2.2 , we present the governing equations and the numerical solution techniques for the CDR model. We develop an iterative solution technique for reactive transport problems called the selective coupling method, that represents a versatile alternative to traditional uncoupled iterative techniques and the fully coupled global implicit method. The chemical formulation developed allows a combination of equilibrium and kinetic reactions, and therefore is more versatile than a purely equilibrium-based formulation. However, this is a very challenging system for obtaining an efficient numerical solution. Traditional solution techniques sequentially compute the concentrations of aqueous components and evaluate reaction source/sink explicitly based upon concentrations from the previous iteration. The global implicit method is a complete Newton-Raphson approach where all aqueous components are solved simultaneously. This method is too computationally demanding in most realistic three-dimensional problems. The selective coupling method developed here allows only the strongly coupled components to be solved simultaneously. We also develop a method denoted coupled normalization to reduce the computational work and memory requirements for particular types of reactive transport problems. These approaches can result in computational savings relative to the global implicit method by achieving a similar iteration count while reducing the cpu time per iteration. More importantly, the memory requirements of the selective coupling technique are controlled by the maximum number of coupled components, rather than by the total number of components. For complex aqueous chemical systems and grids with a large number of nodes, memory efficiency is the characteristic that makes the selective coupling method particularly attractive relative to the global implicit method. In chapter 3 , we will 
compare the computational efficiency and accuracy of the selective coupling algorithm to other common approaches that are used to solve reactive transport problems.

In section 2.3 , we develop a reactive streamtube model (RST) as an alternative to solving large two- or three-dimensional reactive transport simulations with the CDR method. In threedimensional simulations, the CDR method results in either numerical dispersion errors or large CPU times due to the fine grid spacing and small time steps required to obtain an accurate solution. With RST, we are replacing one computationally intensive two- or three-dimensional CDR simulation with numerous computationally efficient one-dimensional CDR simulations. The key assumption of RST is the neglect of transverse dispersion between streamtubes. RST is a promising method for many of the problems because we are often mainly interested in calculating the average flux across a specific control plane. For example one of the main transport features of interest in performance asssessment is the average flux leaving the unsaturated zone of Yucca Mountain. Although mixing between streamtubes is neglected in the model, we argue that the anparent dispersion caused by complete mixing at the control plane can be much greater than transverse dispersion between streamtubes under many conditions. Another reason the RST method is appropriate for the simulations of interest here is that we can usually assume a steady state flow field and therefore, the streamlines/streamtubes do not change as a function of time in these simulations. In Chapter 4, we verify that in many cases, the RST method can obtain results similar to the CDR method, with significant computational savings.

\subsection{Development of the CDR Model}

\subsubsection{Formulation of Physical/Chemical System}

\subsubsection{Primary/Secondary Variables}

The reactive transport equations solved by FEHM are described in greater detail in Viswanathan et al.(1998), and thus we will only provide a brief summary in this section. FEHM uses aqueous, immobile and vapor components as the primary dependent variables (PDVs) in the 
reactive transport equations. In FEHM, the secondary dependent variables (SDVs) are uncomplexed aqueous component concentrations and aqueous complex concentrations. The PDVs and SDVs are related through the following relationship:

$$
C_{j}=c_{j}+\sum_{i=1}^{N_{x}} a_{i j} x_{i} \quad j=1, \ldots, N_{c}
$$

where $C_{j}$ is the total aqueous concentration of component $\mathrm{j}, c_{j}$ is the uncomplexed concentration of component $\mathrm{j}, x_{i}$ is the concentration of complex $\mathrm{i}, a_{i j}$ is the stoichiometric coefficient representing the number of moles of component $\mathrm{j}$ in complex $\mathrm{i}, N_{c}$ is the number of aqueous components and $N_{x}$ is the number of aqueous complexes.

For transient fluid flow, the generalized version of the reactive transport equation for an aqueous component is used

$$
\frac{\partial \bar{A}_{j}}{\partial t}=\nabla \bullet\left(\phi S_{l} D \nabla \rho_{l} C_{j}\right)-\nabla \bullet f_{c}+R_{j} \quad j=1, \ldots, N_{c}
$$

where $\bar{A}_{j}=\phi C_{j} S_{l} \rho_{l}$ is the solute mass stored per unit total volume of aquifer, $C_{j}$ (units of moles solute per mass water), $\phi$ is the porosity; $\rho_{l}$ is the liquid density, $f_{c}=\rho_{l} C_{j} u_{l}$ is the advective mass flux of solute, $D$ is the hydrodynamic dispersion tensor, $S_{l}$ is the saturation; $u_{l}$ is the Darcy velocity vector, and $R_{j}$ is the kinetic reaction source-sink term which transfers component $\mathrm{j}$ into and out of the liquid phase. To simplify the notation, we define the advection-dispersion operator:

$$
L_{l}(C)=\nabla \bullet f_{c}-\nabla \bullet\left(\phi S_{l} D \nabla \rho_{l} C_{j}\right)
$$

Equation 2-3, the convection-dispersion-reaction (CDR) equation, can then be rewritten as:

$$
\frac{\partial \bar{A}_{j}}{\partial t}+L_{l}\left(C_{j}\right)=R_{j} \quad j=1, \ldots, N_{c}
$$

The reactive transport equation for a vapor component takes on a similar form to Equation $2-4$ and is given by 


$$
\frac{\partial \bar{B}_{k}}{\partial t}+L_{v}\left(G_{k}\right)=R_{k} \quad k=1, \ldots, N_{v}
$$

where $\bar{B}_{k}=\phi G_{k} \rho_{k} S_{v}$ is the solute mass stored per unit total volume of aquifer, $G_{k}$ is the vapor concentration of component $\mathrm{k}$ (units of moles solute per mass vapor), $u_{v}$ is the vapor Darcy velocity vector and $N_{v}$ is the number of vapor components. The hydrodynamic dispersion tensor is assumed to reduce to longitudinal and transverse components (e.g. Bear, 1987).

Immobile components are not transported and are therefore treated using a simple mass balance given by

$$
\frac{\partial M_{m}}{\partial t}=R_{m} \quad m=1, \ldots, N_{i m}
$$

where $M_{m}$ is the mass solute per unit total volume of aquifer and $N_{i m}$ is the number of immobile components.

The reaction rate terms in Equations 2-4, 2-5, and 2-6 originate from the kinetic reactions in the system and may be nonlinear functions of the concentrations of the total aqueous components, uncomplexed aqueous components, aqueous complexes, immobile components and vapor components. FEHM is capable of modeling the following kinetic processes: linear adsorption, nonlinear Langmuir adsorption, ion/surface exchange, precipitation-dissolution and liquid-vapor interchange of solute. The specific kinetic models that are available are described in subsection 2.2.1.3. Equations 2-4, 2-5, and 2-6 result in a system of $\left(N_{c}+N_{i m}+N_{v}\right)$ nonlinear coupled partial differential equations (PDEs). Discretizing these equations results in a set of $\left(N_{c}+N_{i m}+N_{v}\right) N_{n}$ algebraic equations where $N_{n}$ is the number of spatial grid points. FEHM's method for solving this system of coupled PDEs will be discussed in subsection 2.2.2.

\subsubsection{Speciation Reactions}

Given all of the aqueous component concentrations, the uncomplexed aqueous component concentrations and aqueous complex concentrations can be calculated using chemical equilibrium 
theory. The chemical equilibrium calculations performed by FEHM are similar to the techniques used in batch geochemical software such as EQ3/6 (Wolery, 1992) or the Geochemist's Workbench (Bethke, 1996). A chemical reaction describing aqueous speciation can be written in the following general form

$$
\sum_{j=1}^{N_{c}} a_{i j} \hat{C}_{j} \Leftrightarrow \hat{X}_{i} \quad i=1, \ldots, N_{x}
$$

where $\hat{C}_{j}$ is the chemical formula for the aqueous component $j$, and $\hat{X}_{i}$ is the chemical formula for the aqueous complex i. FEHM assumes that all aqueous speciation reactions are at local equilibrium. The mass action expression for an aqueous component is given by

$$
K_{i}=x_{i} \prod_{j=1}^{N_{c}}\left(\gamma_{j} c_{j}\right)^{-a_{i j}}
$$

where $K_{i}$ is the equilibrium formation constant for complex $i$ and $\gamma_{j}$ is the activity coefficient for aqueous component $\mathrm{j}$. In the present study, we neglect ionic strength corrections (i.e. $\gamma_{j}=1$ ). Equations 2-7 and 2-8 can be combined to express the total aqueous concentration of component $j$ as a function of the uncomplexed component concentrations:

$$
C_{j}=c_{j}+\sum_{i=1}^{N_{x}} a_{i j} K_{i} \prod_{z=1}^{N_{c}} c_{z}^{a_{i z}} \quad j=1, \ldots, N_{c}
$$

Equation 2-9 results in a set of $N_{c}$ nonlinear algebraic equations to be solved for each uncomplexed component concentration $\left(c_{j}\right)$ given all of the total aqueous concentrations $\left(C_{j}\right)$.

\subsubsection{Kinetic Reaction Types}

Kinetic reactions modeled by FEHM cannot be described by a single reaction rate expression. Rate expressions are available to simulate sorption, precipitation/dissolution, dual Monod biodegradation, and a general reversible reaction. Additional kinetic rate expressions are available, however, we will only discuss the kinetic rate expressions that are used in the test cases 
section of the thesis. Note that the kinetic rate expressions do not necessarily conserve charge. In all of the reactive transport systems modeled in this paper, we do not attempt to model the complete set of chemical reactions, but attempt to model the chemical reactions that are relevant to the current study. Since all reactions are not included, a charge balance over the entire system cannot be calculated.

\section{Sorption Model}

The sorption models we use in the current study contain the same parameters that are measured in laboratory and field experiments for the various applications we have investigated. For this reason, we have chosen a linear kinetic sorption, and a kinetic ion-exchange model. The retardation of contaminants due to adsorption/desorption can be modeled with a linear kinetic sorption/desorption expression. The rate of adsorption/desorption of component $\mathrm{j}$ is given by:

$$
R_{j}=-\rho_{l} \phi S_{l} k_{m}\left(c_{j}-\frac{m_{j}}{K_{D}}\right)
$$

where $m_{j}$ is the immobile concentration, $k_{m}$ is the mass transfer coefficient, $K_{D}$ is the distribution coefficient. As $k_{m} \rightarrow \infty$, this expression reduces to the linear equilibrium isotherm (e.g. Jennings and Kirkner,1984; Valocchi, 1985).

\section{Biodegradation Model}

Biodegradation is an irreversible process in which bacteria oxidize an organic substrate to produce energy and biomass. In addition to biomass, the biodegradation process requires the presence of an electron acceptor (e.g. oxygen, nitrate, etc.) and nutrients (e.g. nitrogen and phosphorous). An example of a simplified biodegradation reaction is given by the following reaction (Tebes-Stevens et al., 1998):

$$
\text { Substrate }+ \text { Electron Acceptor }+ \text { Nutrients } \rightarrow \text { cells }+\mathrm{CO}_{2}+\mathrm{H}_{2} \mathrm{O}
$$

FEHM models the rate of biodegradation of a substrate with a multiplicative Monod model, which is given by (Tebes-Stevens et al., 1998): 


$$
R_{s}=-\rho_{l} \phi S_{l} q_{m} M_{b} \frac{S}{K_{s}+S} \frac{A}{K_{A}+A}
$$

where $\mathrm{S}$ is the aqueous concentration of substrate (a.k.a the electron donor), $\mathrm{A}$ is the aqueous concentration of the electron acceptor, and $\mathrm{M}_{\mathrm{b}}$ is the concentration of the immobile biomass. The parameter $q_{m}$ is the maximum specific rate of substrate utilization, which represents the maximum amount of substrate that can be consumed per unit mass of bacteria per unit time. The parameters $\mathrm{K}_{\mathrm{S}}$ and $\mathrm{K}_{\mathrm{A}}$ are the Monod half-maximum-rate concentrations for the electron donor and electron acceptor, respectively. The rate of microbial growth is given by the synthesis rate (which is proportional to the rate of substrate degradation) minus a first-order decay rate

$$
R_{\text {cells }}=-Y R_{s}-b\left(M_{b}-M_{b, \text { init }}\right)
$$

where $\mathrm{Y}$ is the microbial yield coefficient and $\mathrm{b}$ is the first-order microbial decay coefficient. In the above equation, the assumption is made that the background conditions are sufficient to sustain a microbial population of a given size; therefore, the biomass concentration is not allowed to fall below its initial background concentration, $M_{b \text {,init }}$.

\section{Precipitation/Dissolution Model}

A general reaction describing the precipitation/dissolution of a mineral $\mathrm{p}$ can be written in the following form:

$$
\hat{M}_{p} \Leftrightarrow \mu_{p 1} \hat{C}_{1}+\mu_{p 2} \hat{C}_{2}+\ldots+\mu_{p N_{c}} \hat{C}_{N_{c}}
$$

where $\hat{M}_{p}$ is the chemical formula for mineral $\mathrm{p}$, and $\mu_{p j}$ are stoichiometric coefficients. The equilibrium constant for this reaction is known as the solubility product. Since the activity of a pure solid is equal to one, the reaction quotient, $Q_{p}$, is defined as:

$$
Q_{p}=\prod_{j=1}^{N_{c}} c_{j}^{\mu_{p j}}
$$

At equilibrium, $Q_{p}$ is equal to the solubility product. The surface-controlled rate of precipitation/ 
dissolution of a mineral is given by:

$$
R_{j}=A_{s} \mu_{p j} k_{+}\left(1-\frac{Q_{p}}{K_{s p}}\right)
$$

where $A_{s}$ is reactive surface area of the mineral, $k_{+}$is the rate constant, and $K_{s p}$ is the solubility product (Lasaga, 1984). With this equation, a mineral will precipitate when it is supersaturated and dissolve when it is undersaturated. If there is no mineral present and it is undersaturated, $R_{j}=0$.

\subsubsection{Numerical Solution Techniques}

\subsubsection{Newton-Raphson Formulation}

The reactive transport equations given by Equations 2-4, 2-5 and 2-6 result in a set of nonlinear coupled PDEs. The numerical implementation of the transport step can be derived by rewriting Equations $2-4,2-5$ and $2-6$ in fully implicit time-discretized form:

$$
\begin{array}{ll}
\frac{C_{j}^{n+1}-C_{j}^{n}}{\Delta t}+L\left(C_{j}^{n+1}\right)=R_{j}^{n+1} & j=1, \ldots, N_{c} \\
\frac{G_{k}^{n+1}-G_{k}^{n}}{\Delta t}+L\left(G_{k}^{n+1}\right)=R_{k}^{n+1} & k=1, \ldots, N_{v} \\
\frac{M_{m}^{n+1}-M_{m}^{n}}{\Delta t}=R_{m}^{n+1} & m=1, \ldots, N_{i m}
\end{array}
$$

where $\mathbf{n}$ indicates the time step level. Reactive transport codes in the literature solve equations 217, 2-18 and 2-19 using either the global implicit, operator-splitting, or sequential iterative methods (Yeh and Tripathi, 1989; Steefel and MacQuarrie, 1996). The global implicit method solves the transport and reaction step simultaneously. On the other hand, operator splitting methods solve the transport and reaction steps in sequence without iteration. Finally, the sequential iterative approaches iterate between the transport and reaction steps until a converged global implicit solution is achieved. In this paper, we present a technique which is a hybridized version of the global implicit and sequential iteration methods. We have chosen an implicit method since many of the prob- 
lems we are interested in take place over long time scales. Implicit methods are often more efficient for such problems because of their ability to take larger time steps (Steefel and MacQuarrie, 1996). Problems for modeling advection dominated flows over shorter time scales would be better suited for operator-splitting methods (Steefel and MacQuarrie, 1996).

The reaction rate terms in equations $2-17,2-18$ and 2-19 can be estimated using a Taylor series expansion to linearize the reaction rate term

$$
\begin{aligned}
& R_{i}^{n+1, p+1}=R_{i}^{n+1, p}+\sum_{j=1}^{N_{c}}\left(\frac{\partial R_{i}}{\partial C_{j}}\right)^{n+1, p}\left(C_{j}^{n+1, p+1}-C_{j}^{n+1, p}\right)+ \\
& \sum_{k=1}^{N_{v}}\left(\frac{\partial R_{i}}{\partial G_{k}}\right)^{n+1, p}\left(G_{k}^{n+1, p+1}-G_{k}^{n+1, p}\right)+ \\
& \sum_{m=1}^{N_{i m}}\left(\frac{\partial R_{i}}{\partial M_{m}}\right)^{n+1, p}\left(M_{m}^{n+1, p+1}-M_{m}^{n+1, p}\right)+\ldots
\end{aligned}
$$

where $\mathrm{p}$ is the iteration level and i represents either an aqueous, vapor or immobile component.

The iterative methods in the literature differ in the degree to which terms in the summation of equation are included in the linearized expressions approximating equations 2-17, 2-18 and 219. The fully coupled approach, called the global implicit method (Steefel and Lasaga, 1994), uses all derivative terms in equation. This method, a full Newton-Raphson approximation, results in a large system of coupled linear equations stemming from the derivative terms of $R_{i}$ with respect to other aqueous, vapor, or immobile components. The approximate iterative approaches in the reactive transport literature often drop terms in the summations to decouple the linear equations into smaller equations sets that are solved sequentially. The sequential iterative approach described by Yeh and Tripathi (1989), Engesgaard and Kipp (1992) and Kinzelbach et al. (1991) ignore all derivative terms, using only $R_{i}^{n+1, p}$ in equation $2-20$. We will refer to this method as the SIA-0 method. 
The SIA-1 method estimates the reaction term as (Tebes-Stevens et al., 1998):

$$
R_{i}^{n+1, p+1}=R_{i}^{n+1, p}+\left(\frac{\partial R_{i}}{\partial C_{i}}\right)^{n+1, p}\left(C_{i}^{n+1, p+1}-C_{i}^{n+1, p}\right)
$$

The SIA-1 approach seeks to improve the estimate of $R_{i}^{n+1, p+1}$ by using the terms in the summations of equation 2-20 where $j=i, k=i$, and $m=i$. That is, for component $i$, the relationships with aqueous, vapor and immobile components other than $i$ are neglected, and the linear equations arising from the individual components are solved separately. We have found that the SIA-1 greatly improves convergence for large Damkohler number systems, when kinetics are fast compared to the transport time scale (Tebes-Stevens et al., 1998). SIA- 1 often outperforms SIA-0 because the $\partial R_{i} / \partial C_{i}$ term is often significant, whereas, $\partial R_{i} / \partial C_{j}, i \neq j$ terms are not. However, for certain reactions, the SIA-1 approach becomes quite inefficient because some of the cross-derivative terms that are neglected by SIA-1 are significant. This can occur when aqueous, vapor, or immobile components that are, in fact, coupled to one another are treated as though they were independent.

\subsubsection{Selective Coupling and Coupled Normalization}

Selective Coupling: In an extension of the SIA methods, we selectively include additional derivative terms that couple a subset of the components to one another to improve convergence. We call this method "Selective Coupling" to denote the flexibility of the implementation. Further details are presented in Robinson et al. (1999, in press). Since cross derivative terms, $\partial R_{i} / \partial C_{j}$, are now included in the calculation, sets of components must be solved simultaneously. We note, in passing, that in the extreme case in which only $\partial R_{i} / \partial C_{i}$ are calculated, selective coupling reduces to the SIA-1 method. At the other extreme, in which all components are coupled, selective coupling is equivalent to a global implicit solution scheme. The algorithm is best illustrated through specific examples. For this, we first consider the following system of kinetic reactions among aqueous components: 


$$
\begin{aligned}
& A \Leftrightarrow B ; R_{A}=-R_{B}=k_{f 1} C_{A}-k_{r 1} C_{B} \\
& B \Leftrightarrow C ; R_{B}=-R_{C}=k_{f 2} C_{B}-k_{r 2} C_{C} \\
& C \Leftrightarrow D ; R_{D}=-\mathrm{R}_{C}=k_{f 3} C_{C}-k_{r 3} C_{D}
\end{aligned}
$$

We start with the fully coupled formulation of the transport problem. The equation set resulting from the use of Newton's method to solve the nonlinear system of equations is represented in block matrix form below:

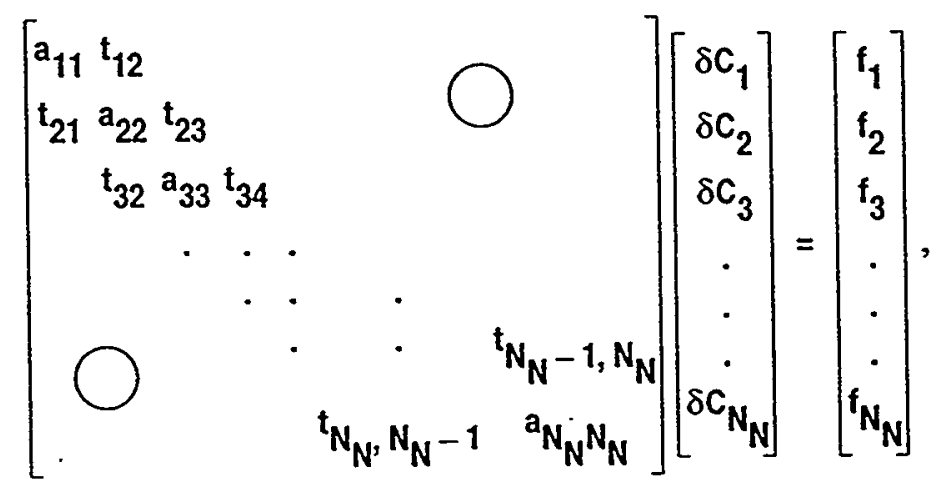

in which each element in the matrix is a $N_{d o f} \times N_{d o f}$ submatrix containing elements of the Jacobian matrix $\left(\partial f_{i} / \partial C_{j}\right)$, where $N_{d o f}$ is the number of "degrees of freedom," $\delta C$ is the vector of change in component concentration at node i $\left(\delta C_{A}, \delta C_{B}, \delta C_{C}\right.$ and $\left.\delta C_{D}\right), N_{N}$ is the number of spatial nodes, and the $f_{i}$ are the residual arrays which contain the advection, dispersion, accumulation, and reaction terms (of length $N_{d o f}$, in this case equal to four, the number of components in the example). The elements of the Jacobian matrix contain derivatives of the residual with respect to concentration. In 2-25 above, a tri-diagonal matrix resulting from a one-dimensional transport problem is shown for simplicity, but is not a restriction of the method. For our example reactive transport system, the submatrix $\mathbf{a}_{\mathrm{qq}}$ and submatrix $\mathbf{t}_{\mathrm{q}, \mathrm{q}+1}$ at a node $\mathrm{q}$ are given by: 


$$
\begin{aligned}
& a_{q q}=\left[\begin{array}{cccc}
\frac{\partial f_{A}}{\partial C_{A}} & \frac{\partial f_{A}}{\partial C_{B}} & 0 & 0 \\
\frac{\partial f_{B}}{\partial C_{A}} & \frac{\partial f_{B}}{\partial C_{B}} & \frac{\partial f_{B}}{\partial C_{C}} & 0 \\
0 & \frac{\partial f_{C}}{\partial C_{B}} \frac{\partial f_{C}}{\partial C_{C}} & \frac{\partial f_{C}}{\partial C_{D}} \\
0 & 0 & \frac{\partial f_{D}}{\partial C_{C}} \frac{\partial f_{D}}{\partial C_{D}}
\end{array}\right]
\end{aligned}
$$

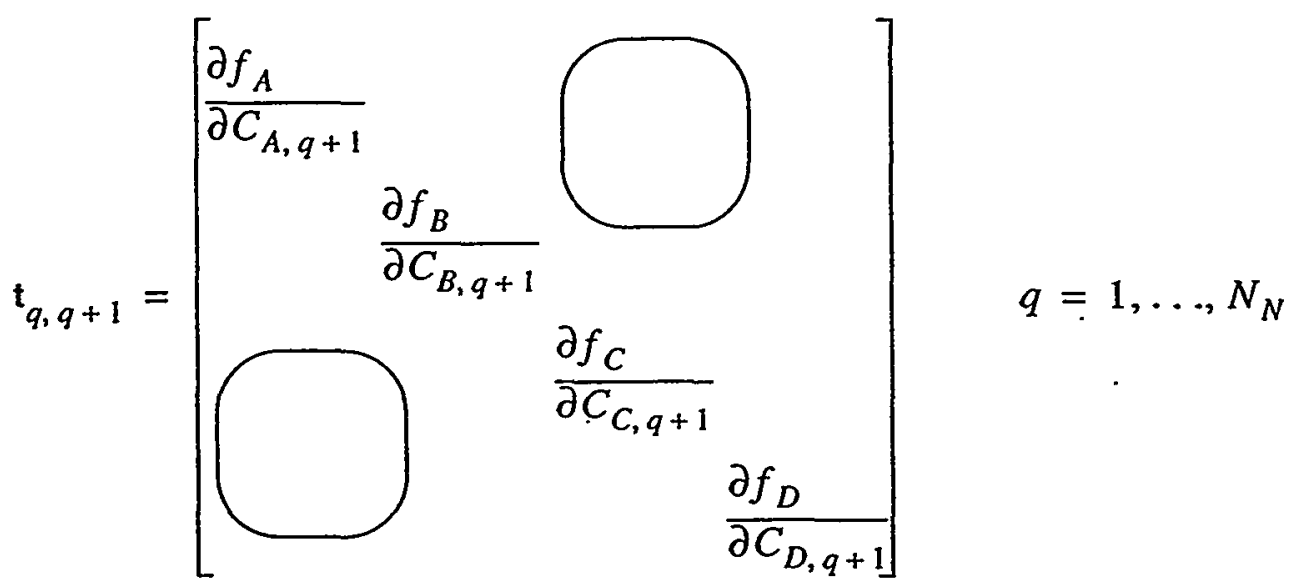

In general, the structure of submatrix $\mathbf{a}$ is defined by the reaction system. Note that the zeros in $\mathbf{a}_{\mathrm{qq}}$ are because not every component appears in each reaction. Now suppose that reaction (223 ) is slow compared to the other two reactions. In the submatrix $\mathbf{a}$, the terms $\partial f_{B} / \partial C_{C}$ and $\partial f_{C} / \partial C_{B}$ would be small compared to the other elements of $\mathbf{a}$, and $\mathbf{a}$ can be approximately represented as: 


$$
a=\left[\begin{array}{cccc}
\frac{\partial f_{A}}{\partial C_{A}} \frac{\partial f_{A}}{\partial C_{B}} & 0 & 0 \\
\frac{\partial f_{B}}{\partial C_{A}} \frac{\partial f_{B}}{\partial C_{B}} & 0 & 0 \\
0 & 0 & \frac{\partial f_{C}}{\partial C_{C}} \frac{\partial f_{C}}{\partial C_{D}} \\
0 & 0 & \frac{\partial f_{D}}{\partial C_{C}} \frac{\partial f_{D}}{\partial C_{D}}
\end{array}\right]
$$

This system can then be solved in two steps, with the $A$ and $B$ equations solved simultaneously, followed by the $C$ and $D$ equations solved in a second step. A reactive transport computer code that employs this method allows the user to "selectively couple" the components in the transport iteration. The decomposition of a four-degree-of-freedom problem into two 2-degreeof-freedom problems results in memory efficiencies and computational savings per iteration, and therefore is a desirable alternative to a full global implicit solution for some applications. Note that when all off-diagonal terms in the submatrix a are omitted, each of the four components can be solved iteratively and sequentially, i.e. the SIA-1 method is obtained.

When more than one component is solved simultaneously, an equation solver that handles block matrices is required. The linear equation solver in FEHM, developed primarily for the solution of coupled fluid flow and heat transport, makes use of well-tested numerical techniques that take advantage of the block structure of the coupled equations for pressure, temperature, and fluid saturation (Behie et al., 1985; Zyvoloski, 1983). Here, we use the same solver technologies for the transport solution step. In essence, for $N_{\text {dof }}$ unknowns per grid point, the same operations on the overall matrix of a single-unknown solution are carried out, but multiplications of individual matrix elements now become matrix multiplications involving the $N_{d o f} \times N_{\text {dof }}$ submatrices, and divisions are carried out as multiplications by the inverse of the submatrix. Since such operations become memory- and cpu-intensive for large problems, it is important to employ efficient numerical techniques. FEHM uses numerical methods suitable for the nonsymmetric matrices that 
arise from the finite-element solution of reactive transport equations on unstructured numerical grids. The solver software uses incomplete factorization with variable fill-in level (Zyvoloski, 1986) as a preconditioner, and a generalized minimum residual (GMRES) acceleration technique (Saad and Schultz, 1986) for the iterative solution. Details of this method as applied to heat and mass transport problems may be found in Zyvoloski et al. (1997). In a typical reactive transport solution with FEHM, the heat and mass transfer solution is also being performed, so the initial bookkeeping associated with the method is already being carried out, and the memory allocated for the solution is shared between the heat and mass solution and the transport solution.

There are definite trade-offs in computational efficiency and memory utilization between the SLA techniques and methods involving coupling of the transport equations of individual components. Coupling requires more time per iteration and more memory than typical SIA (i.e. SIA-0) methods. However, components strongly coupled by reaction may not converge using SIA methods unless small time steps are used. Since flexibility is required in a general purpose code, the transport iteration in FEHM was developed with the Selective Coupling provision to solve the component concentrations in groups of one or more at a time, so that only those components that need to be coupled are solved simultaneously. It is necessary to solve a set of equations for each component present in the system, but the order of solution and the nature of the coupling are set by the user at run-time. This allows the user, on the basis of information about the reactive transport system, to couple only those components that are required for efficient solution of the system of equations. Selective Coupling of components linked to each other through kinetic chemical reactions allows a given problem to be solved in the fastest, yet most memory efficient, manner possible (Viswanathan, 1996).

Coupled Normalization: When residual equations are solved simultaneously, it is advisable to normalize them so that they are solved to the same degree of numerical precision. We now present a method called "Coupled Normalization" for accomplishing this in a manner that in some cases has the added benefit of effectively reducing the number of degrees of freedom of the solution. Again, we will make use of an example to illustrate the method, in this case a kinetic ion exchange reaction 
of the form

$$
\begin{aligned}
& A+B-X \Leftrightarrow B+A-X \\
& R_{A}=k_{f}[A][B-X]-k_{r}[B][A-X]
\end{aligned}
$$

This example consists of two aqueous components $(A, B)$, which in general can undergo aqueous speciation reactions (though not in this example) and two immobile components (A-X, B-X). As in the previous example, $N_{\text {dof }}$ is four because we will solve for these four components simultaneously. Furthermore, the block matrix equation set in 2-25 also applies here. The submatrices a and $\mathbf{t}$ are given by:

$$
\begin{aligned}
& a_{q q}=\left[\begin{array}{llll}
\frac{\partial f_{A}}{\partial C_{A}} & \frac{\partial f_{A}}{\partial C_{B}} & \frac{\partial f_{A}}{\partial M_{A-X}} & \frac{\partial f_{A}}{\partial M_{B-X}} \\
\frac{\partial f_{B}}{\partial C_{A}} & \frac{\partial f_{B}}{\partial C_{B}} & \frac{\partial f_{B}}{\partial M_{A-X}} & \frac{\partial f_{B}}{\partial M_{B-X}} \\
\frac{\partial f_{A-X}}{\partial C_{A}} & \frac{\partial f_{A-X}}{\partial C_{B}} & \frac{\partial f_{A-X}}{\partial M_{A-X}} & \frac{\partial f_{A-X}}{\partial M_{B-X}} \\
\frac{\partial f_{B-X}}{\partial C_{A}} & \frac{\partial f_{B-X}}{\partial C_{B}} & \frac{\partial f_{B-X}}{\partial M_{A-X}} & \frac{\partial f_{B-X}}{\partial M_{B-X}}
\end{array}\right] \\
& \mathbf{t}_{q, q+1}=\left[\begin{array}{cccc}
\frac{\partial f_{A}}{\partial C_{A, q+1}} & 0 & 0 & 0 \\
0 & \frac{\partial f_{B}}{\partial C_{B, q+1}} & 0 & 0 \\
0 & 0 & 0 & 0 \\
0 & 0 & 0 & 0
\end{array}\right]
\end{aligned}
$$

The immobile components are not present in the $\mathbf{t}$ submatrices since there are no transport terms associated with them. Before solving the linear equation set, we apply the Coupled Normalization step, which consists of multiplying both sides of the equation set at each node by 
the inverse of $\mathbf{a}$, which of course transforms each submatrix $\mathbf{a}$ into the identity matrix. This operation scales the diagonal term of each equation to the same value (unity), thereby normalizing the equation set to ensure that when a convergence criterion, such as the $L_{2}$ norm, is employed, each equation is solved to the same level of accuracy on a normalized basis. Coupled normalization serves another important function for systems with immobile components, as evidenced by the structure of the transport submatrix $\mathbf{t}^{\prime}\left(=\mathbf{t a}^{-\mathbf{1}}\right)$

$$
\mathbf{t}^{\prime}=\left[\begin{array}{llll}
X & X & 0 & 0 \\
X & X & 0 & 0 \\
X & X & 0 & 0 \\
X & X & 0 & 0
\end{array}\right]
$$

where the $X$ denotes a non-zero term. After coupled normalization, the mobile component equations no longer contain terms involving the immobile component unknowns in either the transformed submatrix a or the $t^{\prime}$ submatrix. Thus, the first two equations can now be solved as a coupled two-by-two equation set for the concentration changes of the mobile species, after which the immobile component unknowns are then solved for individually by simple back-substitution. Coupled normalization in effect folds the cross-derivative information from the immobile component equations into the equations for the mobile components, so that only the two mobile component unknowns need be solved simultaneously. This method effectively reduces the number of degrees-of-freedom in the transport solution by the number of immobile components in the system of equations (two in this example), thereby reducing the memory utilization and computational burden of the solution.

In Chapter 3 we present examples that test the selective coupling and coupled normalization techniques and illustrate their power and flexibility for solving reactive transport problems. 


\subsection{Development of the RST Model}

In this section, we develop a reactive streamtube model (RST) as an alternative to solving large two- or three-dimensional reactive transport simulations with the CDR method. In threedimensional simulations, the CDR method results in either numerical dispersion errors or large CPU times due to the fine grid spacing and small time steps required to obtain an accurate solution. With RST, we are replacing one computationally intensive two- or three-dimensional CDR simulation with numerous computationally efficient one-dimensional CDR simulations.The key assumption of RST is the neglect of transverse dispersion between streamtubes. RST is a promising method for many of the problems that we will study because we are often mainly interested in calculating the average flux across a specific control plane. For example, we may be interested in calculating the average flux leaving the unsaturated zone of Yucca Mountain. Although mixing between streamtubes is neglected in the model, we argue that the apparent dispersion caused by complete mixing at the control plane is much greater than transverse dispersion between streamtubes (see Figure 2-1). We will examine the effects of this assumption in Chapter 4. Another reason the RST method is appropriate for the simulations of interest here is that we can usually assume a steady state flow field and therefore, the streamlines/streamtubes do not change as a function of time in these simulations.

First, we develop the methodology of calculating streamlines and groundwater travel times along each streamtube from two- or three-dimensional flow fields. Next, each streamtube is converted into a one-dimensional reactive transport simulation that is conducted using the CDR model described in section 2.2.2. A weighted average of the fluxes from each one-dimensional simulation is used to calculate the breakthrough at the control plane of interest. The methodology we develop will be general enough to allow us to use other reactive transport codes if necessary once the one-dimensional streamtubes are extracted from the FEHM flow field. However, we will use the FEHM CDR model to conduct the one-dimensional reactive transport simulations. This 


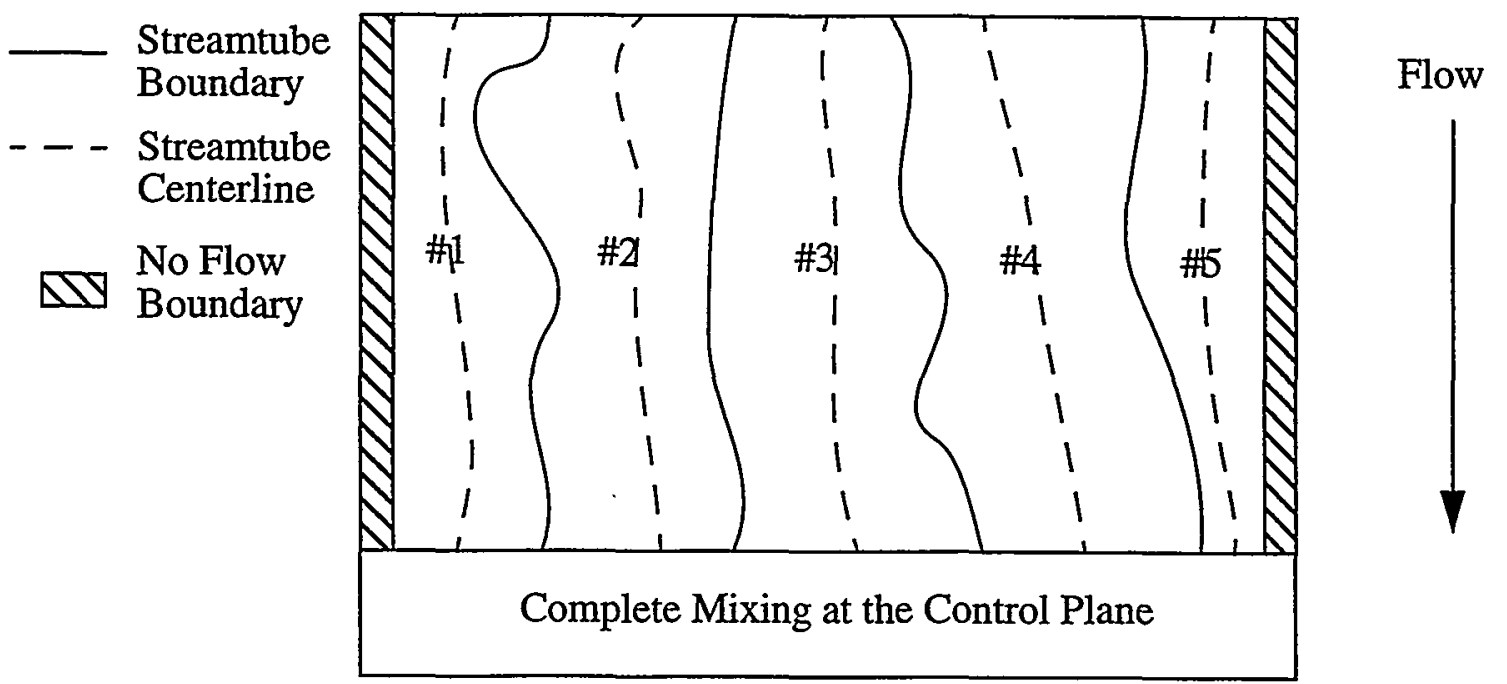

Figure 2-1. An example of decomposing the domain into five streamtubes. There is no mixing between the streamtubes but there is complete mixing at the control plane. We obtain a flux-averaged breakthrough curve at the control plane.

allows us to take advantage of the computational efficienciecs of the selective coupling and coupled normalization techniques we have developed.

\subsubsection{Computation of Streamlines}

The first step in developing the RST approach is to obtain streamlines from the flow field. Numerous methods exist to obtain streamlines. We have chosen a "semi-analytical" particle tracking method outlined by Pollock (1988) since it was developed for block centered models such as FEHM. The method is described as semi-analytical since the overall path of the particle is the summation of a series of analytically determined path lines for individual grid cells. The algorithm is simple and accounts for no flow boundaries implicitly. Finally, the method is completely general and can be applied to transient flow problems although the computational efficiency of the method is diminished since particle locations must be computed at the end of each flow time step. We will primarily consider steady state flow fields.

The Pollock (1988) method is ideal for use with FEHM since the FEHM flow solution provides the mass fluxes of each phase across the element faces. Node based properties are assigned to FEHM and the nodes of FEHM are centered within each element, except that nodes on 
the boundary elements are cut in half or into a fourth depending on if the element is an edge or corner node, respectively. The main restriction for using the Pollock method is that rectangular grids must be used. Given consistent interpolation functions for the numerical velocities, a streamline is computed uniquely. A streamline is obtained by expressing the velocities as the time derivatives of a particle position and solving the resulting differential equations (e.g. Shafer-Perini and Wilson, 1991). We assume that a velocity field obtained from our flow model has been discretized in a manner consistent with Figure 2-2.

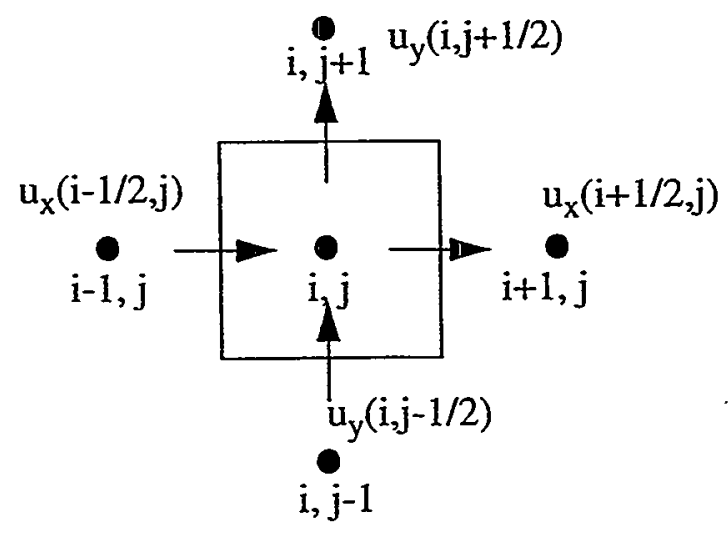

Figure 2-2. Schematic of the edge of cell velocities for a five-point block centered grid (SchaferPerini and Wilson, 1991).

The movement of a particle in each element is approximated by assuming that the velocity field within the box is time independent. Schafer-Perini and Wilson (1991) use a bilinear interpolator and show that it preserves mass and is accurate. For example, if we denote the $\mathrm{x}$ direction velocities on the two element faces normal to the $x$-direction ( $x$-direction faces) by $v_{x 1}$ and $v_{x 2}, v_{x}$ within the element is approximated by $v_{x}=a_{x} x+v_{x 1}$, where $a_{x}=\left(v_{x 2}-v_{x 1}\right) \Delta x$ and $\Delta x$ is distance between two $\mathrm{x}$-direction faces of the elements. The $\mathrm{x}$-coordinate is measured from the face with $x$-direction velocity $v_{x 1}$. Solving the differential equation $d x / d t=a_{x} x+v_{x 1}$ gives the distance of the particle as it moves within the element. The solution is (Pollock, 1988)

$$
x^{n+1}=\frac{\left[\left(a_{x} x^{n}+v_{x 1}\right) e^{a_{x} t}-v_{x 1}\right]}{a_{x}}
$$


where $x^{n+1}$ is the position within the cell after time $t$ (Figure 2-3). Analogous equations give $y^{n+1}$ and $\mathrm{z}^{\mathrm{n}+1}$. The method also provides the travel time as the particle moves through the domain. In

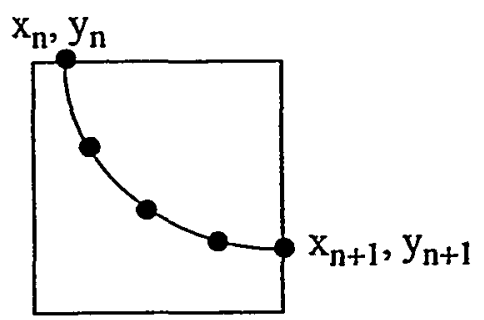

Figure 2-3. Solution of the Pollock method providing position within the cell after time t.

addition, we can also extract node based chemical properties such as mineralogy, reactive site concentration, and distribution coefficient along each streamline. These properties are necessary for reactive transport calculations.

For the steady state case, the Pollock (1988) method can easily be modified to perform a backward tracking of particles. Often, we are interested in a set of streamlines that arrive at a given location. For example, we may want to determine a set of streamlines that arrive at a sampling well. For this case, the sign of the velocities is simply reversed in the algorithm and the backward trajectory of each particle is calculated.

\subsubsection{Converting Streamlines into 1D Reactive Transport Simulations}

The Pollock (1988) method provides path lines and travel times along these path lines. The path lines then have to be converted into one-dimensional reactive transport simulations. In two dimensions, the process to convert streamlines into streamtubes is straightforward. The advantage of using streamtubes is that they carry a fixed volumetric flux (Batycky et al., 1996). For a twodimensional problem, a streamtube is simply the area between two streamlines. The total flux, $Q_{j}$, entering the streamtube can be determined from the flow model. Each streamtube can then be used to conduct a one-dimensional reactive transport simulation.

The methodology for converting streamlines into streamtubes is not nearly as straight: forward in three-dimensions. A rigorous local and globally mass conservative method to map 
fluxes along streamtubes requires maintaining the differential geometry of not just $2 \mathrm{D}$ crosssections but of 3D elements (pers. comm. Ginn 1998). Therefore it is advantageous to use streamlines instead of streamtubes since they are easily extended to 3D systems (Batycky et al., 1996). The disadvantage of using streamlines over streamtubes is that they do not carry an explicit volumetric flux. Therefore, we cannot simply calculate the appropriate flux entering each streamtube from the three-dimensional flow simulation as we do for the two-dimensional cases.

Thiele et al. (1995), Blunt et al. (1996), and Batycky et al. (1996) have developed a semiempirical approximation using the Pollock (1988) analytical method of calculating path lines to associate fluxes with particular streamlines. Their methods rely on decoupling the physics of the flow from the geologic heterogeneity. The influence of heterogeneity is captured with threedimensional streamlines, whereas the physics of the flow is captured in the appropriate 1D solution that is mapped along each streamline. Their methods have been applied to model oil recovery and have compared well to conventional finite difference codes and field measurements. Their methods are often more computationally efficient than conventional finite difference or finite element methods. The major advantage of the method is the ability to model sharp fronts with a fast and accurate technique. We apply an approach similar to Batycky et al. (1996) but utilize streamlines to model multicomponent reactive transport. A key question is to determine whether the method can work when nonlinear reactions are included. In addition, we want to simulate problems in which there are chemical heterogeneities. In chapter 4, we will compare the CDR and RST using a suite of two- and three-dimensional verification problems. Chapter 5 focuses on the effect of small scale heterogeneity on radionuclide transport through the Calico Hills unit which is one of the major barriers to radionuclide migration in the unsaturated zone. We utilize both the CDR model and the RST model for these calculations. In chapter 6 we will apply this approach to model the Unsaturated Zone Transport Test (UZTT) at Busted Butte.

A transport simulation is conducted for each streamline chosen from the flow model. Area and volume coefficients in the one-dimensional grid are adjusted so that the simulations will honor the travel times and distances along each streamline (Figure 2-4). In two dimensions, we compute 
the exact flowrate entering each streamtube from the flow solution. In three dimensions, we assign a flowrate to each streamline based on the fluxes at the inlet boundary of the flow solution. We choose the starting location of each streamline based on the problem of interest. Given the volumetric flow rate, $\mathrm{Q}_{\mathrm{j}}$, we can calculate the travel times, $\tau$, and $\mathrm{x}, \mathrm{y}$ and $\mathrm{z}$ positions along a streamline using the Pollock method; then the travel distance $\bar{x}$ along the streamline, flow-crosssectional area, $\mathrm{A}$, and pore volume, $\mathrm{V}$, for a given streamline using the following formulas:

$$
\begin{aligned}
& \bar{x}_{i+1, i}=\sqrt{\left(x_{i+1}-x_{i}\right)^{2}+\left(y_{i+1}-y_{i}\right)^{2}+\left(z_{i+1}-z_{i}\right)^{2}} \\
& \bar{v}_{i+1, i}=\frac{\bar{x}_{i+1}-\bar{x}_{i}}{\tau_{i+1}-\tau_{i}} \\
& A_{i+1, i}=\frac{Q_{j}}{\bar{v}_{i+1, i}} \\
& V_{\cdot i}=Q_{j}\left(\tau_{i+1}-\tau_{i}\right)
\end{aligned}
$$

where $\bar{v}=\frac{v}{\theta s}$ is the pore water velocity. Figure 2-4 describes how we use equations 2-33 to 2-36 to construct one-dimensional reactive transport simulations. In order to illustrate how the internodal area coefficients and node based volumes are used in each one-dimensional simulation, we write the discretized mass balance equation for grid block i (see Figure 2-4) for a chemical component:

$$
\frac{\partial}{\partial t}\left(c \rho_{l} V \theta \phi S_{l}\right)_{i}=\left[c \rho_{l} \bar{\nu} \phi s A-\rho_{l} \phi S_{l} D A \frac{\partial c}{\partial x}\right]_{i-1, \mathrm{i}}-\left[c \rho_{l} \bar{\nu} \phi S_{l} A-\rho_{l} \phi S_{l} D A \frac{\partial c}{\partial x}\right]_{i, \mathrm{i}+1}-(R V)_{i}(2-37)
$$

The grid spacing are adjusted along each segment of the one-dimensional simulation to honor the Courant $(u \Delta t / \Delta x<1)$ and grid Peclet $(u \Delta x / D<1)$ conditions. These "rules of thumb" were chosen since we are solving the reactive transport equations with an implicit scheme (Pinder and Gray, 1977). Segments of the streamline with faster velocities are assigned smaller grid spacings than slower moving portions of the streamline. Higher order schemes such as TVD would not require such stringent constraints; and codes such as MULTIFLO(Lichtner, 1998) or RT3D (Clement, 1997) could be substituted in place of FEHM to solve these one-dimensional reactive 
(a) Use Pollock method to generate streamlines in two- or three-dimensions

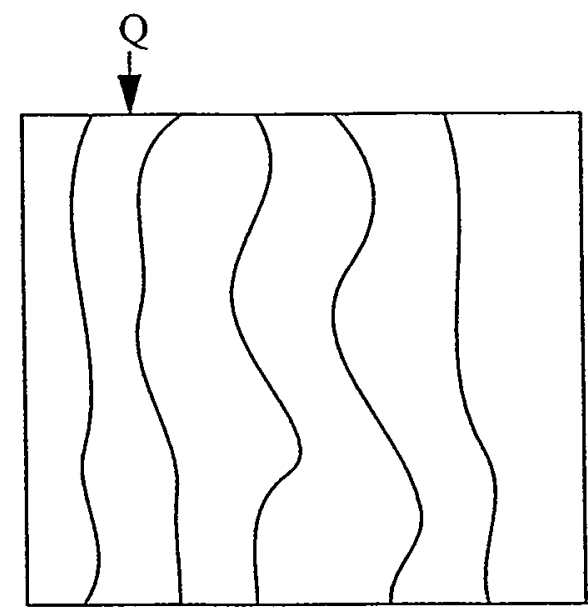

2D Streamlines/Streamtubes

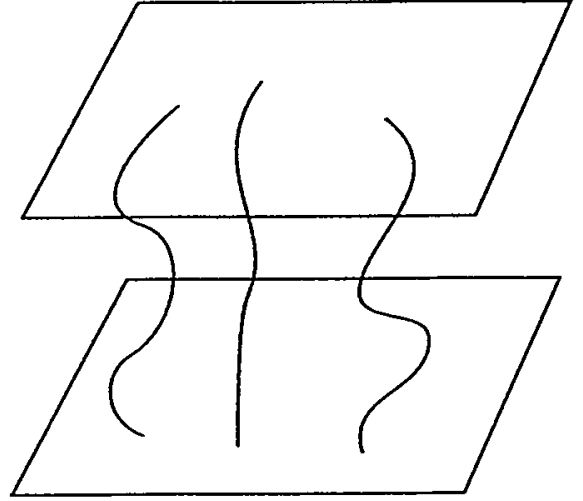

3D Streamlines

(b) From the Pollock(1988) method we extract travel time $(\tau)$, porosity $(\phi)$, saturation (s), and the relevant chemical properties (cp) at positions along the streamlines.

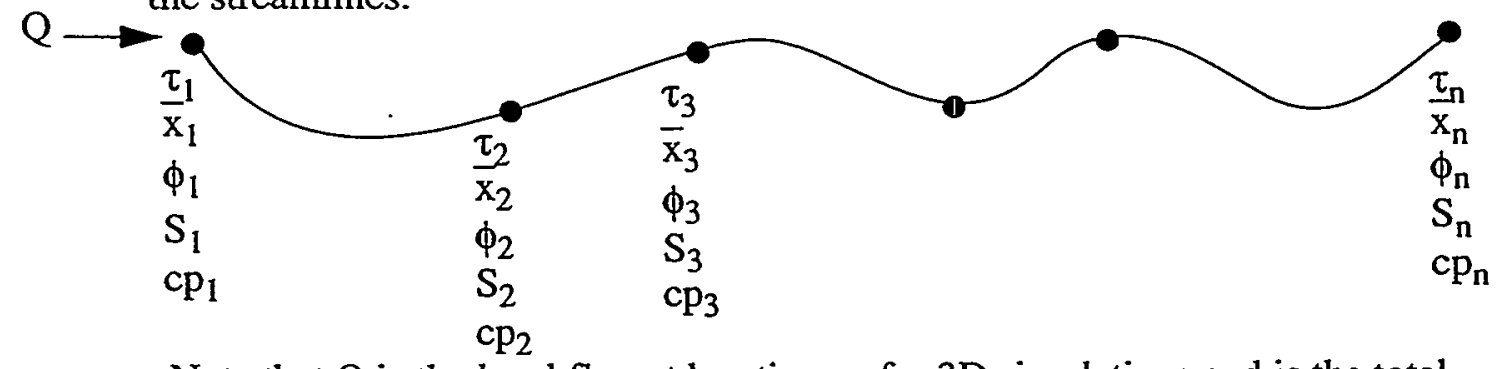

Note that $\mathrm{Q}$ is the local flux at location $\mathrm{x}_{1}$ for $3 \mathrm{D}$ simulations and is the total flux entering a streamtube for $2 \mathrm{D}$ simulations. Note that the streamtube of (a) is placed along its side.

(c) Using these properties we construct one-dimensional reactive transport simulations by adjusting the area and volume coefficients along with incorporating the appropriate

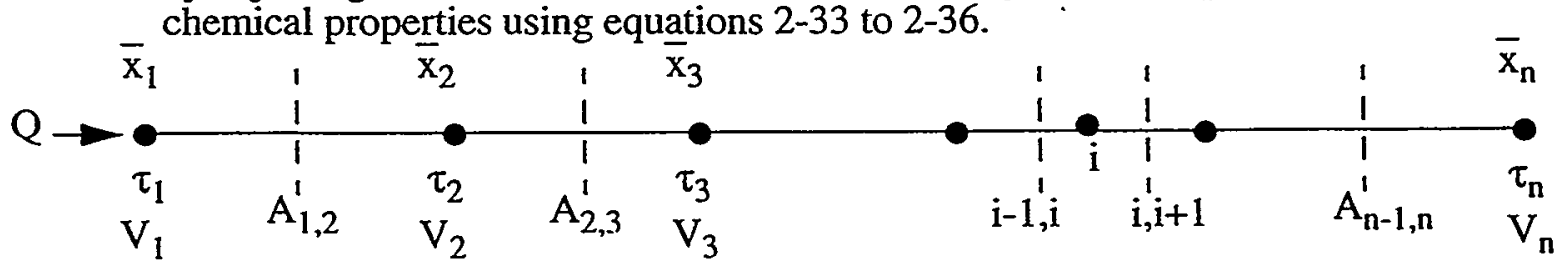

Figure 2-4. Schematic for constructing one-dimensional reactive transport simulations from the information obtained from the Pollock(1988) method. 
transport simulations. The concentration breakthrough at a given control plane can then be calculated using a flux weighted average of the concentration breakthrough curves from each of the streamtubes.

\subsubsection{Example of the RST Method for a Nonreactive Tracer}

The following example demonstrates the procedure of obtaining streamlines using the Pollock method and then converting these streamlines into one-dimensional transport simulations. A $10 \mathrm{~m} \times 10 \mathrm{~m} \times 10 \mathrm{~m}$ block was simulated with flow from the top of the block to the bottom. The flow is induced using constant pressure boundaries at the top and bottom of the model (see Figure 2-5). In the middle of the block, a $2 \mathrm{~m} \times 2 \mathrm{~m} \times 2 \mathrm{~m}$ low permeability region was inserted to create a bypass of flow around this region. A steady state flow field was calculated for this case using FEHM. Table 2-1 shows the parameters used for this simulation. We first compare the forward and

\begin{tabular}{|l|l|}
\hline \multicolumn{1}{|c|}{ Parameter } & \multicolumn{1}{c|}{ Value } \\
\hline \hline Pressure at top boundary & $1.0 \mathrm{MPa}$ \\
\hline Pressure at bottom boundary & $0.1 \mathrm{MPa}$ \\
\hline Low permeability region, $\mathrm{k}_{\text {low }}$ & $1 \times 10^{-19} \mathrm{~m}^{2}$ \\
\hline High permeability region, $\mathrm{k}_{\text {high }}$ & $1 \times 10^{-13} \mathrm{~m}^{2}$ \\
\hline Grid spacing & $1 \mathrm{~m}$ \\
\hline Time of pulse input at top boundary & 1 day \\
\hline Simulation time & 25 days \\
\hline longitudinal dispersivity & $0.1 \mathrm{~m}$ \\
\hline molecular diffusion coefficient & $1 \times 10^{-10} \mathrm{~m}^{2} / \mathrm{s}$ \\
\hline
\end{tabular}

Table 2-1. Parameter values for RST Example

reverse particle tracking methods to ensure that they provide identical results for this problem.

Figure 2-6 shows a three-dimensional picture of the 25 streamlines. As expected, the streamlines are perfectly symmetric around the $2 \mathrm{~m} \times 2 \mathrm{~m} \times 2 \mathrm{~m}$ low permeability block. In 


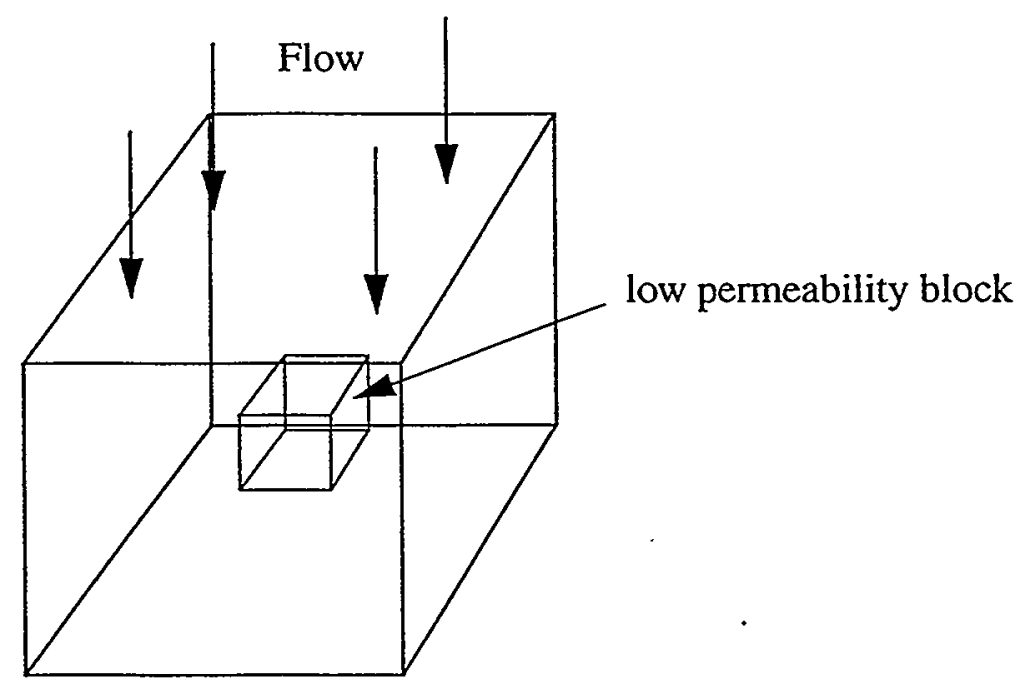

Figure 2-5. Three dimensional test problem.

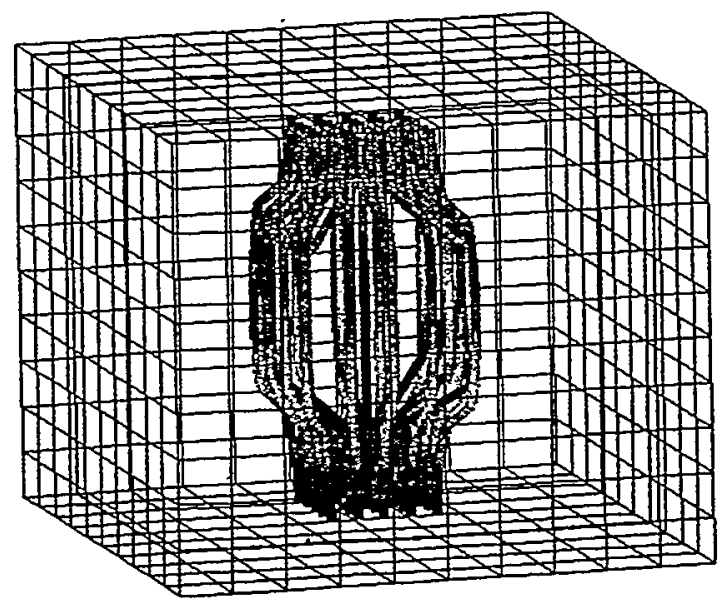

3D View

Figure 2-6. Plot of streamlines. Note that for this problem the 25 streamlines were released directly above the low permeability zone.

addition, the particle tracking algorithm produces identical results in both the backward and forward direction.

In this example problem, we run 25 one-dimensional simulations, each honoring travel times obtained from the 25 streamlines calculated by the Pollock(1988) method. In order to confirm that each one-dimensional simulation emulates a streamline with a given travel time, we 
compare the breakthrough curve at the exit of each streamline to an analytical solution for a fixed length pulse input given by Javendal et al. (1984):

$$
\begin{array}{ll}
\frac{C}{C_{0}}=F(x, t) & 0<t \leq t_{0} \\
\frac{C}{C_{0}}=F(x, t)-F\left(x, t-t_{0}\right) & t>t_{0} .
\end{array}
$$

where

$$
\begin{aligned}
& F(x, t)=\frac{1}{2} \operatorname{erfc}\left[\frac{x-\bar{v} t}{2(D t)^{1 / 2}}\right]+\left[\frac{\bar{\nu}^{2} t}{\pi D}\right]^{1 / 2} \cdot \exp \left[-\frac{(x-\bar{v} t)^{2}}{4 D t}\right] \\
& -\frac{1}{2}\left[1+\frac{\bar{v} x}{D}+\frac{u^{2} t}{D}\right] \exp \left[\frac{\bar{\nu} x}{D}\right] \operatorname{erfc}\left[\frac{x+\bar{v} t}{2(D t)^{1 / 2}}\right]
\end{aligned}
$$

where $t_{0}$ is the length of the pulse. Note that since we know the mean residence time through each streamline and the length of each streamline, we can calculate breakthrough curves using the analytical solution for each streamline to confirm that each one-dimensional numerical solution is correct. Specifically, we pick the pore water velocity, $\tilde{v}=$ length of streamline/ travel time. Figure 2-7 compares the breakthrough curves obtained from the analytical and numerical solutions. The analytical and numerical breakthrough curves plot directly on top of one another confirming that the numerical one-dimensional transport simulations are working properly. The RST curve is the flux weighted average of the 25 individual streamtube breakthrough curves. In this example, the 25 breakthrough curves can be broken down into three sets of travel times (see Figure 2-7). The first fifteen breakthrough curves represent the streamlines that completely bypass the low permeability unit resulting in the fastest travel times. The second set of breakthrough curves represents streamlines that partially intersect the low permeability unit and are therefore delayed. Finally, the last set of breakthrough curves are produced from streamlines that completely intersect the low permeability unit. The RST result is obtained by calculating a flux weighted average of the 25 individual breakthrough curves. This simple verification only confirms that each one-dimensional 


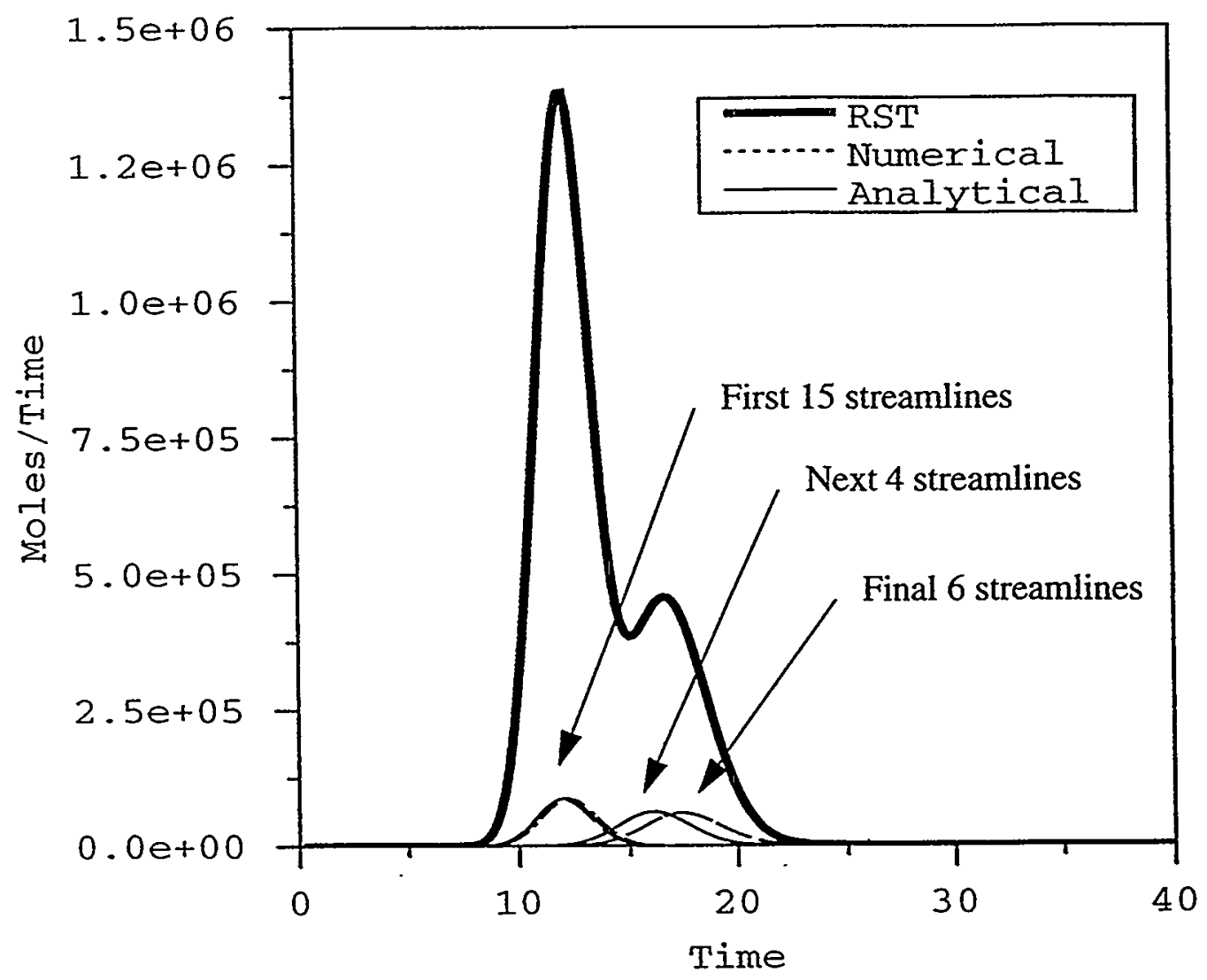

Figure 2-7. Comparison of one-dimensional transport simulations to analytical solutions.

simulation results in the correct breakthrough curve. In Chapter 4 , we present a set of verification problems that compares the RST and CDR methods for a variety of nonreactive and reactive transport problems. 


\section{VERIFICATION PROBLEMS FOR CDR}

\subsection{Summary}

The three verification problems in this chapter test the selective coupling technique and illustrate its power and flexibility for solving reactive transport problems. These examples are discussed in further detail in Robinson et al. (1999, in press). Example 1 is a hypothetical system in which six aqueous components participate in kinetic reactions. Example 1 illustrates the tradeoffs involved in selecting the level of coupling and the influence that problem size and the nature of the chemical system will have on the techniques. We chose a simple flow system and a fictitious chemical system to gain insight into the controlling factors of the coupling strategy because other complexities tend to obscure these factors in real applications. In Example 2, we present a reactive transport problem in which we model the coupled effects of a set of equilibrium speciation reactions and kinetic biodegradation and adsorption/desorption reactions. Therefore, this problem builds on the results of Example 1, testing the methods with a realistic reactive transport system that includes solute-rock interactions and a mixed kinetic-equilibrium formulation. Finally, Example 3 shows how selective coupling performs for a field application, namely the potential repository for high-level radioactive waste at Yucca Mountain, Nevada. The simulation utilizes an unstructured mesh with a large number of grid blocks and complex stratigraphy. This example illustrates the use of coupled normalization and selective coupling for significantly reducing the computational effort and memory requirements of the simulation. Thus, the lessons learned from Examples 1 and 2 provide the foundation for understanding the performance of the numerical techniques on a real field application. 


\subsection{Example 1:Kinetic Formulation for Reaction Among Aqueous Components}

In this first example we investigate the solution options for aqueous components coupled through kinetic reactions. Although many aqueous speciation reactions can be specified as equilibrium, there are exceptions, such as microbially facilitated redox reactions. Furthermore, in many applied reactive transport simulations, including those discussed in Examples 2 and 3 below, aqueous components undergo speciation reactions along with kinetic reactions such as competitive sorption. In these types of reactive systems, aqueous components often become tightly coupled through these kinetic reactions. Finally, even if the aqueous reactions are rapid, species with differing transport parameters such as dispersion or diffusion coefficients complicates the formulation of the system in terms of aqueous components and complexes (e.g. Lichtner, 1996). Alternatively, specifying aqueous components individually with different transport parameters is a way around this difficulty. Therefore, this test case was developed to demonstrate the method of selective coupling with a system in which all reactions are kinetic, even though for some reaction rates the problem could have been reformulated as an equilibrium reaction. The reaction system is a testbed for exploring the solution procedures that apply to more general reactive transport problems.

The chemical system chosen for study is the following system of reactive aqueous components:

$$
\begin{aligned}
& A+B \Leftrightarrow C \\
& C \Leftrightarrow D \\
& D+E \Leftrightarrow F
\end{aligned}
$$

with flow conditions, initial and boundary conditions illustrated in the schematic diagram of Figure 3-1. A one-dimensional, steady state flow system is used for this example, with initial concentrations of 0 (concentration units are arbitrary molar units) for all components except $E$, which initially is present everywhere in the column at concentration equal to 100 (0.1 for Cases $19-21)$. 


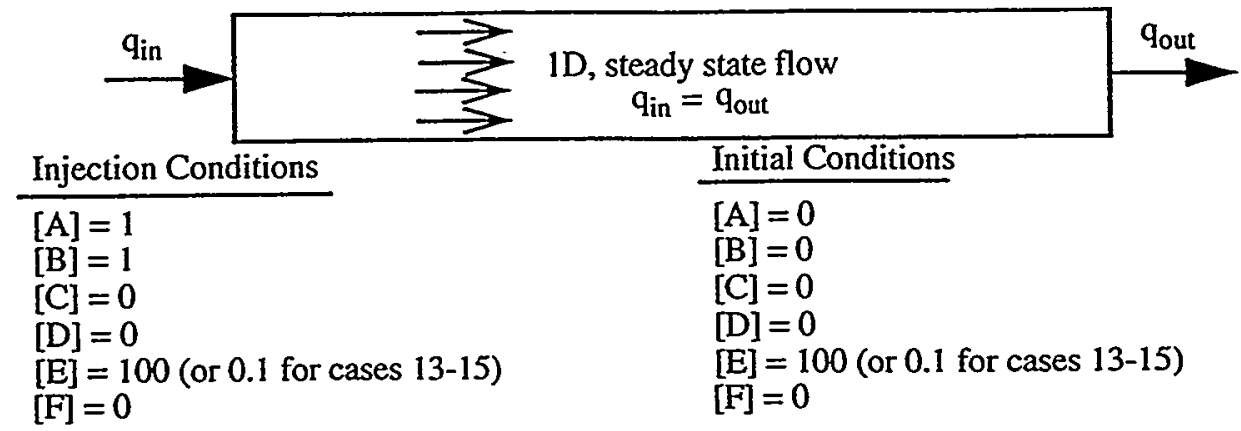

Figure 3-1. Schematic of the one-dimensional model domain used for Example 1.

Components $A$ and $B$ are injected at a concentration of 1 for all times, and component $E$ is injected at a concentration of 100 ( 0.1 for Cases 13-15). Rates of forward and reverse reactions are first order in all concentrations. For each reaction, the Damkohler number $D a=k_{r e v} \tau$, where $k_{r e v}$ is the reverse rate constant and $\tau$ is the travel time through the column, is the dimensionless group that controls the relative rate of reaction, whereby large $D a$ (of order 100 or more) implies nearequilibrium behavior, and $D a$ values of order 1 imply kinetically controlled reactions. In this exercise we vary $D a$ for the reactions to investigate the characteristics of the selective coupling method for obtaining convergent solutions with a minimum number of iterations.

In the first comparison (Cases 1-3), we assume $D a=1$ for all three reactions. The selective coupling method is then applied (groups of components solved simultaneously are denoted by enclosing them in parentheses below) with the following options for the transport iteration:

- Case 1: all six components solved individually (SIA-1), i.e. (A), (B), (C), (D), (E), and (F);

- Case 2: coupling of components as (A, B, C) followed by (D, E, F);

- Case 3: coupling of all six components (A, B, C, D, E, F).

For all cases the convergence criterion is the same, and the iterative technique at each time step must achieve overall convergence before proceeding to the next time step. We treat the 
convergence criterion as a parameter whose value is governed by the need for an accurate solution, and therefore we do not examine the impact of its value on the performance of the algorithms. The simulations are run with a maximum Courant number of 1 (except for the explicit examples that test the influence of time step, Cases 10-12 and 13-15) until the breakthrough of each component at the outlet has achieved a steady concentration. In Table 3-1, a summary of the numerical performance for this comparison shows that the computational work associated with a particular iteration increases as the degree of coupling increases, but that fewer iterations are required to obtain a converged solution. However, for the case of $D a=1$ for all three reactions, the uncoupled strategy yields somewhat better overall performance (lower total cpu time) than either coupling into 2 groups of 3 components or the fully coupled strategy. In fact, for this case with slow kinetics, operator splitting could be used with little mass balance error and should be even faster than the uncoupled strategy (van der Zee et al., 1989). The benefits of the selective coupling method are better illustrated in Cases 4-6, a comparison assuming $D a=100$ for the first and third reactions, and $D a=1$ for the second reaction. Convergence becomes problematic for the SIA-1 method, and the resulting number of iterations and the total cpu time increase dramatically. However, the nature of the reaction system makes the intermediate level of coupling sufficient for this problem. The rapid kinetics of Reaction 1 makes coupling of $A, B$, and $C$ necessary (the same is true for $D, E$, and $F$ due to Reaction 3), but the breaking into these two groups is possible because Reaction 2 imparts only a relatively weak coupling of $A, B$, and $C$ to $D, E$, and $F$. When $D a$ is increased to 1000 for the two reactions (Cases 7-9), the inadequacy of the uncoupled case becomes even more apparent.

To examine the influence of time step size on performance, Cases 10-12 and 13-15 were run with identical kinetic parameters as Cases 7-9, but with a maximum Courant number of 0.2 (Cases 10-12) or 5 (Cases 13-15). Cases 10-12 show that, as expected, lowering the time step makes the uncoupled case somewhat more competitive. However, the usefulness of coupling the aqueous components is best illustrated in Cases 13-15, in which convergence cannot even be 
obtained without coupling. Thus the coupling strategy allows larger time steps to be taken, whereas numerical stability becomes an issue without coupling.

\begin{tabular}{|c|c|c|c|c|c|}
\hline $\begin{array}{c}\text { Case } \\
\text { Number }\end{array}$ & Coupling & $\begin{array}{l}\text { Damkohler numbers for } \\
\text { the three reactions } \\
\left(D a_{1}, D a_{2}, D a_{3}\right)\end{array}$ & $\begin{array}{l}\text { Total \# of } \\
\text { Iterations }\end{array}$ & $\begin{array}{l}\text { cpu time per } \\
\text { Iteration (s) }\end{array}$ & $\begin{array}{l}\text { Total cpu } \\
\text { time (s) }\end{array}$ \\
\hline 1 & $(A)(B)(C)(D)(E)(F)$ & $(1,1,1)$ & 2915 & 0.073 & 212 \\
\hline $4: 2$ & $(A B C)(D E F)$ & $(1,1,1), \cdots$ & 2801 & 0.091 & 254 \\
\hline M3 & $(\mathrm{ABCD} \mathrm{CD})$, & $(1,1) \times 3)$ & $2206 \%$ & Wo14 & W \\
\hline 4 & $(A)(B)(C)(D)(E)(F)$ & $(100,1,100)$ & 6876 & 0.073 & 501 \\
\hline 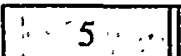 & $(\mathrm{ABC})(\mathrm{DEF})$ & $(100,1,100)$ & 2825 & 0.086 & 243 \\
\hline 66) & $(\mathrm{ABCD} \mathrm{BH})$ & $8(10031000)$ \% & $32681 \mathrm{~h}$ & 2014, & 36 \\
\hline 7 & $(A)(B)(C)(D)(E)(F)$ & $(1000,1,1000)$ & 80982 & 0.071 & 5752 \\
\hline 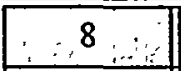 & $(A B C)(D E F)$ & $(1000,1,1000):$ & 2836 & 0.087 & 246 \\
\hline 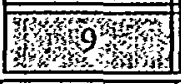 & 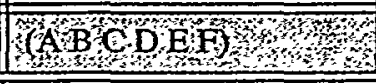 & $(1000,1,000), 10 \%$ & \$2 $2756 \%$ & 0,44 & 387 \\
\hline $10^{(1)}$ & $(A)(B)(C)(D)(E)(F)$ & $(1000,1,1000)$ & 36825 & 0.078 & 2868 \\
\hline$\because 11^{(1)} \cdots$ & $(\mathrm{A}: \mathrm{B}: \mathrm{C})(\mathrm{D} \cdot \mathrm{E} \cdot \mathrm{F})$ & $(1000,1,1000)$ & 10500 & 0.094 & 985 \\
\hline 6. & 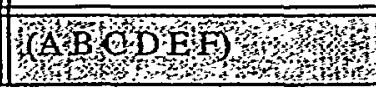 & 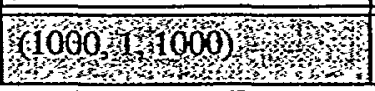 & W0488 & Wo 015 & W 242 \\
\hline $13^{(2)}$ & $(A)(B)(C)(D)(E)(F)$ & $(1000,1,1000)$ & $\begin{array}{c}\text { No } \\
\text { convergence }\end{array}$ & N/A & N/A \\
\hline $14^{(2)}$ & $(A B C)(D E F)$ & $(1000,1,1000)$ & $861^{\circ}$ & 0.093 & 80 \\
\hline 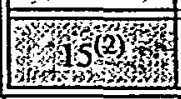 & M & $1000,1,1000)$, & 1584, & S 014 , & Wo4k \\
\hline 16 & $(A)(B)(C)(D)(E)(F)$ & $(1000,1000,1000)$ & 36792 & 0.073 & 2691 \\
\hline (n) & $(\mathrm{A} B \mathrm{~B})(\mathrm{DEF})$ & $(1000,1000,1000)$ & $\therefore 4064$ & 0.086 & $\because-350$ \\
\hline 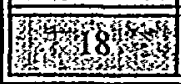 & 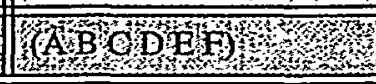 & $1000010001000)$ \% & W2313 & 100143 & 328 \\
\hline $19^{(3)}$ & $(A)(B)(C)(D)(E)(F)$ & $(1000,1000,1000)$ & 76863 & 0.073 & 5557 \\
\hline $20^{(3)}$ & $(\mathrm{A} B \mathrm{C})(\mathrm{D} E \mathrm{E})$ & $(1000,1000,1000)$ & 11058 & 0.083 & $\| 915$ \\
\hline 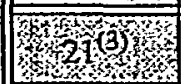 & $(\mathrm{AB} \mathrm{ODHATS})$ & $(1000,1000,1000), \mathrm{k}$ & $12766 \mathrm{6}$ & 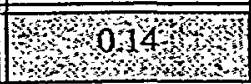 & 389 \\
\hline
\end{tabular}

Table 3-1. Computational efficiency of different levels of selective coupling: Example 1

(1) Same as Cases 7-9, except that the Courant number is 0.2.

(2) Same as Cases 7-9, except that the Courant number is 5 . 
In Cases $16-18, D a$ is increased to 1000 for Reaction 2 . At this point, the fully coupled strategy begins to slightly outperform the intermediate coupled strategy, as the reduction in the number of iterations required is enough to overcome the greater cpu time per iteration. Increasing the rate of Reaction 2 imparts a greater degree of coupling between the two sets of components, thereby causing the performance of the intermediate coupling strategy to begin to deteriorate.

The final comparison (Cases 19-21) illustrates that performance deteriorates markedly for even the intermediate degree of coupling when the injection and initial concentrations of $E$ are reduced from 100 to 0.1 . Although it is still possible to obtain a solution at the intermediate level of coupling, the number of iterations required and the total cpu time increase markedly for the uncoupled and intermediate coupling strategies. For concentration of $E$ of 100 , the forward reaction of Reaction 3 is essentially pseudo-first order in $D$. Reducing the concentration of $E$ to 0.1 changes the character of Reaction 3, making the concentrations of $A, B$ and $C$ much more closely linked to the concentrations of $D, E$ and $F$. These results illustrate that the proper strategy for selective coupling from the standpoint of computational efficiency depends on the nature of the chemical system, including the stoichiometry and kinetics of the reactions and the concentration values themselves.

For large-scale simulations, the memory required to obtain a solution, rather than the cpu time, may constrain the choice of solution strategy. For the selective coupling method, memory requirements can be severe for large simulations if many components need to be coupled to obtain a solution efficiently. We use this reaction system as an example to illustrate this point for a hypothetical three-dimensional, grid consisting of $N_{\text {cell }}$ cells. The Jacobian matrix requires a storage of $N_{d o f}^{2} N_{c e l l}$ for the $a$ submatrices in 2-25, and approximately $N_{d o f} N_{c o n} N_{c e l l}$ for the $t$ submatrices, or a total of $N_{d o f}\left(N_{d o f}+N_{c o n}\right) N_{c e l l}$, where $N_{c o n}$ is the average number of neighboring nodes connected to each node. In addition, incomplete factorization methods require the storage of a partial inversion of the Jacobian matrix in which, at a minimum, the $t$ submatrices 
become filled in, for a total storage of $N_{d o f}^{2}\left(N_{c o n}+1\right) N_{c e l l}$. The $N_{d o f}^{2}$ dependence of the storage places a premium on memory for simulations with a large number of grid points and high degrees of coupling. Table 3-2 shows the memory requirements for a specific example of a 100,000 node, hexahedral grid $\left(N_{\text {cell }}=100,000, N_{\text {con }}=6\right)$, for the various degrees of coupling studied in this reaction system. Furthermore, for more than one level of fill-in for the incomplete factorization, storage requirements are even more severe for the inversion matrix. Therefore, in large-scale simulations, memory requirements alone mandate that selective coupling be applied such that only the minimum degree of coupling needed for efficient convergence is used. For smaller problems, the decision of the degree of coupling hinges only on the cpu time issue, making the more robust full coupling of the solution an attractive alternative. The optimal use of computer resources is therefore dependent on the size of $N_{c e l l}$, and the nature of the chemical system, which in turn controls the efficiency of the numerical solution. Allowing the selection of partial levels of coupling allows the user to tailor the solution technique to the specific application..

\begin{tabular}{|l|l|l|l|}
\hline \multicolumn{1}{|c|}{ Coupling } & $\begin{array}{c}\text { Maximum } \\
N_{d o f}\end{array}$ & \multicolumn{1}{|c|}{$\begin{array}{c}\text { Size of Jacobian } \\
\text { (words) }\end{array}$} & \multicolumn{1}{|c|}{$\begin{array}{c}\text { Minimum size of partial } \\
\text { inversion of Jacobian (words) }\end{array}$} \\
\hline \hline (A) (B) (C) (D) (E) (F) & 1 & $7.0 \times 10^{5}$ & $7.0 \times 10^{5}$ \\
\hline (A B C) (D E F) & 3 & $2.7 \times 10^{6}$ & $6.3 \times 10^{6}$ \\
\hline (A B C D E F) & 6 & $7.2 \times 10^{6}$ & $2.5 \times 10^{7}$ \\
\hline
\end{tabular}

Table 3-2. Memory efficiency for a hypothetical 100,000 node simulation for different levels of selective coupling

\subsection{Example 2: CoNTA Transport Problem}

In the next example we consider a reactive transport problem from a natural system. Specifically, we model the transport of ${ }^{60} \mathrm{Co}$ in the presence of inorganic and organic chemical waste. This problem was proposed by Valocchi and Tebes-Stevens as a benchmark problem at the Workshop on Subsurface Reactive Transport Modeling held at Pacific Northwest National 
Laboratory (October, 1997). The reactions are described in more detail by Tebes-Stevens and Valocchi (1998). This reactive transport system has practical significance since soils and groundwater at DOE facilities have been contaminated by complex mixtures of radioactive, inorganic, and organic chemical wastes. In particular, Cobalt, as ${ }^{60} \mathrm{Co}$, is an radioactive contaminant that has been found migrating in the subsurface at several DOE facilities. The mobility of ${ }^{60} \mathrm{Co}$ has been greater than anticipated due to complexation with organic ligands such as EDTA and NTA. The objective of the analysis is to model the coupled effects of a set of equilibrium speciation reactions and kinetic biodegradation and adsorption/desorption reactions.

A one-dimensional column 10 meters in length was chosen for this simulation. The porosity, $\phi$, is 0.4 , the bulk rock density, $\rho_{b}$ is $1.5 \times 10^{3} \mathrm{~kg} / \mathrm{m}^{3}$, the pore water velocity is $1 \mathrm{~m} / \mathrm{hr}$, and the longitudinal dispersivity is $0.05 \mathrm{~m}$. Table 3-3 lists the components and concentrations of the background and injected fluid, while Table 3-4 shows the equilibrium speciation reactions.

The biodegradation of the complex HNTA ${ }^{2-}$ is represented by the following reaction:

$$
H N T A^{2-}+1.620 \mathrm{O}_{2}+1.272 \mathrm{H}_{2} \mathrm{O}+2.424 \mathrm{H}^{+} \rightarrow 0.576 \mathrm{C}_{5} \mathrm{H}_{7} \mathrm{O}_{2} \mathrm{~N}+3.120 \mathrm{H}_{2} \mathrm{CO}_{3}^{*}+0.424 \mathrm{NH}_{4}^{+} \text {. }
$$

The rate of substrate degradation $\left(\mathrm{R}_{\mathrm{HNTA}}\right)$ is modeled with multiplicative-Monod kinetics given by equation 2-12. The parameters used in equation $2-12$ are: $\mathrm{K}_{\mathrm{s}}=7.64 \times 10-7$ moles $/ \mathrm{L}, \mathrm{K}_{\mathrm{A}}=$ 6.25 $\times 10-6$ moles $/ \mathrm{L}$, and $\mathrm{q}_{\mathrm{m}}=1.407 \times 10-3$ moles/ NTA/g cells/hr (Tebes-Stevens and Valocchi, 


\begin{tabular}{|l|l|l|l|}
\hline \multicolumn{1}{|c|}{ Component } & \multicolumn{1}{|c|}{ Type } & \multicolumn{1}{c|}{ Pulse Concentration } & \multicolumn{1}{c|}{ Background Concentration } \\
\hline \hline $\mathrm{H}^{+}$ & aqueous & $\mathrm{pH}=6$ & $\mathrm{pH}=6$ \\
\hline $\mathrm{H}_{2} \mathrm{CO}_{3}$ & aqueous & $4.9 \times 10^{-7}$ moles $/ \mathrm{L}$ & $4.9 \times 10^{-7} \mathrm{moles} / \mathrm{L}$ \\
\hline $\mathrm{NH}_{4}^{+}$ & aqueous & 0.0 & 0.0 \\
\hline $\mathrm{O}_{2}$ & aqueous & $3.125 \times 10^{-5} \mathrm{moles} / \mathrm{L}$ & $3.125 \times 10^{-5} \mathrm{moles} / \mathrm{L}$ \\
\hline $\mathrm{NTA}^{3-}$ & aqueous & $5.23 \times 10^{-6}$ moles $/ \mathrm{L}$ & 0.0 \\
\hline $\mathrm{Co}^{2+}$ & aqueous & $5.23 \times 10^{-6}$ moles $/ \mathrm{L}$ & 0.0 \\
\hline Biomass & immobile & - & $1.36 \times 10^{-4} \mathrm{~g} / \mathrm{L}$ \\
\hline
\end{tabular}

Table 3-3. Components and concentrations of the background and injected fluid, CoNTA transport problem.

\begin{tabular}{|c|c|}
\hline Reaction & $\log$ Keq \\
\hline$H_{3} N T A \Leftrightarrow N T A^{3-}+3 H^{+}$ & 14.9 \\
\hline$H_{2} N T A^{-} \Leftrightarrow N T A^{3-}+2 H^{+}$ & 13.3 \\
\hline$H N T A^{2-} \Leftrightarrow N T A^{3-}+H^{+}$ & 10.3 \\
\hline$H_{2} O \Leftrightarrow O H^{-}+H^{+}$ & 14.0 \\
\hline$C o N T A^{-} \Leftrightarrow N T A^{3-}+\mathrm{Co}^{2+}$ & 11.7 \\
\hline $\operatorname{CoNTA}_{2}^{4-} \Leftrightarrow 2 N T A^{3-}+\mathrm{Co}^{2+}$ & 14.5 \\
\hline $\mathrm{CoOHNTA^{2- }} \Leftrightarrow \mathrm{NTA}^{3-}+\mathrm{Co}^{2+}+\mathrm{OH}^{-}$ & 0.5 \\
\hline $\mathrm{CoOH}^{+} \Leftrightarrow \mathrm{Co}^{2+}+\mathrm{OH}^{-}$ & -9.7 \\
\hline $\mathrm{Co}(\mathrm{OH})_{2} \Leftrightarrow \mathrm{Co}^{2+}+2 \mathrm{OH}^{-}$ & -22.9 \\
\hline $\mathrm{Co}(\mathrm{OH})_{3}^{-} \Leftrightarrow \mathrm{Co}^{2+}+2 \mathrm{OH}^{-}$ & -31.5 \\
\hline$N H_{3}+H^{+} \Leftrightarrow N H_{4}^{+}$ & -9.3 \\
\hline
\end{tabular}

Table 3-4. Equilibrium speciation reactions, CoNTA transport problem.

1998). The reaction rates for $\mathrm{O}_{2}, \mathrm{H}^{+}, \mathrm{H}_{2} \mathrm{CO}_{3}{ }^{*}$, and $\mathrm{NH}_{4}{ }^{+}$are proportional to the rate of substrate 
degradation. Using the stoichiometry of the above reaction, the appropriate rate expressions are:

$$
R_{O_{2}}=1.620 R_{H N T A^{2-}}, R_{H^{+}}=2.424 R_{H N T A^{2-}}, R_{H_{2} C O_{3}^{*}}=-3.120 R_{H N T A^{2 .}}, R_{N H_{4}^{+}}=1.620 R_{H N T A^{2 \cdot}}
$$

The net rate of microbial growth $\left(R_{\text {cells }}\right)$ is given by the synthesis rate (which is equal to the rate of degradation of the substrate multiplied by a yield coefficient) minus a first-order decay rate.

$$
R_{\text {cells }}=-Y R_{H N T A^{2}-}-b X_{m},
$$

where $Y$ is the yield coefficient and $b$ is the decay coefficient. The parameters $Y$ and $b$ were chosen to be $65.15 \mathrm{~g}$ cells/mole NTA and $0.00208 \mathrm{hr}^{-1}$, respectively.

The sorption reactions are represented by a linear kinetic model (Equation 2-10). In this problem, uncomplexed cobalt $\left(\mathrm{Co}^{2+}\right)$ and $\mathrm{CoNTA}^{-}$are retarded due to sorption. The distribution coefficients, $\mathrm{K}_{\mathrm{d}}$, for $\mathrm{Co}^{2+}$ and $\mathrm{CoNTA}^{-}$are assumed to be equal to $5.07 \times 10^{-3} \mathrm{~m}^{3} / \mathrm{g}$ and $5.33 \times 10^{-}$ ${ }^{4} \mathrm{~m}^{3} / \mathrm{g}$, respectively. Note that these distribution coefficients were selected to give retardation coefficients of 20 and 3 for $\mathrm{Co}^{2+}$ and $\mathrm{CoNTA}^{-}$, respectively. The $\mathrm{K}_{\mathrm{d}}$ values are chosen for demonstration of the numerical techniques, and do not necessarily represent realistic sorption behavior.

This test problem was broken up into two parts. In the first part of this problem, part A, the mass transfer coefficients for the sorption reactions were set to $1 \mathrm{hr}^{-1}$. In part $\mathrm{B}$, the mass transfer coefficient is increased to $1000 \mathrm{hr}^{-1}$. Setting the mass transfer coefficient to $1000 \mathrm{hr}^{-1}$ approximates equilibrium for the sorption reactions resulting in sharper concentration fronts. Figure 3-2 shows that the FEHM solution closely matches the solution presented by Valocchi and Tebes-Stevens (1998) for part A. Similar agreement is witnessed for part B.

As with the first example problem, the selective coupling method is then applied with the following options for the transport iteration to solve this problem:

- Case 1: all six aqueous components solved individually (SIA-1), i.e. $\left(\mathrm{H}^{+}\right),\left(\mathrm{H}_{2} \mathrm{CO}_{3}\right),\left(\mathrm{NH}_{4}{ }^{+}\right)$, 
Part (A): Solution with $1 \mathrm{mM}$ Buffer

Concentration profiles at end of column $(x=10 \mathrm{~m})$
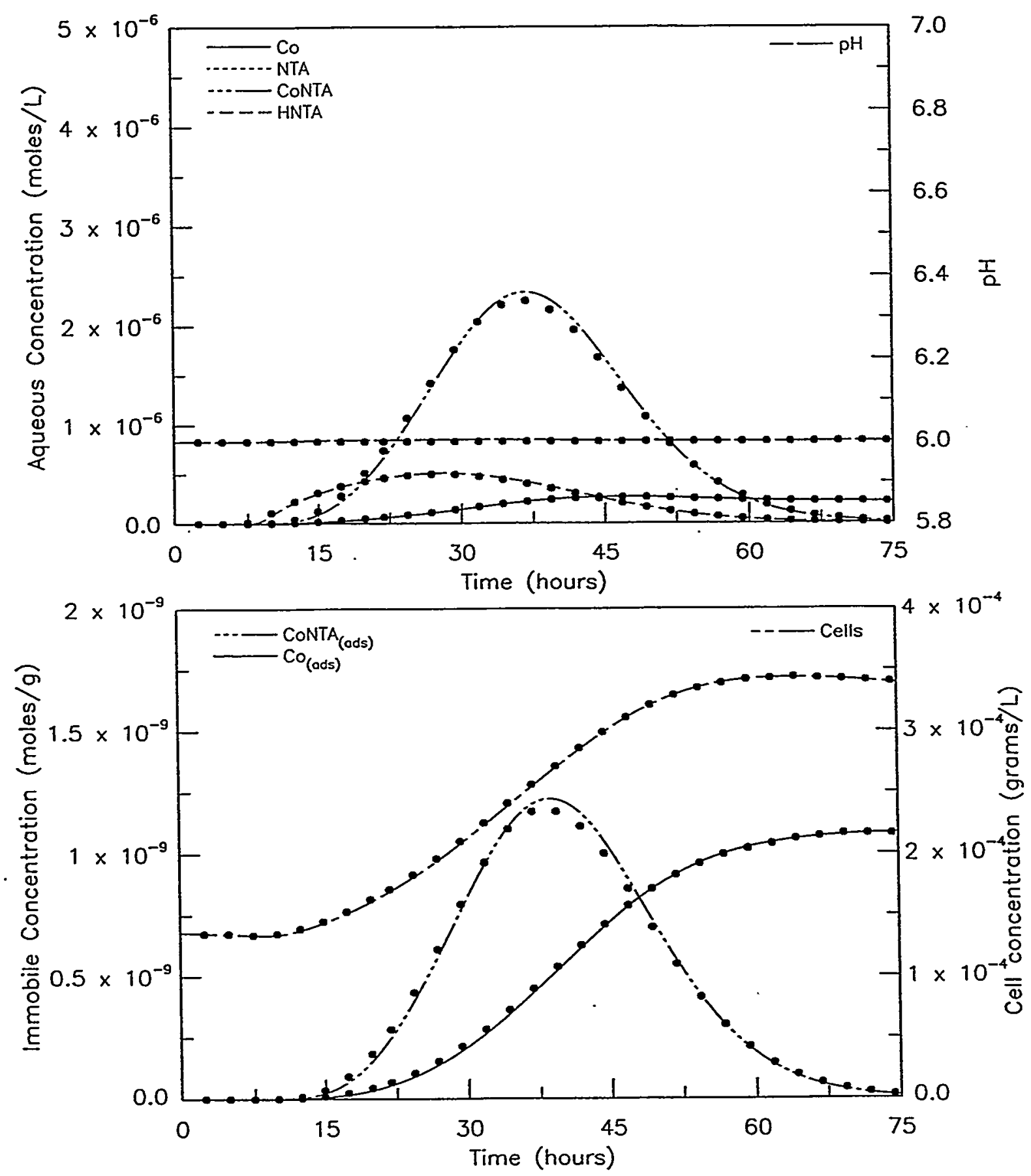

Figure 3-2. Comparison of FEHM (full circles) and Tebes-Stevens and Valocchi (1998) solution for part A. 
$\left(\mathrm{O}_{2}\right),\left(\mathrm{NTA}^{3-}\right)$, and $\left(\mathrm{Co}^{2+}\right)$. Biomass, CoNTA(ads) and $\mathrm{Co}(\mathrm{ads})$ solid components are solved individually;

- Case 2: coupling of aqueous components $\left(\mathrm{Co}^{2+}\right)$ and $\left(\mathrm{NTA}^{3-}\right)$ followed by $\left(\mathrm{H}^{+}\right),\left(\mathrm{H}_{2} \mathrm{CO}_{3}\right)$, $\left(\mathrm{NH}_{4}^{+}\right),\left(\mathrm{O}_{2}\right)$ which are solved individually. Biomass, CoNTA(ads) and $\mathrm{Co}$ (ads) solid components are solved individually;

- Case 3: coupling of all six aqueous components $\left(\mathrm{H}^{+}\right),\left(\mathrm{H}_{2} \mathrm{CO}_{3}\right),\left(\mathrm{NH}_{4}{ }^{+}\right),\left(\mathrm{O}_{2}\right),\left(\mathrm{NTA}^{3-}\right)$, and $\left(\mathrm{Co}^{2+}\right)$ (Global Implicit Method). Biomass, CoNTA(ads) and $\mathrm{Co}(\mathrm{ads})$ solid components are solved individually.

Recall, that the reaction rates for cobalt and NTA are coupled to one another due to the competitive sorption reactions, whereas, the other aqueous components are not strongly coupled to one another due to kinetic reactions. Thus, we chose to couple only cobalt and NTA in Case 2. Table compares the three cases..

\begin{tabular}{|l|l|l|l|l|l|l|}
\hline $\begin{array}{c}\text { Problem } \\
\text { Set }\end{array}$ & \multicolumn{2}{|c|}{$\begin{array}{c}\text { CASE 1 } \\
\text { SIA-1 }\end{array}$} & \multicolumn{2}{c|}{$\begin{array}{c}\text { CASE 2 } \\
\text { Selective Coupling: Co } \\
\text { and NTA }\end{array}$} & \multicolumn{2}{c|}{$\begin{array}{c}\text { CASE 3 } \\
\text { Global Implicit Method }\end{array}$} \\
\hline & $\begin{array}{c}\text { avg no. of } \\
\text { iterations per } \\
\text { time step }\end{array}$ & $\begin{array}{c}\text { total CPU } \\
\text { time (s) }\end{array}$ & $\begin{array}{c}\text { avg no. of } \\
\text { iterations per } \\
\text { time step }\end{array}$ & $\begin{array}{c}\text { total CPU } \\
\text { time (s) }\end{array}$ & $\begin{array}{c}\text { avg no. of } \\
\text { iterations per } \\
\text { time step }\end{array}$ & $\begin{array}{c}\text { total CPU } \\
\text { time (s) }\end{array}$ \\
\hline \hline Part A & 3.0 & 339 & 2.8 & 320 & 2.8 & 436 \\
\hline Part B & 7.5 & 888 & 3.5 & 417 & 3.5 & 525 \\
\hline
\end{tabular}

Table 3-5. Computational efficiency, CoNTA transport problem.

For part (A), Case 1 and Case 2 are comparable in total CPU time, whereas Case 3 takes longer to run. In part (A), the $\partial R_{i} / \partial C_{j}$ are small due to the slow sorption kinetic parameters. Therefore, Case 1 performs as well as Case 2. Since SIA-1 (Case 1) and selective coupling of cobalt and NTA (Case 2) solve smaller equation sets than the global implicit method (Case 3), these methods are more efficient for part A. For this problem, SIA-1 method solves six $N_{c} \times N_{c}$ 
matrices. Selective coupling solves one $2 N_{c} \times 2 N_{c}$ matrix (for cobalt and NTA), and four $N_{c} \mathrm{x}$ $N_{c}$ matrices for the remaining aqueous components. The global implicit method solves one $6 N_{c}$ $\mathrm{x} 6 N_{c}$ matrix solving for all the aqueous components simultaneously. All three methods solve for the solids individually. In part B, the coupling between cobalt and NTA is more important due to the faster kinetic rates. SIA-1 requires, on average, 7.5 iterations per time step, whereas, selective coupling and the global implicit methods require only 3.5 iterations per time step. Although the time for each iteration is still faster for SIA-1 than for the other two methods, more iterations per time step are required for convergence for SIA-1 resulting in larger run times. The SIA-1 method requires a large number of iterations per time step to converge since the cross derivative terms $\left(\partial R_{i} / \partial C_{j}\right)$ neglected by SIA-1 are now significant in part (B). Selective coupling outperforms the global implicit method since it includes the necessary cross derivative terms, but still neglects most of the other insignificant cross derivative terms computed by the global implicit solution.

\subsection{Example 3: ${ }^{237} \mathrm{~Np}$ Reactive Transport at Yucca Mountain}

In this example, we simulate the unsaturated zone transport of ${ }^{237} \mathrm{~Np}$ from the potential high-level waste repository at Yucca Mountain. For a detailed description of the hydrologic and geochemical processes affecting the migration of ${ }^{237} \mathrm{~Np}$, see Viswanathan et al.(1998). This problem demonstrates the selective coupling method for a complex, unstructured grid and a reactive transport system with speciation and competitive ion exchange. By choosing a model with a relatively large and complex grid, and mixed equilibrium-kinetic chemical formulation, we examine the numerical performance on a system for which the model was developed, namely large field applications. The ion exchange reactions require that solid components be coupled with aqueous components. Therefore, this problem also demonstrates the efficiency of coupled normalization. For the purposes of the current study we will briefly summarize the problem and then demonstrate the performance of the method. 
The domain selected for the transport calculations is a two-dimensional, East-West cross section through Yucca Mountain at the location of the potential repository. Figure 3-3a shows the
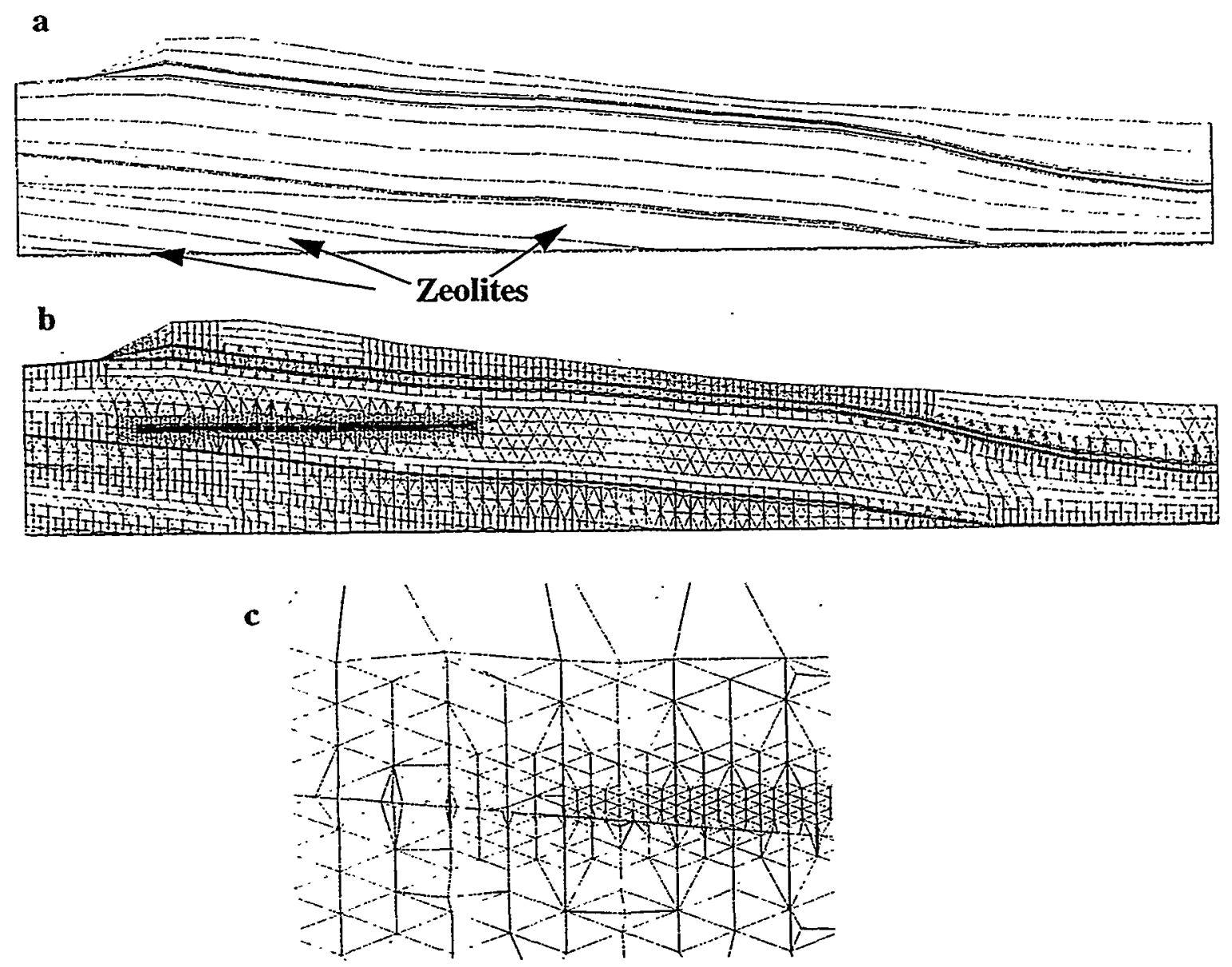

Figure 3-3. Stratigraphy and numerical grid for the Neptunium reactive transport problem (Example 3). a) dipping stratigraphy, including the location of zeolitic horizons; b) numerical grid of full model domain; c) close-up of grid at the edge of the potential repository.

East-dipping stratigraphy at this cross section, including the zeolitic horizons important to the transport of neptunium. The finite element grid for the entire cross section is shown in Figure 3-3b and $c$. The unstructured grid captures the complex stratigraphy at the site scale, while also allowing transport near the potential repository to be captured at a grid spacing of about $3 \mathrm{~m}$ resulting in a mesh with 7070 spatial nodes. For details on the hydrologic models, parameters, and infiltration fluxes used to investigate ${ }^{237} \mathrm{~Np}$ transport, see Viswanathan et al.(1998). In the present study, the 
nonisothermal effects examined in that paper are ignored in favor of an isothermal model, so that the chemical transport processes and numerical schemes can be examined more directly.

The geochemical processes that strongly affect ${ }^{237} \mathrm{~Np}$ migration include: solubility-limited release of ${ }^{237} \mathrm{~Np}$ from the repository, aqueous speciation of neptunium into non-sorbing carbonate/ hydroxy complexes and the sorbing $\mathrm{N}_{p} \mathrm{O}_{2}^{+}$cation, sorption of ${ }^{237} \mathrm{~Np}$ onto the zeolitic tuffs via an ion exchange mechanism, and radioactive decay. Solubilities for groundwater representative of Yucca Mountain were obtained from Efurd et al.(1996). We model the precipitation-dissolution of neptunium at the repository using equation 2-16, with the kinetic parameter value chosen to ensure that the reaction proceeds to equilibrium. The aqueous speciation reactions for Neptunium are given in Table 3-6.

The three total aqueous components chosen to model these reactions are $\mathrm{Np}_{2}^{+}, \mathrm{HCO}_{3}^{-}$, and $\mathrm{H}^{+}$. To simulate the sorption of $\mathrm{NpO}_{2}^{+}$cation, we couple the speciation results presented in Table with an ion exchange model for $\mathrm{Np}_{2} \mathrm{O}_{2}^{+}$. The following set of ion exchange reactions are assumed in the present study:

$$
\begin{array}{ll}
\mathrm{NpO}_{2}^{+}+t A l-N a^{+} \Leftrightarrow t A l-N p \mathrm{O}_{2}^{+}+N \dot{a}^{+} & \log _{10} \mathrm{~K}=-1.58 \\
\mathrm{Ca}^{2+}+2 t A l-N a^{+} \Leftrightarrow(2 t A l)-\mathrm{Ca}^{2+}+2 N a^{+} & \log _{10} \mathrm{~K}=1.5
\end{array}
$$

where $t A l$ represents a tetrahedral aluminum sorption site (Viswanathan et al., 1998). We model these reactions with kinetic rate laws. The main chemical influences on the extent of sorption onto the zeolitic tuffs according to this model are $\mathrm{pH}$ (controlled by the speciation reactions) and the concentrations of the competing cations in the sorption reactions, $\mathrm{Na}^{+}$and $\mathrm{Ca}^{2+}$. This problem consists of five total aqueous components: $\mathrm{Np}_{2}^{+}, \mathrm{HCO}_{3}^{-}, \mathrm{H}^{+}, \mathrm{Na}^{+}$, and $\mathrm{Ca}^{2+}$ and four immobile com- 
ponents: $\mathrm{Np}(\mathrm{s}), t \mathrm{tAl}-\mathrm{Np} \mathrm{O}_{2}^{+},(2 t \mathrm{Al})-\mathrm{Ca}^{2+}, t \mathrm{Al}-\mathrm{Na}^{+}$.

\begin{tabular}{|c|c|}
\hline Reaction & $\log \mathrm{Keq}$ \\
\hline $\mathrm{H}_{2} \mathrm{CO}_{3}(\mathrm{aq}) \Leftrightarrow \mathrm{HCO}_{3}^{-}+\mathrm{H}^{+}$ & -6.34 \\
\hline $\mathrm{HCO}_{3}^{-} \Leftrightarrow \mathrm{CO}_{3}^{2-}+\mathrm{H}^{+}$ & 10.33 \\
\hline$H_{2} \mathrm{O} \Leftrightarrow O H^{-}+H^{+}$ & -14.17 \\
\hline $\mathrm{NpO}_{2}^{+}+\mathrm{H}_{2} \mathrm{O} \Leftrightarrow \mathrm{NpO}_{2}(\mathrm{OH})^{0}(\mathrm{aq})+\mathrm{H}^{+}$ & 8.9 \\
\hline $\mathrm{NpO}_{2}^{+}+2 \mathrm{H}_{2} \mathrm{O} \Leftrightarrow \mathrm{NpO}_{2}(\mathrm{OH})_{2}^{-}+2 \mathrm{H}^{+}$ & 20.2 \\
\hline $\mathrm{NpO}_{2}^{+}+\mathrm{HCO}_{3}^{-} \Leftrightarrow \mathrm{NpO}_{2}\left(\mathrm{CO}_{3}\right)^{-}+\mathrm{H}^{+}$ & 5.73 \\
\hline $\mathrm{NpO}_{2}^{+}+2\left(\mathrm{HCO}_{3}^{-}\right) \Leftrightarrow \mathrm{NpO}_{2}\left(\mathrm{CO}_{3}\right)_{2}^{3-}+2 \mathrm{H}^{+}$ & 13.66 \\
\hline $\mathrm{NpO}_{2}^{+}+3\left(\mathrm{HCO}_{3}^{-}\right) \Leftrightarrow \mathrm{NpO}_{2}\left(\mathrm{CO}_{3}\right)_{3}^{5-}+3 \mathrm{H}^{+}$ & 22.49 \\
\hline
\end{tabular}

Table 3-6. Equilibrium speciation reactions, Neptunium transport problem.

Figure 3-4 shows the mass flux breakthrough at the water table of Neptunium for a solution of $\mathrm{pH}=8,\left[\mathrm{Na}^{+}\right]=5.43 \times 10^{-3} \mathrm{M}$, and $\left[\mathrm{Ca}^{2+}\right]=2.91 \times 10^{-4} \mathrm{M}$ (curve a). For reference, the breakthrough of a solute with the same release concentration but no sorption on the zeolites is also shown (curve d). Retardation in the zeolitic tuffs is an important process for predicting the migration of Neptunium through the unsaturated zone. With regard to the solution technique, this example illustrates the use of the coupled normalization step for properly linking the aqueous and immobile species in the numerical solution. To demonstrate this, we performed a series of simulations with different coupling strategies, examining the relative performance for two different reaction rates: 


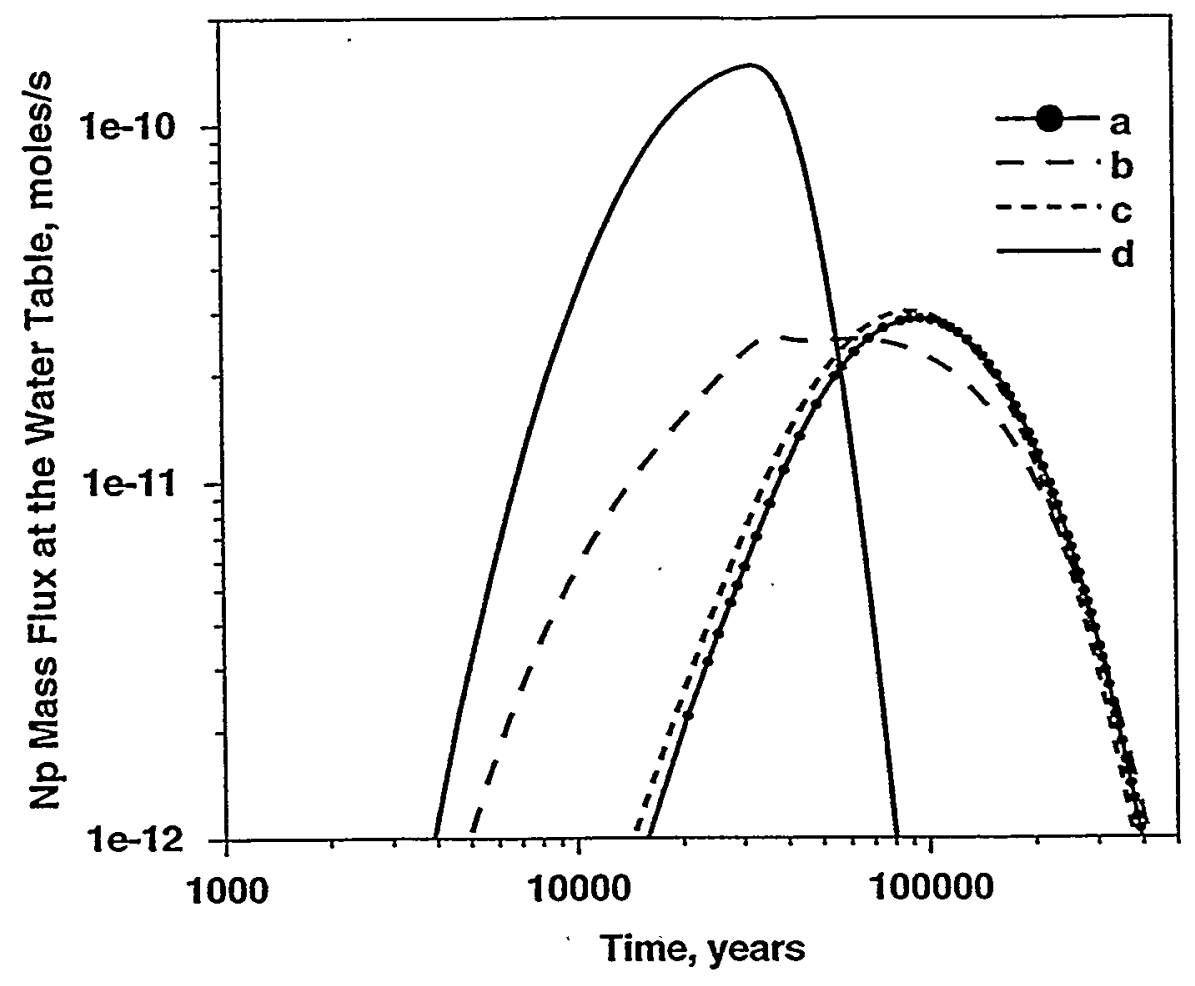

Figure 3-4. Neptunium breakthrough mass flux at the water table. Curve a) equilibrium sorption reactions; b) slow reaction kinetics for sorption reactions (Case 1); c) rapid reaction kinetics for sorption reactions (Case 2); d) nonreactive tracer with the same repository release rate as a-c.

- Case 1a: The components $\mathrm{HCO}_{3}^{-}, \mathrm{Np}(\mathrm{s})$ and $\mathrm{H}^{+}$were solved for individually. The components $\mathrm{N}_{p} \mathrm{O}_{2}^{+}, \mathrm{Ca} 2+, \mathrm{Na}+, t \mathrm{tAl}-\mathrm{Np} \mathrm{O}_{2}^{+},(2 t \mathrm{Al})-\mathrm{Ca}^{2+}$, and $t \mathrm{Al}-\mathrm{Na}^{+}$were run with selective coupling and coupled normalization.

- Case 1b: Same as Case 1a, but without coupled normalization.

- Case 1c: All components solved individually (SIA-1), without coupled normalization.

- Cases 2a-2c: These runs employ identical solution strategies as Cases 1a-1c, but the rate constants are an order of magnitude larger than those of Case 1.

The ion-exchange reactions in this example approach equilibrium behavior for Case 2 (Curve $c$ in Figure 3-4), but show significant deviations from equilibrium behavior for the slower 
kinetics of Case 1 (Curve b). Table 3-7 shows that the numerical performance of the coupled normalization and selective coupling method (Case 1a) was superior to either the selective coupling method without coupled normalization (Case 1b) or the SIA-1 method (Case 1c). In addition, if the kinetics are faster (Case 2), the performance degrades further for the cases without coupled normalization. Although it is difficult to compute a global value of $\mathrm{Da}$ for a complex system with varying velocities, the comparison between Cases 1 and 2 can be viewed in terms of the closeness to equilibrium reaction behavior. The more rapid the kinetics, the greater is the need to employ selective coupling and, in this example, especially coupled normalization. The $b$ and $c$ cases only become competitive with the preferred coupling strategy (a) when the kinetics are slow enough to result in significant deviations from equilibrium behavior. In Case 2, the b and c strategies can be made to produce results that are not significantly different than the coupled normalization case, but at great computational expense. Therefore, as in the previous examples, the numerical techniques developed in the present study are needed for the efficient solution of systems of chemical species coupled through kinetic reactions, especially for cases in which the reaction rates are rapid.

\begin{tabular}{|c|c|c|c|c|}
\hline Case & Description & $\begin{array}{c}\text { Total \# of } \\
\text { Time Steps }\end{array}$ & $\begin{array}{c}\text { Total \# of } \\
\text { Iterations }\end{array}$ & $\begin{array}{c}\text { Total cpu } \\
\text { time (hr) }\end{array}$ \\
\hline \hline la & $\begin{array}{c}\text { Selective coupling and Coupled Normalization, } \\
\text { slow kinetics }\end{array}$ & 125 & 385 & 1.01 \\
\hline \hline Ib & Selective coupling only, slow kinetics & 486 & 2192 & 2.8 \\
\hline \hline Ic & SIA-1, slow kinetics & 515 & 2433 & 2.39 \\
\hline $2 \mathrm{a}$ & $\begin{array}{c}\text { Selective coupling and Coupled Normalization, } \\
\text { rapid kinetics }\end{array}$ & 125 & 386 & 1.06 \\
\hline \hline $2 \mathrm{~b}$ & Selective coupling only, rapid kinetics & 5379 & 20103 & 25 \\
\hline \hline $2 \mathrm{c}$ & SLA-1, rapid kinetics & 6463 & 24005 & 23.3 \\
\hline
\end{tabular}

Table 3-7. Computational efficiency, Neptunium transport problem. 


\section{VERIFICATION PROBLEMS FOR RST}

\subsection{Summary}

In this chapter two- and three-dimensional verification problems are constructed to compare the reactive streamtube (RST) and the conventional convection-dispersion-reaction (CDR) methods. We first compare the methods for two- and three-dimensional nonreactive transport problems for several simple flow scenarios: flow through two layers with different permeabilities, a simple flow bypass problem, and flow through a random permeability field. We show that RST is often an accurate method for obtaining the solute flux at a given control plane. However there are exceptions in which transverse dispersion is one of the dominant processes in the system and cannot be neglected causing RST to fail. For the next set of test problems, we include a spatially variable linear retardation factor to simulate the sorption of a contaminant. Specifically, each streamline will encounter a different amount of retardation since the distribution . coefficient, $\mathrm{K}_{\mathrm{d}}$, varies along each streamline and also among streamlines. Finally, we test RST for a multicomponent reactive transport problem. For the reactive transport simulations, the selective coupling algorithm developed in Chapter 2 is used in the both the CDR and RST methods. We simulate the transport of the radionuclide ${ }^{237} \mathrm{~Np}$ through a random field of permeability. Aqueous ${ }^{237} \mathrm{~Np}$ forms numerous aqueous complexes some of which sorb to the zeolitic tuffs that are present in the Calico Hills. The effect of zeolitization on ${ }^{237} \mathrm{~Np}$ migration is another process that we will investigate. Highly zeolitized areas will retard ${ }^{237} \mathrm{~Np}$ but these regions are also less permeable than the nonzeolitized regions possibly allowing some of these areas to be bypassed. The full suite of test problems serves to demonstrate the accuracy, computational efficiency and limitations of the RST method. 


\subsection{D Nonreactive Transport}

The first three verification problems compare the CDR, PTRK, and RST methods for a nonreactive tracer. In all three examples, the problem domain is a $10 \mathrm{~m}$ by $10 \mathrm{~m}$ block. The top and bottom boundaries are constant pressure boundaries, whereas the right hand side and left hand side of the domain are no flow boundaries. The top and bottom boundaries were both set at $0.1 \mathrm{MPa}$. Therefore, the flow in these problems is gravity driven. A steady state flow field is calculated through the fully saturated block before the tracer input is started. The porosity, $\phi$, and the bulk rock density, $\rho_{b}$ were set equal 0.27 and $2500 \mathrm{~kg} / \mathrm{m}^{3}$, respectively, for all the simulations in this chapter. The streamlines were chosen such that each streamtube contained approximately the same water flux. These fluxes were obtained from the two-dimensional flow model as described in Chapter 2. Simulations that did not utilize streamlines with approximately equal flux gave poor results. In section 4.2 .3 we provide an example of how the number of streamtubes affects the flux averaged breakthrough curve provided by the RST method. Note that the number of streamlines necessary to accurately represent the flow field is strongly dependent on the heterogeneity of the problem domain.

\subsubsection{D Vertical Layer Problem}

In this example, the block is divided up into a low permeability zone and a high permeability zone (Figure 4-1). Nonreactive tracer is injected along the top of the model for 1000 days. Table 4-1 shows the parameters used in this simulation. The flux averaged concentration along the bottom boundary is compared for the CDR and RST method. Figure 4-2 shows that the CDR, PTRK and RST produce very similar results for this problem indicating that transverse dispersion is not an important process for these parameter values. The first peak represents the flux that travels through the high permeability region. The majority of the tracer flux flows through this region. The second 


\begin{tabular}{|l|l|}
\hline \multicolumn{1}{|c|}{ Parameter } & \multicolumn{1}{c|}{ Value } \\
\hline \hline Low permeability region, $\mathrm{k}_{\text {low }}$ & $3 \times 10^{-13} \mathrm{~m}^{2}$ \\
\hline High permeability region, $\mathrm{k}_{\text {high }}$ & $1 \times 10^{-12} \mathrm{~m}^{2}$ \\
\hline Velocity in low permeability region & $3.4 \times 10^{-4} \mathrm{~m} / \mathrm{s}$ \\
\hline Velocity in high permeability & $1.1 \times 10^{-3} \mathrm{~m} / \mathrm{s}$ \\
\hline Time of pulse input at top boundary & 1 day \\
\hline Simulation time & 15 days \\
\hline longitudinal dispersivity (for $\mathrm{RST}, \mathrm{CDR})$ & $0.1 \mathrm{~m}$ \\
\hline transverse dispersivity (CDR) & $0.01 \mathrm{~m}$ \\
\hline molecular diffusion coefficient & $1 \times 10^{-10} \mathrm{~m}^{2} / \mathrm{s}$ \\
\hline
\end{tabular}

Table 4-1. Parameter values for 2D vertical layer problem

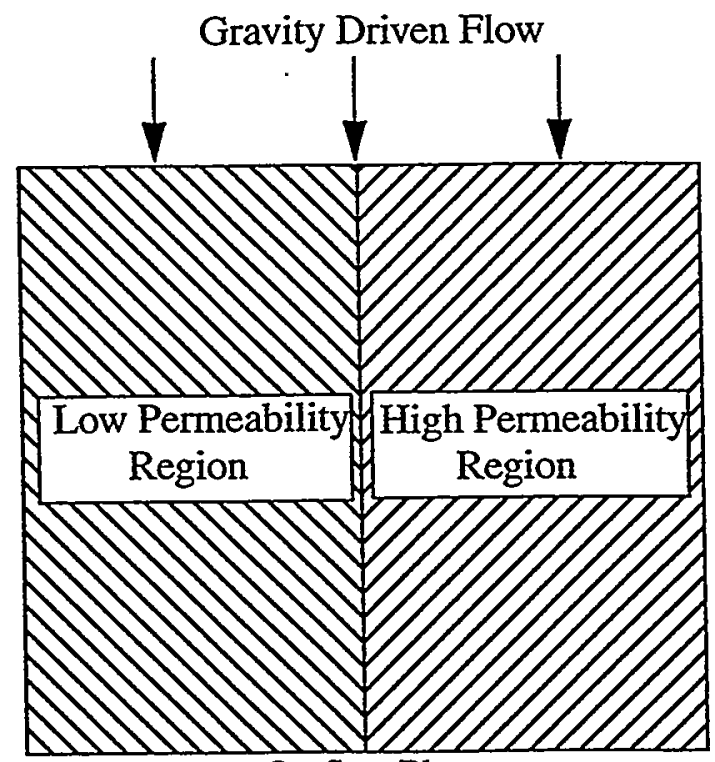

Outflow Plane

Figure 4-1. Two-dimensional flow with vertical layers.

peak is produced from tracer that travels through the low permeability region. Table 4-2 compares the CPU times among the three methods. Note that all computations were performed on a Sun Sparc 2 Workstation and that the timings were done using a SUN timing utility. As mentioned in Chapter 2, with the RST method, the grid spacing is adjusted to honor the Courant $(u \Delta t / \Delta x<1)$ and 
grid Peclet $(u \Delta x / D<1)$ conditions. With the CDR method we also attempt to honor these conditions. For the two- and three-dimensional CDR simulations, a grid refinement approach was used to confirm that the time step and grid spacing were sufficient to prevent significant numerical dispersion while not being too stringent so as to give inefficient CPU times. For this example, a maximum CDR time step of 0.05 days and a grid spacing of $0.1 \mathrm{~m}$ (in the $\mathrm{x}$ and $\mathrm{y}$ directions) were used. RST is more efficient than the CDR for this case. For this special case, only 2 streamtubes, one for each layer, are enough to characterize the flow field. The efficiency of PTRK is not relevant to our study since the method cannot model multicomponent reactive transport. Note that the initial distribution of particles at the inlet in the PTRK method are distributed using a flux-weighted scheme. We found that a flux-weighted method was necessary to select the initial distribution of particles to get an accurate PTRK result.

\begin{tabular}{|l|l|}
\hline \multicolumn{1}{|c|}{ Parameter } & \multicolumn{1}{c|}{ CPU Time (sec) } \\
\hline \hline PTRK (10,201 node problem) & 28 \\
\hline CDR (10,201 node problem) & 524 \\
\hline RST (2 streamlines) & 47 \\
\hline
\end{tabular}

Table 4-2. Comparison of CPU times between CDR and RST for $2 \mathrm{D}$ vertical layer case

For the two vertical layer problem above, and the given parameters, RST, CDR and PTRK matched closely since the timescale for transverse dispersion was much smaller than the transport timescale. To examine transverse dispersion quantitatively, we define the transverse mixing time to be:

$$
\tau_{m}=\frac{w^{2}}{D_{T}}
$$

where $w$ is the width of the layer and $D_{T}$ is the average transverse dispersion. In order to study the effect of transverse dispersion, we increase the number of layers in the domain thereby reducing the length scale for transverse dispersion (see Figure 4-3) Since RST neglects transverse disper- 


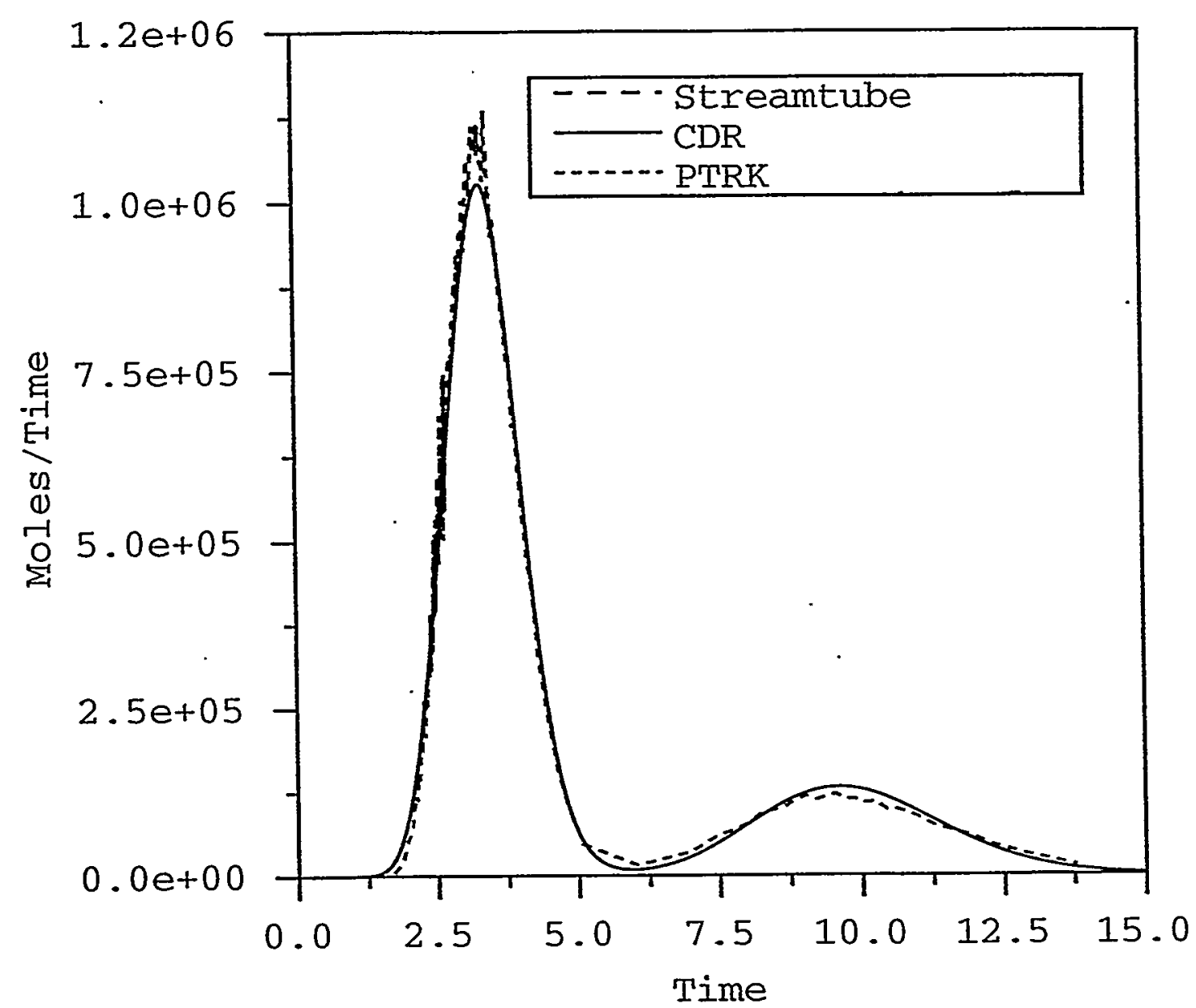

Figure 4-2. Comparison of CDR, PTRK, and RST for transport of nonreactive transport through two layers with differing permeabilities.

sion, the RST results are unaffected by reducing the length scale of transverse dispersion. Figure 4-4 shows how the CDR model breakthrough curves are affected as the length scale of transverse dispersion is reduced. Reducing the length scale allows for transverse dispersion to occur before tracer reaches the outflow plane. If $\tau_{m} \gg \tau$ where $\tau$ is the mean travel time, RST is accurate since transverse dispersion is not important in the system. For this test problem $\tau=6.6$ days. Table 4- 


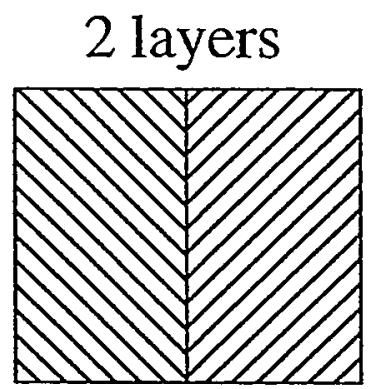

Outflow Plane
4 layers

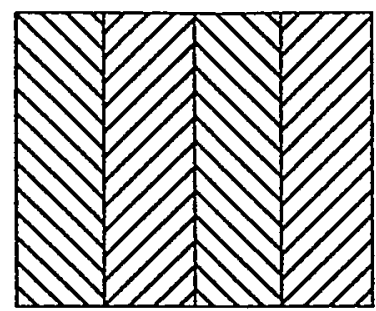

Outflow Plane

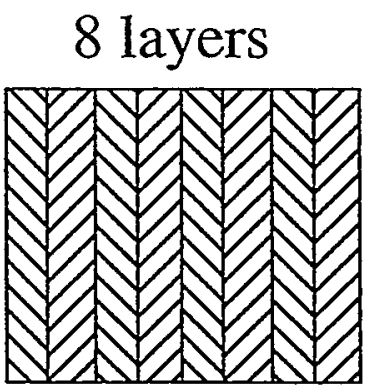

Outflow Plane

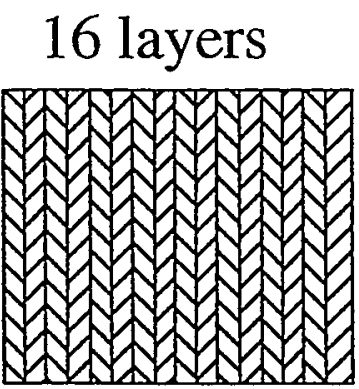

Outflow Plane

Figure 4-3. Vertical layer problem with different numbers of layers. Each layer is $5 \mathrm{~m}$ wide for 2 layer case, $2.5 \mathrm{~m}$ for 4 layer case, $1.25 \mathrm{~m}$ for 8 layer case, $0.625 \mathrm{~m}$ for 16 layer case.

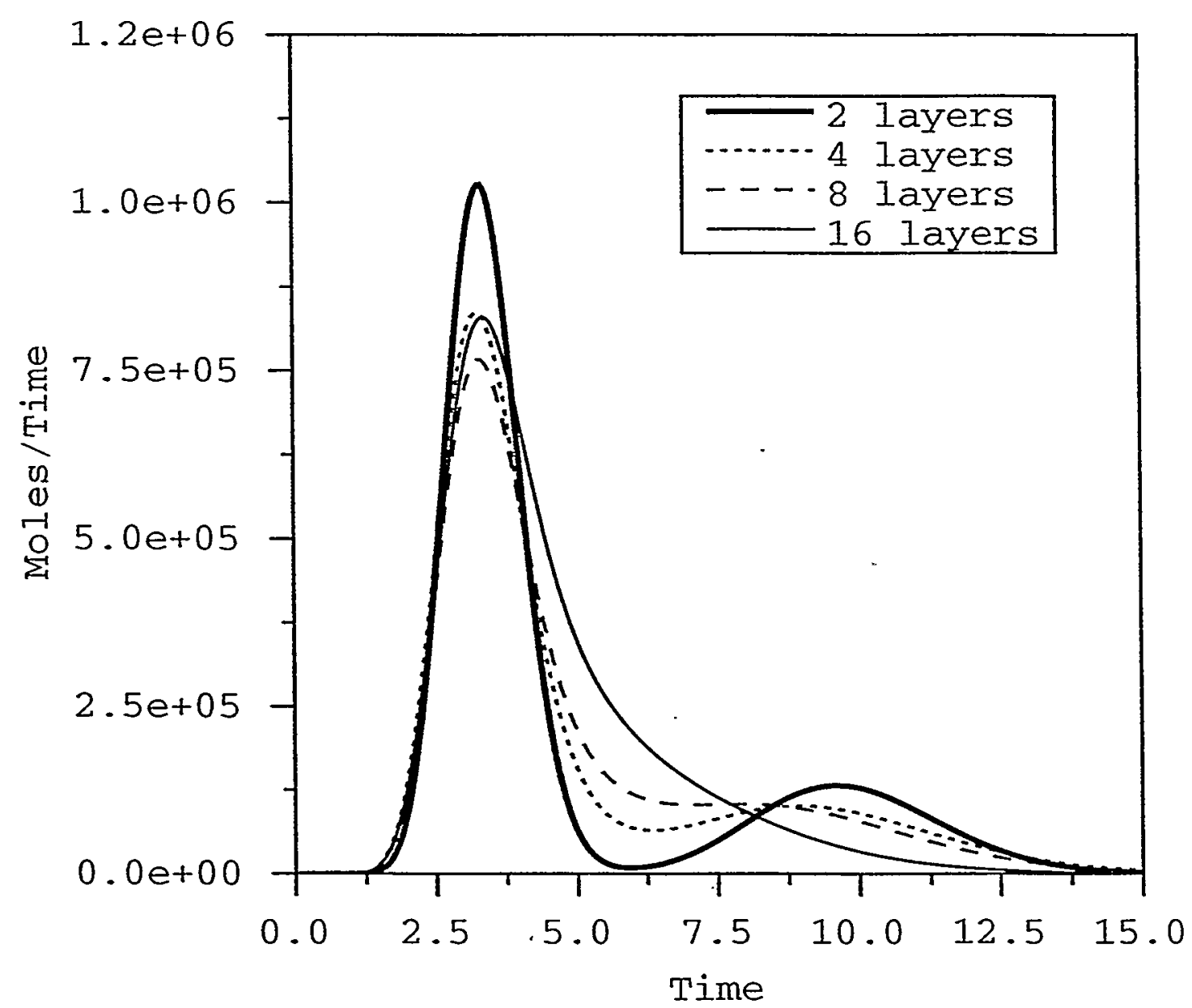

Figure 4-4. CDR model results that illustrate the effect of reducing the length scale for transverse dispersion. Note that the RST result is always equivalent to the 2 layer case since the model neglects transverse dispersion. 
3 explains the results of Figure 4-4. The transverse mixing time for the 2 layer case is greater than the mean travel time of 6.6 days. For the 4,8 and 16 layer cases, $\tau_{m}$ is fast enough for transverse dispersion to have an effect on the breakthrough times. The conclusion from this simple example is that RST will be an accurate method as long as the discrepancy in travel times between adjacent streamlines is not too large.

\begin{tabular}{|l|l|l|}
\hline \multicolumn{1}{|c|}{ Number of Layers } & Width of Layer $(\mathrm{m})$ & $\tau_{\mathrm{m}}$ (days) \\
\hline \hline 2 & 5 & 40.2 \\
\hline 4 & 2.5 & 10.05 \\
\hline 8 & 1.25 & 2.51 \\
\hline 16 & 0.625 & 0.628 \\
\hline
\end{tabular}

Table 4-3. Transverse mixing times

\subsubsection{D Bypass Problem}

For this test case, a $5 \mathrm{~m} \times 5 \mathrm{~m}$ low permeability region is inserted into the model to create a bypass of flow around this region (Figure 4-5). Tables $4-4$ and 4-5 show the parameters used in

\begin{tabular}{|l|l|}
\hline \multicolumn{1}{|c|}{ Parameter } & \multicolumn{1}{c|}{ Value } \\
\hline \hline Low permeability region, $\mathrm{k}_{\text {low }}$ & $5 \times 10^{-17} \mathrm{~m}^{2}$ \\
\hline High permeability region, $\mathrm{k}_{\text {high }}$ & $1 \times 10^{-16} \mathrm{~m}^{2}$ \\
\hline Time of pulse input at top boundary & 1000 days \\
\hline Simulation time & $6 \times 10^{4}$ days \\
\hline molecular diffusion & $1 \times 10^{-10} \mathrm{~m}^{2} / \mathrm{s}$ \\
\hline
\end{tabular}

Table 4-4. Parameters for two-dimensional flow bypass problem 


\begin{tabular}{|l|l|l|l|}
\hline Case No. & \multicolumn{1}{|c|}{ Description } & $\begin{array}{l}\text { Longitudinal } \\
\text { Dispersivity }\end{array}$ & $\begin{array}{l}\text { Transverse } \\
\text { Dispersivity }\end{array}$ \\
\hline \hline a & $\begin{array}{l}\text { PTRK with no longitudinal } \\
\text { dispersion }\end{array}$ & - & - \\
\hline $\mathrm{b}$ & PTRK with longitudinal dispersion & $0.1 \mathrm{~m}$ & - \\
\hline $\mathrm{c}$ & RST (20 streamlines) & $0.1 \mathrm{~m}$ & - \\
\hline $\mathrm{d}$ & $\begin{array}{l}\text { CDR with lower transverse } \\
\text { dispersion }\end{array}$ & $0.1 \mathrm{~m}$ & $0.01 \mathrm{~m}$ \\
\hline $\mathrm{e}$ & $\begin{array}{l}\text { CDR with higher transverse } \\
\text { dispersion }\end{array}$ & $0.1 \mathrm{~m}$ & $0.1 \mathrm{~m}$ \\
\hline
\end{tabular}

Table 4-5. Test cases for two-dimensional flow bypass problem

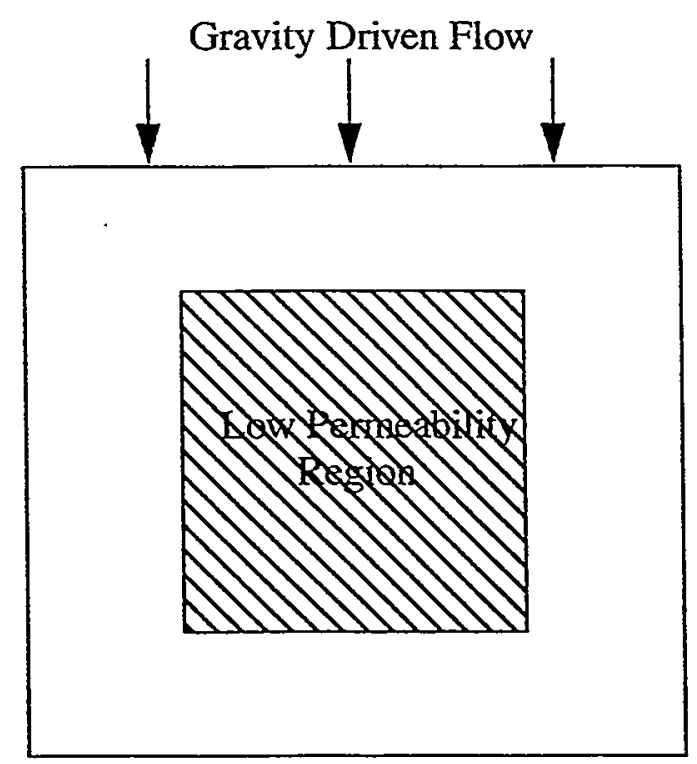

Outflow Plane

Figure 4-5. Two-dimensional flow bypass problem.

this example and the different cases that were simulated. Figure 4-6 shows the streamline pattern in which flows preferentially bypasses the low permeability block.

Figures 4-7 and 4-8 shows the flux averaged breakthrough curves from the bottom of the model for the five cases. Each case results in a breakthrough curve with two distinct peaks. The first peak represents the tracer that completely bypasses the low permeability region. The second peak is obtained from tracer which travels through the low permeability region and is therefore delayed. 


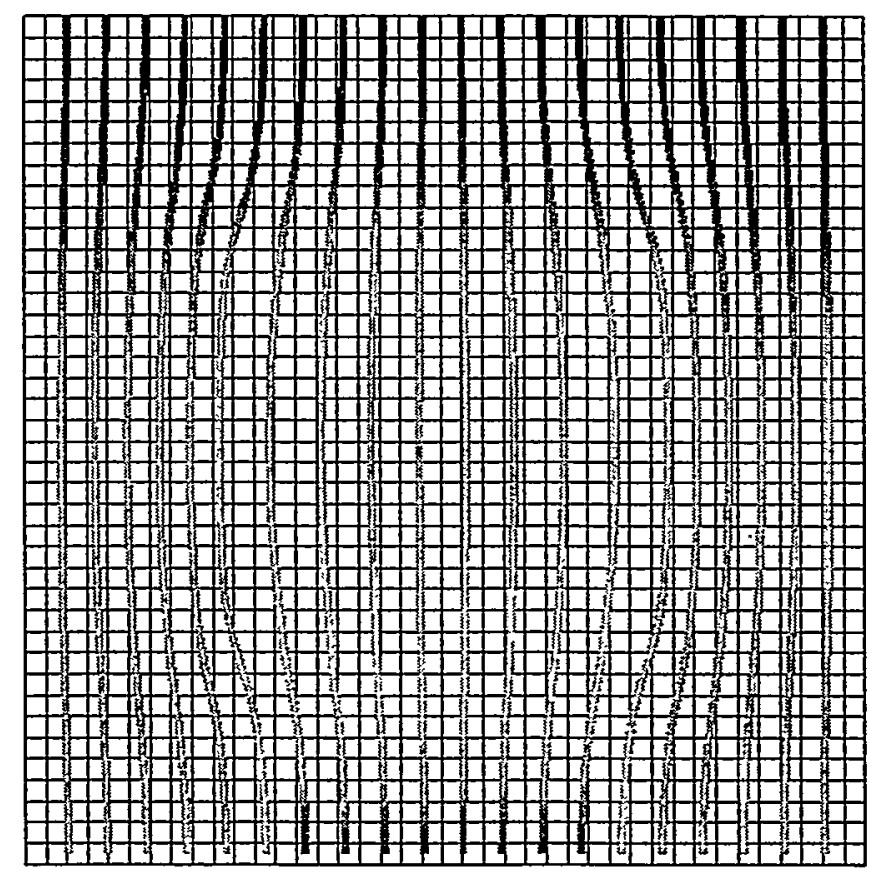

Figure 4-6. Twenty streamlines used by RST for two-dimensional bypass problem.

Figure 4-7 shows that the particle tracking with longitudinal dispersion, the RST method, and the CDR with longitudinal dispersion and low transverse dispersion agree closely. A maximum time step of 900 days and a grid spacing of $0.1 \mathrm{~m}$ is used for the CDR simulation. Figure 4-8 shows the expected result that RST deviates from the CDR solution with high transverse dispersion and the PTRK solution which has no dispersion. The particle tracking simulation with no dispersion shows two distinct peaks. The slight spreading witnessed for this case can be attributed to the nonuniform flow distribution. As the transverse dispersion is increased for the CDR model, the two peaks become less distinct and the CDR model no longer matches the RST model.

Table 4-6 compares the CPU times between the CDR and RST methods. As expected, the PTRK method results in the fastest solutions. RST is only slightly faster than the CDR method. We will see that the computational advantage of RST is more pronounced for the threedimensional verification problems since they contain more spatial nodes resulting in significantly 


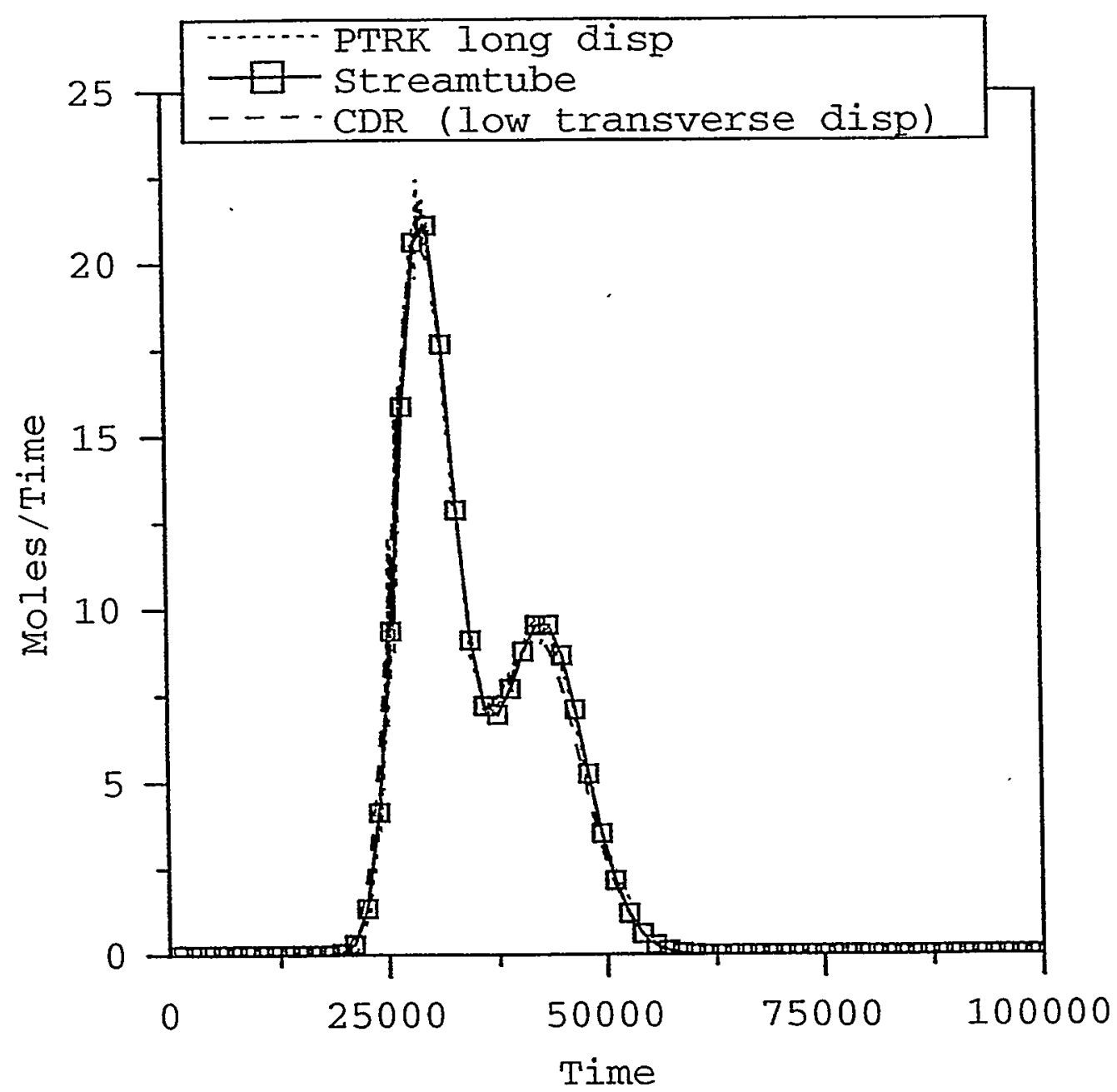

Figure 4-7. Flux averaged breakthrough curves comparing RST (case c), CDR with low transverse dispersion (case d), and PTRK with longitudinal dispersion but no transverse dispersion (case b). 


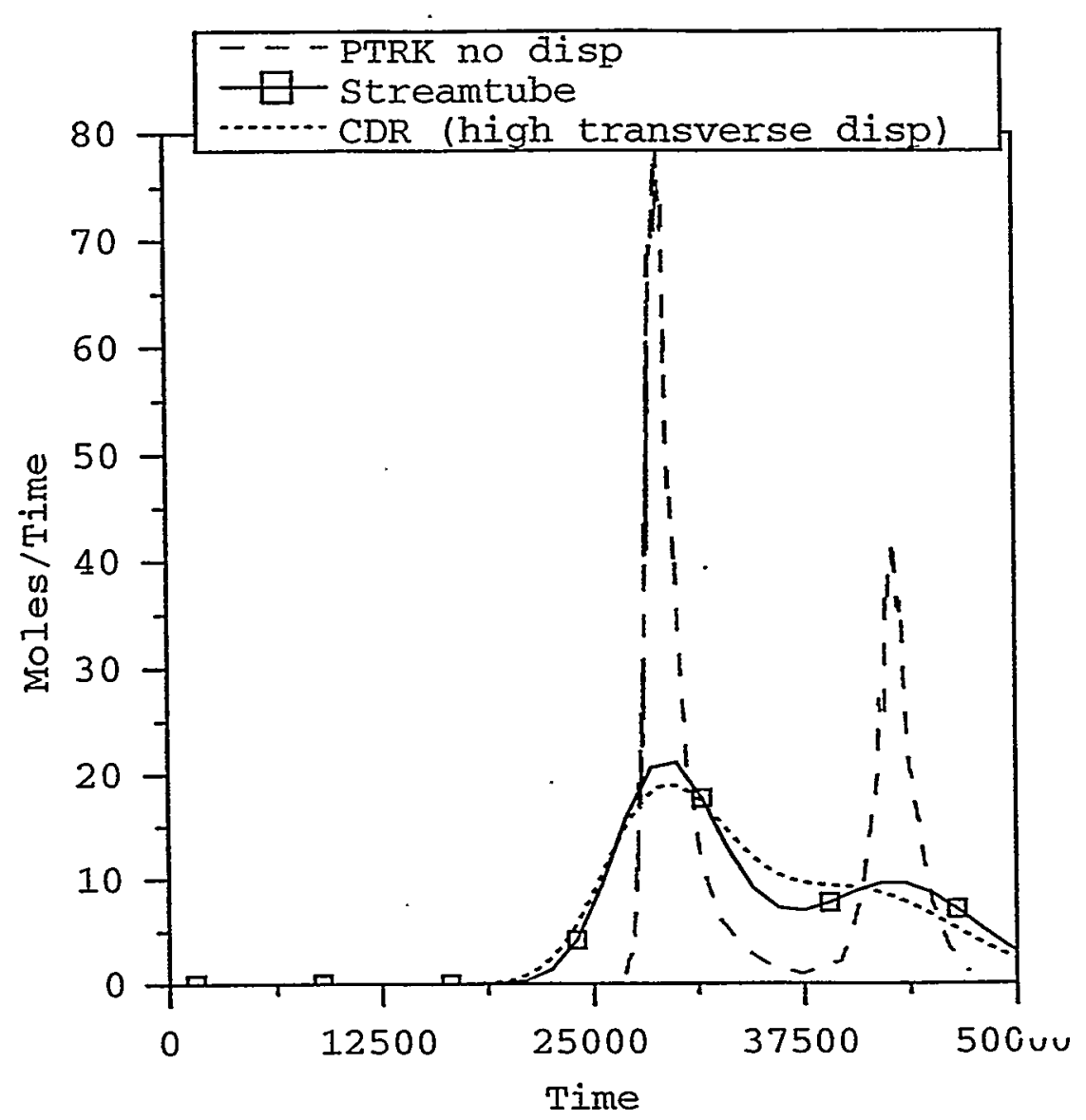

Figure 4-8. Flux averaged breakthrough curves comparing RST (case c), CDR with high transverse dispersion (case e), and PTRK with no dispersion (case a).

slower CDR model runs.We will also see in later sections that RST is more computationally efficient for multicomponent reactive transport problems.

\begin{tabular}{|l|l|l|}
\hline Case No. & \multicolumn{1}{|c|}{ Parameter } & \multicolumn{1}{|c|}{ CPU Time (sec) } \\
\hline \hline a & PTRK with no longitudinal dispersion & 54 \\
\hline b & PTRK with longitudinal dispersion & 103 \\
\hline c & RST with 20 streamtubes & 458 \\
\hline d, e & $\begin{array}{l}\text { CDR with low transverse dispersion (10,201 } \\
\text { node problem) }\end{array}$ & 583 \\
\hline
\end{tabular}

Table 4-6. Comparison of CPU times between CDR and RST for 2D bypass problem 


\subsubsection{D Random Field Problem}

In the next example, we compare CDR, RST and PTRK for a $10 \mathrm{~m}$ by $10 \mathrm{~m}$ random field of permeability. Tracer migration through a random permeability field is more representative of the types of problems for which we will want to utilize RST. A log-normal distribution of permeability was generated using the turning bands algorithm described by Tompson et al (1989). Table 4-7 provides the parameters used in this example.A CDR time step of 900 days and a grid spacing of $0.1 \mathrm{~m}$ is used for this problem. Figure 4-9 shows the twenty streamlines used for this simulation. The individual breakthrough curves from the streamtubes that make up the RST solution are shown in Figure 4-10. Due to the heterogeneous permeability field, each individual streamtube breaks through at a different time. The RST result is a flux weighted average of these individual breakthrough curves. Figure 4-11 shows the flux averaged breakthrough curve from the bottom of the model. The CDR, RST and PTRK models all agree closely for this problem.

\begin{tabular}{|l|l|}
\hline \multicolumn{1}{|c|}{ Parameter } & \multicolumn{1}{c|}{ Value } \\
\hline \hline Geometric Mean Permeability, $\mathrm{k}_{\mathrm{G}}$ & $1 \times 10^{-16} \mathrm{~m}^{2}$ \\
\hline $\ln (\mathrm{k})$ Variance, $\sigma^{2}$ & 1.0 \\
\hline Correlation Length (isotropic) & $1 \mathrm{~m}$ \\
\hline Longitudinal dispersivity (for RST and CDR) & $0.1 \mathrm{~m}$ \\
\hline Transverse dispersivity (CDR) & $0.01 \mathrm{~m}$ \\
\hline Molecular diffusion coefficient & $1 \times 10^{-10} \mathrm{~m}^{2} / \mathrm{s}$ \\
\hline Time of pulse input at top boundary & 1000 days \\
\hline Simulation time & 57000 days \\
\hline
\end{tabular}

Table 4-7. Parameters for $2 \mathrm{D}$ random field problem

Using fewer than twenty streamtubes yields poor results; as can be seen in Figure 4-12. The breakthrough curves computed using five and ten streamtubes do not reproduce the CDR results. For twenty or more streamtubes, the RST result closely approximates the CDR result although even the 20 streamtube case shows some discrepancies. These discrepancies could be due to the 
neglect of transverse dispersion by the RST model. Note that the number of streamtubes necessary to obtain accurate results is strongly dependent on a number of parameters (e.g. size of the domain, correlation length, variance in $\ln (\mathrm{K})$, etc.). In all of the verification problems we simply determine

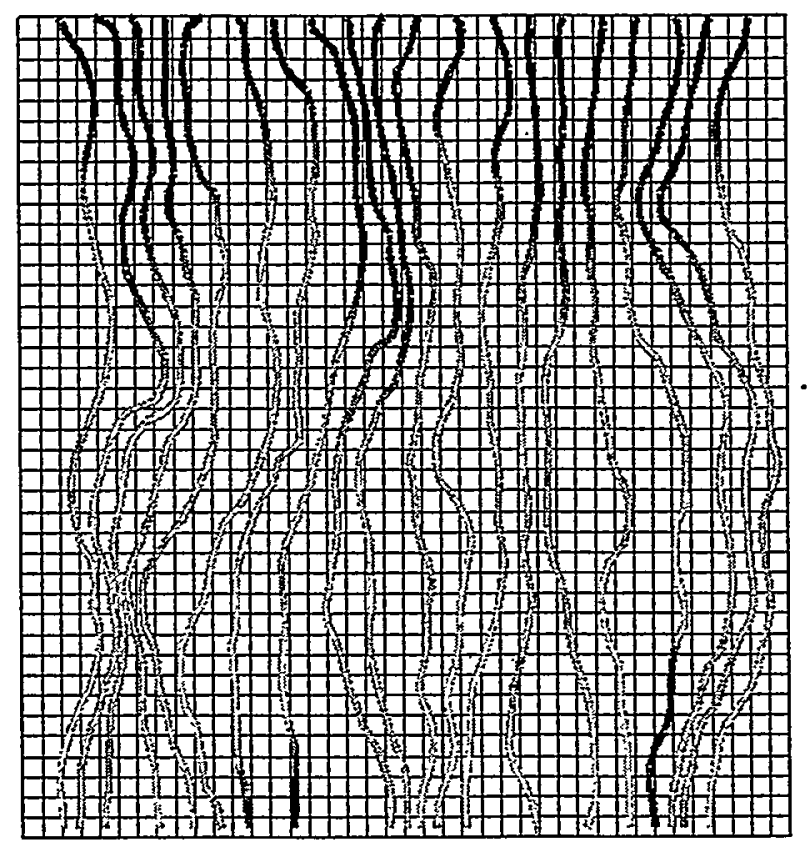

Figure 4-9. Twenty streamlines used by RST to simulate nonreactive transport through a log normally distributed field of permeability.

that a given number of streamlines is adequate by performing a trial-and-error sensitivity analysis. After the verification problems we will provide some general guidelines in choosing the number of streamlines necessary to properly characterize the flow field. Table 4-8 compares the CPU times between the CDR and RST methods. As with the two-dimensional bypass problem, RST is only slightly faster than CDR. The methods are comparable in CPU time since 20 one-dimensional 


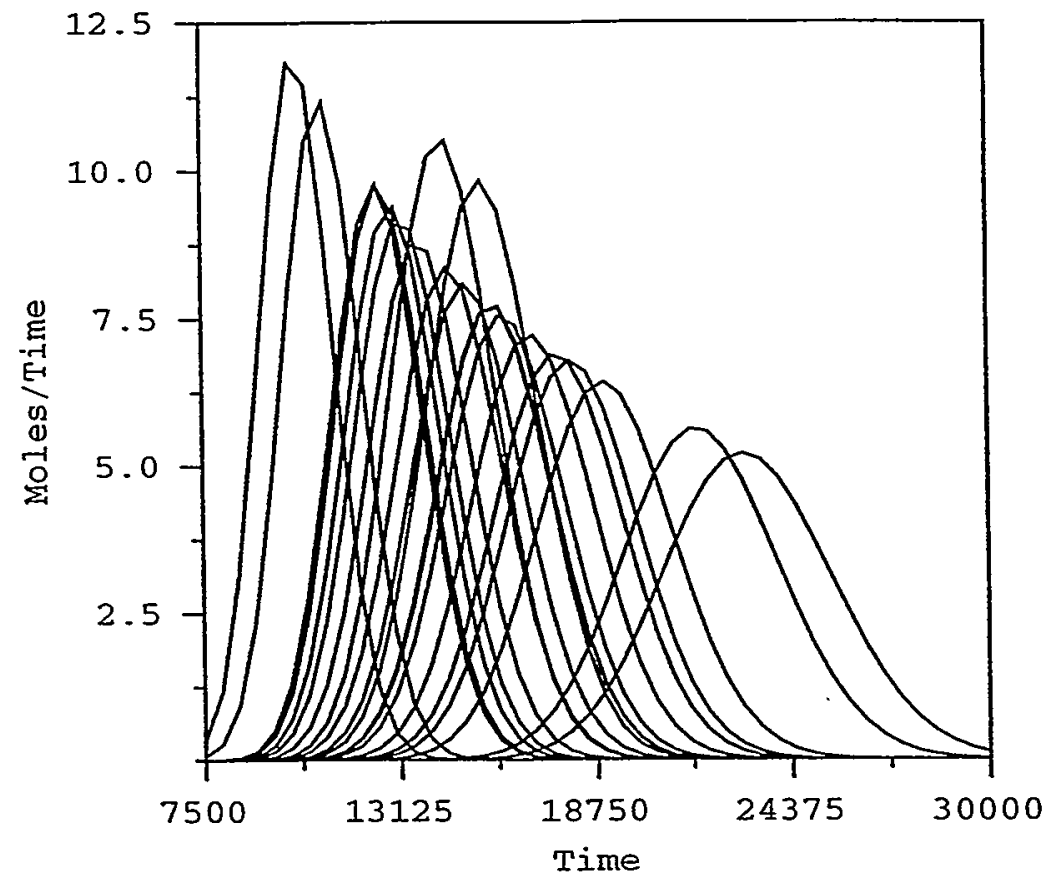

Figure 4-10. Individual breakthrough curves from each streamline. A flux-weighted average of these breakthrough curves is used to obtain the RST solution.

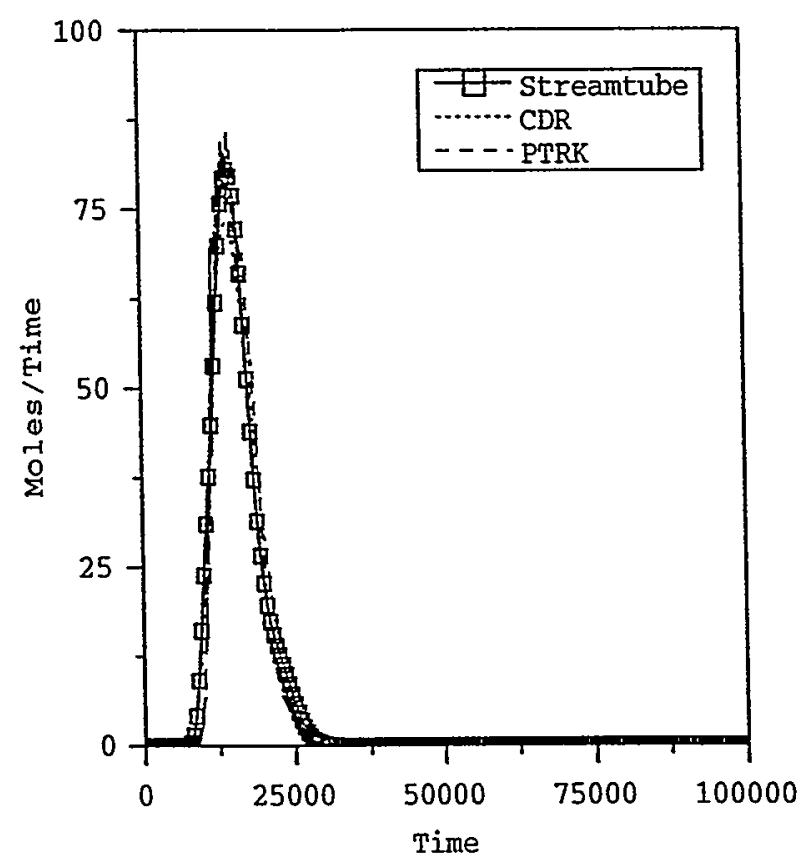

Figure 4-11. Flux averaged breakthrough curves comparing RST, CDR and PTRK for nonreactive transport through a log-normally distributed permeability field. 


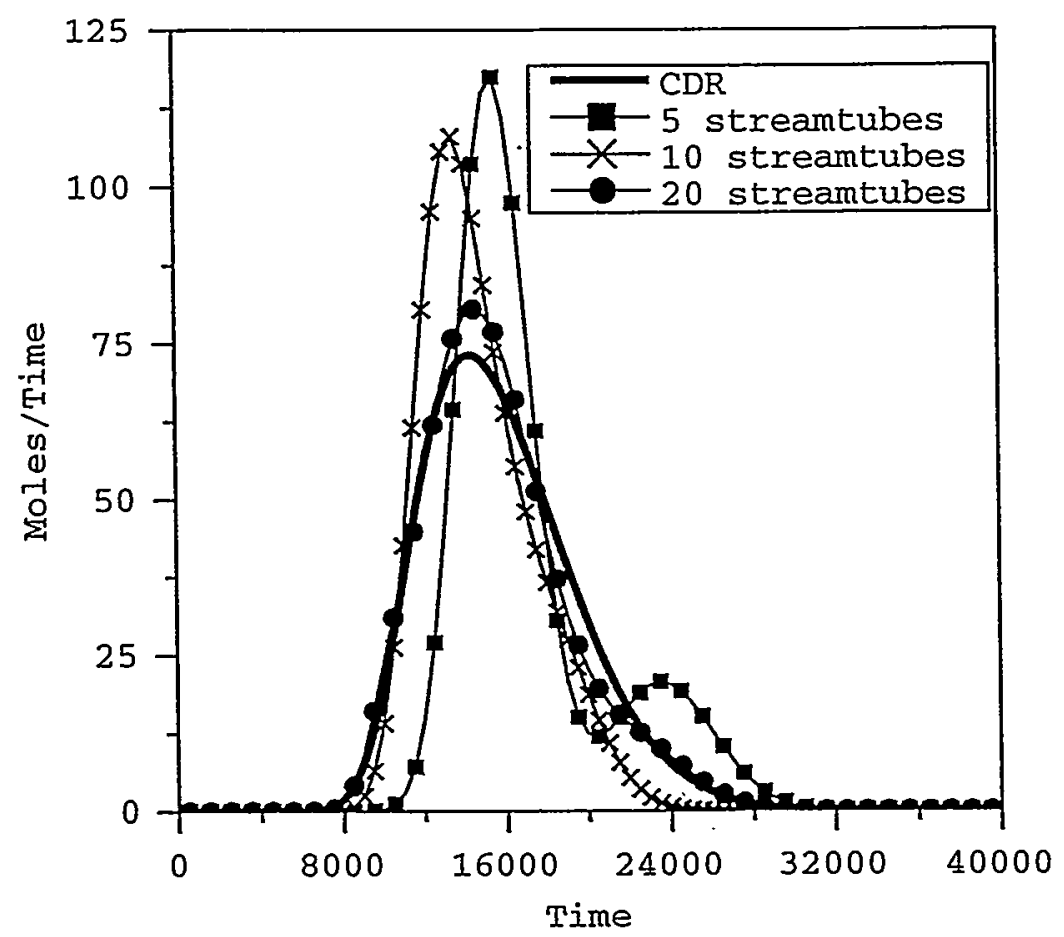

Figure 4-12. The effect of varying the number of streamlines. Flux averaged breakthrough curves of RST using 5, 10, and 20 streamtubes.

simulations (one for each streamline) take almost as long to run as the 10,201 node twodimensional CDR simulation.

\begin{tabular}{|l|l|}
\hline \multicolumn{1}{|c|}{ Parameter } & \multicolumn{1}{c|}{ CPU Time (sec) } \\
\hline \hline CDR (10,201 node problem) & 559 \\
\hline RST (20 streamlines) & 446 \\
\hline PTRK with longitudinal dispersion & 39 \\
\hline
\end{tabular}

Table 4-8. Comparison of CPU times between CDR and RST for 2D bypass problem

A key parameter that could affect the accuracy of RST is the variance of $\ln (k)$. As the variance is increased, the velocity field becomes more heterogeneous and one would expect that RST will require more streamlines to accurately characterize the flow field. Another key question is if any number of streamlines will be sufficient to produce an accurate result for highly heterogeneous flow fields. As flow heterogeneity increases, we expect greater velocity contrast for 
(A) $\ln (\mathrm{K})$ variance $=1.5$ 20 streamlines

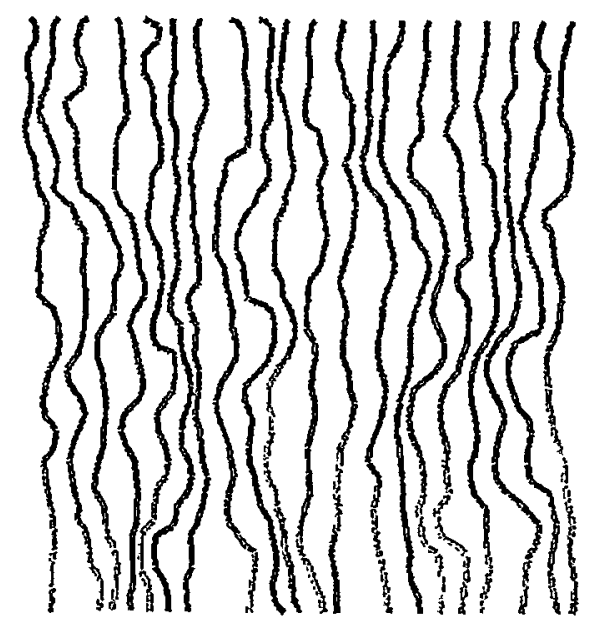

(B) $\ln (\mathrm{K})$ variance $=1.75$

50 streamlines

(C) $\ln (\mathrm{K})$ variance $=2.0$

50 streamlines .
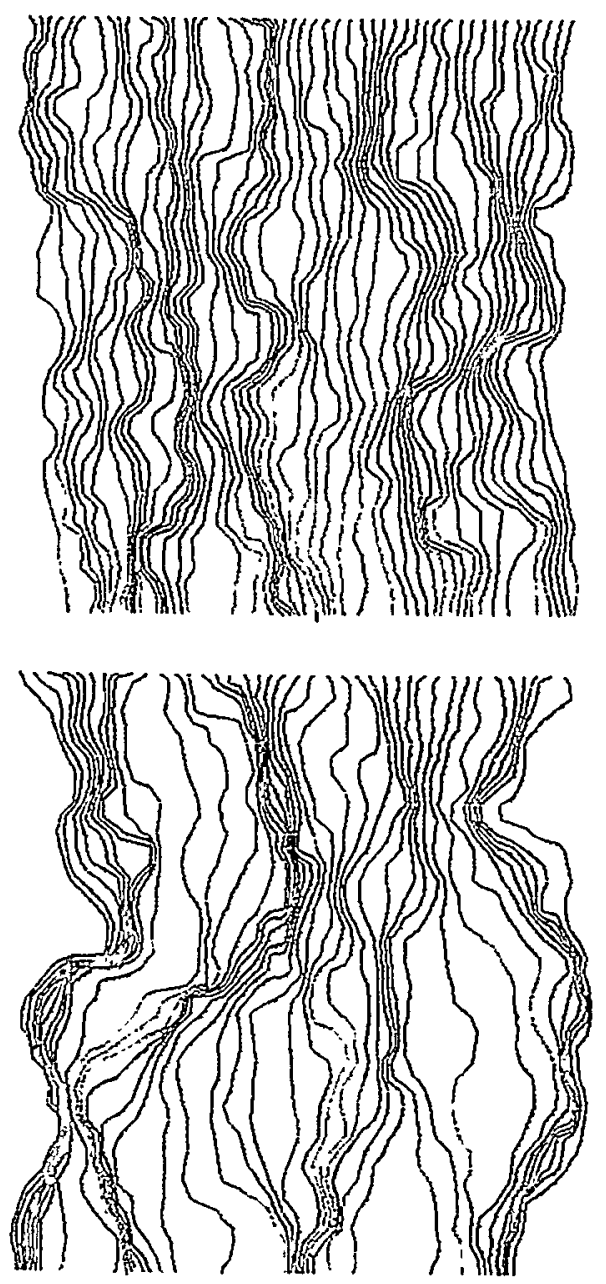

Figure 4-13. RST streamlines for different values of the $\ln (\mathrm{k})$ variance. Blue represents short travel times and red represents long travel times. 


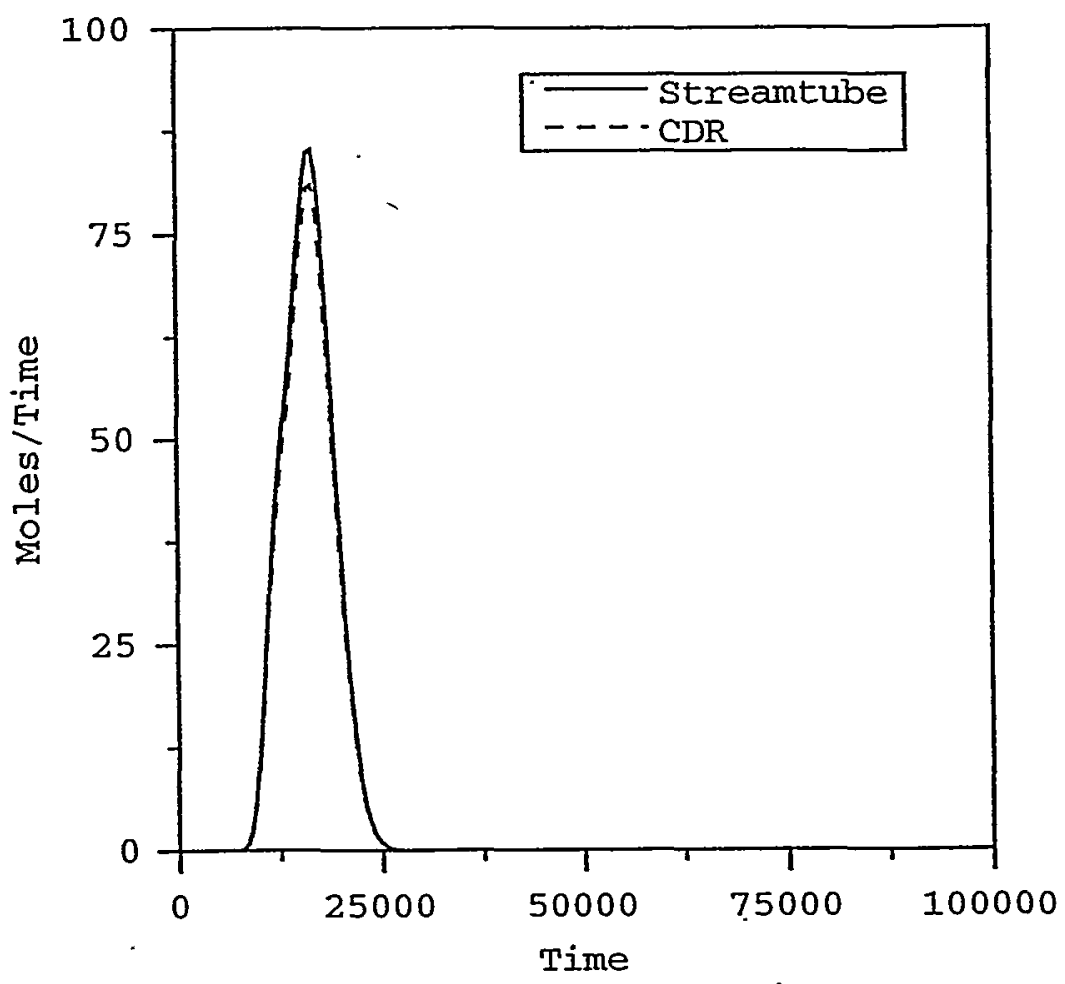

Figure 4-14. Comparison of RST and CDR for a $\ln (k)$ variance of 1.5.

adjacent streamlines, which will lead to increased transverse dispersion, a process that is not included in the RST model. In the next set of simulations we keep all parameters of the random field the same except for the variance which we gradually increase in each simulation.

Figure 4-13 is similar to Figure 4-9 but with increasing variance of $\ln (k)$. Note that blue portions of the streamlines represent short travel times and red represent long travel times on the figure. As the variance increases, the streamlines become highly tortuous. Figure 4-14 compares $\mathrm{CDR}$ and RST for a $\ln (\mathrm{k})$ variance of 1.5 . For this case, the methods still agree very closely. We only required 20 streamlines to obtain the match with the CDR method. Figure 4-15 compares the methods for a $\ln (\mathrm{k})$ variance of 1.75 . For this case, the $C D R$ method exhibits more dispersion than the RST model. Fifty streamlines were required for RST to converge. By converge, we mean that additional streamlines (more than 50) did not affect the RST results. For this problem, transverse mixing between adjacent streamlines may be causing RST to fail. Another possibility could be that 


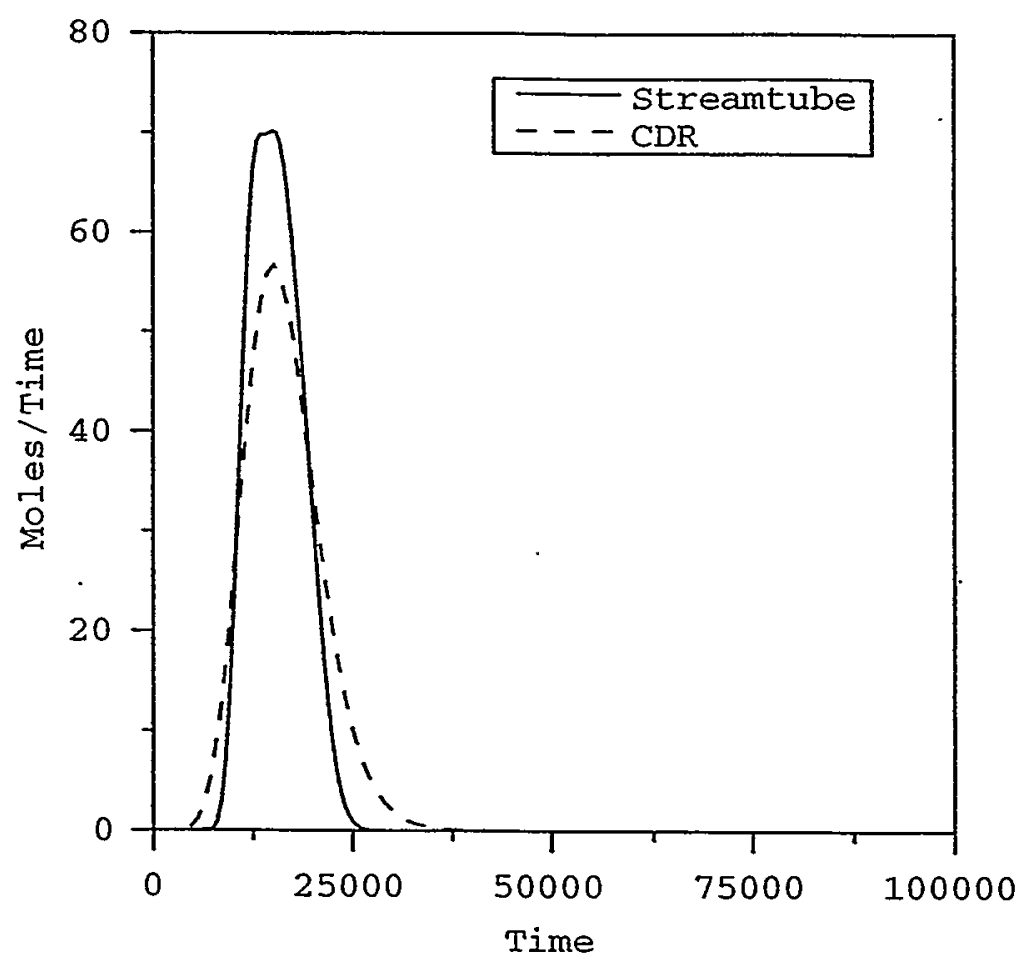

Figure 4-15. Comparison of RST and CDR for a $\ln (k)$ variance of 1.75

for this heterogeneous flow field, the CDR model exhibits numerical dispersion resulting in a more disperse breakthrough curve at the control plane.

Figure 4-16 compares RST and CDR for a $\ln (k)$ variance of 2.0. For this case the methods produce very different results. Again 50 streamlines were required for RST to converge. Although, the difference between the methods is greater, the trends are similar to the $\ln (\mathrm{k})$ variance of 1.75 case. The CDR result is disperse, whereas the RST results produces several sharp peaks. Further analysis will be required to determine the cause of the discrepancies but it is clear that the level heterogeneity in the velocity field is an important factor for the effectiveness of the RST and CDR methods. 


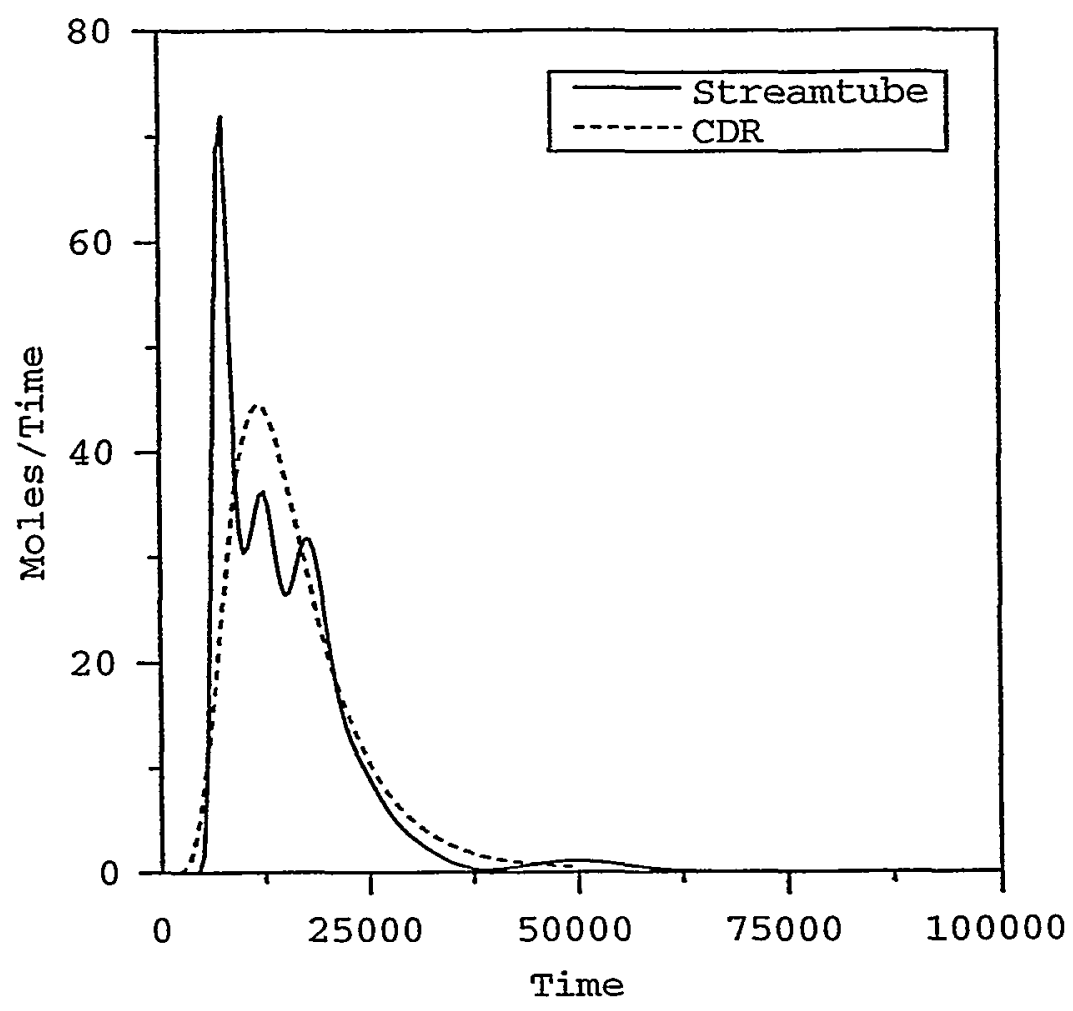

Figure 4-16. Comparison of RST and $C D R$ for a $\ln (k)$ variance of 2.0 .

\subsection{D Nonreactive Transport}

The next three verification problems compare the CDR, PTRK, and RST methods for flow through a three-dimensional block. In all three examples, the problem domain is a cube $10 \mathrm{~m}$ on each side. The problems are similar to the two dimensional ones in which we compare the methods for flow through two vertical layers, a simple bypass scenario, and for a random field. The streamlines are evenly spaced and are started at the center of specified grid blocks at inlet plane. We extract fluxes from the three-dimensional flow simulations and use these fluxes to partition the flow among each of the one-dimensional streamlines. This procedure is discussed in greater detail in Chapter 2.

\subsubsection{D Vertical Layer Problem}

In this example, we simulate flow through two vertical layers with different permeabilities. As with the two-dimensional verification problems, the top and bottom boundaries are assigned 


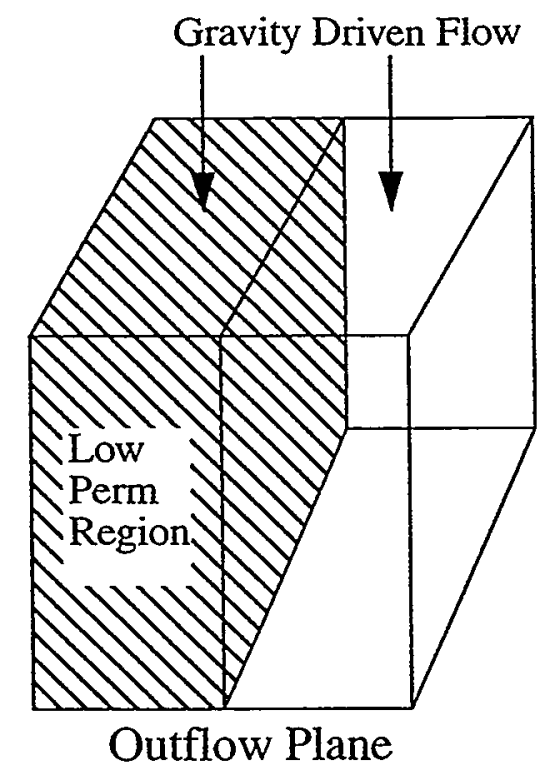

Figure 4-17.Three-dimensional flow problem with vertical layers.

equal pressures resulting in gravity driven flow through the block. This problem is set up with identical properties as the two-dimensional vertical flow problem (Table 4-1). As expected, the CDR, PTRK and RST methods produce similar results (Figure 4-18) since the CDR model was run with a fine grid spacing and timestep. In fact, the results shown in Figure 4-18 and Figure 4-2 are identical. Again, for this simple problem only two streamlines are necessary to replicate the CDR results. Table 4-9 shows the CPU times for the CDR, PTRK and RST methods. RST is far more computationally efficient for this three-dimensional vertical layer problem since the CDR method requires 68,921 nodes whereas RST only has to compute on two one-dimensional grids. 


\begin{tabular}{|l|l|}
\hline \multicolumn{1}{|c|}{ Parameter } & \multicolumn{1}{c|}{ CPU Time (sec) } \\
\hline \hline CDR (68,921 node problem) & 2953 \\
\hline PTRK(68,921 node problem) & 60 \\
\hline RST (2 streamlines) & 14 \\
\hline
\end{tabular}

Table 4-9. Comparison of CPU times between CDR and RST for 3D vertical layer problem

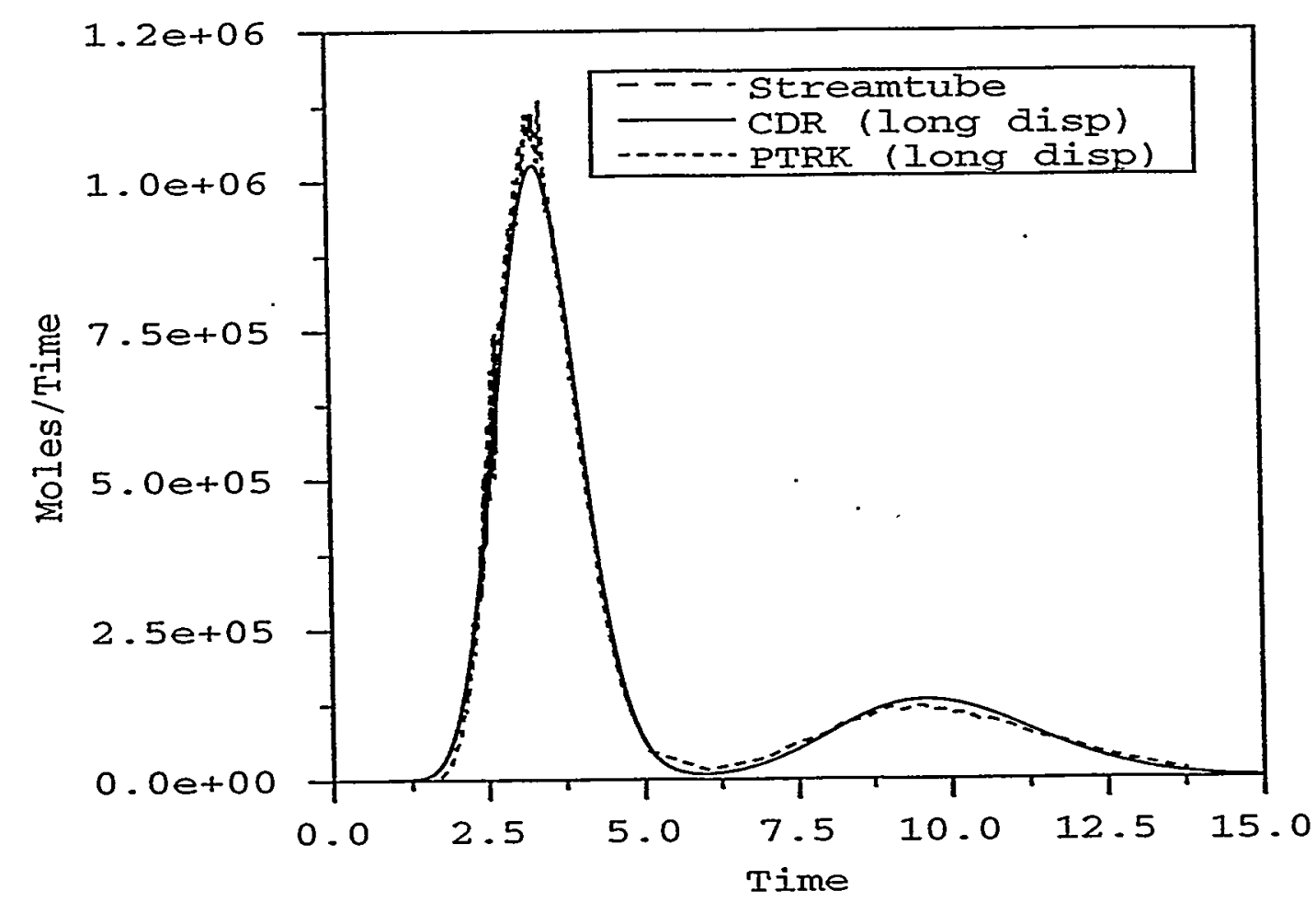

Figure 4-18. Flux averaged breakthrough curves comparing the CDR, PTRK and RST approaches for three-dimensional flow with vertical layers. 


\subsubsection{D Bypass Problem}

For this test case, a $5 \mathrm{~m} \times 5 \mathrm{~m} \times 5 \mathrm{~m}$ low permeability region is inserted into the model to create a bypass of flow around this region (Figure 4-19). Table 4-10 shows the parameters used for

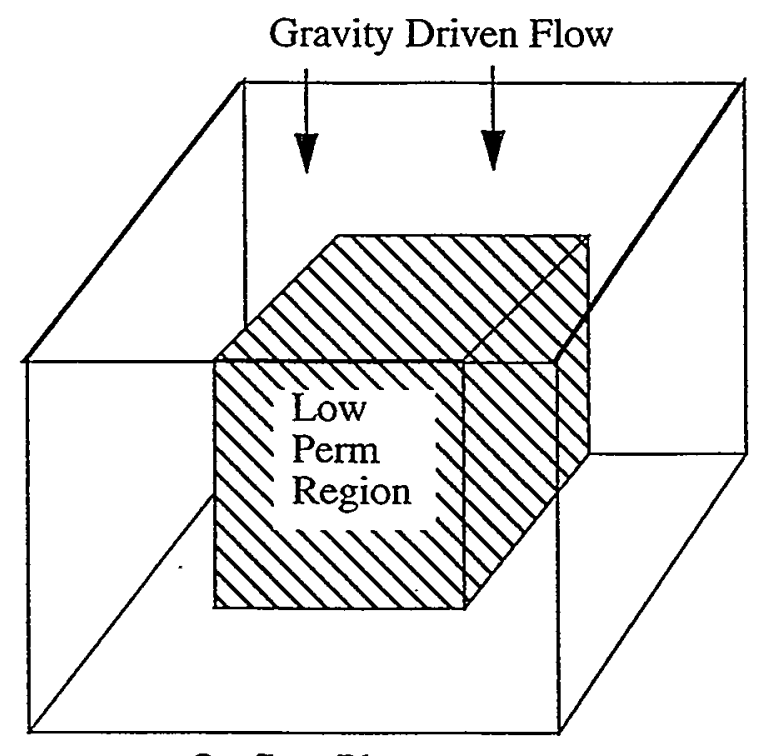

Outflow Plane

Figure 4-19. Three-dimensional flow bypass problem.

this study. We compare RST to PTRK with longitudinal dispersion and in the next example we

\begin{tabular}{|l|l|}
\hline \multicolumn{1}{|c|}{ Parameter } & \multicolumn{1}{c|}{ Value } \\
\hline \hline Low permeability region, $\mathrm{k}_{\text {low }}$ & $5 \times 10^{-14} \mathrm{~m}^{2}$ \\
\hline High permeability region, $\mathrm{k}_{\text {high }}$ & $1 \times 10^{-13} \mathrm{~m}^{2}$ \\
\hline Time of pulse input at top boundary & 1 day \\
\hline Simulation time & 40 days \\
\hline molecular diffusion & $1 \times 10^{-10} \mathrm{~m}^{2} / \mathrm{s}$ \\
\hline grid spacing & $0.1 \mathrm{~m}$ \\
\hline
\end{tabular}

Table 4-10. Parameters for three-dimensional flow bypass problem

compare RST to the CDR model. Figure 4-20 shows the RST and PTRK compare closely. In three dimensions, the bypass does not produce a two-peak breakthrough curve since most of the tracer is able to bypass the low permeability region. In addition, different permeabilities were chosen for 
the low and high permeabilities in the three-dimensional case resulting in much faster breakthrough times. The PTRK breakthrough curve exhibits a slight tail at later times representing the small flux of tracer that interacts with the low permeability unit. This tail is not captured by the RST method when 100 streamlines were used, however using 200 streamlines captures the tail and

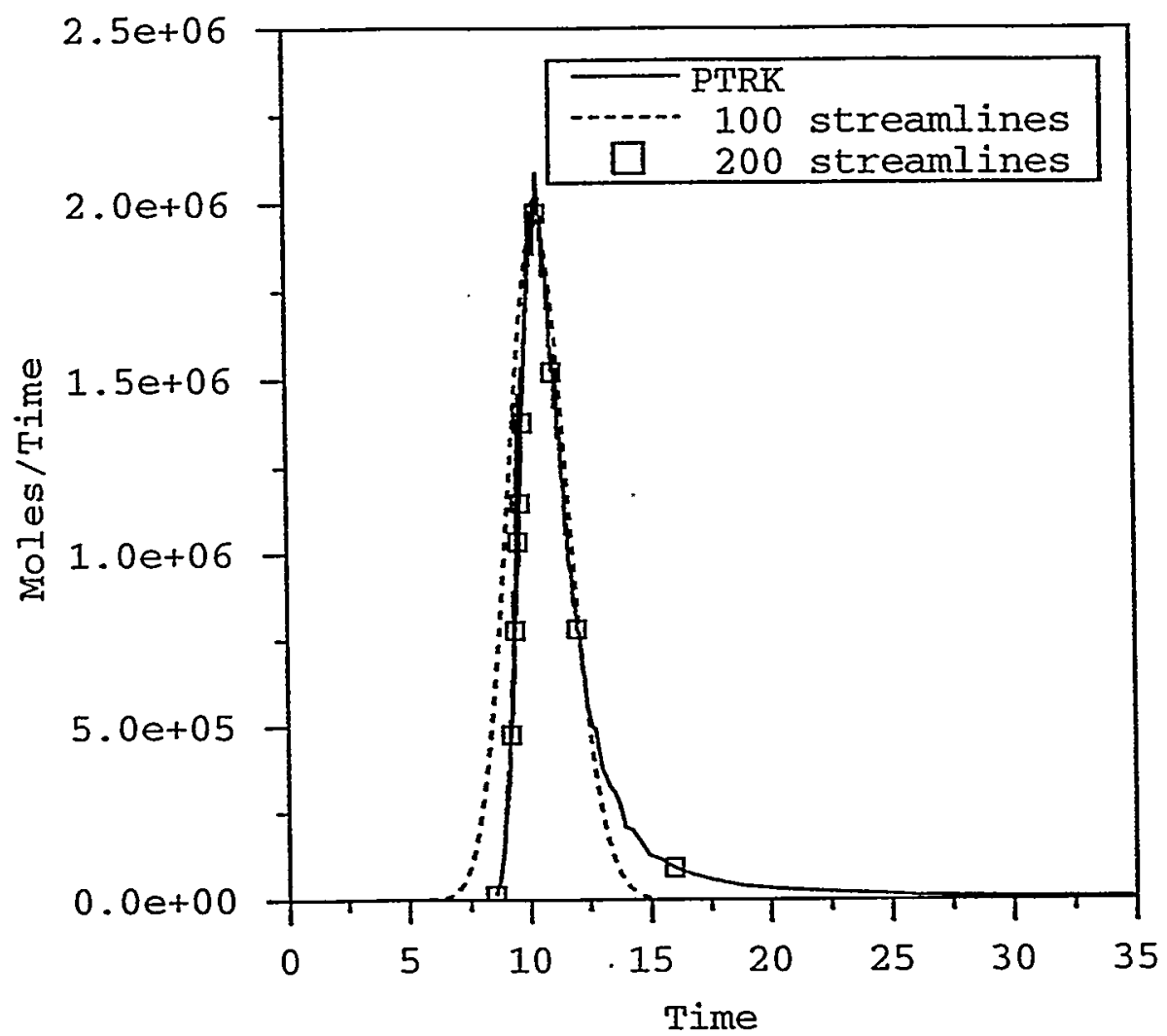

Figure 4-20. Flux averaged breakthrough curves comparing the PTRK and RST methods for three-dimensional bypass problem.

agrees well with the PTRK method. This example illustrates a common error that we encounter with the RST method. We find that the RST method often has trouble capturing tails in breakthrough curves accurately. Tails in the breakthrough curves are often caused by tracer which is delayed due to transverse mixing into low permeability regions. RST. requires numerous streamlines to capture this behavior. The next example also shows that RST overestimates the peak and underestimates the tail relative to the CDR method. 


\subsubsection{D Random Field Problem}

In the next example, we compare RST and CDR for nonreactive transport through a threedimensional random permeability field. This is the only example in which we compare RST to CDR for a three-dimensional problem because, in order to make the comparison, we required a fine grid spacing (132,651 nodes) that results in a computationally intensive CDR simulation. We used 100 streamlines to obtain the RST result. Table 4-10 shows the parameters used for this simulation.

Figure 4-21 shows that both methods exhibit a double peak behavior and agree closely. The

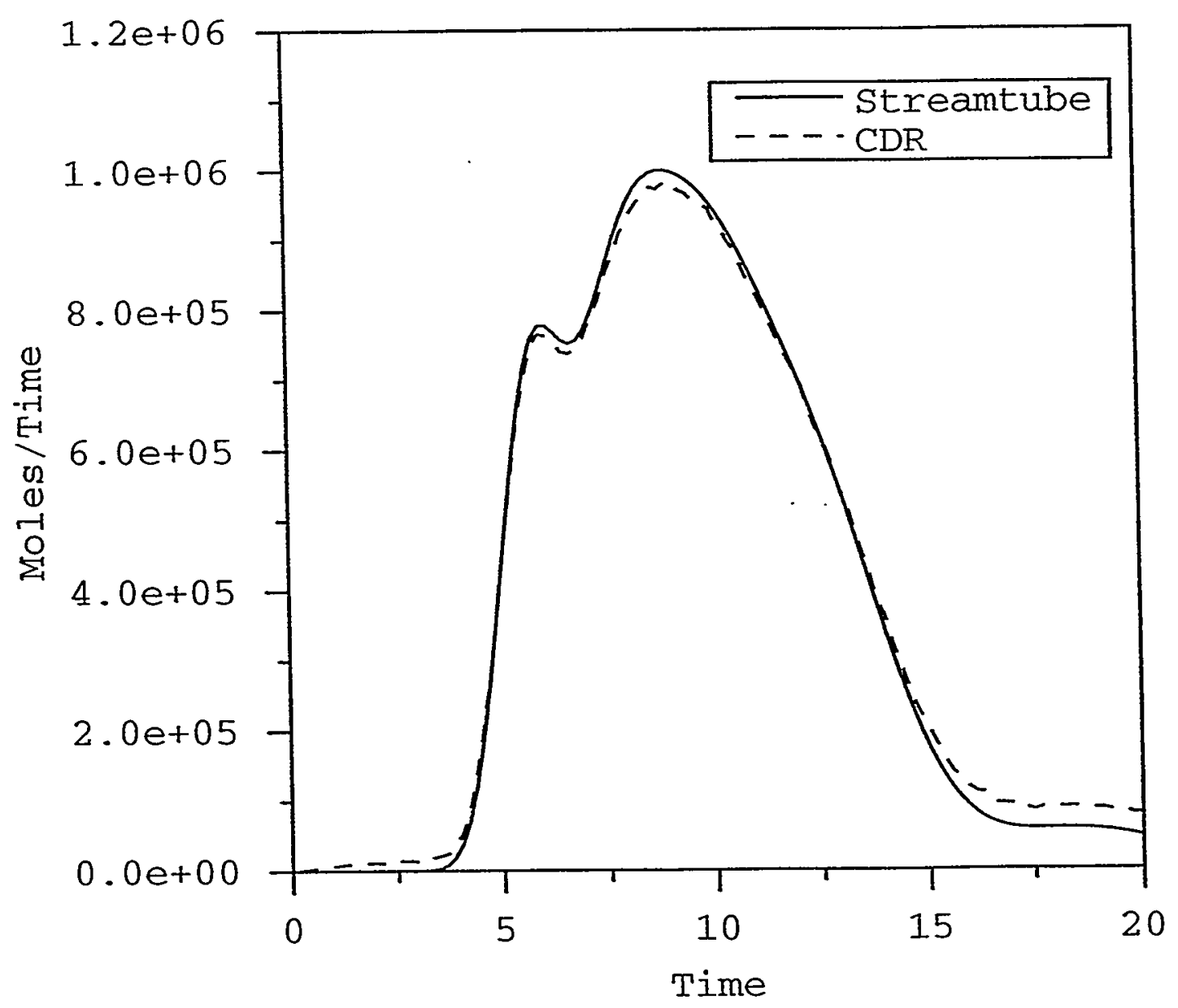

Figure 4-21. Flux averaged breakthrough curves comparing the PTRK and RST methods for three-dimensional random field problem. 
discrepancies between the methods are similar to the previous example. The RST model slightly over predicts the first peak and underestimates the tail. These examples show that RST tends to

\begin{tabular}{|l|l|}
\hline \multicolumn{1}{|c|}{ Parameter } & \multicolumn{1}{c|}{ Value } \\
\hline \hline Simulation Time & 20.0 days \\
\hline Time step & 0.05 days \\
\hline Grid spacing & $0.2 \mathrm{~m}$ \\
\hline Geometric Mean Permeability & $1 \times 10^{-12} \mathrm{~m}^{2}$ \\
\hline In(k) Variance & 1.0 \\
\hline Correlation Length (isotropic) & $1 \mathrm{~m}$ \\
\hline Longitudinal dispersivity (for RST and CDR) & $0.1 \mathrm{~m}$ \\
\hline Transverse dispersivity (for CDR) & 0.01 \\
\hline Molecular diffusion coefficient & $1 \times 10^{-10} \mathrm{~m}^{2} / \mathrm{s}$ \\
\hline Simulation time & 20 days \\
\hline Time of pulse input at top boundary & 1 day \\
\hline
\end{tabular}

Table 4-11. Parameters for three-dimensional random field problem

underestimate the spreading and overestimate the peak concentration. For Performance Assessment models, the possible numerical error incurred by RST is conservative. If a fine enough grid spacing is not used with CDR, the model tends to exhibit too much spreading due to numerical dispersion which underestimates the peak concentration resulting in a nonconservative prediction. Table 4-12. compares the CPU times for the RST and CDR methods. The table illustrates that for

\begin{tabular}{|l|l|}
\hline \multicolumn{1}{|c|}{ Parameter } & \multicolumn{1}{c|}{ CPU Time (sec) } \\
\hline \hline CDR (132,651 node problem) & 10740 \\
\hline RST (100 streamlines) & 300 \\
\hline
\end{tabular}

Table 4-12. Comparison of CPU times between CDR and RST for 3D vertical layer problem

three-dimensional problems, the computational savings using RST are greater than the two- 
dimensional examples.Speifically, RST is about 35 times faster than CDR for this example, wherase it was only about 3 times faster for the two-dimensional simulations.

\subsection{D Transport with Linear Retardation}

For the next set of test problems, we include a linear retardation factor to simulate the sorption of a contaminant. Smith et al. (1995) and Tompson et al. (1996) showed that chemical reactivity and permeability are often inversely related and that this relationship suggests that significant contaminant transport will occur in zones of minimal reactivity. The key new feature in these problems is that they contain correlated chemical and permeability heterogeneity. Specifically, each streamline will encounter a different amount of retardation since the distribution coefficient, $\mathrm{K}_{\mathrm{d}}$, varies along each streamline and also among streamlines. Recall that we extract properties such as $\mathrm{K}_{\mathrm{d}}$ from the Pollock (1988) method for the two- or three-dimensional flow simulation in which a $\mathrm{K}_{\mathrm{d}}$ is known at each spatial node (Figure 2-4).

\subsubsection{D Bypass Problem}

In this example, we redo the $2 \mathrm{D}$ bypass problem with linear retardation. We assume that the $5 \mathrm{~m}$ by $5 \mathrm{~m}$ low permeability region (Figure 4-5) sorbs contaminant with a $\mathrm{K}_{\mathrm{d}}$ of $0.5 \mathrm{cc} / \mathrm{g}\left(\mathrm{R}_{\mathrm{f}}=5.6\right)$, whereas the high permeability region does not sorb. All other parameters are identical to Table 44. Figure 4-22 shows flux averaged breakthrough curves comparing RST and CDR. The figure shows that there are discrepancies between the methods for this test problem. CDR and RST match for the first peak which represents the tracer that migrated through the non-sorbing, high permeability zone. The methods produce different results for the second peak. In fact for the tracer that sorbs, RST produces two delayed peaks, one at $7 \times 10^{4}$ days and one at $1.6 \times 10^{5}$ days, whereas CDR produces one dispersed peak at $1.6 \times 10^{5}$ days. The reason for the discrepancy is related to the $\mathrm{K}_{\mathrm{d}}$ 's that are extracted from the two-dimensional flow simulation. Recall that our method provides the 


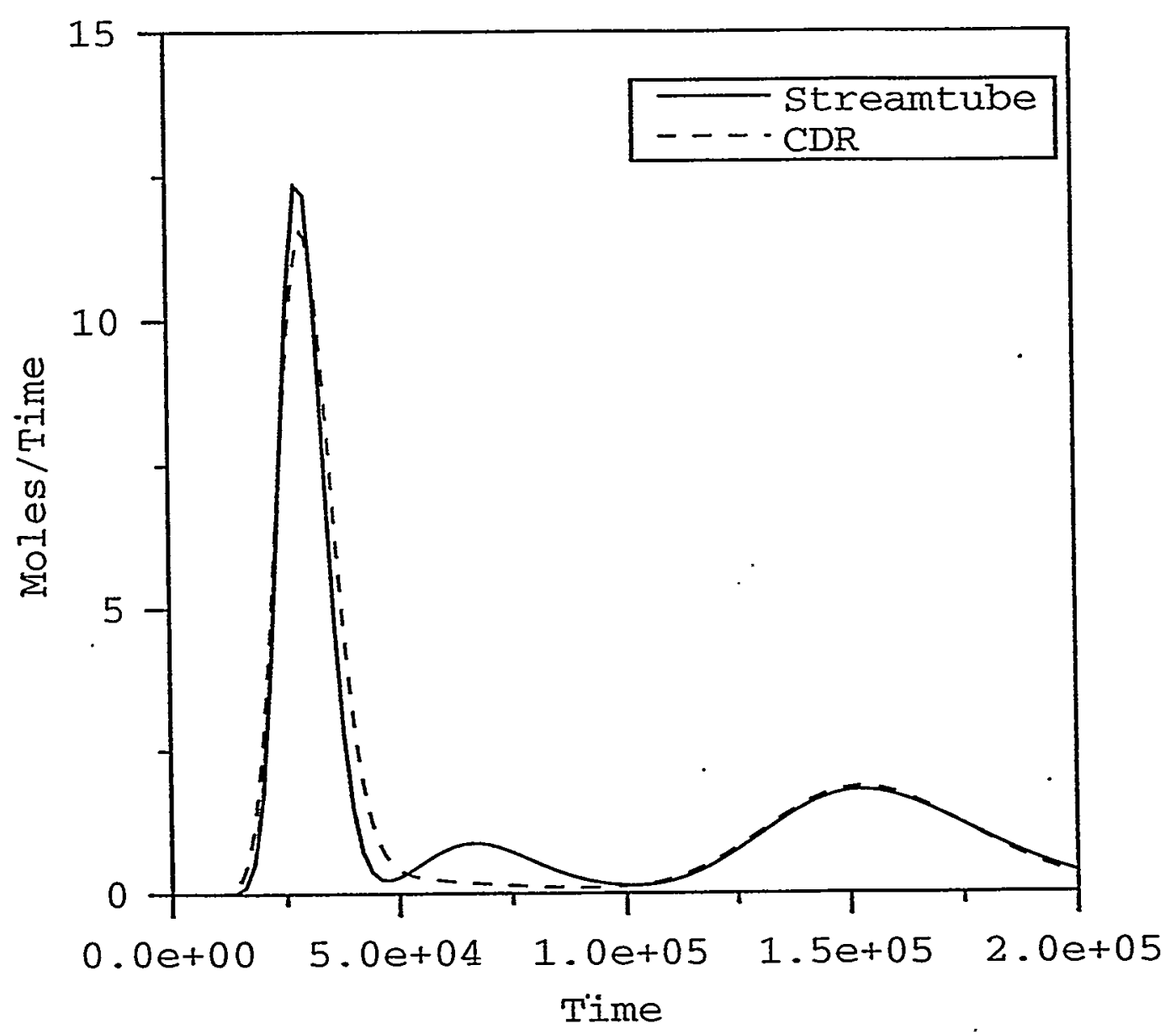

Figure 4-22. Flux averaged breakthrough curves comparing RST and CDR for two-dimensional flow bypass problem with linear retardation in the high permeability layer. Twenty streamlines are used for this simulation.

distance along each streamline, and the travel times at arbritrary positions along the streamline. For each position along the streamline, our method extracts the $K_{d}$ value from the nearest node in the two-dimensional simulation. In this example, we have sharp interfaces between sorbing and nonsorbing regions. Therefore, extracting the nearest node results in the inaccuracy of RST for this 
problem due to the sharp interface between sorbing and nonsorbing regions. Figure 4-23 shows that the problem is remedied by using more streamlines. This example illustrates that adding heterogeneity in the chemical reactivity can cause RST to require more streamlines (50 in this case) for an accurate solution. The nonreactive version of this problem described in section only required 20

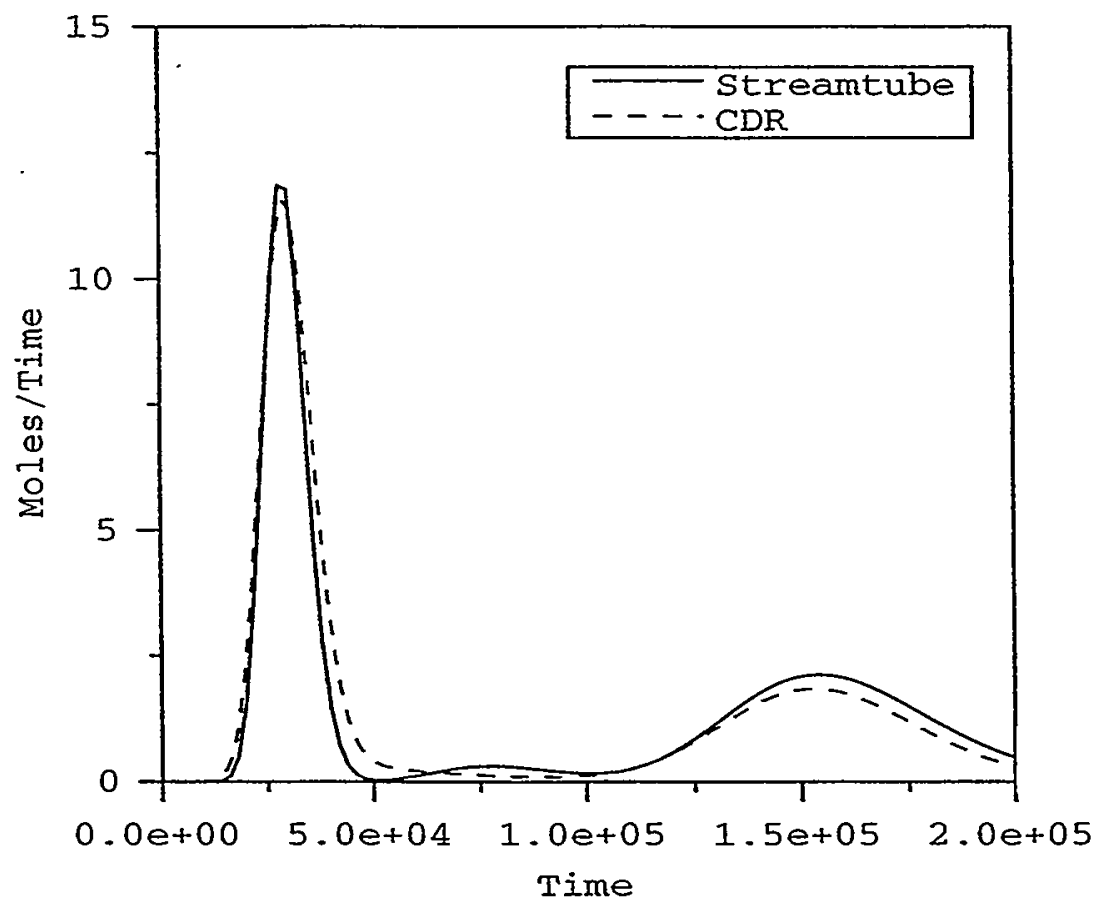

Figure 4-23. Flux averaged breakthrough curves comparing RST and CDR for two dimensional flow bypass problem with linear retadation in the high permeability layer. Fifty streamlines are used for this simulation.

streamlines for an accurate solution. The nearest neighbor approach could be replaced by a more complex interpolation scheme which might also alleviate the problem.

Table 4-13 shows that the 50 streamlines required by RST still results in considerably shorter simulation times than the CDR method. Adding a simple linear kinetic sorption reaction slows down the CDR method considerably. This is because FEHM must now access the reactive transport 
subroutines that are quite computationally intensive. We will see this effect is amplified for more complex reactive transport problems in section 4.5.

\begin{tabular}{|l|l|}
\hline \multicolumn{1}{|c|}{ Parameter } & \multicolumn{1}{c|}{ CPU Time (sec) } \\
\hline \hline CDR (10,201 node problem) & 4032 \\
\hline RST (20 streamlines) & 520 \\
\hline RST (50 streamlines) & 1300 \\
\hline
\end{tabular}

Table 4-13. Comparison of CPU times between CDR and RST for 2D vertical layer problem with linear sorption

\subsubsection{D Problem with Random Flow and Retardation}

In this example, we simulate the $2 \mathrm{D}$ random field problem but include linear retardation. Recall that permeability is distributed using a log normal distribution. To generate the random $\mathrm{Kd}$ field, we use a relationship developed by Smith et al. (1995)

$$
K_{d}=\frac{K_{d, a}}{\rho} \frac{\theta}{1-\theta} \cdot \sqrt{\frac{\theta}{2 \tau k}}
$$

where $K_{d, m}$ is the distribution coefficient based on the mass of rock, $K_{d, a}$ is the distribution coefficient based on surface area, $\rho$ is grain density, $\tau$ is tortuosity, $k$ is permeability, and $\theta$ is porosity. Equation 4-2 shows that $\mathrm{K}_{\mathrm{d}, \mathrm{m}}$ is inversely proportional to the square root of $\mathrm{k}$ for an ideal monominerallic porous media. Table 4-14 shows the parameters used in this simulation. Figure 4-24 shows the flux averaged breakthrough curve comparing the PTRK, CDR, and RST methods. The methods agree closely for this problem using 20 streamlines. Extracting $\mathrm{K}_{\mathrm{d}}$ using the nearest neighbor method is a good approximation for this case since $\mathrm{K}_{\mathrm{d}}$ is a random field without sharp discontinuities. Table 4-15 compares the CPU times of the three methods. The RST method again runs faster than the CDR method. 


\begin{tabular}{|l|l|}
\hline \multicolumn{1}{|c|}{ Parameter } & \multicolumn{1}{c|}{ Value } \\
\hline \hline Geometric Mean Permeability & $1 \times 10^{-16} \mathrm{~m}^{2}$ \\
\hline In(k) Variance & 1.0 \\
\hline $\mathrm{K}_{\mathrm{d}}$ geometric mean & $1.79 \mathrm{cc} / \mathrm{g}$ \\
\hline Correlation Length (isotropic) & $1 \mathrm{~m}$ \\
\hline Longitudinal dispersivity (for RST and CDR) & $0.1 \mathrm{~m}$ \\
\hline Transverse dispersivity (CDR only) & $0.01 \mathrm{~m}$ \\
\hline Molecular diffusion coefficient & $1 \times 10^{-10} \mathrm{~m}^{2} / \mathrm{s}$ \\
\hline Simulation time & $6 \times 10^{4} \mathrm{days}$ \\
\hline Time of pulse input at top boundary & $1000 \mathrm{day}$ \\
\hline Grid spacing & $0.1 \mathrm{~m}$ \\
\hline Surface area based distribution coeff., $\mathrm{K}_{\mathrm{d}, \mathrm{a}}$ & $1 \times 10^{-4} \mathrm{~m}^{2}$ \\
\hline tortuosity, $\tau$ & 0.5 \\
\hline grain density, $\rho$ & $1500 \mathrm{~kg} / \mathrm{m}^{3}$ \\
\hline
\end{tabular}

Table 4-14. Parameters for 2D random field problems

\begin{tabular}{|l|l|}
\hline \multicolumn{1}{|c|}{ Parameter } & \multicolumn{1}{c|}{ CPU Time (sec) } \\
\hline \hline CDR (10,201 node problem) & 5604 \\
\hline PTRK & 164 \\
\hline RST (20 streamlines) & 565 \\
\hline
\end{tabular}

Table 4-15. Comparison of CPU times between CDR, PTRK, and RST for 2D vertical layer problem with linear sorption

\subsection{D Multicomponent Transport Problem with Nonlinear Reactions}

\subsubsection{Migration of ${ }^{237} \mathrm{~Np}$}

For the next test problem, we simulate the transport of ${ }^{237} \mathrm{~Np}$ through the same random permeability field presented in sections 4.2.3 and 4.4.2. We use the migration of ${ }^{237} \mathrm{~Np}$ to test the effectiveness of the RST method for contaminants that participate in nonlinear reactions.The 


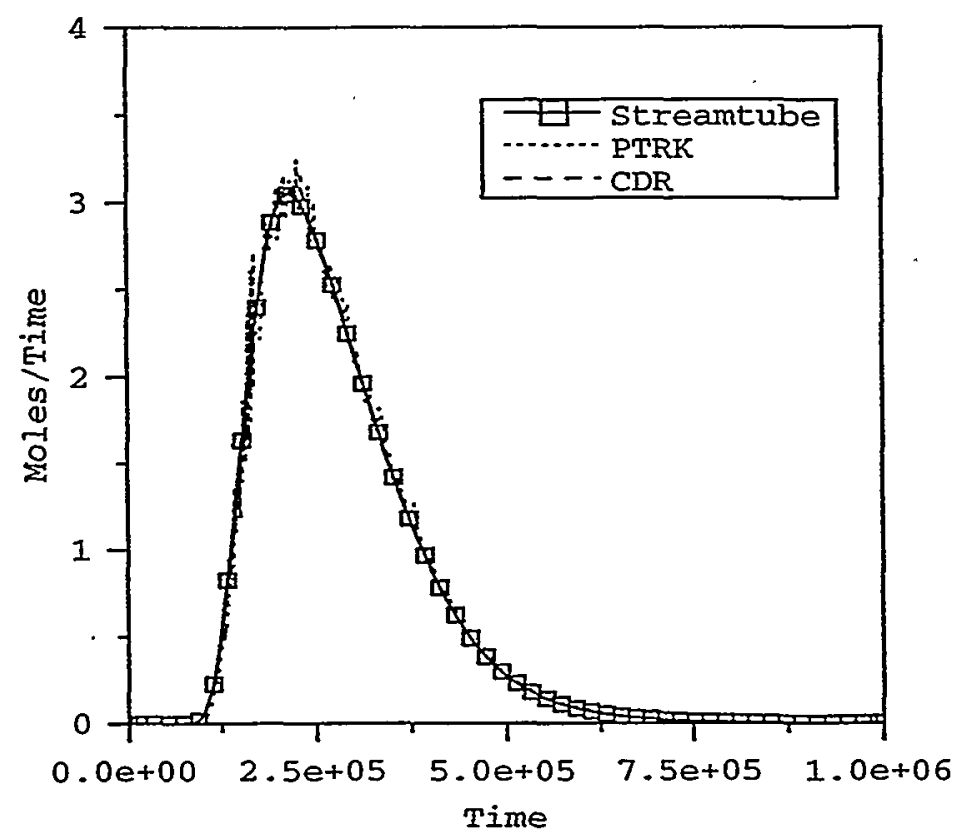

Figure 4-24. Two-dimensional random field problem with spatially correlated $\mathrm{K}_{\mathrm{d}}$. Twenty streamlines were used with RST.

domain size, boundary conditions and initial conditions are identical to sections 4.2.3 and 4.4.2. Characterization and performance assessment studies for the potential high-level nuclear waste repository at Yucca Mountain have identified ${ }^{237} \mathrm{~Np}$ as a radionuclide of concern for the proposed repository. Section 3.4 describes our model for ${ }^{237} \mathrm{~Np}$ migration in detail. Note that we use selective coupling as we described in section 3.4.

The geochemical processes we consider are the aqueous speciation of ${ }^{237} \mathrm{~Np}$ into nonsorbing carbonate/hydroxy complexes and the sorbing $\mathrm{NpO}_{2}^{+}$cation, and the sorption of $\mathrm{NpO}_{2}^{+}$ via an ion exchange mechanism. We assume that ${ }^{237} \mathrm{~Np}$ released into the aqueous phase participates in speciation reactions. These speciation reactions were given in Table (see also Viswanathan et al., 1998).

The ion-exchange reactions (3-4) and (3-5) are used to simulate the sorption of $\mathrm{NpO}_{2}^{+}$. The retardation of $\mathrm{NpO}_{2}^{+}$is correlated to the permeability at each location using equation 4-2 and the 
ion-exchange reactions 3-4 and 3-5. Specifically, equation $4-2$ relates permeability to the distribution coefficient, $\mathrm{K}_{\mathrm{d}}$. In this model, this $\mathrm{K}_{\mathrm{d}}$ represents the sorption of $\mathrm{NpO}_{2}^{+}$since this is the only form of ${ }^{237} \mathrm{~Np}$ that is assumed to sorb. For each value of permeability we can obtain a value for $\mathrm{K}_{\mathrm{d}}$ from equation 4-2. Using this $\mathrm{K}_{\mathrm{d}}$, the aqueous concentrations of $\mathrm{Na}$ and $\mathrm{Ca}$, and concentration of $\mathrm{Ca}-\mathrm{X}_{2}$, we can calculate a spatially variable concentration of $\mathrm{Na}-\mathrm{X}$ by solving a simple system of equations involving the mass action reactions for the ion exchange reactions. In effect, we correlate the permeability to the number of available sorption sites. Table 4-16, 4-17 and 4-18 show the parameters used in this simulation.

\begin{tabular}{|l|l|}
\hline \multicolumn{1}{|c|}{ Parameter } & \multicolumn{1}{c|}{ Value } \\
\hline \hline Geometric Mean Permeability & $1 \times 10^{-16} \mathrm{~m}^{2}$ \\
\hline In(k) Variance & 1.0 \\
\hline Correlation Length (isotropic) & $1 \mathrm{~m}$ \\
\hline Molecular diffusion coefficient & $1 \times 10^{-10} \mathrm{~m}^{2} / \mathrm{s}$ \\
\hline Longitudinal dispersivity (for RST and CDR) & 0.1 \\
\hline Transverse dispersivity (CDR) & 0.01 \\
\hline Simulation time & $1 \times 10^{6}$ days \\
\hline Time of pulse input of ${ }^{237} \mathrm{~Np}$ at top boundary & 1000 days \\
\hline
\end{tabular}

Table 4-16. Parameters for ${ }^{237} \mathrm{~Np}$ transport problem

\begin{tabular}{|l|l|l|}
\hline Component & Background Concentration & Injection Concentration \\
\hline \hline $\mathrm{pH}$ & 8.0 & 7.0 \\
\hline Carbonate & $2.52 \times 10^{-3} \mathrm{~mol} / \mathrm{L}$ & $2.52 \times 10^{-3} \mathrm{~mol} / \mathrm{L}$ \\
\hline${ }^{237} \mathrm{~Np}$ & $0.0 \mathrm{~mol} / \mathrm{L}$ & $1 \times 10^{-5} \mathrm{~mol} / \mathrm{L}$ \\
\hline $\mathrm{Na}$ & $5.43 \times 10^{-3} \mathrm{~mol} / \mathrm{L}$ & $5.43 \times 10^{-3} \mathrm{~mol} / \mathrm{L}$ \\
\hline $\mathrm{Ca}$ & $2.91 \times 10^{-4} \mathrm{~mol} / \mathrm{L}$ & $2.91 \times 10^{-4} \mathrm{~mol} / \mathrm{L}$ \\
\hline
\end{tabular}

Table 4-17. Aqueous component concentrations 
Note that we selectively couple using the strategy discussed in section 3.4 .

\begin{tabular}{|l|l|}
\hline Component & Background Concentration \\
\hline \hline $\mathrm{Na}-\mathrm{X}$ & spatially variable \\
\hline $\mathrm{Ca}-\mathrm{X}_{2}$ & $6.84 \times 10^{-5} \mathrm{~mol} / \mathrm{kg}$ rock \\
\hline
\end{tabular}

Table 4-18. Immobile component concentrations

Figure 4-25 compares the CDR and RST models. The methods agree closely although there are some discrepancies. Table 4-19 compares the CPU times of the CDR and RST method. For

\begin{tabular}{|l|l|}
\hline \multicolumn{1}{|c|}{ Parameter } & \multicolumn{1}{c|}{ CPU Time (sec) } \\
\hline \hline CDR (10,201 node problem) & 8602 \\
\hline RST (20 streamlines) & 2120 \\
\hline
\end{tabular}

Table 4-19. Comparison of CPU times between CDR and RST for 2D vertical layer problem with linear sorption

this reactive transport problem the RST method results in similar computational savings compared to the nonreactive verification problems in previous sections. Specifically, RST is about 4 times faster than CDR. 


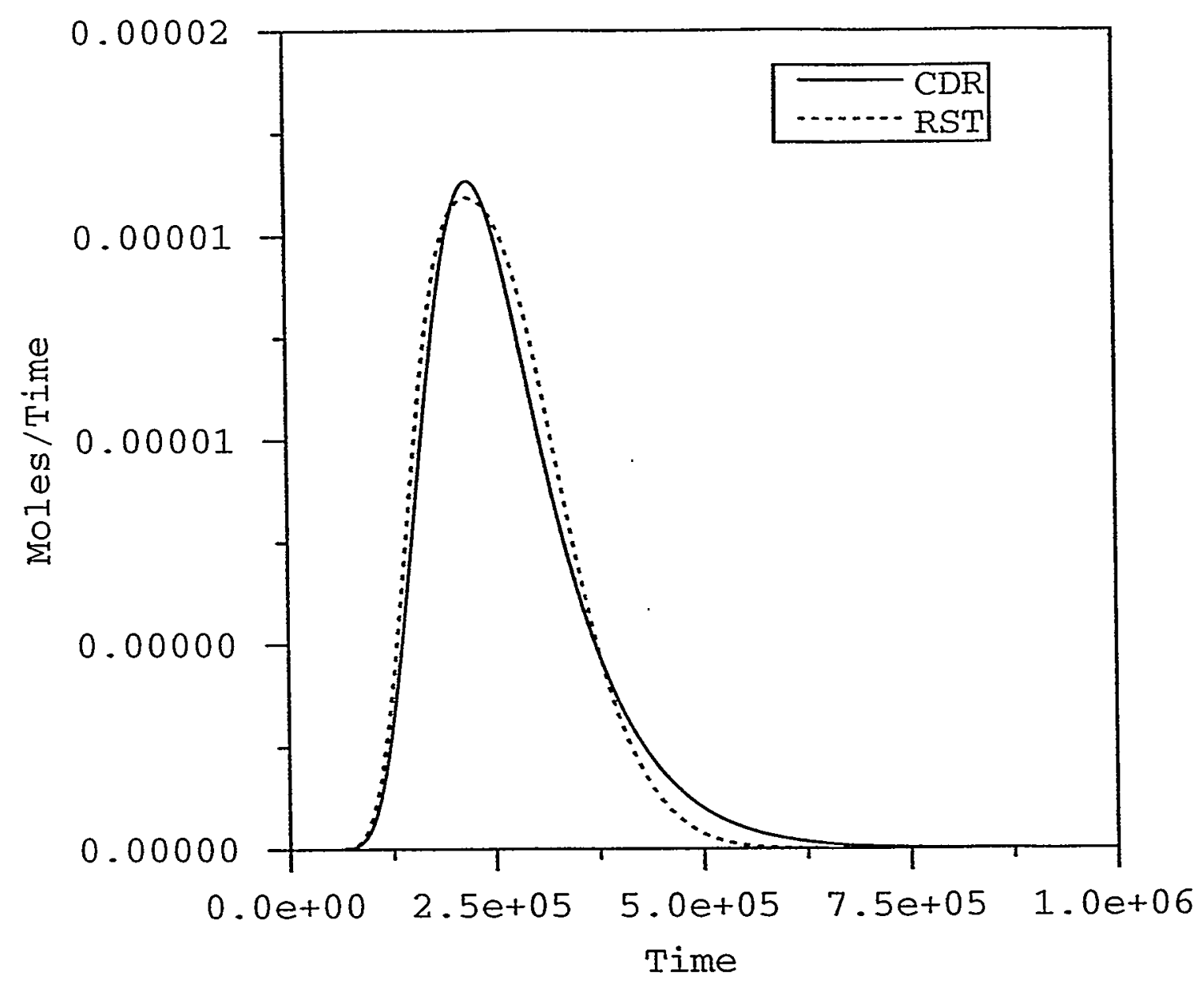

Figure 4-25. Comparison of the RST and CDR models for two-dimensional ${ }^{237} \mathrm{~Np}$ transport with nonlinear reactions. 


\section{CALICO HILLS SIMULATIONS}

\subsection{Summary}

In this chapter, we utilize the CDR and RST techniques to simulate ${ }^{237} \mathrm{~Np}$ transport through the Calico Hills unit, a key barrier to radionuclide transport in the unsaturated zone of Yucca Mountain. Characterization and performance assessment studies for the potential high-level nuclear waste repository at Yucca Mountain have identified ${ }^{237} \mathrm{~Np}$ as a radionuclide of concern for the proposed repository (Viswanathan et al., 1998). This chapter serves to test the methods for a practical application that is of interest to the Yucca Mountain Project.

The distribution of minerals such as zeolites can strongly influence the flow patterns of percolating fluids through the Calico Hills unit. The sorption of many radionuclides (e.g. ${ }^{237} \mathrm{~Np}$ ) are also strongly controlled by the mineral distribution in the flow pathways between the repository and the water table. The site-scale model of Yucca Mountain (Robinson et al., 1997) relies on averaging techniques in order to model the large scales which are of interest in the flow and transport calculations. In this chapter we test whether small scale variability in the zeolitic mineral distribution in the Calico Hills can have a large impact on the migration of ${ }^{237} \mathrm{~Np}$. Using the CDR and RST techniques, we examine a two-dimensional $64 \mathrm{~m}$ by $64 \mathrm{~m}$ section of the Calico Hills to study radionuclide transport in greater detail. The selective coupling method (developed in Chapter 2) is used to model the reactive transport of ${ }^{237} \mathrm{~Np}$ for both the CDR method and for each onedimensional reactive transport simulation used as part of the RST method. We show that the RST method is an accurate and efficient method that can be used to assess the effects of chemical and physical heterogeneity on radionuclide transport in the unsaturated zone. 


\subsection{Motivation}

Natural spatial variability in hydrologic and chemical properties can play an important role in the transport of contaminants in groundwater. Stochastic techniques to study the effects of this small-scale variability make up a significant part of the groundwater literature (Gelhar, 1993). For example, reduction in sorption due to physical and chemical heterogeneity has been described by Tompson et al. (1996) and Smith et al. (1996). They suggest that for specific saturated flow systems, significant contaminant transport will occur in zones of minimal reactivity since permeability and reactivity were found to be inversely correlated. Since we are concerned with zeolite heterogeneity in the unsaturated zone, the relationship between reactivity and effective permeability must be considered rather than the relationship between saturated permeability and reactivity.

The site-scale model of Yucca Mountain (Robinson et al., 1997) relies on averaging techniques in order to model the large scales which are of interest in the flow and transport calculations. In this chapter, we study the impact of small-scale variability in the zeolite

distribution on the transport of ${ }^{237} \mathrm{~Np}$ through the unsaturated zone. Specifically, ${ }^{237} \mathrm{~Np}$ forms numerous aqueous complexes of which some participate in ion-exchange reactions with the zeolites, resulting in retardation of ${ }^{237} \mathrm{~Np}$ (see section 3.4).

Another assumption made in previous studies (e.g. Robinson et al, 1997) was to use a threshold cutoff for zeolitic abundance. The threshold provides a method for assigning either vitric or zeolitic properties to each node since correlations did not exist between the degree of zeolitization and the hydrologic and chemical properties. We replace the zeolitic threshold method with more rigorous geostatistical modeling. The underlying statistical information for zeolitic distributions is obtained from the Yucca Mountain Mineralogic Model described in Chapter 12 of Robinson et al. (1997). This will allow us to determine whether using a threshold cutoff for zeolitic abundance is an appropriate upscaling method for characterizing small scale heterogeneity. Figure 5-1 a shows the zeolitic distribution when a $10 \%$ threshold is used as the basis for considering a 
node to be zeolitically altered. The $10 \%$ threshold results in a zeolitic distribution that roughly follows the layers. A potential problem with using a simple threshold is that the zeolitic distributions change significantly as a function of the threshold. Figure 5-1b shows the zeolitic distribution for a $20 \%$ threshold: the layers loose their continuity and in some places appear as lenses. We examine the assumption of using a cutoff for zeolitic abundance by comparing those results to geostatistical simulations in which saturated permeability, characteristic curve parameters for relative permeability, and reactivity are correlated to zeolitic abundance. With such correlations, the effects of chemical and physical heterogeneity can be treated more rigorously without having to rely on a threshold cutoff for zeolitic abundance.

This chapter will summarize the ongoing efforts to examine the effects of chemical and physical heterogeneity on ${ }^{237} \mathrm{~Np}$ transport.

The following topics are covered in this chapter:

- A simple example to illustrate the implications of the choice of the threshold value for zeolitic cutoff.(section 5.3);

- We present field data that support a correlation between the physical and chemical properties at Yucca Mountain indicating that these correlations can have a large impact on the travel times for ${ }^{237} \mathrm{~Np}$. These correlations are used to study the relationship between zeolitic abundance, van Genuchten parameters, permeability and reactivity (section 5.4);

- Geostatistical techniques are used in conjunction with the mineralogical model to develop variograms, kriging maps, and conditional simulations of zeolitic abundance;

- RST and CDR are used to examine a $64 \mathrm{~m}$ by $64 \mathrm{~m}$ section of the Calico Hills unit.

\subsection{Implications of Zeolite Threshold Cutoff}

A simple example using the CDR model is developed in this section to illustrate the effect of using a threshold cutoff for zeolitic abundance on the transport of ${ }^{237} \mathrm{~Np}$. Transport through a 
a

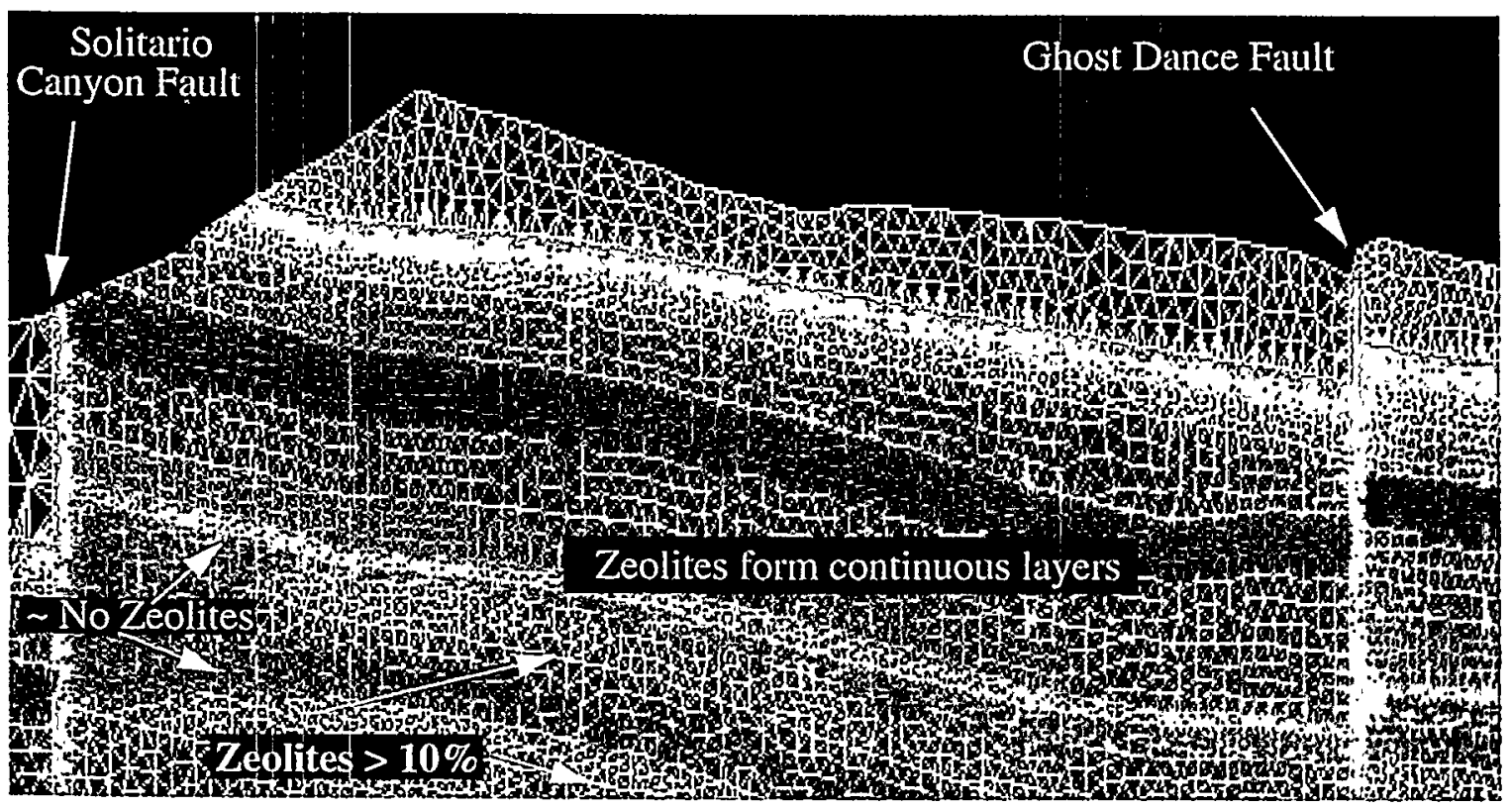

b

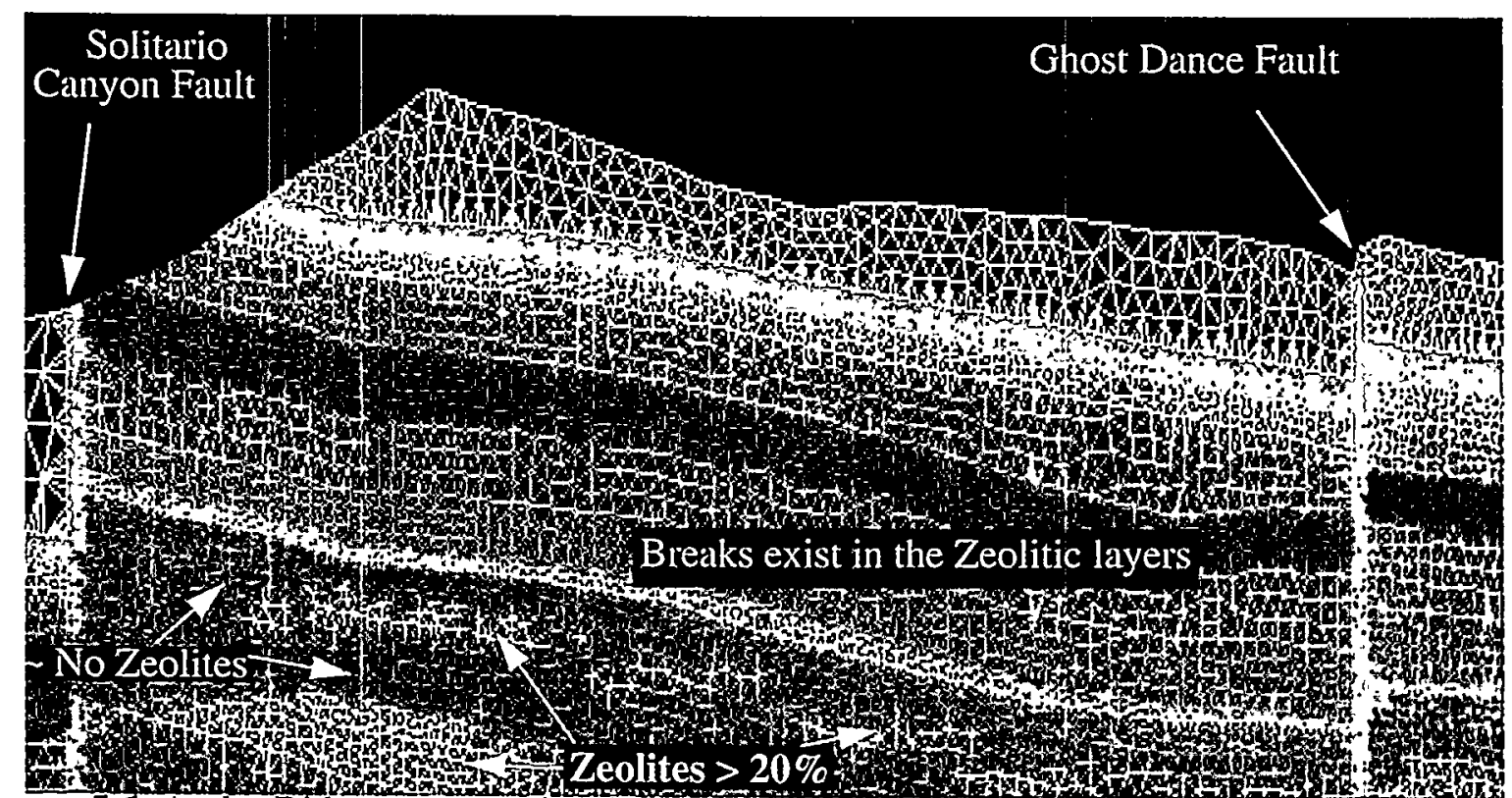

Figure 5-1. Antler Ridge cross section (a) hydrologic zones including rock with greater than $10 \%$ zeolitic abundance, (b) hydrologic zones including rock with greater than $20 \%$ zeolitic abundance.

$10 \mathrm{~m}$ by $10 \mathrm{~m}$ block of unfractured vitric tuff with a zeolitic layer will be compared to a block of vitric tuff containing a rectangular zeolitic lens (Figure 5-2). Each block is representative of a typical element in the site-scale model for the Calico Hills unit. The layered case is analogous to a zeolitic layer shown in Figure 5-1a, whereas, the lens case is used to illustrate the case of a 
discontinuous zeolite distribution as shown in Figure 5-1b. In these block models, care must be taken to choose boundary conditions which will simulate a single element of the site-scale model. In the site-scale model, the water table serves as the bottom boundary condition and is thus simply set to a saturation of one. At steady state, the capillary pressure gradient and gravitational forces equilibrate to establish a saturation profile throughout the model domain. Due to the large size of the site-scale model, the capillary pressure gradient across a typical element is small. The balance between capillary pressure, which acts to retain water in the pores, and gravitational forces leads to a net downward percolation.

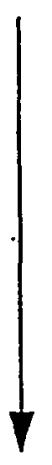

Gravity Driven Flow

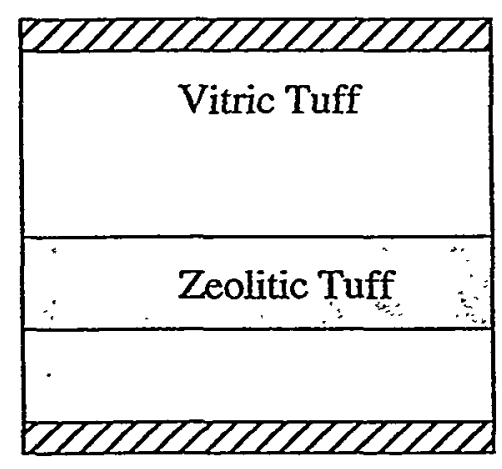

Layered Case

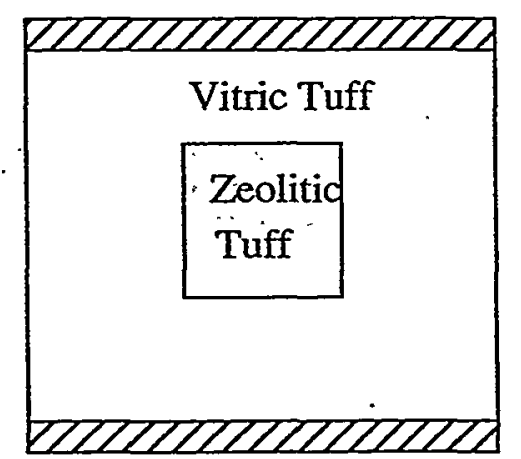

Lens Case

\section{$\square$ constant capillary pressure boundary}

Figure 5-2. Two possible zeolitic geometries at the subgrid scale $(10 \mathrm{~m}$ by $10 \mathrm{~m})$.

In the block models, the bottom boundary cannot be set to a saturation of one as in sitescale models because the block represents an element from the Calico Hills unit which is hundreds of meters above the water table. Therefore, we set the top and bottom boundaries of the block model to a constant capillary pressure, which results in a steady infiltration rate through the block. Lateral flow across an element can be important in the site-scale model but is not considered in this example. The left and right sides are no-flow boundaries. We assume that these blocks are in a region of the site-scale model where downward percolation dominates. 
Table shows the properties of the vitric and zeolitic tuffs, and Figure 5-3 shows the relative permeability characteristic curves obtained from the van Genuchten model (van Genuchten, 1980). Model parameters (Flint, 1996) are given in Table 5-1.

\begin{tabular}{|l|l|l|}
\hline Parameter & Vitric Tuff & Zeolitic Tuff \\
\hline saturated hydraulic conductivity $@ 25^{\circ} \mathrm{C},(\mathrm{m} / \mathrm{s})$ & $4.3 \times 10^{-9}$ & $7.03 \times 10^{-11}$ \\
\hline saturated permeability, $\mathrm{k}_{\text {sat }}\left(\mathrm{m}^{2}\right)$ & $4.4 \times 10^{-16}$ & $7.17 \times 10^{-18}$ \\
\hline$\alpha$ of van Genuchten model $(1 / \mathrm{m})$ & 0.0267 & 0.0035 \\
\hline $\mathrm{n}$ of van Genuchten model & 1.386 & 1.495 \\
\hline porosity, $\phi$ & 0.27 & 0.29 \\
\hline
\end{tabular}

Table 5-1. Hydrologic and chemical propertiesof vitric and zeolitic tuff

The quantity $k_{\text {sat }} * R_{l}$ is the effective permeability of the unsaturated medium where $k_{\text {sat }}$ is the saturated permeability and $R_{1}$ is the relative permeability. The characteristic curves of $k_{\text {sat }} * R_{I}$ versus capillary pressure show that, as expected, zeolitic tuff is less permeable than vitric tuff for capillary pressures less than $100 \mathrm{~m}$. However, for capillary pressures greater than $100 \mathrm{~m}$, the zeolitic tuff is actually more permeable than the vitric tuff. The permeability trends can be explained by the saturation-capillary pressure plot (Figure 5-3). At low capillary pressures, the
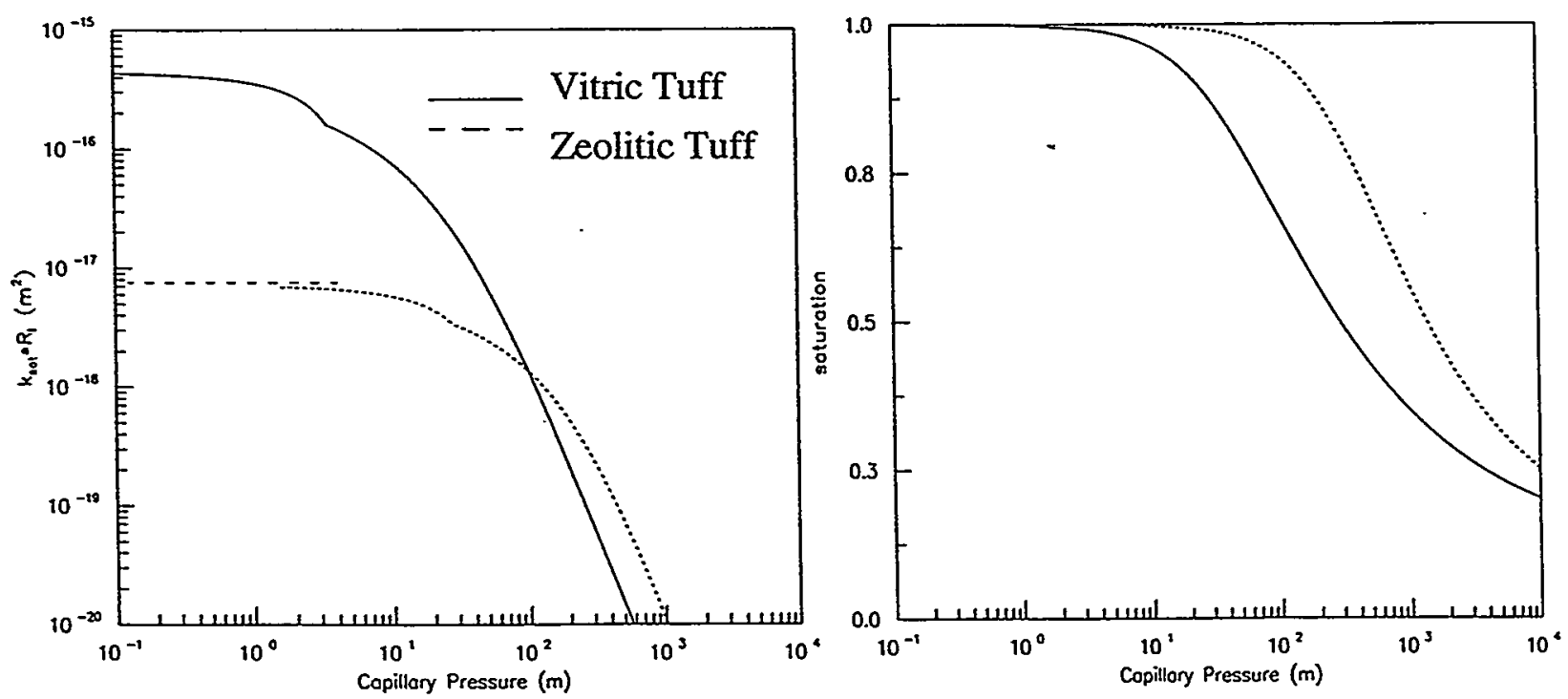

Figure 5-3. Comparison of the characteristic curves for zeolitic and vitric tuff. 
zeolitic and vitric tuffs are highly saturated and the permeabilities of the media approach the saturated permeability values. However, at a high capillary pressure, both media are drier, allowing the zeolitic tuff to actually conduct as much flow (or slightly) as the vitric tuff.

At steady state, the capillary pressure and gravitational forces equilibrate, leading to a net downward infiltration through the media. The capillary pressure at the model boundaries can therefore be chosen to obtain a given infiltration rate throughout the media. For example, setting the capillary pressure difference to $24 \mathrm{~m}$ results in an infiltration rate of about $4 \mathrm{~mm} / \mathrm{yr}$, whereas, a capillary pressure of $155 \mathrm{~m}$ results in an infiltration rate of about $0.1 \mathrm{~mm} / \mathrm{yr}$. Note that the infiltration rates are slightly different for the lens and layer cases for a given capillary pressure but are close enough for this illustrative example.

Three simulations were conducted at infiltration rates of $4 \mathrm{~mm} / \mathrm{yr}, 1 \mathrm{~mm} / \mathrm{yr}$ and $0.1 \mathrm{~mm} / \mathrm{yr}$ (Figure 5-4). For the $0.1 \mathrm{~mm} / \mathrm{yr}\left(\mathrm{P}_{\text {cap }}=155 \mathrm{~m}\right)$ simulation, the velocities are actually higher through the zeolites than through the vitric tuff, resulting in preferential flow through the zeolites. At $1 \mathrm{~mm} / \mathrm{yr}\left(\mathrm{P}_{\text {cap }}=88 \mathrm{~m}\right)$, a slight bypass occurs around the zeolites with preferential flow through the vitric tuff. Again, the velocities through both media are similar. However, at $4 \mathrm{~mm} / \mathrm{yr}\left(\mathrm{P}_{\text {cap }}=\right.$ $24 \mathrm{~m}$ ), velocities are much higher through the vitric tuff than the zeolitic tuff. These trends are a direct result of the $k_{\text {sat }} * R_{1}$ vs. capillary pressure curve (Figure 5-3). Interestingly, the unsaturated permeabilities of the media change drastically over the range of infiltration rates that are expected at Yucca Mountain.

After a steady state flow field is obtained, ${ }^{237} \mathrm{~Np}$ is injected uniformly along the top boundary to simulate the downward migration of ${ }^{237} \mathrm{~Np}$ from the repository. For simplicity, ${ }^{237} \mathrm{~Np}$ is assumed to linearly sorb to the zeolitic tuff with an equivalent $\mathrm{K}_{\mathrm{d}}$ of $2 \mathrm{~cm}^{3} / \mathrm{g}$, and to not sorb to the vitric tuff. Note that a $K_{d}$ of $2 \mathrm{~cm}^{3} / g$ is the mean $K_{d}$ measured by Triay et al. (1996b) for ${ }^{237} \mathrm{~Np}$ onto zeolitic tuffs. In both blocks zeolites comprise $25 \%$ of the domain with the difference being the geometry of the zeolite (Figure 5-2). Table shows the parameters used to conduct the 


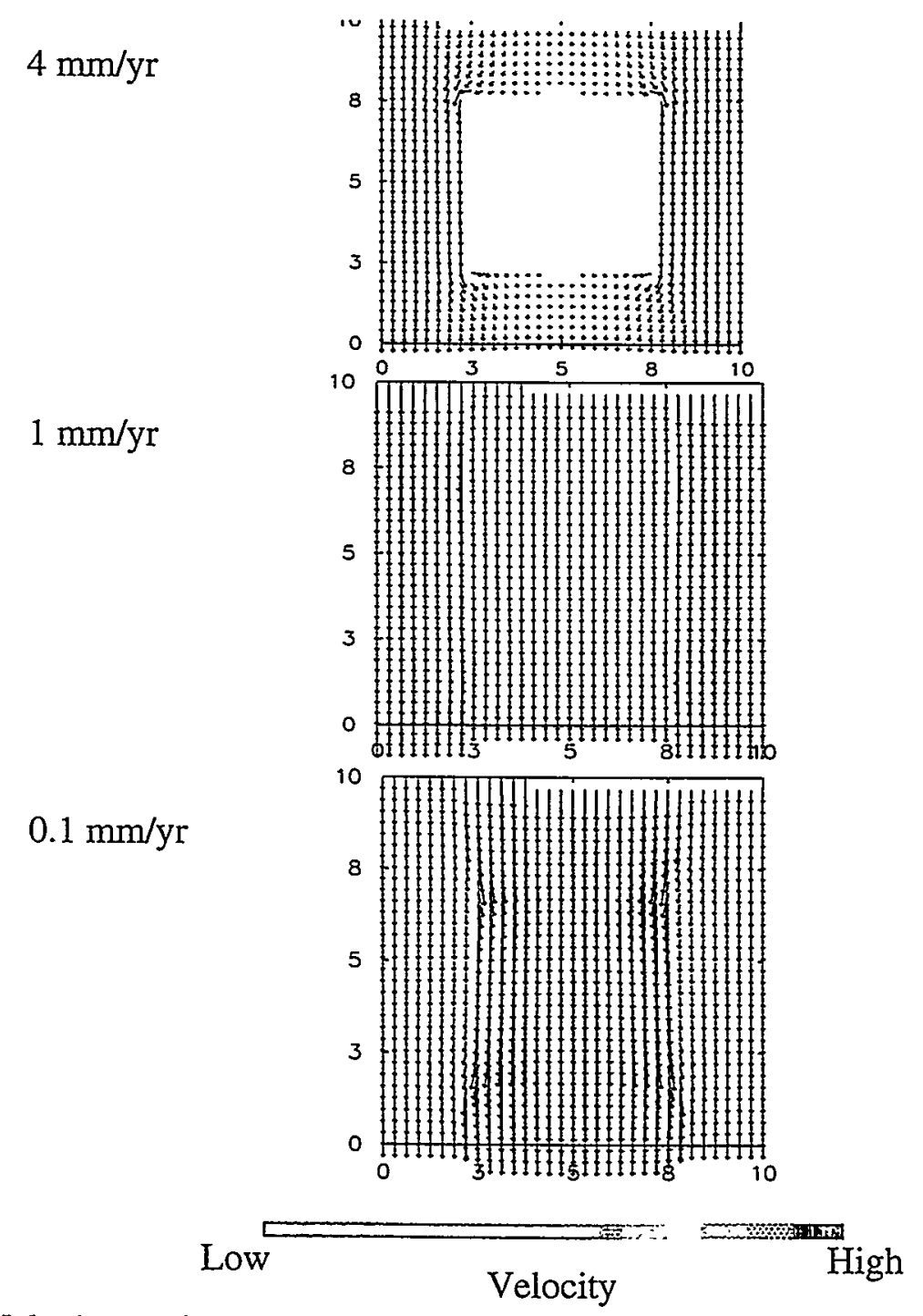

Figure 5-4. Velocity profile for the zeolitic lens case at three different infiltration rates.

simulations. The dispersivity and diffusion parameters are hypothetical values but are appropriate for ${ }^{237} \mathrm{~Np}$ in Calico Hills tuffs.

\begin{tabular}{|l|l|}
\hline Molecular Diffusion, $\mathrm{D}^{*}$ & $1 \times 10^{-10} \mathrm{~m}^{2} / \mathrm{s}$ \\
\hline Longitudinal Dispersivity, $\mathrm{a}_{\mathrm{l}}$ & $0.1 \mathrm{~m}$ \\
\hline Transverse Dispersivity, $\mathrm{a}_{\mathrm{t}}$ & $0.01 \mathrm{~m}$ \\
\hline Grid spacing, $\Delta \mathrm{x}$ & $0.3125 \mathrm{~m}$ \\
\hline Time step, $\Delta \mathrm{t}$ & 5,000 days \\
\hline
\end{tabular}

Table 5-2. Parameters used for layer-bypass simulations 
Figure 5-5 compares the cumulative mass of ${ }^{237} \mathrm{~Np}$ arriving at the bottom boundary for the layered and the lens cases. At each infiltration rate, a pulse of ${ }^{237} \mathrm{~Np}$ was injected into the system at the beginning of each simulation. Specifically, 166 moles of ${ }^{237} \mathrm{~Np}$ at $4 \mathrm{~mm} / \mathrm{yr}, 60 \mathrm{moles}$ at 1 $\mathrm{mm} / \mathrm{yr}$ and $11 \mathrm{moles}$ at $0.1 \mathrm{~mm} / \mathrm{yr}$ were injected over a 10 year period. For cases of lower infiltration rates, the layered case and lens case behave similarly since there are no major preferential pathways. However, for the $4 \mathrm{~mm} / \mathrm{yr}$ case, the ${ }^{237} \mathrm{~Np}$ is retarded to a greater extent for the layered case than for the lens case. This set of simple simulations shows that at relatively high infiltration rates, more extensive bypassing will occur if there are breaks in the zeolitic layers. Specifically, flow will preferentially avoid the zeolitic areas at relatively high infiltration rates. In this example, rock was assigned either zeolitic or vitric properties. This assumption is analogous to using the $10 \%$ zeolitic cutoff value.

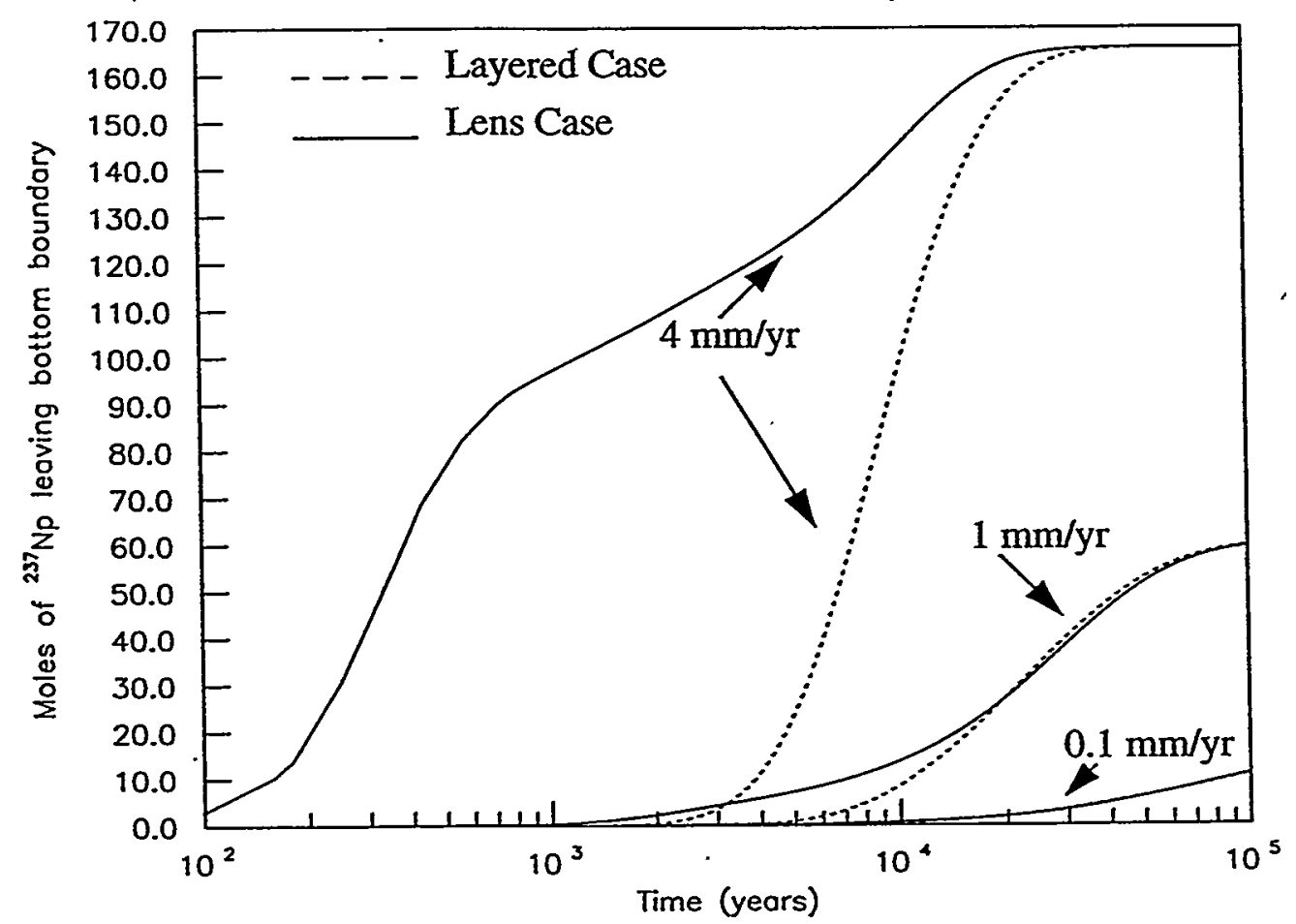

Figure 5-5. Comparison of ${ }^{237} \mathrm{~Np}$ breakthrough curves for the layer and lens cases. 


\subsection{Relationships Between Physical and Chemical Heterogeneity at Yucca Mountain}

Figure 5-1 showed how different threshold cutoffs for zeolitic abundance can result in significantly different zeolite distributions. In previous studies (e.g. Robinson et al., 1997), a particular threshold was chosen for simplicity, and also because correlations did not exist between the degree of zeolitization and the hydrologic and chemical properties. Therefore, the cutoff method was used to tag each spatial location as either vitric tuff or zeolitic tuff.

In reality, the percent of zeolitic alteration is a continuous property. In this section, we distribute zeolitic abundance using statistical information from the mineralogic model (Chipera et al., 1997a,b). We then examine various sources of field data to correlate hydrologic (e.g. permeability and characteristic curve parameters) and chemical properties (e.g., distribution coefficient $\mathrm{K}_{\mathrm{d}}$ ) of the tuffs to zeolitic abundance. Evidence of zeolitic alteration controlling these properties is presented in Robinson et al. (1997). Such correlations are expected because the physical and chemical properties of a geologic medium can often be related back to fundamental measurable properties such as grain or pore size (Smith et al, 1996). Specifically, the process of zeolitic alteration acts to reduce the pore sizes of the vitric tuffs. If such correlations could be developed it would eliminate the need for using a threshold cutoff.

The correlations we use are a combination of empirical relationships obtained from field data and theoretical relationships. Several regression formulas have been developed by various researchers within the Yucca Mountain Project. Physically-based theoretical relationships also exist and are used to correlate the various properties. The following subsections describe relationships that are used to generate realizations of multiply-correlated properties.

\subsubsection{Distributing Zeolitic Abundance}

Percent zeolitic alteration is distributed throughout a $64 \mathrm{~m} \mathrm{x} 64 \mathrm{~m}$ block using a random field generator (Tompson et al., 1989). We use a normal distribution to distribute the percentage of zeolitic abundance simply clipping values less than 0 and greater than 100 . The mean and 
variance of zeolitic abundance are obtained from the mineralogical model (Chipera et al., 1997a,b). The mean of zeolitic abundance is $27 \%$ with a standard deviation of $30 \%$. An isotropic correlation length of $1 \mathrm{~m}$ using the exponential model. For these small scale simulations we do not perform conditional simulations. Robinson et al. (1997) documents conditional geostatistical simulations performed using the mineralogic model. The CDR model was then used with the Los Alamos Integrated Site Scale Model to perform ${ }^{237} \mathrm{~Np}$ migration studies at the site scale $(2 \mathrm{~km}$ wide by about $400 \mathrm{~m}$ high). In this thesis, we will focus on smaller scale variabilities and simulations which utilize both the CDR and RST methods.

\subsubsection{Correlation between Permeability and Zeolitic Abundance}

Flint (1996) developed a relationship between $\mathrm{K}_{\text {sat }}$ and the percent of clinoptilolite, the main zeolitic mineral in the Calico Hills unit (Figure 5-6). The data set is limited because data points exist at low and high percentages of zeolitic abundance, but not at intermediate percentages. The regression relationship clearly shows that there is not a $1: 1$ relationship between $\mathrm{K}_{\text {sat }}$ and the percent zeolite. However, zeolitic alteration does clearly lower the permeability of the tuff. Others have also shown that zeolitic alteration causes a large reduction in the hydraulic conductivity (Loeven, 1993). Although the correlation coefficient of the regression is low, it captures a trend that is physically meaningful.

\subsubsection{Correlation Between Permeability and Relative Permeability}

The van Genuchten function is used for predicting capillary pressure (van Genuchten, 1980). Qualitatively, $\alpha$ is inversely related to the air entry pressure, and $n$ is inversely related to the width of the pore-size distribution. Therefore, a theoretical correlation between permeability and relative permeability may be possible. Although no theoretical correlation has been found, an empirical relationship exists between $\alpha$ and permeability. Altman et al. (1996) performed a linear regression between the hydraulic conductivity $\left(\mathrm{K}_{\text {sat }}\right)$ and $\alpha$ using core data from the Calico Hills unit.

$$
\log (\alpha)=-1.62+0.41 \log \left(K_{s a l}\right) \quad r^{2}=0.81
$$




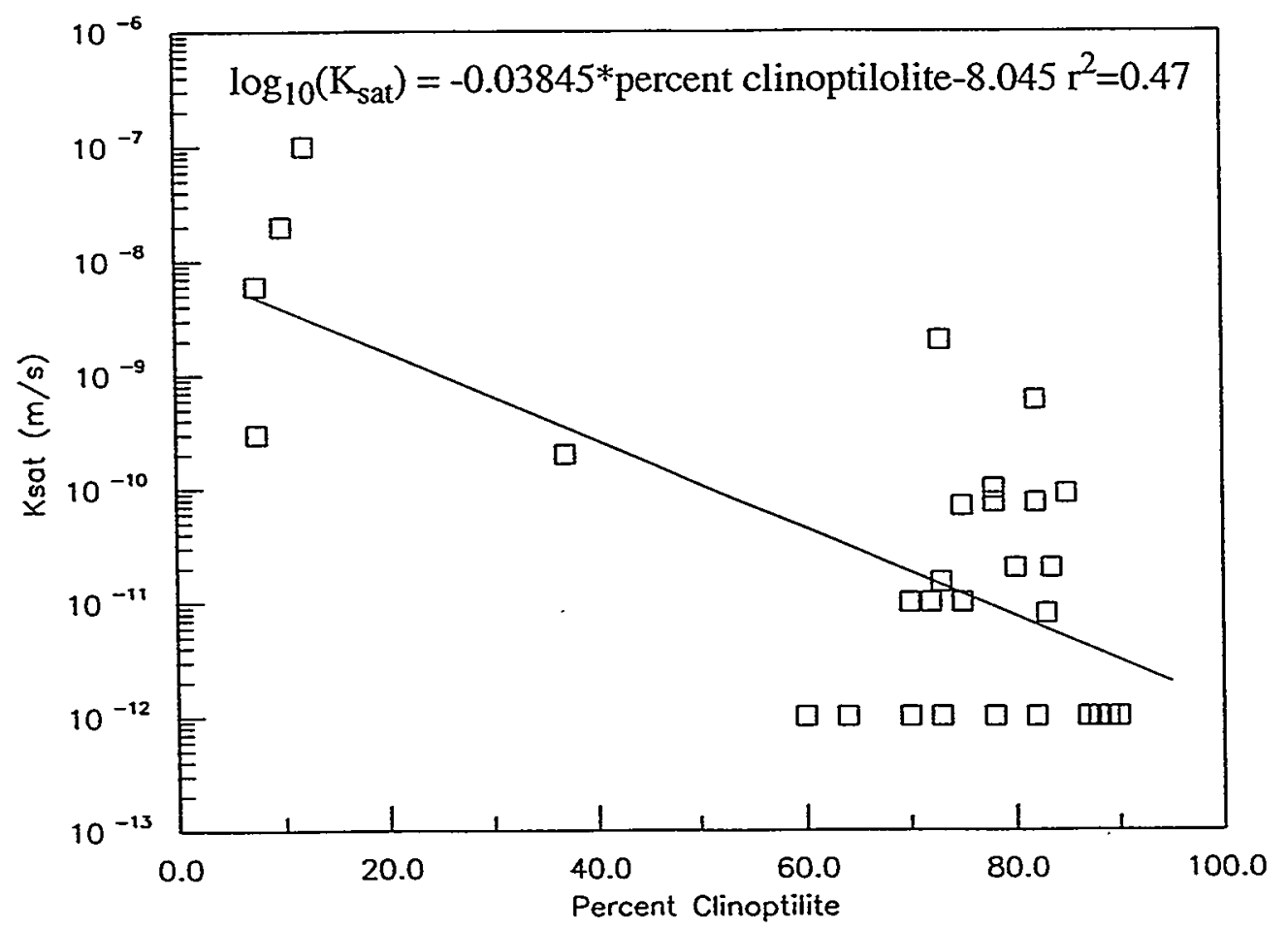

Figure 5-6. Data relating percent clinoptilolite to $K_{\text {sat, }}$ (from Flint, 1996).

where $\alpha$ is in units of $1 / \mathrm{Pa}$ and $\mathrm{K}_{\mathrm{sat}}$ is in units of $\mathrm{m} / \mathrm{s}$ (Figure 5-7). Since the range of $\mathrm{n}$ is only from 1.21 to 1.73 , Altman et al (1996) suggest that a constant mean value of 1.60 can be used. A constant residual saturation of 0.0453 is assumed for the Calico Hills tuffs. This parameter is obtained via fits to data rather than direct measurement, and thus is very poorly constrained. For this reason, we hold the value constant in the simulation.

\subsubsection{Correlation Between Permeability and Reactivity}

The retardation of $\mathrm{NpO}_{2}^{+}$is correlated to the permeability at each location using equation 4-2 and the ion-exchange reactions 3-4 and 3-5. This procedure is described in detail in section 4.5.1. 


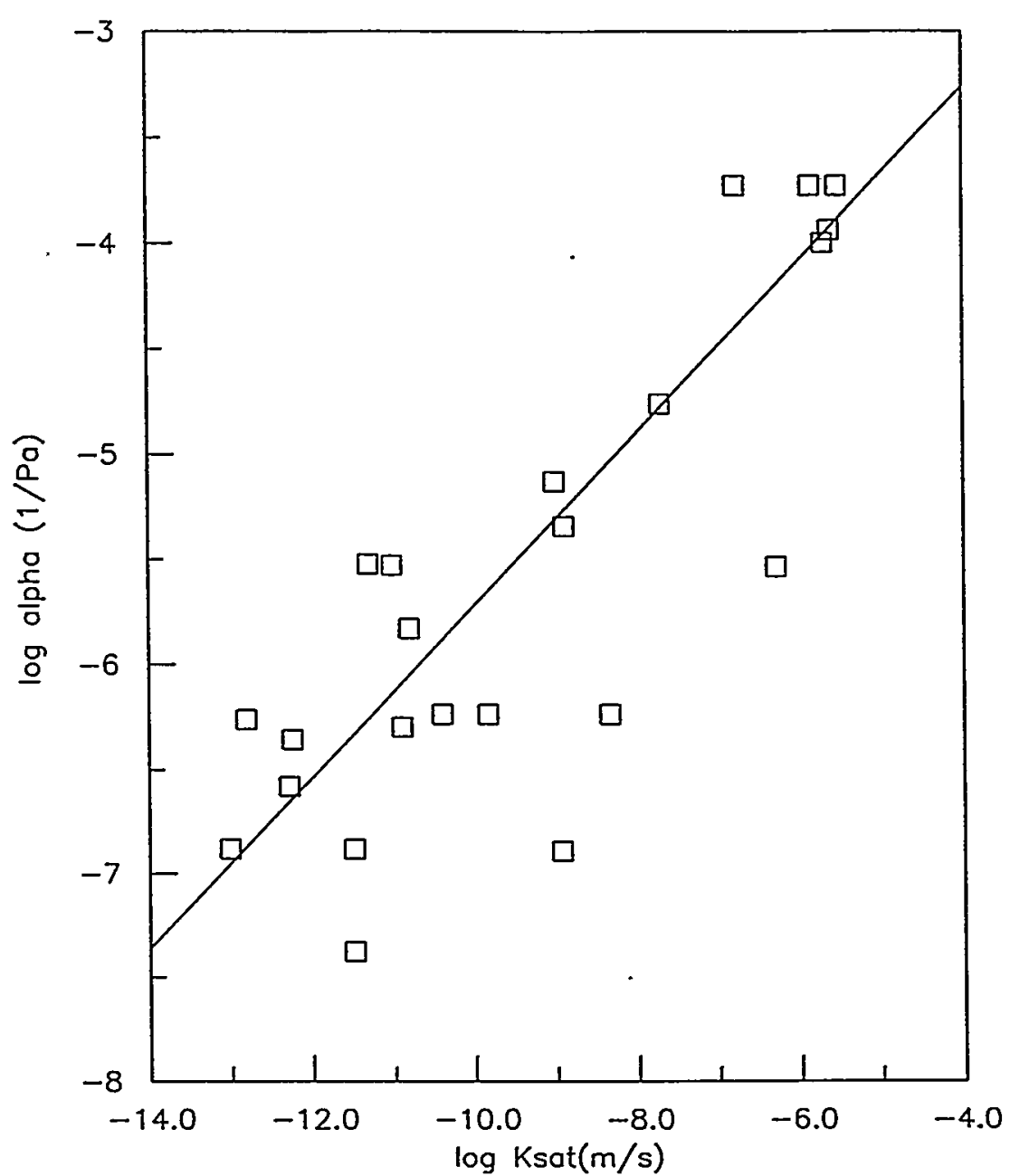

Figure 5-7.Data from Altman et al. (1996) relating $\alpha$ to $\mathrm{K}_{\mathrm{sat}}$.

\subsection{Simulations Utilizing CDR and RST}

\subsubsection{Summary}

In this section, we study radionuclide transport through a $64 \mathrm{~m}$ by $64 \mathrm{~m}$ portion of the Calico Hills using the RST and CDR methods. We use the above correlations to relate percent zeolitic alteration to other physical properties such as permeability, the $\alpha$ parameter in the van Geneuchten model, and reactivity. FEHM is used to obtain a steady state flow solution through the block, assuming that flow is gravity driven from the top of the block to the bottom. This flow solution is then used with the CDR and RST models to simulate ${ }^{237} \mathrm{~Np}$ transport. 
As with the simulations conducted in section 5.3, care must be taken to choose boundary conditions. In the site-scale model, the water table serves as the bottom boundary condition and is thus simply set to a saturation of one. At steady state, the capillary pressure gradient and gravitational forces equilibrate to establish a saturation profile throughout the model domain. Due to the large size of the site-scale model, the capillary pressure gradient across a typical element is small. The balance between capillary pressure, which acts to retain water in the pores, and gravitational forces leads to a net downward percolation. Figure 5-8 shows the realization of zeolitic abundance random field used for these simulations. In this scaled down portion of the

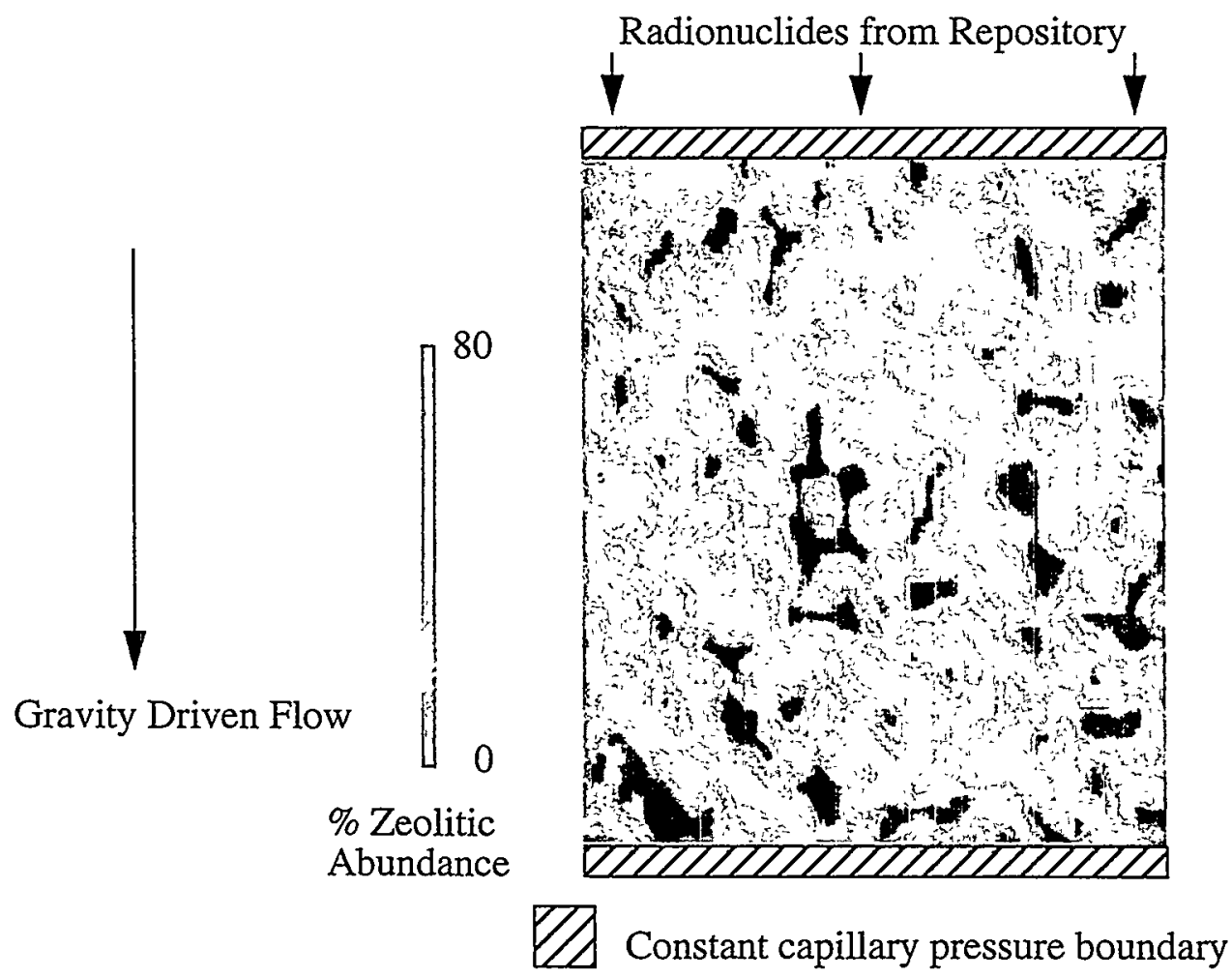

Figure 5-8. Schematic of $64 \mathrm{~m}$ by $64 \mathrm{~m}$ Calico Hills simulations.

Calico Hills, the bottom boundary cannot be set to a saturation of one as in site-scale models, because the $64 \mathrm{~m}$ by $64 \mathrm{~m}$ block of the Calico Hills unit is above the water table. Therefore, we set the top and bottom boundaries of the block model to a constant capillary pressure, which results in a steady infiltration rate through the block. Lateral flow across an element can be important in the site-scale model but is not considered in this example. The left and right sides are no-flow 
boundaries. We assume that the block is in a region of the site-scale model where downward percolation dominates. Table 5-3 shows the parameters used for these simulations.

\begin{tabular}{|l|l|}
\hline Problem Domain & $64 \mathrm{~m}$ by $64 \mathrm{~m}$ \\
\hline CDR Grid spacing, $\Delta \mathrm{x}$ & $0.5 \mathrm{~m}$ \\
\hline Maximum CDR Time step, $\Delta \mathrm{t}$ & 5,000 days \\
\hline Molecular Diffusion, $\mathrm{D}^{*}$ & $1 \times 10^{-10} \mathrm{~m}^{2} / \mathrm{s}$ \\
\hline Longitudinal Dispersivity, $\mathrm{a}_{\mathrm{l}}$ & $1 \mathrm{~m}$ \\
\hline Transverse Dispersivity, $\mathrm{a}_{\mathrm{t}}$ & $0.1 \mathrm{~m}$ \\
\hline Mean of Zeolitic Abundance & $27 \%$ \\
\hline Standard Deviation Zeolitic Abundance & $30 \%$ \\
\hline Correlation length & $1 \mathrm{~m}$ \\
\hline
\end{tabular}

Table 5-3. Parameters used in $64 \mathrm{~m}$ by $64 \mathrm{~m}$ Calico Hills simulations

\subsubsection{Nonreactive Transport Simulations (Technecium)}

First we compare CDR and RST using a nonreactive solute for a low $(0.1 \mathrm{~mm} / \mathrm{yr})$ and high

- (4 mm/yr) infiltration scenario.Radionuclides such as ${ }^{99} \mathrm{Tc}$ are expected to transport without retardation through the Calico Hills unit (Triay, 1996a).

Figure 5-9 shows the saturation and velocity profiles for the $0.1 \mathrm{~mm} / \mathrm{yr}$ infiltration scenario. At the low infiltration case, the zeolitic and nonzeolitic regions exhibit similar relative permeabilities. Recall that we witnessed this behavior in section 5.3. Therefore, the velocity profile through the block is very uniform. Figure 5-10a shows the flow field decomposed into sixteen streamlines which make up eight equal flow streamtubes. Since, the velocity profile is so uniform, the streamtubes do not contract or expand much throughout the problem domain. Figure 5-10b compares the flux averaged tracer breakthrough at the bottom of the model for the CDR and RST method. The plot also shows the individual breakthrough curves for each streamtube. The sum of these breakthrough curves gives the cumulative streamtube result given by the RST method. For this case, the individual streamtube curves breakthrough at similar times due to the uniform velocity profile. As would be expected for this case, the CDR and RST method produce almost 
identical results. Table 5-4 compares the CPU times of the methods. RST is considerably faster

\begin{tabular}{|l|l|}
\hline \multicolumn{1}{|c|}{ Parameter } & \multicolumn{1}{c|}{ CPU Time (sec) } \\
\hline \hline CDR (4225 node problem) & 1508 \\
\hline RST (8 streamlines) & 125 \\
\hline
\end{tabular}

Table 5-4. Comparison of CPU times between CDR and RST for low infiltration rate case with nonreactive tracer

for this problem compared to some of the verification problems in Chapter 4 because only 8 streamlines are required to match CDR due to the homogeneous nature of the flow field.

The simulations are more interesting for the $4 \mathrm{~mm} / \mathrm{yr}$ infiltration scenario. At this higher infiltration rate, the zeolitic and nonzeolitic regions begin to differ in relative permeability. The flow begins to bypass highly zeolitized portions of the block (Figure 5-11) resulting in some preferential pathways in the velocity profile. This effect will be amplified in the ${ }^{237} \mathrm{~Np}$ reactive transport simulations since the highly zeolitized areas retard ${ }^{237} \mathrm{~Np}$ whereas nonzeolitized regions do not. Figure 5-12 shows that the streamtubes for the $4 \mathrm{~mm} / \mathrm{yr}$ infiltration scenario contract and expand with depth much more than for the $0.1 \mathrm{~mm} / \mathrm{yr}$ infiltration rate. The figure also shows that the breakthrough curves from the individual streamtubes are no longer breakthrough at the similar times. However, the cumulative breakthrough curve RST result matches the CDR result quite well. 

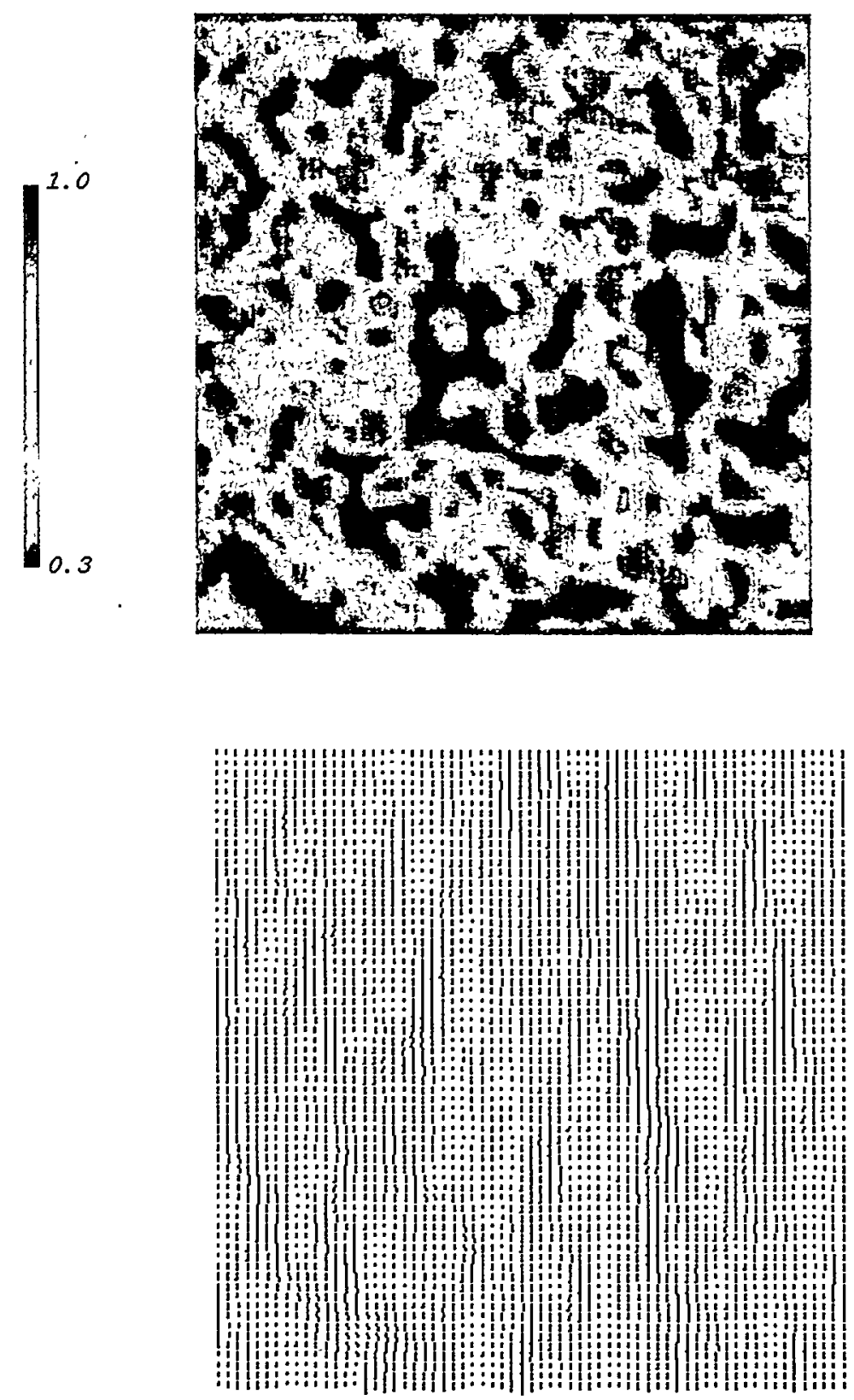

Figure 5-9.(a) The saturation profile and (b) the velocity profile for the low saturation case. The infiltration rate across the block is approximately $0.1 \mathrm{~mm} / \mathrm{yr}$ 

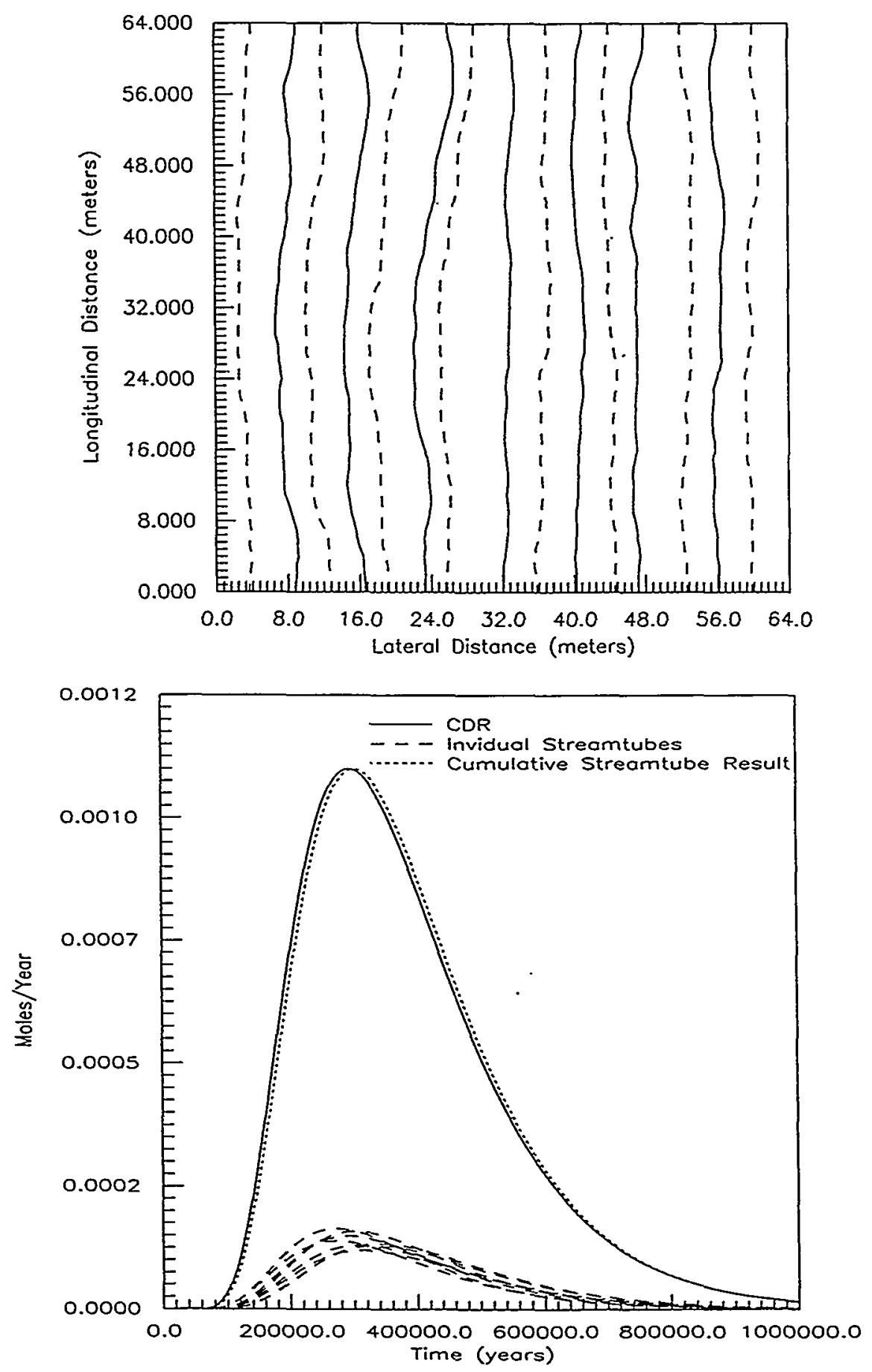

Figure 5-10.(a) The flow field is decomposed into eight equal flow streamtubes for the low saturation case. The solid lines represent the edges of the streamtubes and the dashed lines represent the center of the streamtubes, (b) the flux exiting the bottom boundary of the block. 

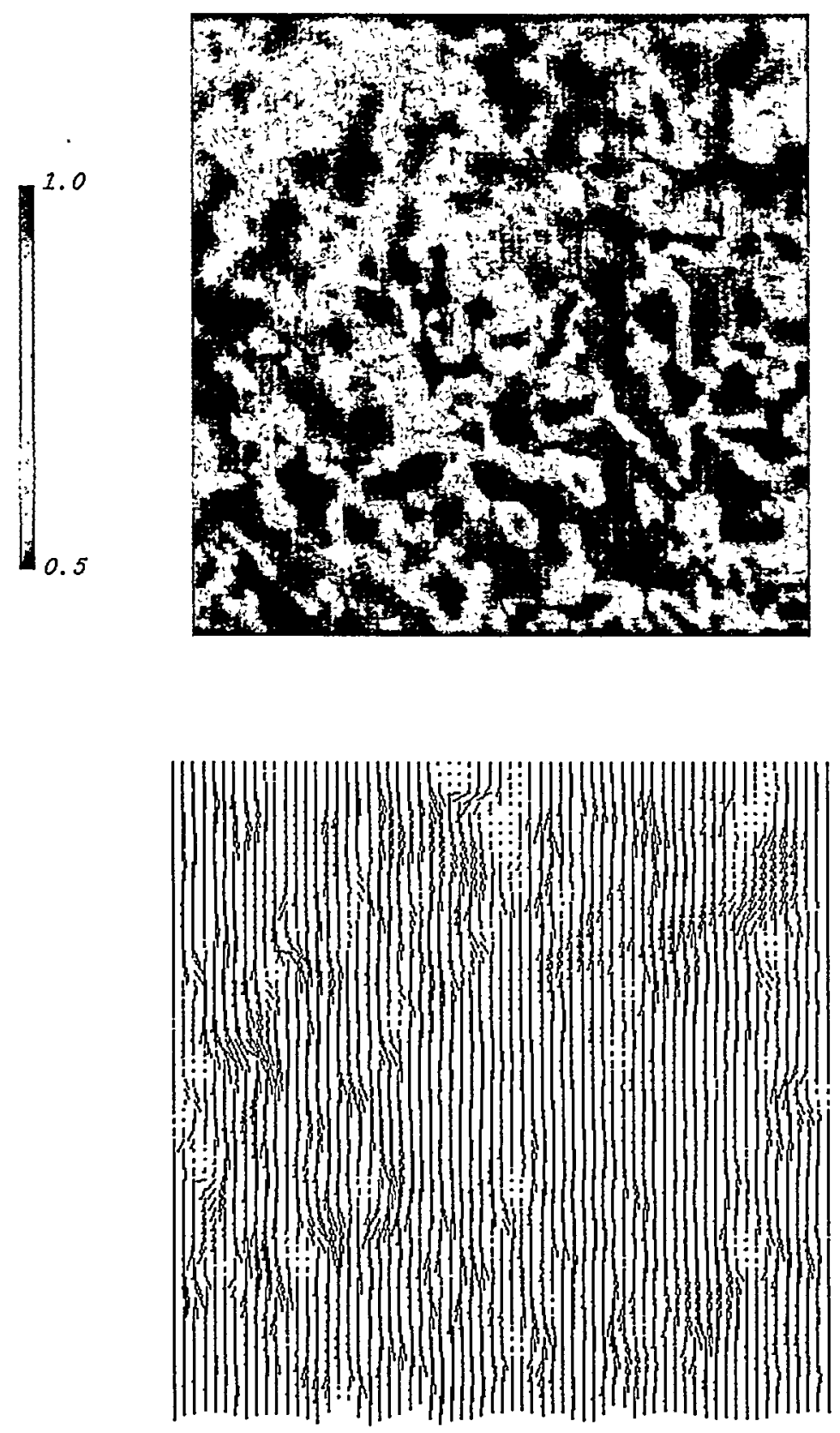

Figure 5-11.(a) The saturation profile and (b) the velocity profile for the high saturation case. The infiltration rate across the block is approximately $4 \mathrm{~mm} / \mathrm{yr}$. 

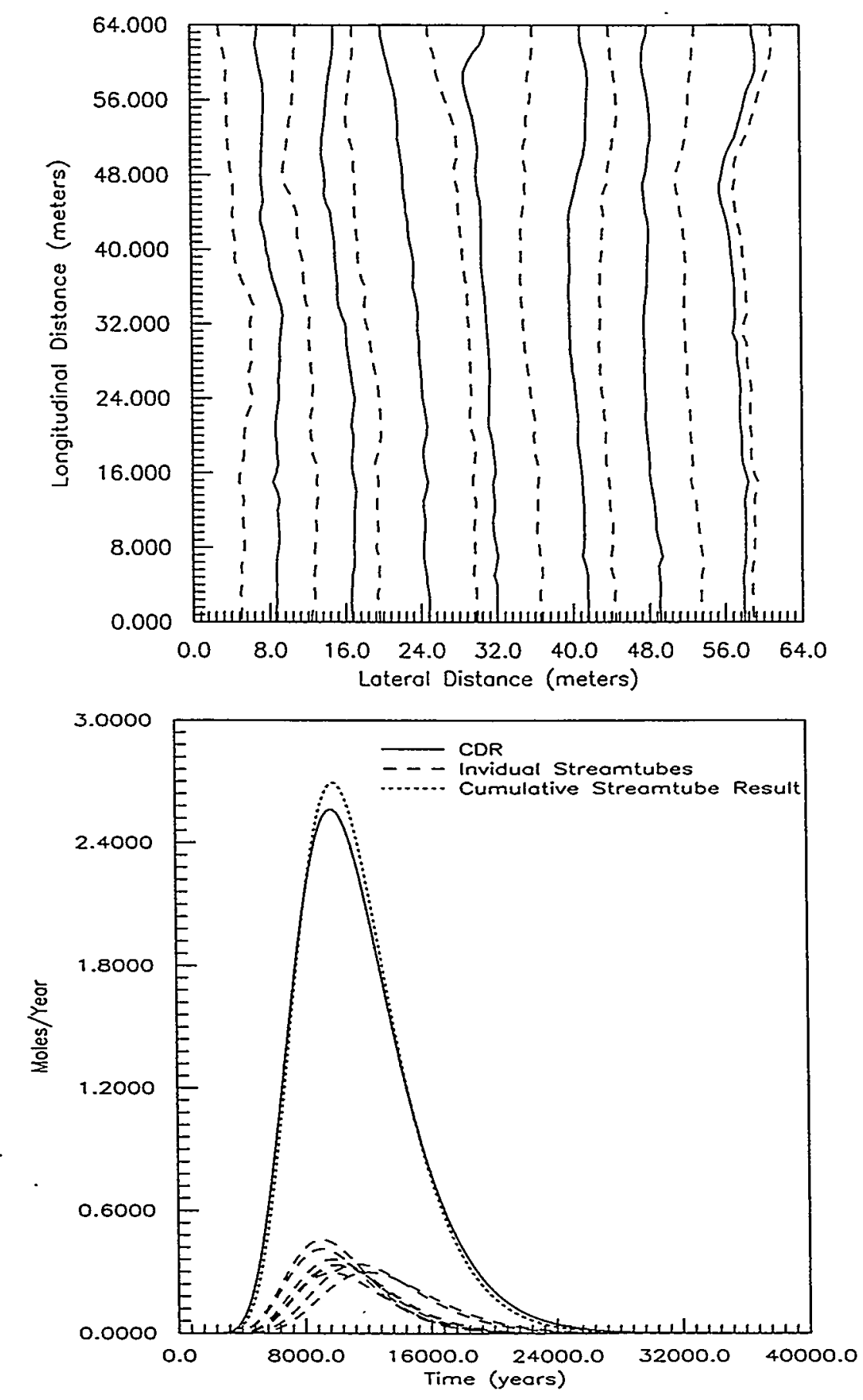

Figure 5-12.(a) The flow field is decomposed into eight equal flow streamtubes for the high saturation case. The solid lines represent the edges of the streamtubes and the dashed lines represent the center of the streamtubes, (b) the flux exiting the bottom boundary of the block. 
The CDR result exhibits slightly more dispersion than the RST result. Table 5-5 compares the CDR and RST results and shows a very similar trend to the $0.1 \mathrm{~mm} / \mathrm{yr}$ CPU times.

\begin{tabular}{|l|l|}
\hline \multicolumn{1}{|c|}{ Parameter } & \multicolumn{1}{c|}{ CPU Time (sec) } \\
\hline \hline CDR (4225 node problem) & 1224 \\
\hline RST (8 streamlines) & 110 \\
\hline
\end{tabular}

Table 5-5. Comparison of CPU times between CDR and RST for high infiltration rate case with nonreactive tracer

\subsubsection{Neptunium Transport Simulations}

In this section, we repeat the $0.1 \mathrm{~mm} / \mathrm{yr}$ and $4 \mathrm{~mm} / \mathrm{yr}$ infiltration scenarios with the reactive radionuclide ${ }^{237} \mathrm{~Np}$. Recall from previous chapters that the geochemical processes we consider are: aqueous speciation of ${ }^{237} \mathrm{~Np}$ into non-sorbing carbonate/hydroxy complexes and the sorbing $\mathrm{NpO}_{2}^{+}$cation, and sorption of $\mathrm{NpO}_{2}^{+}$via an ion exchange mechanism. We assume that ${ }^{237} \mathrm{~Np}$ released into the aqueous phase participates in speciation reactions. These speciation reactions are given in Table (Viswanathan et al., 1998). The ion-exchange reactions 3-4 and 3-5 are used to simulate the sorption of $\mathrm{NpO}_{2}^{+}$. In this example, we simulate the transport of ${ }^{237} \mathrm{~Np}$ through a random field of permeability. The concentration of $\mathrm{NpO}_{2}^{+}$sorption sites are correlated to the permeability at each location as we explain in section 5.4.3. Table 5-6 shows the parameters used in this simulation.Figure 5-13 compares the CDR and RST models for the $1 \mathrm{~mm} / \mathrm{yr}$ infiltration rate. The sorption of ${ }^{237} \mathrm{~Np}$ to the highly zeolitized portions of the Calico Hills results in a large tail in the breakthrough curve. In general, the entire breakthrough is greatly delayed when compared to the nonreactive transport case (see Figure 5-10) due to the ion exchange of ${ }^{237} \mathrm{~Np}$ with the zeolitic tuffs.Recall, that at this infiltration rate, the velocity field is very uniform so it is not surprising that 


\begin{tabular}{|l|l|}
\hline \multicolumn{1}{|c|}{ Parameter } & \multicolumn{1}{c|}{ Value } \\
\hline \hline Initial $\mathrm{pH}$ & 8.0 \\
\hline Injection $\mathrm{pH}$ & 7.0 \\
\hline Initial Total Carbonate Concentration & $2.52 \times 10^{-3} \mathrm{~mol} / \mathrm{L}$ \\
\hline Injection Concentration of Total Carbonate & $2.52 \times 10^{-3} \mathrm{~mol} / \mathrm{L}$ \\
\hline Initial Total ${ }^{237} \mathrm{~Np}$ Concentration & $0.0 \mathrm{~mol} / \mathrm{L}$ \\
\hline Injection Concentration of Total ${ }^{237} \mathrm{~Np}$ & $1 \times 10^{-5} \mathrm{~mol} / \mathrm{L}$ \\
\hline Time of pulse input of ${ }^{237} \mathrm{~Np}$ at top boundary & 10000 days \\
\hline Initial Total Sodium Concentration & $5.43 \times 10^{-3} \mathrm{~mol} / \mathrm{L}$ \\
\hline Injection Concentration of Total Sodium & $5.43 \times 10^{-3} \mathrm{~mol} / \mathrm{L}$ \\
\hline Initial Total Calcium Concentration & $2.91 \times 10^{-4} \mathrm{~mol} / \mathrm{L}$ \\
\hline Injection Concentration of Total Calcium & $2.91 \times 10^{-4} \mathrm{~mol} / \mathrm{L}$ \\
\hline Concentration of Sodium Sites & $\mathrm{spatially} \mathrm{variable}$ \\
\hline Concentration of Calcium Sites & $6.84 \times 10^{-5} \mathrm{~mol} / \mathrm{kg}$ rock \\
\hline
\end{tabular}

Table 5-6. Parameters for ${ }^{237} \mathrm{~Np}$ simulations

the CDR and RST methods agree very closely. Table 5-7 demonstrates that for these reactive transport simulations RST is about 14 times faster than the CDR method.

\begin{tabular}{|l|l|}
\hline \multicolumn{1}{|c|}{ Parameter } & \multicolumn{1}{c|}{ CPU Time (sec) } \\
\hline \hline CDR (4225 node problem) & 5787 \\
\hline RST (8 streamlines) & 410 \\
\hline
\end{tabular}

Table 5-7. Comparison of CPU times between CDR and RST for ${ }^{237} \mathrm{~Np}$ transport for low infiltration rate case

RST is very efficient for this problem because the flow field for this low $0.1 \mathrm{~mm} / \mathrm{yr}$ infiltration rate is very uniform and eight one-dimensional reactive transport simulations run much faster than a 4225 node two-dimensional reactive transport problem. In general, the ratio between RST and CDR cpu times are similar for nonreactive and reactive transport problems, although the simula- 


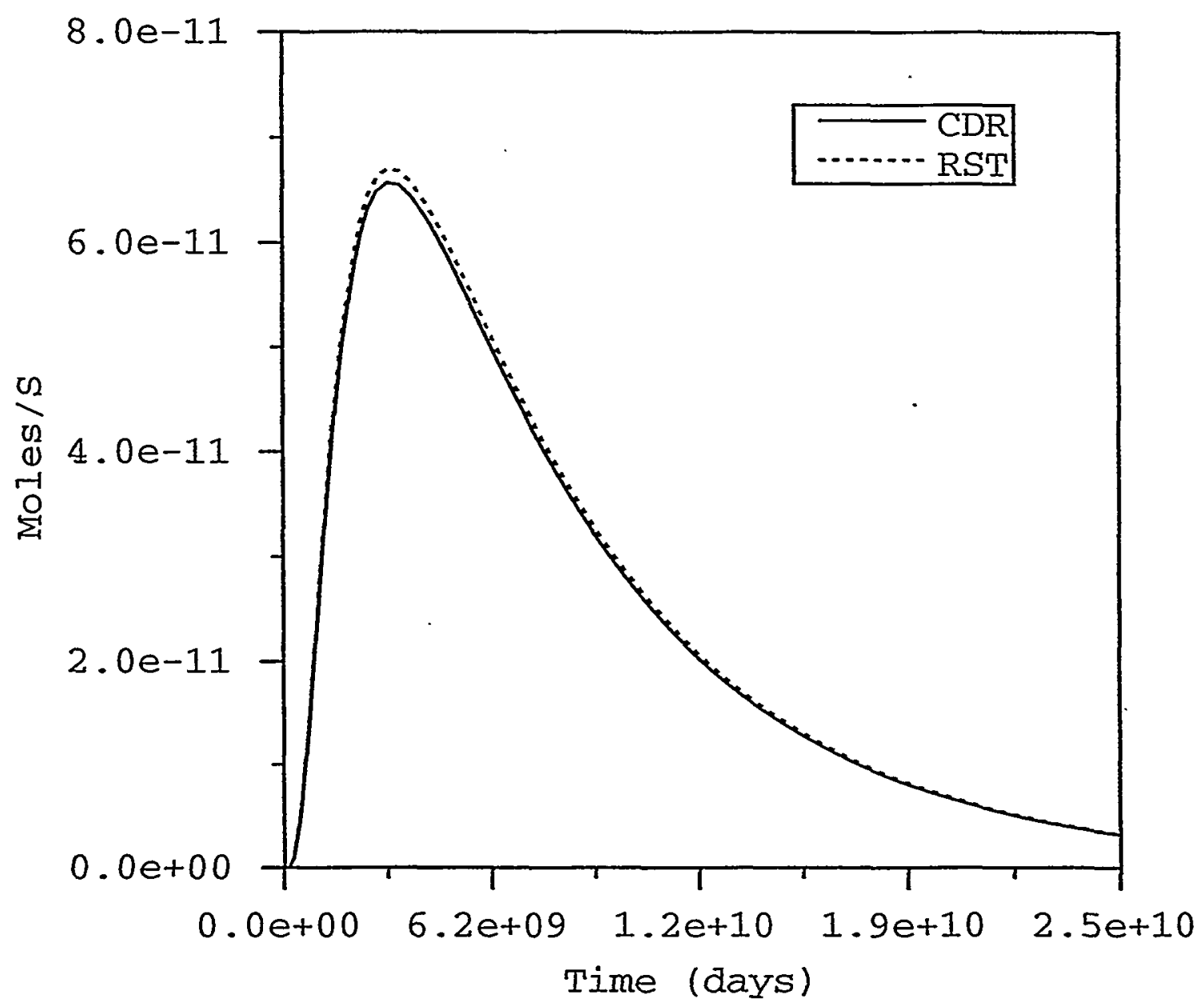

Figure 5-13. Comparison of the $\mathrm{RST}$ and $\mathrm{CDR}$ models for $0.1 \mathrm{~mm} / \mathrm{yr}$ infiltration rate. tions take longer since these reactive transport problems are more computationally intensive. For more complicated reactive transport problems we expect RST to increase in computational efficiency relative to the $\mathrm{CDR}$ method. Figure 5-14. compares the ${ }^{237} \mathrm{~Np}$ transport results for the $\mathrm{CDR}$ and RST models for the $4 \mathrm{~mm} / \mathrm{yr}$ infiltration rate scenario. The complex shape of the breakthrough curve is due to the combination of fast flow pathways that bypass the zeolitized portions of the Calico Hills and the slow flow pathways which pass through the zeolites retarding ${ }^{237} \mathrm{~Np}$. Unlike the other transport problems which required only eight streamlines to obtain a good match with the CDR method, this example required 25 streamlines due to the complexity of the flow field combined with the chemical reactions. RST matches the CDR method well for this complex problem 


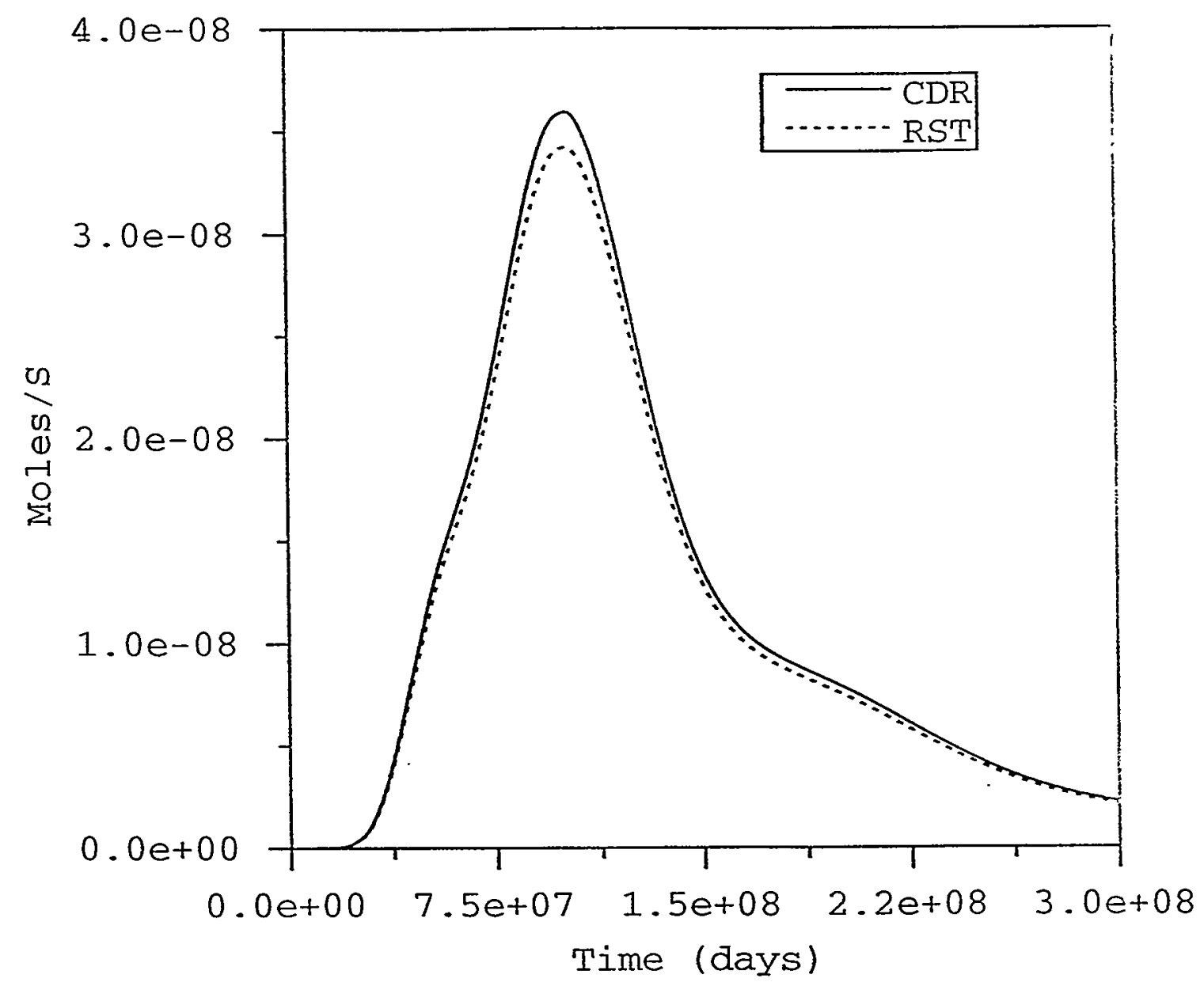

Figure 5-14. Comparison of the RST and CDR models for $4 \mathrm{~mm} / \mathrm{yr}$ infiltration rate.

in which saturation, permeability and the number of sorption sites are all spatially heterogeneous.Table 5-8 shows that due to the additional streamlines required by the RST method, RST is only about a factor of 4 faster than the CDR method.

\begin{tabular}{|l|l|}
\hline \multicolumn{1}{|c|}{ Parameter } & \multicolumn{1}{c|}{ CPU Time (sec) } \\
\hline \hline CDR (4225 node problem) & 5010 \\
\hline RST (25 streamlines) & 1244 \\
\hline
\end{tabular}

Table 5-8. Comparison of CPU times between CDR and RST for ${ }^{237} \mathrm{~Np}$ transport for high infiltration rate case 


\section{UNSATURATED ZONE TRANSPORT TEST}

\subsection{Summary}

The Unsaturated Zone Transport Test (UZTT) at Busted Butte provides a unique opportunity to compare the CDR and RST models. The UZTT at Busted Butte was designed to validate and continuously improve the Yucca Mountain Project's flow and transport site-scale model for the unsaturated zone. The UZTT at Busted Butte is located approximately five miles southeast of the potential repository site at Yucca Mountain, NV and contains an exposure of a distal extension of the Calico Hills formation, a major barrier to radionuclide migration in the unsaturated zone. The test consists of three phases. We have modeled the Phase 2 test block (10 m by $10 \mathrm{~m}$ by $7 \mathrm{~m}$ ) for which injection began on July 23, 1998 .

We utilize both the CDR and RST models to make predictions of the arrival times of tracers at specific locations in the test block. These predictions are compared to the most recent field results. The predictions utilize existing Yucca Mountain databases and provide a means of checking the models and the databases. These predictions are intended to test the current modeling concepts and tools available to the Integrated Site Scale model and they provide an assessment of that model (Robinson et al., 1997) for Performance Assessment. The predictions use parameters from currently available Yucca Mountain hydrologic and geochemical databases. At this stage, no model calibration has been performed.

At this time (July, 1999), only nonreactive breakthrough times at the collection boreholes are available from the test. We show that RST predicts breakthrough times at the collection plane that are similar to the CDR method with less computational expense. The fact that this is a transient flow problem reduces RST's computational efficiency. However, RST is still about three times as fast as the CDR method. Both the RST and CDR methods produce reasonable test results when compared to the field results indicating that the model parameters from the Yucca Mountain databases are in the correct range. 


\subsection{Background on Test}

The test itself is subdivided into 3 phases (Figure 6-1).Details of the test plan can be found

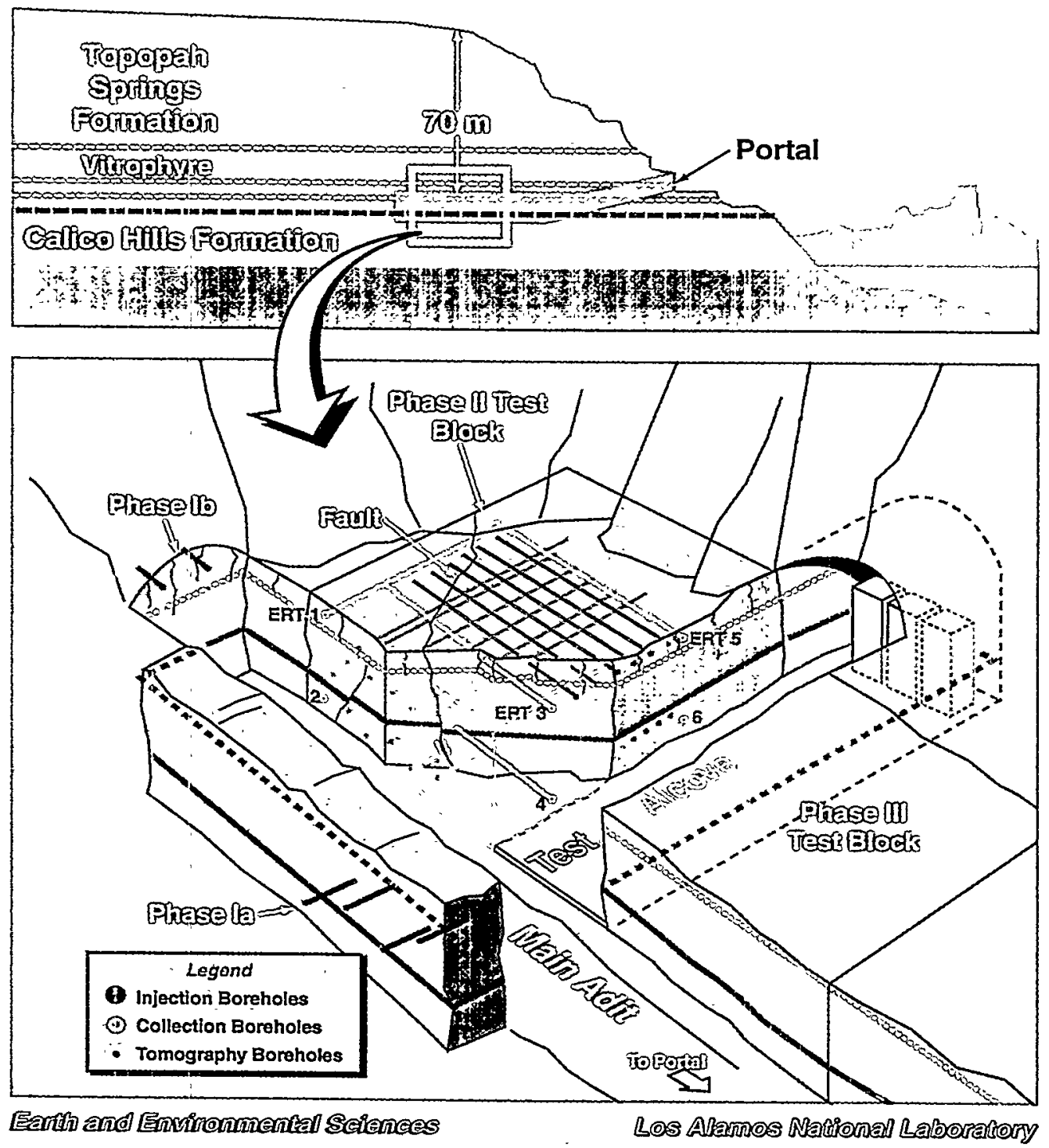

Figure 6-1. Schematic of the Busted Butte Phases. This schematic shows the locations of the different experiment phases and borehole locations.

in Bussod et al. (1997). Phases 1 and 2 are meant to provide tangible results by the end of 1999 and 
Phase 3 is planned for Performance Confirmation. Phase 1 involves six 2-meter deep single point injection boreholes and two inverted membrane collection boreholes. Phase 1 is meant as a scoping phase for Phases 2 and 3, as well as to provide testing results in early fiscal year 1999. Injection of a suite of nonreactive and reactive tracers was started on April 2, 1998 for Phase 1. The results from this testing phase were obtained from a mineback (see Figure 6-1 Phase 1A) and overcoring (see Figure 6-1 Phase 1B). Phase 2 represents an upscaled version of Phase 1 with 8 injection boreholes 7.5-8.0 meters in length and each containing 10 injection points. The injection points are distributed in 2 horizontal, subparallel planes arranged to test the properties of the lower Topopah. Springs Basal Vitrophyre and the hydrologic Calico Hills. All injection boreholes are located in the Test Alcove. The 12 collection boreholes in the Main Adit are 8.5-10.0 meters in length and each contain 15 collection pads evenly distributed on inverted membranes. Injection into Phase 2 occurred during the week of July 20, 1998.

We used FEHM in the development of the three-dimensional model to model Phase 2. Specifically, this code is used by the Yucca Mountain Project for radionuclide migration predictions using the "calibrated" site scale flow solutions (Bodvarsson et al., 1997) and therefore is ideal for predictions of the UZTT results. In effect, we are modeling a portion of the Calico Hills unit as we did in Chapter 5. The UZTT test block is not highly zeolitized like the cross sections we considered in the previous chapter. Therefore, we do not use zeolitic abundance to distribute the various properties in the block. Instead we rely on permeability measurements. Three-dimensional effects will probably become important as data specific to the test block become available for the Phase 2 block. The model was developed in three dimensions to capture these effects and to anticipate the three dimensional property database that will be collected for the test block.

Our first goal was to predict Phase 2 nonreactive and reactive tracer breakthrough times for each of the sampling boreholes for up to one year from the start of injection, the planned duration of the test. We only present nonreactive predictions in this thesis since reactive breakthrough results are not available yet from the test. Bussod et al. (1998) documents a more extensive set of simulations that includes predicted breakthrough times for reactive tracers. The CDR predictions 
in this thesis were made before the injection started, whereas, the RST results are more recent. However, the RST predictions produce very similar results since they use the same parameters as the CDR results. In effect both the CDR nd RST predictions can be considered "blind" predictions. The key difference being that RST is computationally faster than the CDR method but does not provide a three-dimensional representation of the plume as a function of time.

\subsection{Model Description}

The Phase 2 test block at Busted Butte encompasses from top to bottom the lower section of the Topopah Springs vitrophyre (Tptpv2) and the hydrologic Calico Hills unit (Tptpv1 and Tac) (Figure 6-1). The first step in constructing a three-dimensional finite element model of the Phase 2 test is to build a finite element mesh using the coordinates of the injection and collection boreholes (Bussod et al., 1998). Figure 6-2 shows a representation of the Phase 2 block with the

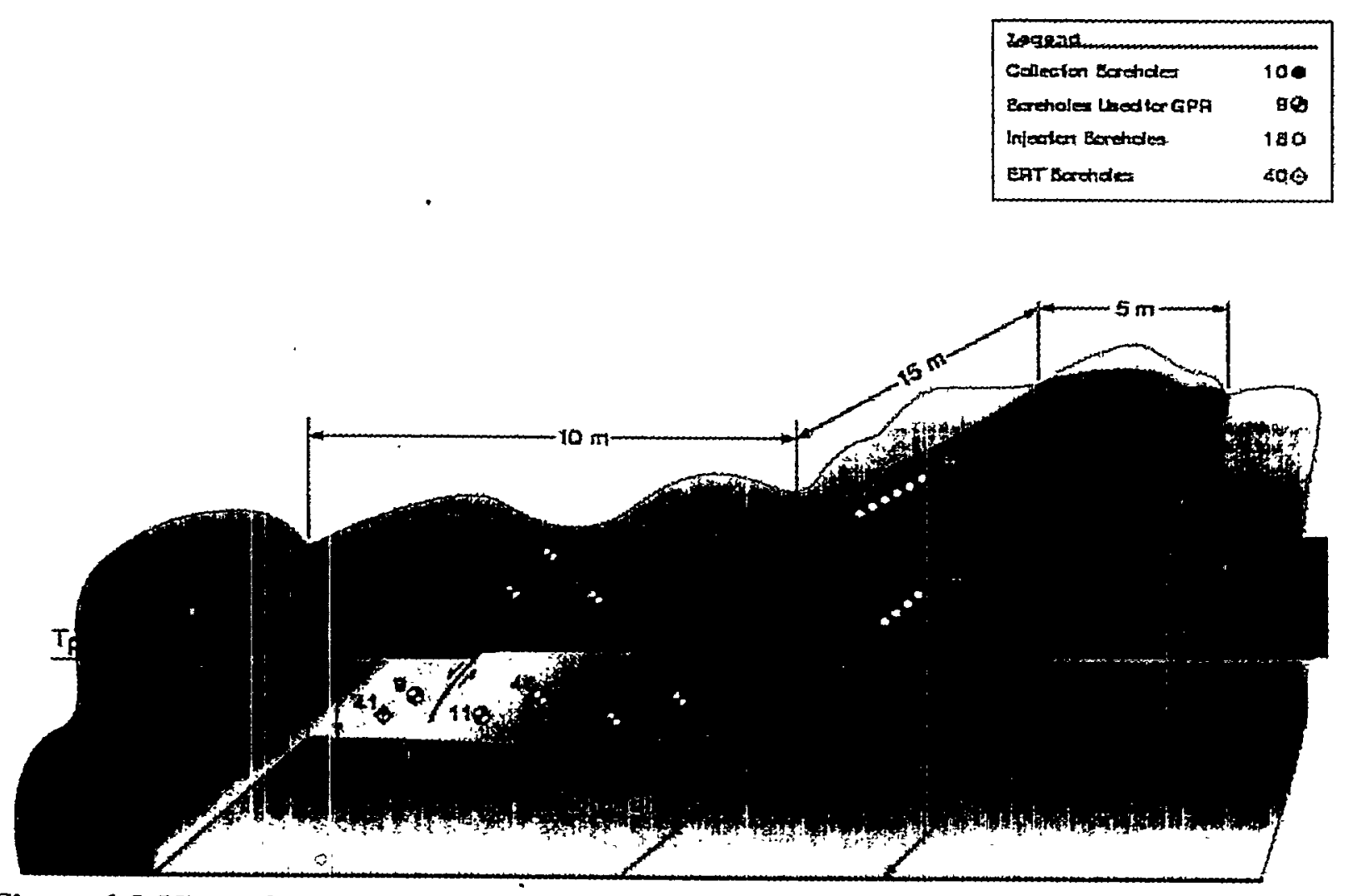

Figure 6-2. View of Phase 2 injection and collection boreholes 
location of the injection, collection, electrical resistance tomography (ERT) and groundpenetrating-radar (GPR) boreholes. The block is approximately 7 meters high $\mathrm{x} 15$ meters deep $\mathrm{x}$ 10 meters wide and contains twenty eight boreholes ranging from $7.5 \mathrm{~m}$ to $10.0 \mathrm{~m}$ in length. The eight subparallel injection boreholes are all located in the test alcove (Figure 6-2), distributed along two horizontal planes and are perpendicular to the twelve collection boreholes located in the Main Adit (Figure 6-2). Six of the 28 boreholes are dedicated to ERT and two are dedicated to GPR.

For the purposes of modeling, we chose the model domain to extend from +2 to +14 meters in the $\mathrm{x}$-direction, +60 to +72 meters in the $\mathrm{y}$-direction, and -8.2845 to +2.5015 meters in the $\mathrm{z}$ direction. These coordinates are consistent with the surveyed local coordinates and the stratigraphy of the block. Figure 6-3 shows a top view of the finite element grid with the borehole locations.

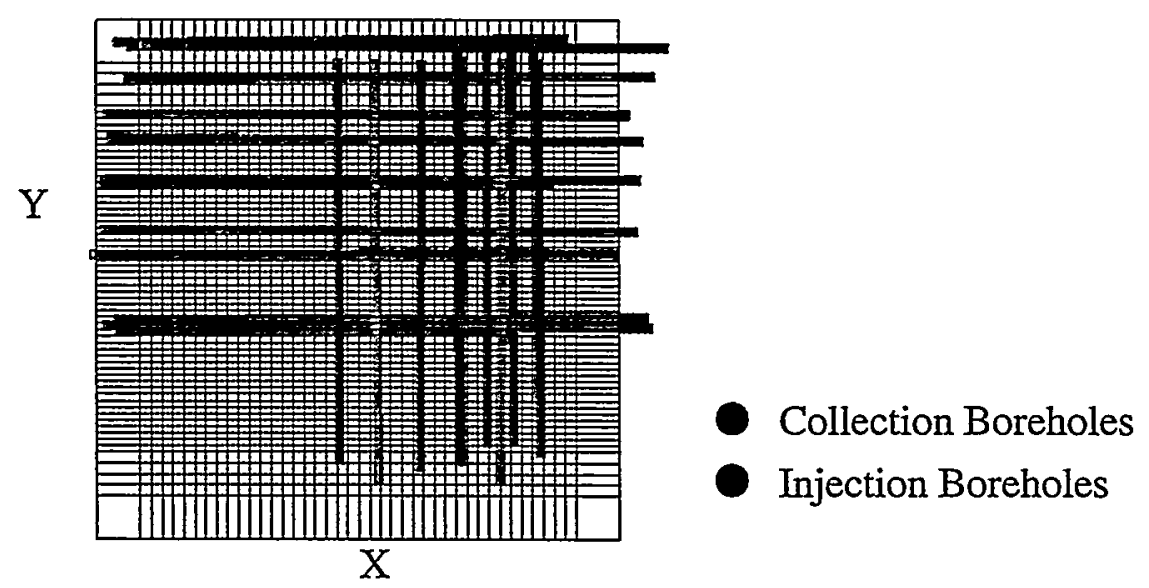

Figure 6-3. Top view of finite element grid, injection and collection boreholes.

In general, the mesh was refined at locations between the injection and collection boreholes to accurately capture the migration of the tracers and heterogeneities at scales smaller than the layer thickness. In the $\mathrm{x}$-direction, we chose a grid spacing of 0.25 meters at locations close to the boreholes. In both the $\mathrm{x}$ and $\mathrm{y}$ directions we chose a coarse mesh spacing at the block boundaries, since no transport is expected at these locations. In the y-direction, we chose a mesh spacing of $0.125 \mathrm{~m}$ at locations close to boreholes. A slightly finer grid spacing was used in the $\mathrm{y}$-direction than the $\mathrm{x}$-direction in order to accurately capture the location of the injection points, which are spaced 0.61 meters apart in the y-direction (10 injection points per injection borehole). In the z- 
direction, we represent the stratigraphy with six distinct layers: 5 layers to represent the hydrologic Calico Hills unit (Tac: 3. layers; Tptpv1: 2 layers), and 1 layer to represent Tptpv2. The discretization in the $\mathrm{z}$-direction is dependent on the particular layer since some layers are thicker than others. The discretization ranged from 0.15 to 0.25 meters. The entire model is comprised of 128,570 nodes. Figures $6-4$ and 6-5 show views of the grid from the Test Alcove and the Main

(3) Collection Boreholes

Injection Boreholes

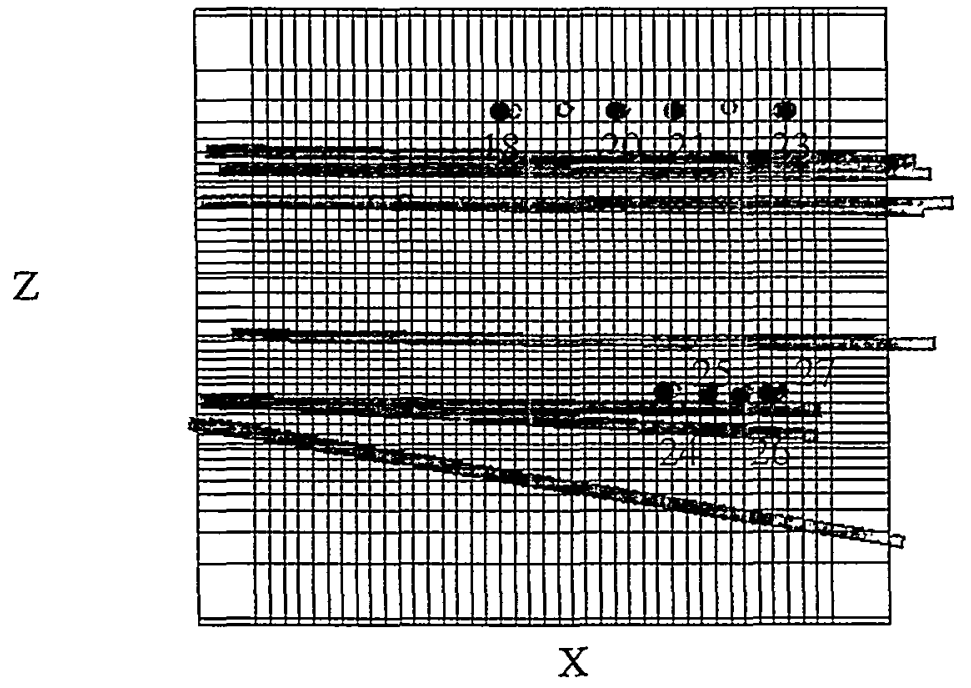

Figure 6-4. The finite element grid with injection boreholes as seen from the Test Alcove. The colored lines represent the location of collection boreholes which are perpendicular to the injection boreholes.

Adit, respectively. Once the mesh was constructed, the next step was to assign properties to the model.

For this preliminary investigation, layers 1 through 3 were lumped together as one Calico Hills unit (Tac), layers 4-5 were assigned Tptpv1 properties, and layer 6 was assigned Tptpv2 properties. As additional data become available, layers 1 through 3 , and 4 and 5 will all be treated as distinct layers. Porosity values were obtained from a few samples from the Busted Butte site (Bussod et al., 1998). Permeabilities and van Genuchten relative permeability parameters for the matrix were obtained from Flint et. al (1998), which was chosen because it represents the existing YMP database 
- Collection Boreholes

- Injection Boreholes

$\mathrm{Z}$

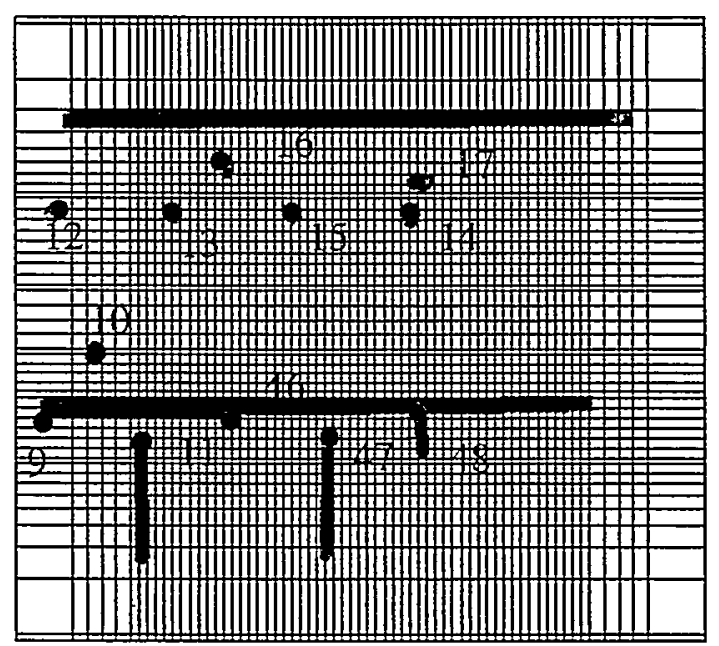

$\mathrm{Y}$

Figure 6-5. The finite element grid with collection boreholes as seen from the Main Adit. The horizontal colored lines represent the location of injection boreholes which are perpendicular to the collection boreholes. The vertical colored line represents plunging collection boreholes.

for the unsaturated zone and it contained sufficient samples to generate statistics on the variability of key parameters, such as matrix permeability and van Genuchten parameters. Ideally, a database of fracture properties for the Busted Butte site would be used, but these data are not yet available. Instead, fracture van Genuchten parameters were taken from the "calibrated" flow model of Bodvarsson et al. (1997). These data were obtained by fitting field data at the site scale. Although there is a great amount of uncertainty in fracture properties, we consider this data set to be a reasonable representation of YMP material properties applicable to Busted Butte. For this thesis, in view of the absence of data on fracture-matrix interactions in the Calico Hills, we have used the equivalent continuum model (ECM) to model Phase 2. Specifically, the relative permeability curves for the fractured rock are assumed to be adequately represented by an equivalent continuum model (ECM). The primary assumption in the model is that the capillary pressure in the fracture medium equals that in the matrix. Assuming that fracture and matrix domains can each be described using the characteristic curve model introduced by van Genuchten (1980), the composite permeability of 
the medium at a given matrix saturation (and capillary pressure) can be represented as a weighted average of the permeabilities of the two media. With this assumption, a single value can be obtained for the relative permeability. Table 6-1 contains the property sets used in the different layers for van Gnuchten model.

\begin{tabular}{|c|c|c|c|c|c|c|c|c|c|c|}
\hline \multirow[b]{2}{*}{$\begin{array}{l}\text { Busted } \\
\text { Butte } \\
\text { Layer }\end{array}$} & \multicolumn{4}{|c|}{ Flint(1998) } & \multicolumn{5}{|c|}{ Bodvarsson et al. (1997) } & \multirow{2}{*}{$\begin{array}{l}\text { Bussod } \\
(1998)\end{array}$} \\
\hline & Layer & $\begin{array}{l}\alpha_{\mathrm{m}} \\
(1 / \mathrm{m})\end{array}$ & $\mathrm{n}_{\mathrm{m}}$ & $\begin{array}{l}k_{m_{2}} \\
\left(m^{2}\right)\end{array}$ & Layer & $\begin{array}{l}\alpha_{\mathrm{f}} \\
(1 / \mathrm{m})\end{array}$ & $\mathrm{n}_{\mathrm{f}}$ & $\begin{array}{l}k_{f} \\
\left(m^{2}\right)\end{array}$ & $\phi_{\mathrm{f}}$ & \\
\hline 1. Tacl & $\mathrm{CHV}$ & 3.5 & 1.19 & $5 e-12$ & $\mathrm{CH} 1 \mathrm{v}$ & 11.52 & 3.0 & $2.43 e-9$ & $7.14 \mathrm{e}-5$ & 0.5 \\
\hline 2. Tac2 & $\mathrm{CHv}$ & 3.5 & 1.19 & $5 e-12$ & $\mathrm{CH} 1 \mathrm{v}$ & 11.52 & 3.0 & $2.43 \mathrm{e}-9$ & $7.14 \mathrm{e}-5$ & 0.5 \\
\hline 3. Tac3 & $\mathrm{CHv}$ & 3.5 & 1.19 & $5 e-12$ & $\mathrm{CH} 1 \mathrm{v}$ & 11.52 & 3.0 & $2.43 \mathrm{e}-9$ & $7.14 \mathrm{e}-5$ & 0.5 \\
\hline 4. Tptpv1 & BT1 & 0.56 & 1.31 & $1 . e-13$ & $\mathrm{CH}$ & 11.52 & 3.0 & $2.43 \mathrm{e}-9$ & $7.14 \mathrm{e}-5$ & 0.5 \\
\hline 5. Tptpv1 & $\mathrm{BT} 1$ & 0.56 & 1.31 & 1.e-13 & $\mathrm{CH} 1 \mathrm{v}$ & 11.52 & 3.0 & $2.43 e-9$ & $7.14 \mathrm{e}-5$ & 0.5 \\
\hline 6. Tptpv2 & PV2 & 2.2 & 1.25 & $1 e-16$ & TSW2 & 0.91 & 2.92 & $6.6 e-9$ & $1.29 \mathrm{e}-4$ & 0.25 \\
\hline \multicolumn{11}{|c|}{$\begin{array}{l}\alpha_{m} \text { van Genuchten alpha parameter for matrix material } \\
n_{m} \text { van Genuchten } n \text { parameter for matrix material } \\
k_{m} \text { matrix permeability } \\
\alpha_{f} \text { van Genuchten alpha parameter for fracture material } \\
n_{m} \text { van Genuchten } n \text { parameter for fracture material } \\
k_{f} \text { fracture permeability } \\
\phi_{f} \text { fracture volume fraction } \\
\theta \text { porosity }\end{array}$} \\
\hline
\end{tabular}

Table 6-1. Property sets for Phase 2 test.

As we refine our predictions, isotropic and anisotropic dual permeability models, and stochastic models will be utilized in addition to the ECM, if necessary.

The ECM was also used in scoping calculations done during the test designing and is currently being used to understand both Phases $1 \mathrm{~A}$ and 1B. Tracer breakthrough occurred in the $10 \mathrm{ml} / \mathrm{h}$ injection system of Phase $1 B$. Specifically, tracer was detected at the pad 35 days after injection. This places the time of breakthrough between 28 and 35 days corresponding to the collection pad schedule. In order to compare model predictions with test breakthrough times as defined by the appearance of fluorescein tracer on a collection pad, the concentration of the tracer must be known. For example, if we define the time of breakthrough to be when the normalized 
concentration reaches 0.5 , then for Tptpv 2 the ECM predicts a breakthrough at 47 days for a tracer to travel $28 \mathrm{~cm}$ (Figure 6-6).At a normalized concentration of 0.3 , the model predicts breakthrough
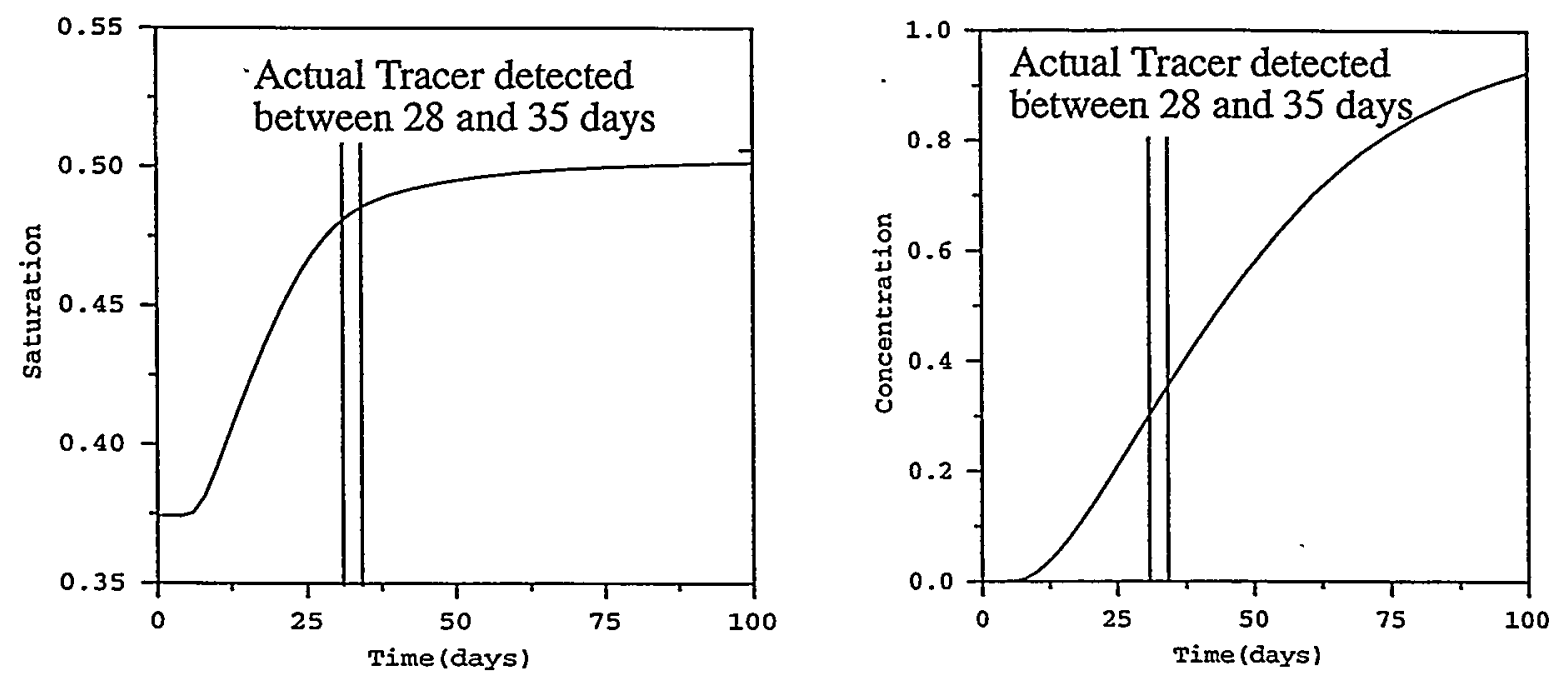

Figure 6-6. Using ECM and Tptpv2 properties to predict Phase 1B. The nonreactive breakthrough of tracer $28 \mathrm{~cm}$ from the injection pad: (a) saturation breakthrough, (b) concentration breakthrough.

at 31 days for the same distance, which is close to the observed breakthrough time for Borehole \#6. A mineback of the Phase 1B block provided another qualitative check to confirms that the model was approximating the real system. Specifically, the mine back of Phase 1B provided a 2D cross section of the fluorescein plume (Figure 6-7). Both the real system and the numerical simulation are dominated by capillary action with the plume migrating upwards as much as downwards. In addition, the plume spreads quite uniformly without much fingering.

For the first phase of predictive modeling and in the absence of appropriate data, we simply use the database listed in Table 6-1 with no attempt at calibration. The background flow conditions are obtained by setting equal capillary pressures at the top and bottom boundaries and allowing the block to equilibrate to a steady state saturation profile. A capillary pressure of $200 \mathrm{~m}$ of water was chosen, which is within the range of capillary pressures measurements at Yucca Mountain (Altman 


\section{Field Test Results}

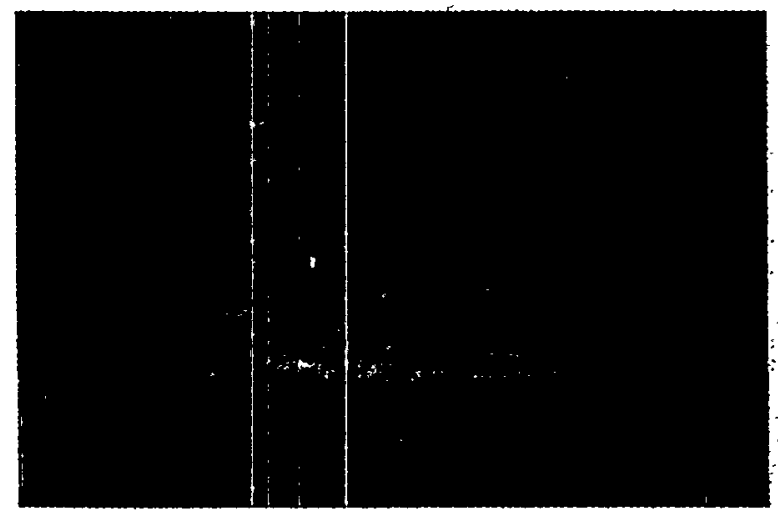

Numerical Simulation

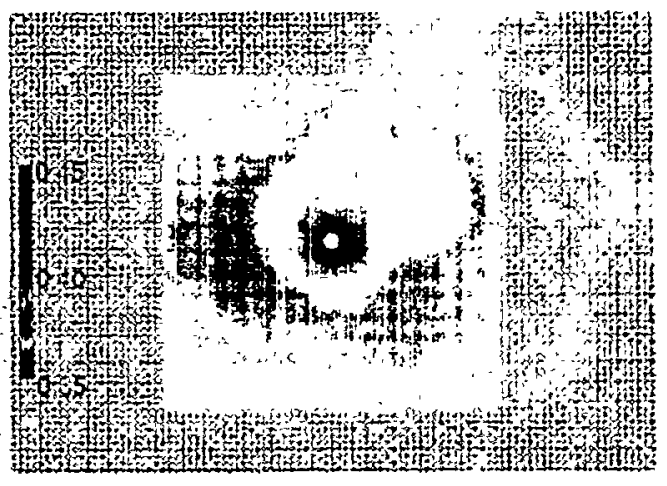

Fluid saturation during injection

Figure 6-7. Comparison of the actual fluorescein cross section from phase 1B mineback and numerical model. For further details see Bussod et al. (1998).

et al., 1996). The capillary pressure was set so that a saturation of about $0.35-0.45$ was obtained in the block. Moisture measurements and preliminary porosity data from test block lithologies, indicate that these saturation values are reasonable (Bussod et al., 1998). Once the background conditions are set, the next step is to begin pumping and injecting tracer.

Three different pumping rates are to be utilized in Phase 2: (a) $1 \mathrm{ml} / \mathrm{hr}$ ( 1 upper borehole), (b) $10 \mathrm{ml} / \mathrm{hr}$ (4 lower boreholes) and (c) $50 \mathrm{ml} / \mathrm{hr}$ ( 3 upper boreholes). The $1 \mathrm{ml} / \mathrm{hr}$ rate is equivalent to an infiltration rate of approximately $30 \mathrm{~mm} / \mathrm{yr}$ which is well within the range of infiltration rates at Yucca Mountain. The predictions we have made (shown below) show that during a 1 year test, the $1 \mathrm{ml} / \mathrm{hr}$ pumping rate is not expected to transport any tracer to the sampling wells. Injection borehole \#23 will be the only one that pumps at $1 \mathrm{ml} / \mathrm{hr}$ (Figure $6-2$ ). The $10 \mathrm{ml} / \mathrm{hr}$ injection rate is equivalent to an infiltration rate of approximately $380 \mathrm{~mm} / \mathrm{yr}$ which is slightly higher than the highest anticipated infiltration that will occur at Yucca Mountain. The lower injection boreholes $\# 24$, \#25, \#26, and \#27 will operate at $10 \mathrm{ml} / \mathrm{hr}$. Finally, $50 \mathrm{ml} / \mathrm{hr}$ is equivalent to an approximate infiltration rate of $1550 \mathrm{~mm} / \mathrm{yr}$. This infiltration rate is far higher than what is expected at Yucca Mountain even under wetter future climate scenarios. The purpose of the $50 \mathrm{ml} / \mathrm{hr}$ rate is to obtain 
enough separation in travel times between the nonreactive and reactive tracers so as to be visible and distinct in the field test. Boreholes \#18, \#20, and \#21 will pump continuously at $50 \mathrm{ml} / \mathrm{hr}$.

\subsection{Predictions}

The predictions below are borehole specific and can therefore be used to compare directly to test block results. We will only present CDR and RST results for the nonreactive tracer fluorescein as field results for the reactive tracers are not yet available. Table 6-2 shows the distance between the closest sampling point on the collection plane and the injection planes. These

\begin{tabular}{|l|l|l|l|}
\hline $\begin{array}{c}\text { Collection } \\
\text { Borehole }\end{array}$ & $\begin{array}{c}\text { Distance from top } \\
\text { injection plane (m) }\end{array}$ & $\begin{array}{c}\text { Collection } \\
\text { Borehole }\end{array}$ & $\begin{array}{c}\text { Distance from bottom } \\
\text { injection plane (m) }\end{array}$ \\
\hline \hline 16 & 0.61 & 46 & 0.175 \\
\hline 17 & 0.80 & 48 & 0.175 \\
\hline 14 & 1.17 & 9 & 0.175 \\
\hline 15 & 1.17 & 10 & 0.59 (above plane) \\
\hline 13 & 1.17 & & \\
\hline 12 & 1.17 & & \\
\hline
\end{tabular}

Table 6-2. Closest sampling point to the injection planes within each collection borehole

are the locations at which we predict the time of fluorescein breakthrough to compare to the actual test block measurements.

The use of RST with this problem is complicated by the fact that this is a transient flow problem. The streamlines can still be computed for this problem but we are limited by the time it takes for the flow solution calculations. Specifically, in all of the steady state problems, the time to obtain streamlines is very small compared to the time it takes to run multiple one-dimensional simulations. For these transient Busted Butte simulations, the time required to compute the 
streamlines makes up a significant portion of the total simulation. Table 6-3 compares the CPU

\begin{tabular}{|l|l|}
\hline \multicolumn{1}{|c|}{ Parameter } & CPU Time (sec) \\
\hline \hline $\begin{array}{l}\text { RST time to generate streamlines } \\
\text { due to transient flow }\end{array}$ & 3820 \\
\hline $\begin{array}{l}\text { RST time for running 7 one- } \\
\text { dimensional transport simulations } \\
\text { (1 for each collection borehole) }\end{array}$ & 100 \\
\hline Total RST simultation time & 3920 \\
\hline CDR & 11470 \\
\hline
\end{tabular}

Table 6-3. Comparison of CPU times between CDR and RST for simulation of UZTT

times between CDR and RST. RST is about 3 times faster than CDR because the CDR method takes several transport timesteps between each flow timestep, whereas, the time required for using the Pollack method to generate streamlines during the transient flow simulation is almost negligible.

With RST, we simply start a single streamline slightly below each injection point. We take the fastest arriving streamline to obtain travel times to the proper locations on the collection plane. With the CDR method, we monitor the closest sampling point to the injection plane in order to obtain travel times.For all predictions, we use three criteria for tracer breakthrough times: (a) a 5\% concentration limit, (b) a 50\% concentration limit, and (c) the concentration after 1 year from the time of injection. Note that we will assume that the concentration of tracer in the injection fluid is unity. Also note that tracers are continuously injected for the duration of the test.

We do not have a good idea for the values of longitudinal and transverse dispersivities. Dispersivity is a key parameter that affects the modeling results and must be determined with accuracy for future modeling efforts. For this preliminary investigation, we use a longitudinal and transverse (CDR only) dispersivities of $0.5 \mathrm{~m}$ and $0.05 \mathrm{~m}$ respectively. We use a diffusion coefficient of $1 \times 10^{-11} \mathrm{~m}^{2} / \mathrm{s}$. 
We predict the travel times for fluorescein, a nonreactive tracer. We explore the effects of the heterogeneity of properties within the layers. In these simulations, permeability values are . distributed within each layer. We assume that the mean of the permeability $\left(\mathrm{K}_{\text {sat }}\right.$ for matrix in Table 6-1) is the same as the permeability values used in the homogeneous simulations (see Viswanathan et al, 1998). In each layer, we assume a log normal distribution of permeability with a $\ln (k)$ variance of 2.0 and a correlation length of 1 meter in the $x, y$ and $z$ directions. Note that the test block is not highly zeolitized and therefore zeolitic abundance is not an important parameter in these simulations. In the Calico Hills and Tptpv1 units, the van Genuchten parameter, $\alpha_{m}$, has been shown to be correlated to matrix permeability (Altman et. al., 1995). We use this correlation as we did in Chapter 5 to distribute $\alpha_{\mathrm{m}}$ throughout the hydrologic Calico Hills or Tac and Tptpv1 units. In this thesis we present the results for just one realization. The methodology for performing Monte Carlo simulations now exists and the next step will be to run many more realizations.

Figure 6-8 shows the background saturation profile and the saturation profile after one year of continuous injection. The saturation profile after 1 year of injection shows that the $50 \mathrm{ml} / \mathrm{hr}$ boreholes have a strong effect on the saturation profile. This is for two reasons. The obvious reason is that $50 \mathrm{ml} / \mathrm{hr}$ is the high injection rate. The second reason is that the $50 \mathrm{ml} / \mathrm{hr}$ boreholes inject into the lower section Topopah Springs Basal Vitrophyre (Tptpv2) which has a much lower matrix permeability than the hydrologic Calico Hills (Tac and Tptpv1). The $10 \mathrm{ml} / \mathrm{hr}$ injections in the Tac unit does not have a large effect on the saturation profile. The simulations indicate that capillary action is an important process around the $10 \mathrm{ml} / \mathrm{hr}$ injection points. This is mostly due to the high matrix permeabilities in this unit.

Figure 6-9 depicts a CDR concentration plume for fluorescein after 1 year. Although, the permeability and the van Gneuchten $\alpha_{\mathrm{m}}$ are spatially variable, the concentration plume is uniform showing a system dominated by capillary forces. The two-dimensional cross-section presented in Figure 6-7 confirms that the uniform nature of the plume occurs in the actual system. Figure 6-10 shows streamlines from the injection boreholes obtained from the RST method. We start a single 

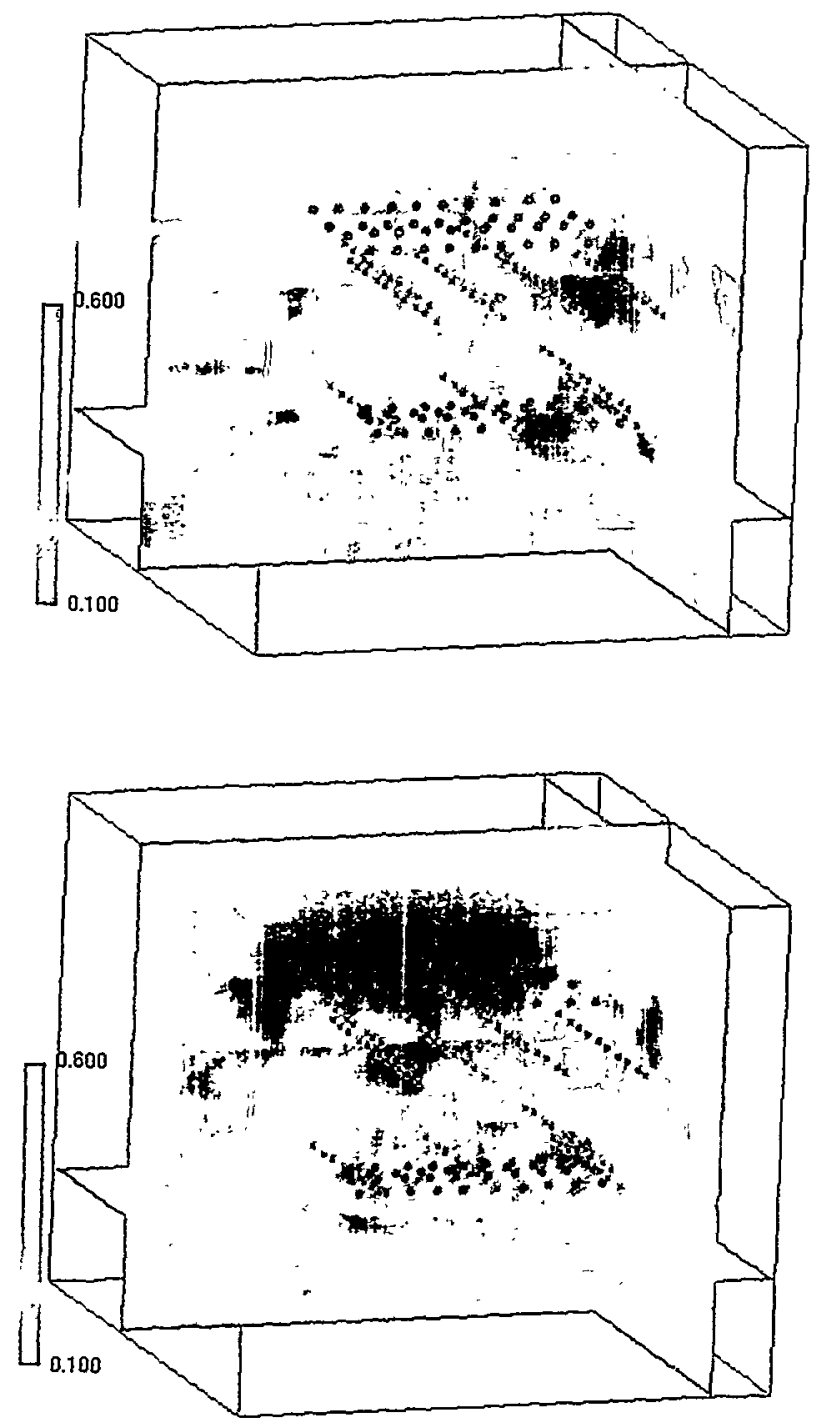

Injection points
Collection points

Figure 6-8. Saturation profile from 10 to $60 \%$ for a given realization: (a) background saturation, (b) saturation profile after 1 year of injection.

streamline slightly below each injection point. We take the fastest arriving streamlines to specified locations on the collection plane to obtain travel times from the injection plane to the collection plane. 


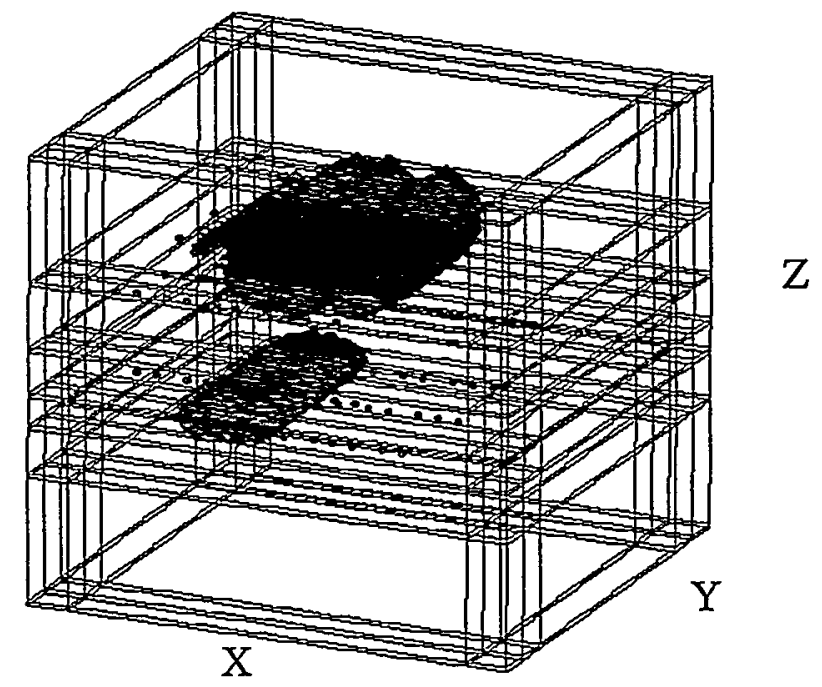

Figure 6-9. The concentration plume after 1 year of nonreactive tracer injection. The green isosurface represents a concentration of 0.5 . The red dots represent the sampling points along the collection boreholes.

Figures 6-11 and 6-12 compare the actual and predicted breakthrough times for the different collection boreholes. The CDR and RST results are so similar that we treat them as the same on the figures. Figure 6-11 shows that the actual measurements for boreholes 10, 13, 16,17 and 46 fall within the predicted 5\% and 50\% concentration breakthroughs. Borehole 10 is an impressive match as it is actually above the injection plane confirming that capillary forces are dominant in the system. Predicted breakthrough times for boreholes 9 and 48 are slower than the actual measurements indicating that there may be some preferential pathways in the actual system that are not accounted for in the model. Figure 6-12 shows that accuracy of the model is not dependent on the distance between the injection and collection points. One may expect the model results to increase in error as the distance increases. However, this is not the case because the collection boreholes with the most error are actually very close to the collection plane. This may be an indication of short preferential pathways such as fractures that facilitate the transport of fluorescein to boreholes 9 and 48. For the boreholes that are farther from the injection plane, any fracture flow may be sucked up by the matrix blocking preferential pathways. This may be why 

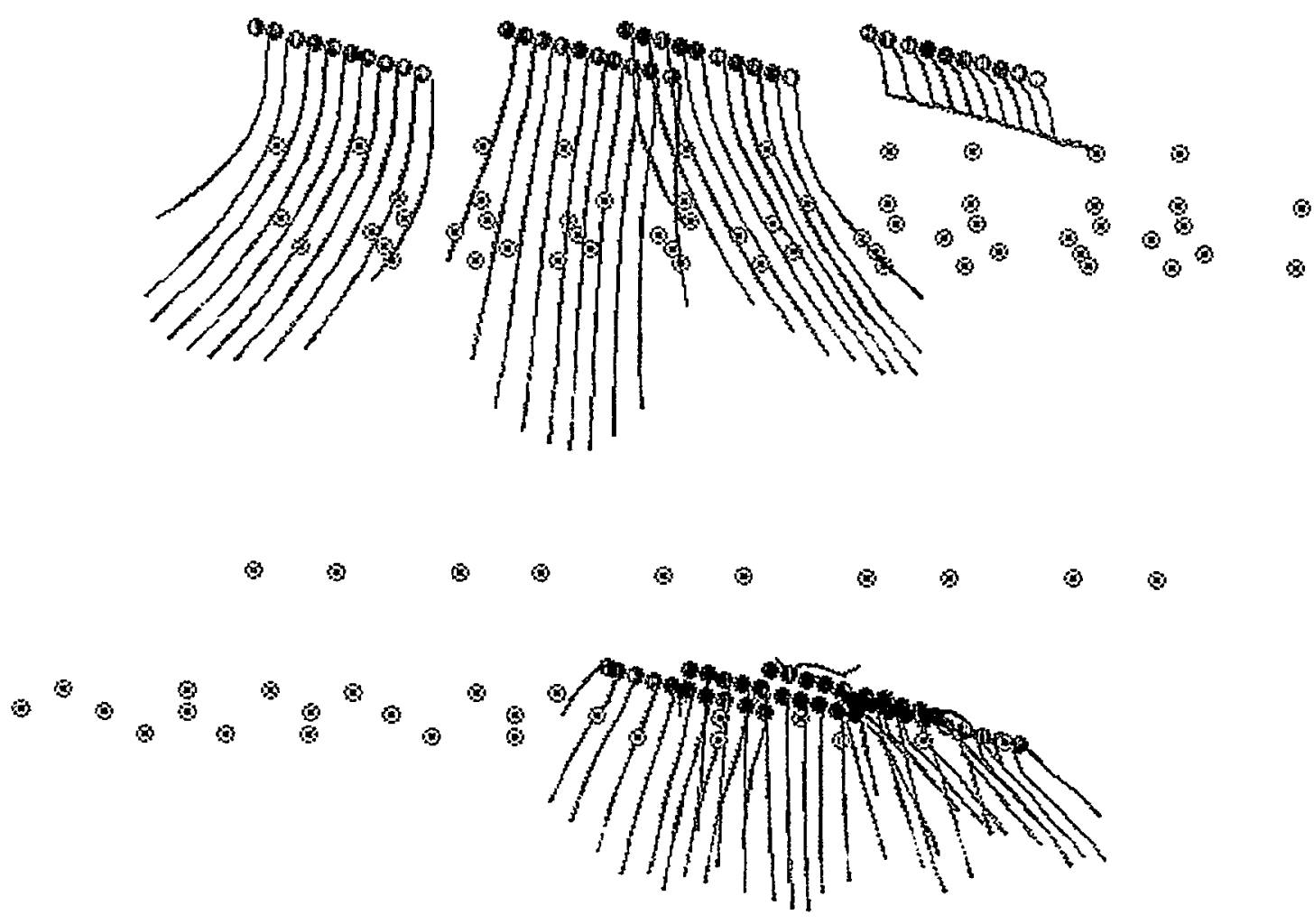

Figure 6-10. Streamlines from the injection point to 1 year after injection.

predicted breakthrough times for boreholes far from the injection plane are accurately predicted by the model. 


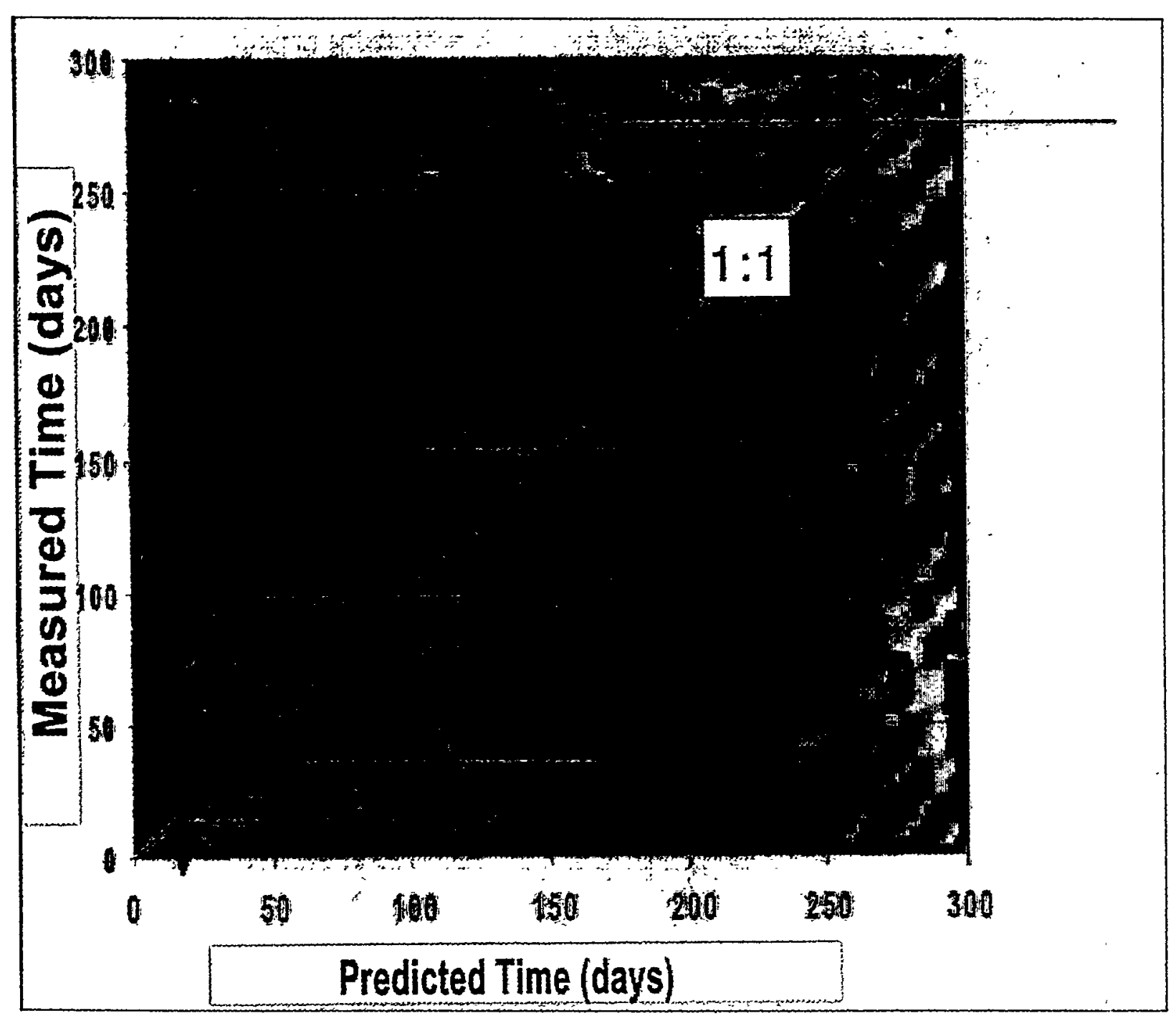

Figure 6-11. Comparison of Phase 2 modeling predictions to actual measured breakthrough times. The lower bound for the predicted time is the $5 \%$ concentration breakthrough and the upper bound is the $50 \%$ concentration breakthrough. Note that the measured breakthrough are from $1 \%$ to $45 \%$ concentration breakthrough. 


\section{Predicted vs. Measured Breakthrough}

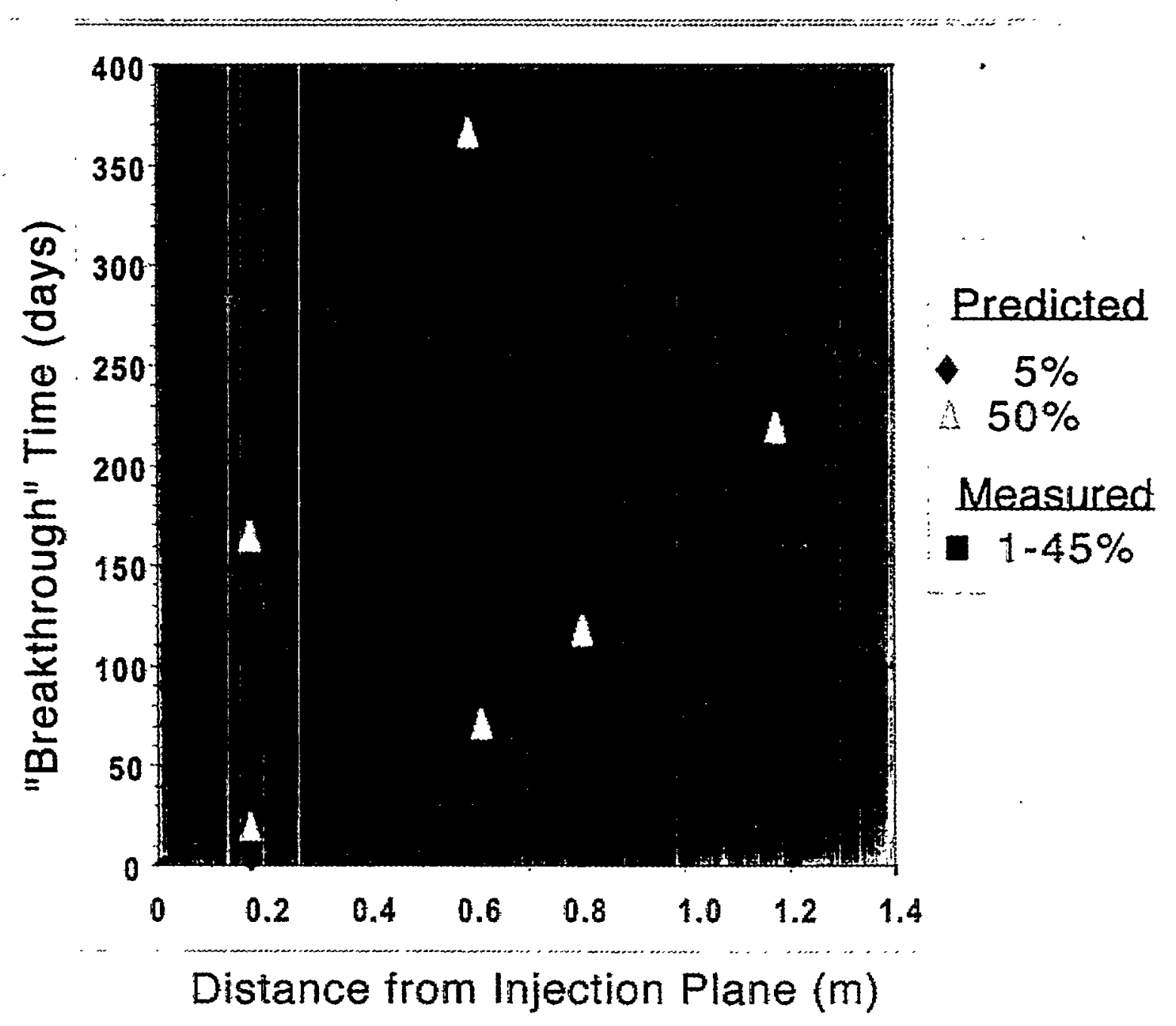

Figure 6-12..Comparison of measured and predicted breakthrough times as a function of distance from the injection plane. 


\section{CONCLUSIONS}

\subsection{Summary}

We have developed two reactive transport modeling techniques to simulate radionuclide transport at Yucca Mountain: 1) the selective coupling approach applied to the convectiondispersion-reaction (CDR) model and 2) a reactive streamtube approach (RST). The overall goal of the present study was to develop numerical techniques that can be used to solve the mixed equilibrium-kinetic transport problem in large, complex two- and three-dimensional domains. The goal was to develop flexible techniques that have wide applicability to a range of reactive transport systems. Specifically, the methods must handle both cpu time and memory usage efficiently. The techniques were developed in an existing finite element heat and mass transfer code called FEHM (Zyvoloski et al., 1997). FEHM is a three-dimensional heat and mass transport code simulator that can represent complex structures (e.g. faults and stratigraphy) and model non-linear processes such as vadose zone flow and heat flow. Our modifications to FEHM make it possible to model multicomponent reactive transport for these large two- and three-dimensional problem domains.

\subsection{Selective Coupling}

The selective coupling algorithm we have developed represents a versatile alternative to traditional SIA techniques and the global implicit method. We note first that the term "coupling" refers to the strategy for coupling the transport and reactions together, rather than the inherent coupled nature of the component concentrations.

The SIA technique attempts to solve the linearized algebraic equations for transport of the aqueous components one at a time. This approach, though simple to implement, is not always an efficient solution technique because it ignores the coupling between aqueous components linked 
through kinetic reactions. If the reactions that link the aqueous components are rapid, the SIA technique ignores important derivatives in the Jacobian matrix of the full system of equations.

The global implicit approach includes all derivatives, making no approximations in the formulation of the Jacobian matrix. Its performance is therefore controlled by the ability of the Newton-Raphson formulation to solve the system of nonlinear transport equations. This approach is likely to attain convergence efficiently for most cases. However, the global implicit method is very memory intensive for large problems with numerous aqueous components. The selective coupling method developed in the present study allows only the strongly coupled components to be solved together, and the transport iteration consists of solving groups of components simultaneously. This approach can result in computational savings relative to the global implicit method by achieving a similar total SIA iteration count while reducing the cpu time per iteration. More importantly, the memory requirements of the selective coupling technique are controlled by the maximum number of coupled components, rather than by the total number of components. For complex aqueous chemical systems and grids with a large number of nodes, the memory efficiency is the characteristic that makes the selective coupling method particularly attractive relative to the global implicit method.

There are many considerations to be made when developing a numerical technique for solving reactive transport problems. In our case, a flexible solution technique was desirable because the code was being developed for an existing, general-purpose finite element heat and mass transport code (FEHM). In the selective coupling method as implemented, the user selects the groups of solutes and the order the groups are solved. All possible coupling strategies, from the SIA-1 method to the global implicit technique, are available at run time. With respect to the solution of the coupled equations, the block matrix solver technology already used in the FEHM code used is ideally suited for this application. Equation solvers of this type are fairly common, and hence availability should not be a roadblock to implementing these techniques in other codes. 
Coupled normalization is another concept introduced in this paper for formulating the solution of the linearized equations resulting from the Newton-Raphson technique. Coupled normalization scales the equations in preparation for an iteration of the linear equations. Furthermore, we demonstrated how coupled normalization allows for the efficient coupling of aqueous and immobile components such that the computational and memory resources are dependent only on the number of coupled aqueous components; the immobile components are included with very low additional computational burden.

The choice of a chemical formulation with a combination of equilibrium and kinetic reactions results in a challenging system for obtaining an efficient numerical solution, but at the same time is a more versatile model formulation than a purely equilibrium-based system. Although it could be argued that the most computationally challenging problems for the SIA technique systems with rapid kinetic reactions - could be recast more efficiently as equilibrium reactions, there are several reasons that the more flexible system and the selective coupling solution procedure are desirable. First, geochemical rock-water reactions span an extremely large range of reaction rates, as do fluid velocities in porous media. Therefore, in practical applications a kinetic treatment is useful in many cases. Next, in many chemical systems there is scant knowledge on the kinetic parameters of the reactions. While no substitute for data, a sensitivity analysis in which kinetic parameters are varied over a wide range can provide insight into whether the lack of data are critical to understanding the system, or merely an uncertainty that is relatively unimportant to resolve. For this situation, the reactions in question are most conveniently cast as kinetic reactions; when large rate constants are selected the system behaves as an equilibrium system. This is preferable to having to recast the problem to handle the rapid-kinetics extreme. All of these reasons relate to the "robustness" of the numerical solution, which we define as the ability to obtain convergence for a wide range of input parameters in a reasonable time, without the need for intervention by the user once the problem is set up. Robustness is a particularly important attribute for a general purpose chemical transport model: if a solution cannot be obtained practically for some sets of parameters, then issues of cpu and memory efficiency are moot. 
Examination of the example problems presented here allows us to formulate general guidelines for the selection of a coupling strategy for practical problems. The following guidelines are supported by the example problems presented in Chapter 3 .

1. The most important aspect of the chemical system is the nature of the linking of aqueous component concentrations. Example 1 in Chapter 3 illustrated this concept by dealing only with aqueous components coupled through kinetic reactions. This example was actually a surrogate for more complex chemical systems with aqueous components linked indirectly through interphase kinetic reactions. The Damkohler numbers of the reactions strongly influenced which coupling strategy was the most appropriate.

2. Linking of aqueous components can also occur indirectly through competitive sorption reactions or dissolution/precipitation reactions, and must be considered in selecting the coupling strategy. Example 2 illustrated both the concept of the influence of kinetics and the indirect linking of aqueous components through competitive reactions. Specifically, we showed that selectively coupling $\mathrm{Co}^{2+}$ and $\mathrm{NTA}^{3-}$ greatly improved convergence. Although $\mathrm{Co}^{2+}$ and $\mathrm{NTA}^{3-}$ do not react with one another in a kinetic reaction, they were indirectly linked to one another due to the equilibrium speciation reactions. For this reason, coupling of the two components improved convergence.

3. The aqueous component concentrations themselves affect the nature of the chemical system and the optimum solution strategy. In Example 1, lowering the concentration of an aqueous component from a value at which it was in excess relative to the other concentrations to a value that was of the same order as the others made the solution more difficult to obtain without using a fully coupled strategy. The detailed knowledge required to optimally configure the solution strategy can perhaps be viewed as a limitation. However, it is just such an analysis that provides fundamental insight into the controlling factors of the behavior of the chemical system. Thus, we view the "burden" of gaining this insight as an advisable preliminary step in the analysis of a complex chemical transport system. In practice, this preliminary work can be carried out in simplified, steady-state, uniform flow and transport fields before progressing to more realistic scenarios. 
4. Coupled normalization results in a guaranteed memory savings when coupling immobile and mobile components, and hence should be employed whenever possible. In Example 3, the competitive ion exchange reaction presented a computationally demanding problem, especially for the more rapid kinetics. As rate constants increase, the most efficient strategy is to couple the three aqueous components and three immobile components. The coupled normalization approach couples the immobile components to the aqueous components in a way that effectively reduces the coupled solution from six to three degrees of freedom. The three aqueous components, with Jacobian derivative information from the three immobile components folded into the residual equations, were then solved as a system. The other components in this problem, $\mathrm{H}^{+}$and $\mathrm{HCO}_{3}^{-}$, were solved individually before the coupled solution step, thereby optimizing the computational and memory efficiency of the overall solution.

\section{The user should employ the minimum amount of coupling needed to solve a given problem effi-} ciently. The global implicit method is the most computationally demanding option on a per-iteration basis, and thus will underperform a strategy using a intermediate coupling, as long as the latter solves the equations in a similar number of iterations. Furthermore, for large systems, the memory demands associated with high degrees of coupling are potentially prohibitive. The memory requirement of the transport solution is governed by the maximum number of components coupled in the iterative scheme.

The flexibility of the techniques developed in the present study provides a great advantage in that the solution strategy can be tailored to the problem at hand. As reactive transport simulations begin to become more commonly performed for complex, multi-dimensional flow and transport systems, we believe that the numerical techniques developed in the present study should find widespread applicability in general purpose reactive transport codes.

A subject of future research could be to determine a method to automatically determine the coupling strategy from the specified reactions. Before begining the computation, an evaluation of the terms of the Jacobian matrix could provide the necessary information to determine the coupling 
strategy. However, the transient nature of reactive transport problems could make the evaluation difficult at the beginning of the simulation since the coupling terms may not be representative of the coupling terms later in the simulation. Another more complicated possibility would be to make the coupling strategy a function of time. Specifically, evaluate the Jacobian and determine the coupling strategy every time step. Automating the process, especially as a function of time, would require significant code development but would be an interesting topic of future research.

\subsection{Reactive Streamtube Model}

The reactive streamtube method (RST) we have developed is an alternative to solving large two- or three-dimensional reactive transport simulations with the CDR method for cases in which we are interested in calculating the average flux across a specific control plane. Calculating fluxes across control planes is a common method used in Performance Assessment models and therefore RST may be a useful tool for such assessments. In three-dimensional simulations, we showed that CDR method can result in large CPU times due to the fine grid spacing and small time steps required to avoid numerical dispersion errors and to obtain an accurate solution. Although iterative approaches such as selective coupling can lessen the computational expense, many problems are still too computationally intensive to solve. With RST, we replace one computationally intensive two- or three-dimensional CDR simulation with numerous computationally efficient one-

dimensional CDR simulations. The key assumption of RST is the neglect of transverse dispersion between streamtubes. Although mixing between streamtubes is neglected in the model, we argue that the apparent dispersion caused by complete mixing at the control plane is often greater than transverse dispersion between streamtubes.

We are interested in utilizing RST to simulate flow through heterogeneous domains since the technique can simulate sharp fronts and is computationally efficient. Performance Assessment models typically involve hundreds of Monte Carlo simulations in which numerous realizations are conducted in an attempt to capture the overall uncertainty of the system. If larger grid size are used 
to make the CDR model computationally feasible, then the resulting numerical dispersion will act to smooth out the breakthrough curve. Therefore, the CDR model tends to produce nonconservative estimates since it may under predict the peak concentration. For these reasons some of the large multidimensional CDR simulations have come under scrutiny by the Yucca Mountain External Review Committee (ref?). On the other hand, RST errs on the conservative side since RST neglects transverse dispersion and tends to over predict the peak concentration.

The examples in Chapter 4 show that neglecting transverse dispersion while mixing at the control plane is often valid since the CDR and RST methods usually produce similar average flux breakthrough curves at the collection plane. However there are exceptions in which transverse dispersion is one of the dominant processes in the system and cannot be neglected. For example in the vertical layer problem, we showed that if the time scale for transverse dispersion is less the transport time scale, RST produces erroneous results. This indicates that if there is a large discrepancy in travel times between adjacent streamlines, transverse dispersion may be an important process in the system. Another interesting result from this problem is that no matter how many streamlines are used, RST may not mimic transverse dispersion by simply mixing concentration fluxes at the control plane.

We have found that the accuracy and computational efficiency of RST is greatly dependent on the number of streamlines required to characterize the flow field. However, for the examples we considered RST was more efficient than the CDR method. For velocity fields that are not very heterogeneous (e.g. the $0.1 \mathrm{~mm} / \mathrm{yr}$ infiltration rate case discussed in sections 5.5.2 and 5.5.3), a small number of streamlines were required to match the CDR method, resulting in much greater efficiency for RST (factor of 14). For simulations with sharp changes in the velocity field (e.g. the 3D bypass problem discussed in section 4.3.2) numerous streamlines (e.g. 200) were required to characterize the flow field increasing the computational expense required to obtain an accurate result. For the two-dimensional random field problems (section 4.2.3), discrepancies between RST and CDR greatly increased as the variance of the permeability field increased. The neglection of transverse dispersion by RST may have caused inaccurate results for highly nonuniform velocity 
fields. Another possibility could be that the CDR method was affected by numerical dispersion due to the highly nonuniform velocity fields. Further work is required to determine the cause of the discrepancies for these high variance cases.

For reactive transport simulations, in which large multidimensional CDR simulations are often computationally very expensive, we found that the RST method was more computationally efficient than CDR (e.g. $4 \mathrm{x}$ for ${ }^{237} \mathrm{~Np}$ transport in section 4.5.1). In section 5.5.2 RST was 14 times faster than CDR, whereas in section 5.5.3 RST was 4 times faster. Surprisingly, the ratio in cpu times between CDR and RST remained relatively constant whether we tested a reactive or nonreactive problem indicating that the efficiency of RST did not increase for the reactive transport simulations. We believe that for more complicated reactive transport systems, RST will become more efficient. Chemical heterogeneities did not seem to affect the accuracy of the RST approach for these test problems. However, it is conceivable that reactions could act to increase the discrepancy in travel times between adjacent streamlines, amplifying the effects of transverse dispersion causing RST to fail more readily than in a nonreactive transport problem.

Streamtube methods are quickly gaining popularity in the field of reactive transport. A topic of future research is to better quantify when RST fails due to the neglection of transverse dispersion. We have studied a few problems in which RST fails. An important question to answer is: whether the method consistently fails at high $\ln (\mathrm{k})$ variances? As part of this work, it would also be important to prove that RST does err on the conservative side.

Another subject of future research would be to develop a method to approximate the number of streamlines needed to accurately characterize a particular flow field before using RST. Without such an approximation, RST will have to be used with more streamlines than really necessary in order to get an accurate result. Otherwise, one would have to iterate to determine how many streamlines are necessary and this defeats the purpose of the method which is increased computational efficiency. After conducting many simulations, we gained experience with RST making it possible estimate the proper number of streamlines required. For some cases, we then 
performed a sensitivity analysis to determine the optimum number of streamlines to properly characterize the flow field. However this was not done for all cases, therefore some of the RST simulations presented use more than the optimum number of streamlines. An intriguing topic for future work would be the development of a technique to "add" streamlines to an existing solution instead of recalculating the full set of streamlines and one-dimensional simulations. In addition, for a given random field, it may be possible to use statistics, such as the variance in the permeability or velocity distribution and the correlation length, to determine the number of streamlines necessary to obtain an accurate solution. If such a method could be developed RST becomes much more powerful.

\subsection{The Unsaturated Zone Transport Test}

The UZTT results indicate that the models we have developed are capturing the major processes controlling nonreactive tracer transport through the Calico Hills. Two major questions answered by the test are that matrix flow is predominant in the Calico Hills and capillary forces are very strong. The actual measurements for all but two collection boreholes fall within the predicted $5 \%$ and $50 \%$ concentration breakthroughs. One collection borehole, which lies above the collection plane, matches the models well indicating that the model is correctly balancing the capillary and gravitational forces. Tracer approaches most of the collection boreholes below the injection plane first due to gravity, but eventually capillary action allows tracer to reach the one borehole above the plane. This complex phenomena is predicted by the model. The two breakthrough times that are slower than the actual measurements are very close to the injection plane. In fact, the accuracy of the model seems to increase as the distance between the injection and collection points increases. This may be an indication of short preferential pathways such as fractures that facilitate the transport of tracer to the boreholes that are close to the injection plane.

For the boreholes that are farther from the injection plane, any fracture flow may be sucked up by the matrix blocking preferential pathways. A topic of future work will be to determine whether the 
model predictions produce accurate results for the reactive tracers Lithium, Manganese and Cobalt which are radionuclide analogs. 


\section{REFERENCES}

Altman, S.J., B.W. Arnold, C.K. Ho, S.A. McKenna, R.W. Barnard, G.E. Barr, and R.E. Eaton, Flow calculations for Yucca Mountain ground water travel time (GWTT-95), Technical Report SAND96-0819, Sandia National Laboratories, 1996.

Batycky, R.P., Blunt, M.J., and Thiele, M.R., A streamline simulator to model field scale, threedimensional flow, Proc. of the 5th European Conference on the Mathematics of Oil Recovery, Leoben, Austria, Sept. 3-6, 1996.

Bear, J. and A. Verruijt, Modeling Groundwater Flow and Pollution, Reidel Publishing Co., 1987.

Behie, A., D. Collins, P.A. Forsyth, and P.H. Sammon, Fully coupled multiblock wells in oil simula tion, SPE J., 535-542, 1985.

Bethke, C.M. Geochemical Reaction Modeling, Oxford University Press, 1996.

Blunt, M.J., Liu, K., and Thiel, M.R., A generalized streamline method to predict reservoir flow, Petroleum Geosciences, 2, 259-269, 1996.

Bodvarsson, G.S., T.M. Bandurraga, and Y.S. Wu, editors, The Site-Scale Unsaturated Zone Model of Yucca Mountain, Nevada, for the Viability Assessment, LBNL-40376, Berkeley, California: Lawrence Berkeley National Laboratory, 1997.

Brusseau, M.L., R.E. Jessup, and P.S.C. Rao, Modeling the transport of solutes infiuenced by multi process nonequilibrium, Water Resour. Res., 25: 1971-1988, 1989.

Bussod, G.Y. et al., LA Testing Status Report: Busted Butte Unsaturated Zone Transport Test, Los Alamos National Laboratory YMP Milestone SPU85M4, 1998.

Cederberg, G.A., R.L. Street, and J.O. Leckie, A groundwater mass transport and equilibrium chem istry model for multicomponent systems, Water Resour. Res., 21:8, 1095-1104, 1985.

Chipera, S.J., K. Carter-Krogh, D.T. Vaniman, D.L. Bish, and J.W. Carey, Preliminary three-di mensional mineralogical model of Yucca Mountain, Nevada, Los Alamos National Laboratory YMP Milestone SP321AM4, 1997a. 
Chipera, S.J., D.T. Vaniman, D.L. Bish, and J.W. Carey, Mineralogic variation in drill holes USW NRG-6, NRG-7/7a, SD-7, SD-9, SD-12, and UZ\#14: New data from 1996-1997 analyses, Los Alamos National Laboratory YMP Milestone SP321BM4, 1997b.

Clement, T.P., A Modular Computer Code for Simulating Reactive Multispecies Transport in 3Dimensional Groundwater Systems, Pacific Northwest Laboratory Report, PNNL-SA-11720, 1997.

Efurd, D.W., W. Runde, J.C. Banar, F.R. Roensch, D.L. Clark, P.D. Palmer, C.D. Tait, Measured solubilities and speciation neptunium and plutonium in $\mathrm{J}-13$ groundwater, Los Alamos Nation al Laboratory, Yucca Mountain Site Characterization Project Milestone 3411, 1996.

Engesgaard, P. and K.L. Kipp, A geochemical model for redox-controlled movement of mineral fronts in ground-water flow systems: a case of nitrate removal by oxidation of pyrite, Water Resour. Res., 28:3308-3327, 1992.

Flint, L.E., Matrix properties hydrogeologic units at Yucca Mountain Nevada, U.S. Geological Survey Open File Report, MOL 19970324.0046, GS95030831, 1996.

Flint, L.E., Characterization of hydrogeologic units using matrix properties, Yucca Mountain, Neva da, USGS Water-Resources Invest. Rept. 97-4243, 64 pp. Denver, Colorado: U.S. Geological Survey. TIC Catalog \#236515, RIS Accession \#MOL, 19980429.0512, 1998.

Gelhar, L.W., Stochastic Subsurface Hydrology, Prentice-Hall, Inc., Englewood Cliffs, N.J., 1993.

Ginn, T.R., C.S. Simmons, and B.D. Wood, Stochastic-convective transport with nonlinear reaction: Biodegradation with microbial growth, Water Resources Res., 31(11), 2689-2700, 1995.

Goltz, M.N., and P.V. Roberts, Interpreting organic solute transport data from a field experiment using physical nonequilibrium models, J. of Contam. Hydrol., 1:77-94, 1986.

Hammond, G.E., Modification of the Finite Element Heat and Mass Transfer Code to model Multicomponent Precipitation-Dissolution Reactions, Masters Thesis, University of Illinois at Urbana-Champaign (in Preparation).

Hutzler, N.J., J.C. Crittenden, J.S. Gierke, and A.S. Johnson, Transport of organic compounds with saturated groundwater flow: experimental results, Water Resour. Res., 22:285-295, 1986. 
Javendal, I., C. Doughty, and C.F. Tsang, Groundwater Transport: Handbook of Mathematical Mod els, AGU, WR Monograph 10, 1984.

Jennings, A.A. and D.J. Kirkner, Instantaneous equilibrium approximation analysis, J. Hydraul. Div. Am. Soc. Civ. Eng., 110, 1700-1717, 1984.

Kinzelbach, W., W. Schafer, and J. Herzer, Numerical modeling of natural and enhanced denitrifica tion processes in aquifers, Water Resour. Res., 27:1123:1135, 1991.

Lichtner, P.C., Continuum formulation of multicomponent-multiphase reactive transport, Rev. in Mineralogy, Vol. 34, Chapter 1, 1-81, 1996.

Lichtner, P.C., FLOTRAN User's Manual, Version 1.0, Los Alamos National Laboratory document, 1999.

Liu, C.W. and T.N. Narasimhan, Redox-controlled multiple-species reactive chemical transport, 1, model development, Water Resour. Res., 25: 869-882, 1989.

Loeven, C. A summary and discussion of hydrologic data from the Calico Hills nonwelded hy drogeologic unit at Yucca Mountain, Nevada, Technical Report LA-12376-MS, Los Alamos National Laboratory, 1993.

Nicoud, R.M. and D. Schweich, Solute transport in porous media with solid-liquid mass transfer limitations: application to ion exchange, Water Resour. Res., 25:1071-1082, 1989.

Pinder, G. and W. Gray, Finite Element Simulations in Surface and Subsurface Hydrology, Academic Press, New York, 1977.

Pollock, D.W., Semianalytical computational of path lines for finite-difference models, Groundwa ter, 26, No. 6, 743-750, 1988.

Reeves, M., N.A. Baker, and J.O. Duguid, Review and selection of unsaturated flow models, Civil ian Radioactive Waste Management and Operating Contractor Report Number B000000001425-2200-00001, 1994.

Rittman, B.E., and J.M. VanBriesen, Microbial processes in reactive transport modeling, Rev. in Mineralogy, Vol. 34, Chapter 7, 311-332, 1996. 
Robinson, B.A., A.V. Wolfsberg, H.S. Viswanathan, C.W. Zyvoloski, G.A. Zyvoloski, H.J. Turin, Modeling of flow, radionuclide migration, and environmental isotope distributions at Yucca Mountain, Los Alamos National Laboratory, Yucca Mountain Project Milestone 3468, 1997.

Robinson, B.A., H.S. Viswanathan, A.J. Valocchi, Efficient numerical techniques for multicomponent groundwater transport based upon simultaneous solution of strongly coupled subsets of chemical components, Adv. in Water Resour., in press, 1998.

Rubin, J., Transport of reacting solutes in porous media: relation between mathematical nature of problem formulation and chemical nature of reaction, Water Resour. Res., 19:1231-1252, 1983.

Saad, Y., and M.H. Schultz, GMRES: A generalized minimum residual algorithm for solving non symmetric linear systems, SIAM J. Sci. and Stat. Comput., 7:3, 856-869, 1986.

Schafer-Perini, A. and J.L. Wilson, Efficient and accurate front tracking for two-dimensional groundwater flow models, Water Resour. Res., 27:1471-1485, 1991.

Simmons, C.S., T.R. Ginn, and B.D. Wood, Stochastic-convective transport with nonlinear reaction: Mathematical framework, Water Resources Res. 31(11), 2675-2688, 1995.

Smith, R.W., A.L. Schafer, A.F.B. Tompson, Theoretical relationships between reactivity and permeability for monominerallic porous media, in Proceedings, XIX Sympossium of the Scien tific Basis of Nuclear Waste Management, edited by W.M. Murphy and D.F. Knecht, Mater. Res. Symp. Proc., 412 (in press), 1996.

Steefel, C.I. and A.C. Lasaga, A coupled model for transport of multiple chemical species and kinet ic precipitation/dissolution reactions with application to reactive flow in single phase hydro thermal systems, American Journal of Science, 294, 529-592, 1994.

Steefel, C.I. and T.B. MacQuarrie, Approaches to Modeling Reactive Transport, Rev. in Mineralo gy, Vol. 34, Chapter 2, 83-125, 1996.

Suarez, D.L. and J. Simunek, Solute transport modeling user variably variably saturated water flow conditions, Rev. in Mineralogy, Vol. 24, Chapter 5, 229-264, 1996. 
Tebes-Stevens, C. and A.J. Valocchi, Numerical solution techniques for reaction parameter sensitiv ity coefficients in multicomponent subsurface transport models, Hydraulic Engineering Series No. 59, Department of Civil and Environmental Engineering, University of Mllinois, UIUC-ENG-98-2009, 1998.

Tebes-Stevens, C., A.J. Valocchi, J.M. VanBriesen, and B.E. Rittmann, Multicomponent transport with coupled geochemical and microbiological reactions: model description and example simulations, J. Hydro., 209:8-26, 1998.

Thiele, M.R., Blunt, M.J., and Orr, F.M., Modeling flow in heterogeneous media using streamtubes- II. Compositional Displacements, In Situ, 19, No. 4, 367-391, 1995.

Tompson, A.F.B, and L.W. Gelhar, Numerical simulation of solute transport in three-dimensional, randomly heterogeneous porous medium, Wat. Resour. Res., 26(10), 2541-2562, 1990.

Tompson, A.F.B., A.L. Schafer, R.W. Smith, Impacts of physical and chemical heterogeneity on co contaminant transport in a sandy porous medium, Wat. Resour. Res., 32(4), 801-818, 1996.

Triay, I.R., A. Meijer, J.L. Conca, K.S. Kung, R.S. Rundberg, and E.A. Streitelmeier, Summary and synthesis report on radionuclide retardation for the Yucca Mountain Site Characterization Proj ect, Los Alamos National Laboratory YMP Milestone 3784, 1996a.

Triay, I.R., C.R. Cotter, S.M. Kraus, M.H. Huddleston, S.J. Chipera, D.L. Bish, Radionuclide sorp tion in Yucca Mountain tuffs with J-13 well water: Neptunium, Uranium, and Plutonium, Los Alamos National Laboratory Yucca Mountain Site Characterization Project Milestone 3338, Technical Report LA-12956-MS, 1996b.

Valocchi, A.J., Validity of the local equilibrium assumption for modeling sorbing solute transport through homogeneous soils, Water Resour. Res., 21:808-820, 1985.

Valocchi, A.J. and Malmstead, M., Accuracy of operator splitting for advection-dispersion-reaction problems, Water Resour. Res., 28: 1471-1476, 1992.

van der Zee, S.F. Lens, and M. Lauer, Prediction of phosphate transport in small columns with an approximate sorption kinetics model, Water Resour. Res., 25:1353-1365, 1989. 
van Genuchten, M.T., A closed form equation for predicting hydraulic conductivity of unsaturated soils, Soil Sci. Soc. Am. J., 44,892-898, 1980.

Viswanathan, H.S., Modification of the finite element heat and mass transfer code (FEHMN) to model multicomponent reactive transport, M.S. Thesis, University of Illinois, Champaign-Ur bana, Illinois, Technical Report LA-13167-T, Los Alamos National Laboratory, 1996.

Viswanathan, H.S., B.A. Robinson, A.J. Valocchi, and I.R. Triay, A reactive transport model of nep tunium migration from the potential repository at Yucca Mountain, Journal of Hydrology, 1998.

Wilson, M.L. et al., Total-system performance assessment for Yucca Mountain-SNL second itera tion, Report SAND93-2675, Sandia National Laboratories, 1994.

Wolery, T.J., A computer program for geochemical aqueous speciation-solubility calculations: theoretical manual, user's guide, and related documentation (version 7.0). Technical Report UCRL-MA-1 10662-PT-IV, Lawrence Livermore National Laboratory.

Wood, W.W., T.F. Kraemer, and P.P. Hearn, Intragranular diffusion: an important mechanism in fluencing solute transport in clastic aquifers?, Science, 247: 1569-1572, 1990.

Yabusaki, S.B., C.I. Steefel, B.D. Wood, Multidimensional, multicomponent, subsurface reactive transport in nonuniform velocity fields: code verification using an advective reactive streamtube approach, Journal of Contaminant Hydrology (30)3-4, 1998.

Yeh, G.T. and V.S. Tripathi, A critical evaluation of recent developments in hydrogeochemical transport models of reactive multichemical components, Water Resour. Res., 25(1), 93-108, 1989.

Zyvoloski, G.A., Finite element methods for geothermal reservoir simulations, Int. J: Numer. Meth. Geomech., 7:75-86, 1983.

Zyvoloski, G.A., Incomplete factorization methods for finite elements, Int. J. Numer. Meth., Engng., 23:1101-1109, 1986.

Zyvoloski, G.A., B.A. Robinson, Z.V. Dash, L.L. Trease, Summary of models and methods for FEHM application -A Finite Element Heat and Mass Transfer Code, LA-13307-MS, 1997. 


\section{VITA}

Hari Selvi Viswanathan Upon completion of High School in 1989, Hari enrolled at the University of California at Santa Barbara, where he received a Bachelor's of Science degree in Chemical Engineering in 1993. Hari worked as an intern for Los Alamos National Laboratory during the summers from 1987-1998. Following his graduation, Hari accepted a research assistantship at the University of Illinois at Urbana-Champaign, where he obtained a Master's of Science degree in Environmental Engineering in Civil Engineering in 1995. In 1995 Hari began working toward a Doctor of Philosophy degree in Environmental Engineering. During his enrollment, Hari has continued to collaborate with Los Alamos National Laboratory on topics concerning his dissertation. Hari's graduate research has been published in several journals and reports. 